\title{
A MEASUREMENT OF TOP ANTI-TOP QUARK PAIR PRODUCTION CROSS SECTION IN PROTON ANTI-PROTON COLLISIONS AT A CENTER-OF-MASS ENERGY OF $1.96 \mathrm{TeV}$
}

\section{DISSERTATION}

Presented in Partial Fulfillment of the Requirements for the Degree Doctor of Philosophy in the Graduate School of The Ohio State University

$$
\text { By }
$$

Jongyoung Chung, B.S., M.S.

$$
* * * * *
$$

The Ohio State University

2003

Dissertation Committee:

Approved by

Prof. Brian L. Winer, Adviser

Prof. Richard E. Hughes

Prof. Eric Braaten

Adviser

Prof. Jonathan P. Pelz 
(c) Copyright by

Jongyoung Chung

2003 


\begin{abstract}
A measurement of the top anti-top quark pair production cross section in $p \bar{p}$ collisions at a center-of-mass energy of $1.96 \mathrm{TeV}$ is presented. The measurement is made using data with integrated luminosity of $109.4 \mathrm{pb}^{-1}$, obtained at the Collider Detector at Fermilab between August 2002 and May 2003. A search is made for the dilepton decay channel of top anti-top production, $t \bar{t} \rightarrow \ell^{+} \nu_{\ell} b \ell^{-} \overline{\nu_{\ell}} \bar{b}$. The Standard Model dilepton decay channel of the top anti-top quark pair production involves two high transverse momentum leptons, a large missing energy from the undetected neutrinos, two jets from the $b$ and $\bar{b}$ quark fragmentations. Various Standard Model processes can mimic $t \bar{t}$ production in this decay channel. These backgrounds are estimated to be $0.65 \pm 0.13$ events in an integrated luminosity of $109.4 p b^{-1}$. In the data, one $e e$, three $\mu \mu$ and two $e \mu$ dilepton candidates were observed. From these data, the $t \bar{t}$ production cross section of $\sigma_{t \bar{t}}=11.4_{-4.6}^{+6.2} p b$ has been measured. This measurement is consistent with the theoretical prediction of the Standard Model, $\sigma_{t \bar{t}}=6.70{ }_{-0.88}^{+0.71} p b$ assuming a top quark mass of $175 \mathrm{GeV} / \mathrm{c}^{2}$.
\end{abstract}




\section{ACKNOWLEDGMENTS}

I would like to thank my adviser Brian Winer for his invaluable support and guidance during my graduate work at Ohio State. I also thank Richard Hughes for his brilliant ideas and enthusiasm to help me. Both of them are great physicists. I learned a lot from them about carrying out researches and learned from their personalities.

I thank my colleagues, Phil Koehn, Evelyn Thomson, Chris Neu, Catalin Ciobanu, Carlos Sanchez, Radu Marginean. I thank Phil for guiding me in the dilepton working group. I thank Evelyn for providing analysis tools for my research. I thank Chris for making my life in the United States more enjoyable.

I thank Professor Eric Braaten for correcting errors in my thesis.

I thank my parents for their love. 


\title{
VITA
}

\author{
16 January 1973 ................. Born - Seoul, Korea \\ February $1995 \ldots \ldots \ldots \ldots \ldots \ldots \ldots \ldots \ldots$. . . in Physics, \\ Yonsei University, \\ Seoul, Korea \\ September 1997 - June 1999 ........... Graduate Teaching Assistant, \\ Yonsei University, \\ Seoul, Korea \\ August 1999 ....................... in Physics, \\ Yonsei University, \\ Seoul, Korea \\ September 1999 - August 2000 ......... Graduate Teaching Associate, \\ Ohio State University, \\ Columbus, $\mathrm{OH}$ \\ September 2000 - December 2003 ....... Graduate Research Associate, \\ Ohio State University, \\ Columbus, $\mathrm{OH}$
}

\section{PUBLICATIONS}

The CDF Collaboration, "Search For Associated Production Of Upsilon And Vector Boson In P Anti-P Collisions At $\mathrm{S}^{* *}(1 / 2)=1.8-$ Tev", Phys. Rev. Lett. $\underline{90}, 221803$ (2003).

The CDF Collaboration, "Central pseudorapidity gaps in events with a leading antiproton at the Fermilab Tevatron anti-p p collider", Phys. Rev. Lett. 91, 011802 (2003). 
The CDF Collaboration, "Search for the supersymmetric partner of the top quark in dilepton events from $\mathrm{p}$ anti-p collisions at $\mathrm{s}^{* *}(1 / 2)=1.8-\mathrm{TeV}^{\prime}$, Phys. Rev. Lett. $\underline{90}, 251801$ (2003).

The CDF Collaboration, "Cross section for forward J/ psi production in pp collisions at $\mathrm{s}=1.8 \mathrm{TeV}$ ", Phys. Rev. D. $\underline{66}, 092001$ (2002).

The CDF Collaboration, "Search for long-lived charged massive particles in anti-p p collisions at s**1/2 =1.8-TeV", Phys. Rev. Lett. 무, 131801 (2003).

The CDF Collaboration, "Search for a W' boson decaying to a top and bottom quark pair in 1.8-TeV p anti-p collisions", Phys. Rev. Lett. 므, 081802 (2003).

The CDF Collaboration, "Search for radiative b-hadron decays in $\mathrm{p}$ anti-p collisions at $\mathrm{s}^{* *}(1 / 2)=1.8-\mathrm{TeV} "$ Phys. Rev. D $\underline{66}, 112002$ (2002).

The CDF Collaboration, "Momentum distribution of charged particles in jets in dijet events in $\mathrm{p}$ anti-p collisions at $\mathrm{s}^{* *}(1 / 2)=1.8-\mathrm{TeV}$ and comparisons to perturbative QCD predictions", Phys. Rev. D $\underline{68}, 012003$ (2003).

The CDF Collaboration, "Measurement of the ratio of b quark production cross sections in anti-p p collisions at $\mathrm{s}^{* *}(1 / 2)=630-\mathrm{GeV}$ and $\mathrm{s}^{* *}(1 / 2)=1800-\mathrm{GeV}^{\prime}$, Phys. Rev. D $\underline{66}, 032002$ (2002).

The CDF Collaboration, "Branching ratio measurements of exclusive B+ decays to charmonium with the Collider Detector at Fermilab", Phys. Rev. D $\underline{66}, 052005$ (2002).

The CDF Collaboration, "Limits on extra dimensions and new particle production in the exclusive photon and missing energy signature in $\mathrm{p}$ anti-p collisions at $\mathrm{s}^{* *}(1 / 2)$ =1.8-TeV", Phys. Rev. Lett. $\underline{89}, 281801$ (2002).

The CDF Collaboration, "Measurement of B meson lifetimes using fully reconstructed B decays produced in $\mathrm{p}$ anti-p collisions at $\mathrm{s}^{* *}(1 / 2)=1.8-\mathrm{TeV}^{\prime}$, Phys. Rev. D 65, 092009 (2002).

The CDF Collaboration, "Search for new physics in photon lepton events in $\mathrm{p}$ anti-p collisions at s** $(1 / 2)=1.8-\mathrm{TeV} "$, Phys. Rev. Lett. $\underline{89}, 041802$ (2002). 
The CDF Collaboration, "Upsilon production and polarization in p anti-p collisions at $\mathrm{s}^{* *}(1 / 2)=1.8-\mathrm{TeV} "$, Phys. Rev. Lett. $\underline{88}, 161802$ (2002).

The CDF Collaboration, "Soft and hard interactions in p anti-p collisions at s**(1/2)

$=1800-\mathrm{GeV}$ and 630-GeV", Phys. Rev. D $\underline{65}, 072005$ (2002).

The CDF Collaboration, "Measurement of the B+ total cross section and B+ differential cross section dsigma/dp $(\mathrm{T})$ in $\mathrm{p}$ anti-p collisions at $\mathrm{s}^{* *}(1 / 2)=1.8-\mathrm{TeV}^{\prime}$, Phys. Rev. D $\underline{65}, 052005$ (2002).

The CDF Collaboration, "Search for single top quark production in p anti-p collisions at s**(1/2) =1.8-TeV", Phys. Rev. D $\underline{65}, 091102$ (2002).

E.J. Thomson et al., "Online track processor for the CDF upgrade", IEEE Trans. Nucl. Sci. 49, 1063 (2002).

\section{FIELDS OF STUDY}

Major Field: Physics 


\section{TABLE OF CONTENTS}

\section{Page}

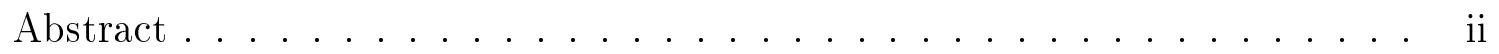

Acknowledgments .......................... ii

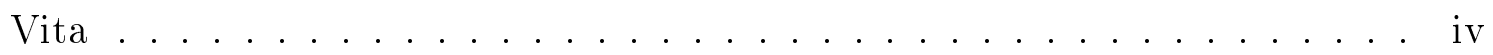

List of Tables ..........................

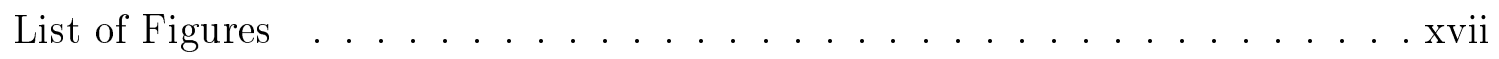

Chapters:

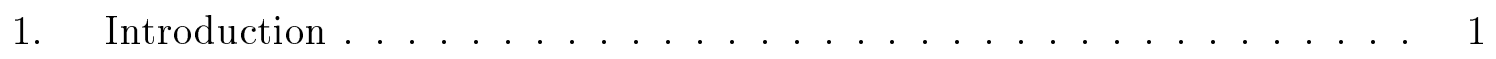

1.1 Overview of the Standard Model . . . . . . . . . . . . . 1

1.2 Top Quark Physics . . . . . . . . . . . . . . . . . 3

1.3 Top Quark Production Mechanism . . . . . . . . . . . 5

1.4 Top Quark Decay . . . . . . . . . . . . . . . . . . 8

1.5 Dilepton Decay Channel . . . . . . . . . . . . . . . 10

1.6 Overview of thesis . . . . . . . . . . . . . . 12

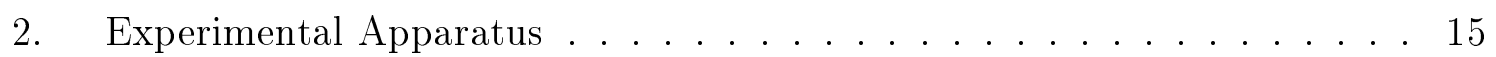

2.1 The Tevatron Collider . . . . . . . . . . . . . . 15

2.2 The CDF Detector . . . . . . . . . . . . . 18

2.2.1 The Tracking System . . . . . . . . . . . . 20

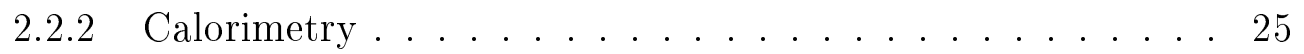

2.2.3 The Muon Detectors . . . . . . . . . . . . . 30

2.2.4 Event Triggers .................. 34 
3. Event Selection and Acceptance of $t \bar{t}$ event . . . . . . . . . . . . 36

3.1 Electron Selection . . . . . . . . . . . . . . . 40

3.1.1 Electron Identification Requirements . . . . . . . . . . 41

3.2 Muon Selection . . . . . . . . . . . . . . . . . . 43

3.2.1 Muon Identification Requirements _. . . . . . . . . . . 45

3.3 Dilepton Selection . . . . . . . . . . . . . . 4 47

3.3 .1 Isolation . . . . . . . . . . . . . . . . . 47

3.3 .2 Invariant Mass . . . . . . . . . . . . . . . . . . 49

3.3 .3 Missing $E_{T} \ldots \ldots \ldots \ldots \ldots \ldots \ldots \ldots$

3.3 .4 Jet Requirements . . . . . . . . . . . . . . . . . . 54

3.3.5 $\quad H_{T}$ and Opposite Sign Requirement $\ldots \ldots \ldots \ldots 59$

3.3.6 Considerations for cross section measurement . . . . . . . 63

3.4 Dilepton Channel Acceptance . . . . . . . . . . . . . . . . . 64

3.4.1 Monte Carlo Samples . . . . . . . . . . . . . . . 65

3.4.2 Acceptance and Kinematic Properties of $t \bar{t}$ events . . . . . . 65

3.4.3 Lepton Identification Efficiency . . . . . . . . . . . . . 66

3.4.4 Systematic Uncertainty . . . . . . . . . . . . . 72

4. Backgrounds in Dilepton events . . . . . . . . . . . 78

4.1 Drell-Yan process . . . . . . . . . . . . . . 78

$4.2 \quad Z^{0} \rightarrow \tau^{+} \tau^{-} \ldots \ldots \ldots \ldots \ldots \ldots \ldots \ldots \ldots$

4.3 Vector boson pair production . . . . . . . . . . . 92

4.4 Fake leptons . . . . . . . . . . . . . . . . . 94

4.5 Summary of the Backgrounds . . . . . . . . . . . 107

5. Results . . . . . . . . . . . . . . . . . . . . . 109

5.1 Data sample . . . . . . . . . . . . . . . . . . 109

5.2 The $t \bar{t}$ dilepton candidate events $\ldots \ldots \ldots \ldots \ldots$

5.3 The $t \bar{t}$ cross section . . . . . . . . . . . . . . 115

6. Conclusion . . . . . . . . . . . . . . . . . . . . 120

Appendices:

A. Lepton Identification Efficiencies . . . . . . . . . . . . . . 121 
B. Selection of the Optimal Scenario . . . . . . . . . . . . . . 139

C. Event Displays of the Dilepton Candidates . . . . . . . . . . . 149

Bibliography . . . . . . . . . . . . . . . . . . 162 


\section{LIST OF TABLES}

Table

Page

1.1 Quark and lepton $\mathrm{SU}_{L}(2)$ multiplets ............. 2

1.2 The $t \bar{t}$ production cross section. CDF [18], D $\varnothing$ [21], and theoretical prediction [16]. The top quark masses $m_{t}$ in the table are used for the calculations of the cross section. . . . . . . . . . 7

$1.3 t \bar{t}$ decay modes and their branching ratios. . . . . . . . . . . . 10

1.4 Cross section measurements using different $t \bar{t}$ decay channels in $p \bar{p}$ collisions at $\sqrt{s}=1.8 \mathrm{TeV}$ from the CDF collaboration $\left(m_{t}=\right.$

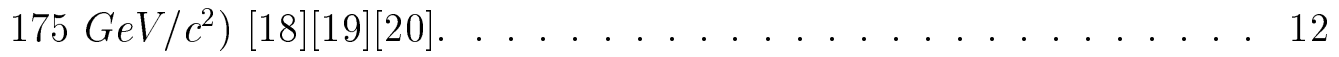

2.1 Description of the Central Outer Tracker (COT). . . . . . . . 23

2.2 Comparison of SVX and SVX II. . . . . . . . . . . . . 24

2.3 The physical properties for the central and endwall calorimeters. In the last row, the symbol $\oplus$ indicates that the constant term is to be added in quadrature to the resolution. . . . . . . . . . 30

2.4 Characteristics of the plug calorimeters. . . . . . . . . . 32

3.1 Selection cuts for TCE identification variables. . . . . . . . . . . 44

3.2 Selection cuts for PEM and PHX identification variables. . . . . . . . 44

3.3 Selection cuts for the central muon and minimum ionizing particle identification variables. . . . . . . . . . . . . 46 
3.4 Dilepton categories for $e e, \mu \mu, e \mu \ldots \ldots \ldots \ldots$

3.5 Dilepton categories for $e e, \mu \mu, e \mu$ without PHX, PEM and CMIO. . . 64

3.6 The relative acceptances to the $t \bar{t}$ dilepton signal (for the $e e, \mu \mu, e \mu$ separately) from various decay channels $(W W \rightarrow e e, \mu \mu, \ldots)$. The first six rows show the relative acceptances when $W W$ decays to two leptons at the generator level. The next three rows correspond to one $W$ decaying to a lepton, the other decaying hadronically. In the next three rows, $(b \rightarrow \ell)$ means that a $b$-quark decays semileptonically. The last row corresponds to the all hadronic decays of the $W W$. All the numbers are counted after only the lepton identification requirements. Errors are statistical only. . . . . . . . . . . . 68

3.7 The relative acceptances to the $t \bar{t}$ dilepton signal (for the $e e, \mu \mu, e \mu$ separately) from various decay channels $(W W \rightarrow e e, \mu \mu, \ldots)$. The first six rows show the relative acceptances when $W W$ decays to two leptons at the generator level. The next three rows correspond to one $W$ decaying to a lepton, the other decaying hadronically. In the next three rows, $(b \rightarrow \ell)$ means that a $b$-quark decays semileptonically. The last row corresponds to the all hadronic decays of the $W W$. All the numbers are counted after only the lepton identification requirements and the isolation requirement. Errors are statistical only. . . . 69

3.8 Results from the dilepton selection on the $t \bar{t}$ PYTHIA sample $\left(m_{t}=\right.$ $175 \mathrm{GeV} / \mathrm{c}^{2}, 366,366$ events). . . . . . . . . . . . . 70

3.9 The cut efficiencies after the lepton identification on the $t \bar{t}$ PYTHIA sample $\left(m_{t}=175 \mathrm{GeV} / \mathrm{c}^{2}, 366,366\right.$ events). The efficiencies are calculated from the events that passed all preceding dilepton selections. All errors are statistical only. . . . . . . . . . . . . . 71

3.10 Result from the dilepton selection on the $t \bar{t}$ PYTHIA sample $\left(m_{t}=\right.$ $175 \mathrm{GeV} / \mathrm{c}^{2}, 366,366$ events). The numbers in the last column are obtained by multiplying the weighting factor to the numbers of events passing all dilepton selections in the second column. . . . . . . . 73 
3.11 The acceptance of fiducial, $p_{T}$ and lepton identification $\left(\epsilon_{I D}\right)$ and the cut efficiencies after the lepton identification requirements. The final dilepton acceptance $\left(\epsilon_{d i l}\right)$ is the probability that a $t \bar{t}$ event passes all the dilepton selection requirements. The number in the last row is the dilepton acceptance with the identification efficiency correction. All errors are statistical only. . . . . . . . . . . . . . . . . 74

3.12 Contributions from various sources of systematic uncertainty to the dilepton acceptance. . . . . . . . . . . . . 77

4.1 The numbers of events before the $H_{T}$ cut and after the $H_{T}$ cut using the PYTHIA Drell-Yan Monte Carlo sample $\left(2.3 \mathrm{fb}^{-1}\right.$ for the electron channel, $2.6 \mathrm{fb}^{-1}$ for the muon channel). $\epsilon_{H_{T}}$ is the $H_{T}$ cut efficiency with $H_{T}>200 \mathrm{GeV}$. . . . . . . . . . . . . . . . . . . . . . . . 84

4.2 The numbers of events used for the Drell-Yan background calculation and the result in $109 p b^{-1}$ of data. $\left\langle m_{\ell \ell}\right\rangle$ is the mean dilepton invariant mass. The $N_{Z^{0}}^{\text {pass }}$ is the number of events inside the $Z^{0}$ mass peak passing all the dilepton cuts. The $N_{Z^{0}}^{t \bar{t}}$ is the number of the $t \bar{t}$ events estimated with the PYTHIA $t \bar{t}$ Monte Carlo sample inside the $Z^{0}$ mass peak and passing all the dilepton cuts. The $N_{b q}^{D Y}$ is the estimated background from the Drell-Yan process in $109 p b^{-1}$ of data. 84

4.3 Result from the dilepton selection on the PYTHIA $Z^{0} \rightarrow \tau^{+} \tau^{-}$sample $(416,499$ events). . . . . . . . . . . . . . . . . . . 90

4.4 Result from the dilepton selection on the $W W$ PYTHIA sample $(827,323$ events). . . . . . . . . . . . . . 95

4.5 The requirements of the control sample 1 and control sample 2 for the fake ratio calculation. . . . . . . . . . . . . . . . . . 104

4.6 The result of counting the control sample 1 and control sample 2 from the jet 20 sample. . . . . . . . . . . . . . . . 105

4.7 The result of counting the $W+\geq 3$ jet events with one lepton of control sample 2 type before the $H_{T}$ and the opposite sign requirement applied in the data. The fake ratio of each lepton type is applied to these numbers to give the expected backgrounds from the fake lepton in $109 p b^{-1}$. . . . . . . . . . . . . . . . . 105 
4.8 The result of counting the $W+\geq 3$ jet events with one lepton of control sample 2 type after the $H_{T}$ and before the opposite sign requirement applied in the data. The fake ratio of each lepton type is applied to these numbers to give the expected backgrounds from the

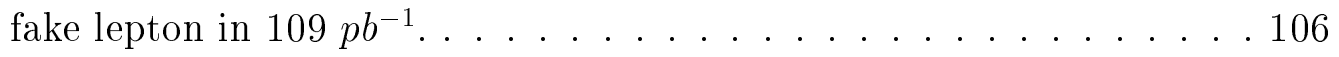

4.9 The result of counting the $W+\geq 3$ jet events with one lepton of control sample 2 type after all the dilepton selection requirements applied in the data. The fake ratio of each lepton type is applied to these numbers to give the expected backgrounds from the fake lepton in $109 p b^{-1}$. . . . . . . . . . . . . . . 106

4.10 The background contributions to the dilepton channels in $109 p b^{-1}$ of integrated luminosity. The contributions from 0 jet and 1 jet bin are separately shown before the $H_{T}$ is applied. The expected number of events from $t \bar{t}$ production using a PYTHIA Monte Carlo sample with $m_{t}=175 \mathrm{GeV} / \mathrm{c}^{2}$ are also shown. . . . . . . . . . . . . 108

5.1 The selection cuts for the electrons in the inclusive electron sample. At least one electron passing these cuts is required for the event to be included in the inclusive electron sample. This sample is used for the dilepton analysis. . . . . . . . . . . . . . . 110

5.2 The selection cuts for the muons in the inclusive muon sample. At least one muon passing these cuts is required for the event to be part of the inclusive muon sample. This sample is used for the dilepton analysis. . . . . . . . . . . . . . . . . 111

5.3 The result of applying the dilepton selection cuts on $109 p b^{-1}$ data. . 113

5.4 The dilepton $t \bar{t}$ candidates and their kinematic properties. $E_{T}(e)$ is the transverse energy when the lepton is an electron and $p_{T}(\mu)$ is the beam constrained transverse momentum when the lepton is a muon. The $E_{T}$ is the missing transverse energy corrected for muons and jets as described in section 3.3.3. All energies and momenta are in units of $\mathrm{GeV}$ and $\mathrm{GeV} / \mathrm{c}$. All quantities of jets are corrected using the techniques described in Section 3.3.4. . . . . . . . . . . . . 114 
A.1 The efficiency of the tight central electron (TCE) identification cuts using the $Z^{0} \rightarrow e^{+} e^{-}$data sample. All uncertainties are statistical. . . 123

A.2 The efficiency of the plug electron (PEM) identification cuts using the $Z^{0} \rightarrow e^{+} e^{-}$data sample. All uncertainties are statistical. . . . . . 124

A.3 The efficiencies of the Phoenix electron (PHX) identification cuts using the $Z^{0} \rightarrow e^{+} e^{-}$data sample. All uncertainties are statistical. The Phoenix Track matching cut is the efficiency that a PEM has a Phoenix track. The Phoenix Silicon hits, and Phoenix Track $z_{0}$ is obtained from the PEM that has a Phoenix track. The total efficiency is the probability that a PEM passes all PHX identification cuts and has a Phoenix track. . . . . . . . . . . . . . . 124

A.4 The efficiency of the central muon (CMUP) identification cuts using the $Z^{0} \rightarrow \mu^{+} \mu^{-}$data sample. All uncertainties are statistical. . . . 125

A.5 The efficiency of the central muon (CMU only) identification cuts using the $Z^{0} \rightarrow \mu^{+} \mu^{-}$data sample. All uncertainties are statistical. . 125

A.6 The efficiency of the central muon (CMP only) identification cuts using the $Z^{0} \rightarrow \mu^{+} \mu^{-}$data sample. All uncertainties are statistical. . 126

A.7 The efficiency of the central muon (CMX) identification cuts using the $Z^{0} \rightarrow \mu^{+} \mu^{-}$data sample. All uncertainties are statistical. . . . 126

A.8 The efficiency of the minimum ionizing particle (CMIO) identification cuts using the $Z^{0} \rightarrow \mu^{+} \mu^{-}$data sample. All uncertainties are statistical.127

A.9 The efficiency of the tight central electron (TCE) identification cuts using a $Z^{0} \rightarrow e^{+} e^{-}$PYTHIA sample. All uncertainties are statistical. 127

A.10 The efficiency of the plug electron (PEM) identification cuts using a $Z^{0} \rightarrow e^{+} e^{-}$PYTHIA sample. All uncertainties are statistical. . . . 128 
A.11 The efficiencies of the Phoenix electron (PHX) identification cuts using a $Z^{0} \rightarrow e^{+} e^{-}$PYTHIA sample. All uncertainties are statistical. The Phoenix Track matching cut is the efficiency that a PEM has a Phoenix track. The Phoenix Silicon hits, and Phoenix Track $z_{0}$ is obtained from the PEM that has a Phoenix track. The total efficiency is the probability that a PEM passes all PHX identification cuts and has a Phoenix track. . . . . . . . . . . . . . 128

A.12 The efficiency of the central muon (CMUP) identification cuts using a $Z^{0} \rightarrow \mu^{+} \mu^{-}$PYTHIA sample. All uncertainties are statistical. . . . 129

A.13 The efficiency of the central muon (CMU only) identification cuts using a $Z^{0} \rightarrow \mu^{+} \mu^{-}$PYTHIA sample. All uncertainties are statistical. 129

A.14 The efficiency of the central muon (CMP only) identification cuts using a $Z^{0} \rightarrow \mu^{+} \mu^{-}$PYTHIA sample. All uncertainties are statistical. 130

A.15 The efficiency of the central muon (CMX) identification cuts using a $Z^{0} \rightarrow \mu^{+} \mu^{-}$PYTHIA sample. All uncertainties are statistical. . . . . 130

A.16 The efficiency of the minimum ionizing particle (CMIO) identification cuts using a $Z^{0} \rightarrow \mu^{+} \mu^{-}$PYTHIA sample. All uncertainties are

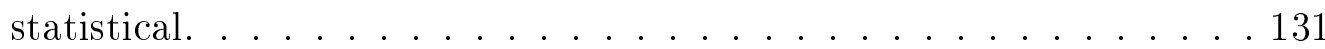

A.17 The ID efficiencies of leptons in data and a PYTHIA Monte Carlo sample. The scale factor is obtained from the efficiencies in the data divided by efficiencies in the Monte Carlo sample in each type of leptons. 131

B.1 Three scenarios of dilepton selections for the study of a priori cross section uncertainty. . . . . . . . . . . . . . . . . 143

B.2 Three scenarios of dilepton selections for the study of a priori cross section uncertainty. . . . . . . . . . . . . . 144

B.3 The result of the pseudoexperiments (100,000 events) in $200 \mathrm{pb}^{-1}$. The RMS values of the cross section distribution of the pseudoexperiments $\left(\delta \sigma_{t \bar{t}}^{p e}\right)$ represent the a priori uncertainties of the cross section measurements. . . . . . . . . . . . . . . . . . 144 
B.4 The result of the pseudoexperiments (100,000 events) in $500 p b^{-1}$. The RMS values of the cross section distribution of the pseudoexperiments $\left(\delta \sigma_{t \bar{t}}^{p e}\right)$ represent the a priori uncertainties of the cross section measurements. . . . . . . . . . . . . . . 145

B.5 The result of the pseudoexperiments (100,000 events) in $2 \mathrm{fb}^{-1}$. The RMS values of the cross section distribution of the pseudoexperiments $\left(\delta \sigma_{t \bar{t}}^{p e}\right)$ represent the a priori uncertainties of the cross section measurements. . . . . . . . . . . . . . . 145 


\section{LIST OF FIGURES}

Figure

Page

1.1 Lowest order Feynman diagrams for $t \bar{t}$ production via strong interaction in $p \bar{p}$ collisions. . . . . . . . . . . . . 6

$1.2 q \bar{q} \rightarrow t \bar{t}$ production and Standard Model $t \bar{t}$ decay. . . . . . . . . . 11

2.1 The instantaneous luminosity (top) and integrated luminosity (bottom) of the Tevatron since July 2001. . . . . . . . . . . . . . 17

2.2 An elevation view of one half of the CDF II detector. . . . . . . . . 19

2.3 The arrangement of drift cells at the east endplate of the COT. Only $1 / 6$ portion in $\phi$ is shown. . . . . . . . . . . 22

2.4 A schematic view of CDF run II detector showing the $\eta$ coverage of the tracking systems surrounded by the solenoid and the plug calorimeters. 26

2.5 An end view of the CDF silicon system including the SVX II bulkheads and ISL support structure. . . . . . . . . . . . 27

2.6 Diagram of a single central calorimetry wedge. . . . . . . . . . . 29

2.7 Geometry of a central calorimeter wedge and its towers. . . . . . . . 31

2.8 A transverse view of a muon chamber tower showing the offset of sense wire in the outer two layers. . . . . . . . . . . . 33

2.9 The coverage of CDF muon detectors in azimuth $\phi$ and pseudorapid-

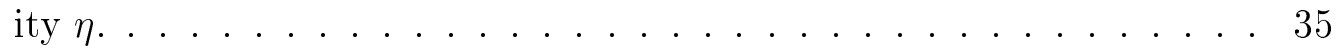


3.1 Electron $E_{T}$ of generator level (histogram) and reconstructed level (points) for the PYTHIA $t \bar{t}$ Monte Carlo sample with $m_{t}=175 \mathrm{GeV} / \mathrm{c}^{2}$. $W \rightarrow e \nu_{e}$ (top left), $W \rightarrow \tau \nu_{\tau} \rightarrow e \nu_{e} \nu_{\tau}$ (top right), $b \rightarrow e \nu_{e} c$ (bottom

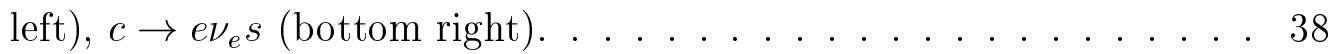

3.2 Muon $E_{T}$ of generator level (histogram) and reconstructed level (points) for the PYTHIA $t \bar{t}$ Monte Carlo sample with $m_{t}=175 \mathrm{GeV} / \mathrm{c}^{2}$. $W \rightarrow \mu \nu_{\mu}$ (top left), $W \rightarrow \tau \nu_{\tau} \rightarrow \mu \nu_{\mu} \nu_{\tau}$ (top right), $b \rightarrow \mu \nu_{\mu} c$ (bottom left), $c \rightarrow \mu \nu_{\mu} s$ (bottom right). . . . . . . . 39

3.3 Isolation of electrons from different sources in the $t \bar{t}$ PYTHIA Monte Carlo sample with $m_{t}=175 \mathrm{GeV} / \mathrm{c}^{2} . W \rightarrow e \nu_{e}$ (top left), $b \rightarrow e \nu_{e} c$ (top right), $W \rightarrow \tau \nu_{\tau} \rightarrow e \nu_{e} \nu_{\tau}$ (bottom left). Note scale change of

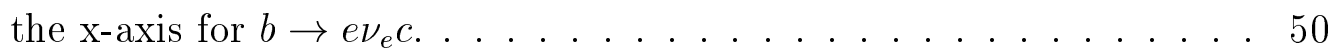

3.4 Isolation of muons from different sources in the $t \bar{t}$ PYTHIA Monte Carlo sample with $m_{t}=175 \mathrm{GeV} / \mathrm{c}^{2}$. $W \rightarrow \mu \nu_{\mu}$ (top left), $b \rightarrow \mu_{\mu} c$ (top right), $W \rightarrow \tau \nu_{\tau} \rightarrow \mu \nu_{\mu} \nu_{\tau}$ (bottom left). Note scale change of the x-axis for $b \rightarrow \mu \nu_{\mu} c . \ldots \ldots \ldots$. . . . . . . . . 51

3.5 Invariant mass of electron pairs (top) and muon pairs (bottom) in the dilepton events in $109 p b^{-1}$ of data. . . . . . . . . . . 52

3.6 Invariant mass of the two leptons $\left(m_{\ell \ell}\right)$ in the dilepton events after the isolation requirement is imposed. Di-electron (ee, top), di-muon ( $\mu \mu$, middle), electron-muon ( $e \mu$, bottom) events in the $t \bar{t}$ PYTHIA Monte Carlo sample with $m_{t}=175 \mathrm{GeV} / \mathrm{c}^{2}$. In the $e \mu$ category, the invariant mass cut is not imposed. The vertical lines in the $e e, \mu \mu$ show the invariant mass cut of $76 \mathrm{GeV} / \mathrm{c}^{2}<m_{\ell \ell}<106 \mathrm{GeV} / \mathrm{c}^{2}$. . . . 53

3.7 The magnitude of the missing $E_{T}\left(\mathbb{E}_{T}, x\right.$ axis) and the $\Delta \phi$ between $\mathbb{E}_{T}$ and the closest jet or lepton ( $y$ axis). Di-electron events in the $t \bar{t}$ PYTHIA Monte Carlo sample with $m_{t}=175 \mathrm{GeV} / \mathrm{c}^{2}$ are shown with no jet $\left(N_{j}=0\right.$, top left), $N_{j}=1$ (top right), $N_{j} \geq 2$ (bottom left), $N_{j} \geq 0$ (bottom right). The lines show the cut imposed for the

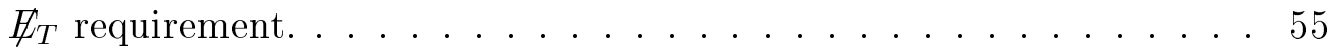


3.8 The magnitude of the missing $E_{T}\left(E_{T}, x\right.$ axis $)$ and the $\Delta \phi$ between $E_{T}$ and the closest jet or lepton (y axis). Di-muon events in the $t \bar{t}$ PYTHIA Monte Carlo sample with $m_{t}=175 \mathrm{GeV} / \mathrm{c}^{2}$ are shown with no jet $\left(N_{j}=0\right.$, top left), $N_{j}=1$ (top right), $N_{j} \geq 2$ (bottom left), $N_{j} \geq 0$ (bottom right). The lines show the cut imposed for the $\mathbb{E}_{T}$

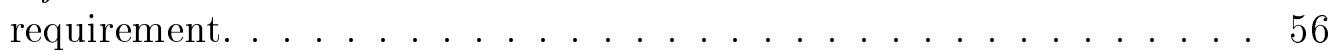

3.9 The magnitude of the missing $E_{T}\left(E_{T}, x\right.$ axis) and the $\Delta \phi$ between $E_{T}$ and the closest jet or lepton ( $y$ axis). $e \mu$ events in the $t \bar{t}$ PYTHIA Monte Carlo sample with $m_{t}=175 \mathrm{GeV} / \mathrm{c}^{2}$ are shown with no jet ( $N_{j}=0$, top left), $N_{j}=1$ (top right), $N_{j} \geq 2$ (bottom left), $N_{j} \geq 0$ (bottom right). The lines show the cut imposed for the $\not_{T}$ requirement. 57

3.10 The $H_{T}$ of di-electron events in the $t \bar{t}$ PYTHIA Monte Carlo sample with $m_{t}=175 \mathrm{GeV} / \mathrm{c}^{2}$ after $\mathbb{E}_{T}$ requirement. The plots are shown with no jet $\left(N_{j}=0\right.$, top left), $N_{j}=1$ (top right), $N_{j} \geq 2$ (bottom left), $N_{j} \geq 0$ (bottom right). The line shows the the cut for dilepton events $\left(H_{T}>200 \mathrm{GeV}\right)$. Only the events with $N_{j} \geq 2$ are selected for the final $t \bar{t}$ dilepton events. . . . . . . . . . . . . . 60

3.11 The $H_{T}$ of di-muon events in the $t \bar{t}$ PYTHIA Monte Carlo sample with $m_{t}=175 \mathrm{GeV} / \mathrm{c}^{2}$ after $\mathbb{E}_{T}$ requirement. The plots are shown with no jet $\left(N_{j}=0\right.$, top left), $N_{j}=1$ (top right), $N_{j} \geq 2$ (bottom left), $N_{j} \geq 0$ (bottom right). The line shows the the cut for dilepton events $\left(H_{T}>200 \mathrm{GeV}\right)$. Only the events with $N_{j} \geq 2$ are selected for the final $t \bar{t}$ dilepton events. . . . . . . . . . . . . . 61

3.12 The $H_{T}$ of $e \mu$ events in the $t \bar{t}$ PYTHIA Monte Carlo sample with $m_{t}=175 \mathrm{GeV} / \mathrm{c}^{2}$ after $\mathbb{E}_{T}$ requirement. The plots are shown with no jet $\left(N_{j}=0\right.$, top left), $N_{j}=1$ (top right), $N_{j} \geq 2$ (bottom left), $N_{j} \geq 0$ (bottom right). The line shows the the cut for dilepton events $\left(H_{T}>200 \mathrm{GeV}\right)$. Only the events with $N_{j} \geq 2$ are selected for the final $t \bar{t}$ dilepton events. . . . . . . . . . . . . . . . 62

3.13 The $p_{T}$ and $\eta$ distributions of the leptons from the $W$ decay at the generator level of the $t \bar{t}$ PYTHIA Monte Carlo sample with $m_{t}=$ $175 \mathrm{GeV} / \mathrm{c}^{2}$. Electron $p_{T}$ (top left), $\eta$ (top right), muon $p_{T}$ (bottom left), $\eta$ (bottom right). . . . . . . . . . . . . 67

4.1 Drell-Yan process. . . . . . . . . . . . . . 79 
4.2 Azimuthal angle between the $\not_{T}$ and the closest lepton or jet, versus the $\mathbb{E}_{T}$ for $Z^{0} \rightarrow e^{+} e^{-}$data $\left(109 p b^{-1}\right) . \quad \ldots \ldots \ldots \ldots$. . . . 81

4.3 Azimuthal angle between the $\not_{T}$ and the closest lepton or jet, versus the $\not_{T}$ for $Z^{0} \rightarrow \mu^{+} \mu^{-}$data $\left(109 p b^{-1}\right) . \ldots \ldots \ldots 2$

4.4 The magnitude of the missing $E_{T}\left(\not_{T}, x\right.$ axis) and the $\Delta \phi$ between $E_{T}$ and the closest jet or lepton ( $y$ axis). Di-electron events in the $Z^{0} \rightarrow$ $\tau^{+} \tau^{-}$PYTHIA Monte Carlo sample are shown with no jet $\left(N_{j}=0\right.$, top left), $N_{j}=1$ (top right), $N_{j} \geq 2$ (bottom left), $N_{j} \geq 0$ (bottom right). The lines show the cut imposed for the $\mathbb{E}_{T}$ requirement. . . 86

4.5 The magnitude of the missing $E_{T}$ ( $\mathbb{E}_{T}, x$ axis) and the $\Delta \phi$ between $E_{T}$ and the closest jet or lepton ( $y$ axis). Di-muon events in the $Z^{0} \rightarrow$ $\tau^{+} \tau^{-}$PYTHIA Monte Carlo sample are shown with no jet $\left(N_{j}=0\right.$, top left), $N_{j}=1$ (top right), $N_{j} \geq 2$ (bottom left), $N_{j} \geq 0$ (bottom right). The lines show the cut imposed for the $\mathbb{E}_{T}$ requirement. . . .

4.6 The magnitude of the missing $E_{T}\left(\mathbb{E}_{T}, x\right.$ axis) and the $\Delta \phi$ between $E_{T}$ and the closest jet or lepton ( $y$ axis). $e \mu$ events in the $Z^{0} \rightarrow \tau^{+} \tau^{-}$ PYTHIA Monte Carlo sample are shown with no jet $\left(N_{j}=0\right.$, top left), $N_{j}=1$ (top right), $N_{j} \geq 2$ (bottom left), $N_{j} \geq 0$ (bottom right). The lines show the cut imposed for the $\mathbb{E}_{T}$ requirement. . . 88

4.7 Invariant mass of two leptons $\left(m_{\ell \ell}\right)$ in the dilepton events of the PYTHIA $Z^{0} \rightarrow \tau^{+} \tau^{-}$Monte Carlo sample after the isolation requirement is imposed. Di-electron (ee, top), di-muon ( $\mu \mu$, middle), electron-muon ( $e \mu$, bottom) events are shown separately. In the $e \mu$ category, the invariant mass cut is not imposed. The red lines in the $e e, e \mu$ show the invariant mass cut of $76 \mathrm{GeV} / \mathrm{c}^{2}<m_{\ell \ell}<106 \mathrm{GeV} / \mathrm{c}^{2}$.

4.8 Diagrams for the tree level $W W$ production. . . . . . . . . . . 93

4.9 The magnitude of the missing $E_{T}$ ( $\mathscr{E}_{T}, x$ axis) and the $\Delta \phi$ between $E_{T}$ and the closest jet or lepton ( $y$ axis). Di-electron events in the $W W$ PYTHIA Monte Carlo sample are shown with no jet $\left(N_{j}=0\right.$, top left), $N_{j}=1$ (top right), $N_{j} \geq 2$ (bottom left), $N_{j} \geq 0$ (bottom right). The lines show the cut imposed for the $\not_{T}$ requirement. This sample is equivalent to $608.5 \mathrm{fb}^{-1} . \ldots \ldots \ldots \ldots$ 
4.10 The magnitude of the missing $E_{T}$ ( $\mathbb{E}_{T}, x$ axis) and the $\Delta \phi$ between $E_{T}$ and the closest jet or lepton ( $y$ axis). Di-muon events in the $W W$ PYTHIA Monte Carlo sample are shown with no jet $\left(N_{j}=0\right.$, top left), $N_{j}=1$ (top right), $N_{j} \geq 2$ (bottom left), $N_{j} \geq 0$ (bottom right). The lines show the cut imposed for the $\not_{T}$ requirement. This sample is equivalent to $608.5 \mathrm{fb}^{-1} \ldots \ldots \ldots \ldots$. . . . . 97

4.11 The magnitude of the missing $E_{T}$ ( $\mathbb{E}_{T}, x$ axis) and the $\Delta \phi$ between $E_{T}$ and the closest jet or lepton ( $y$ axis). $e \mu$ events in the $W W$ PYTHIA Monte Carlo sample are shown with no jet $\left(N_{j}=0\right.$, top left), $N_{j}=1$ (top right), $N_{j} \geq 2$ (bottom left), $N_{j} \geq 0$ (bottom right). The lines show the cut imposed for the $\not_{T}$ requirement. This sample is equivalent to $608.5 \mathrm{fb}^{-1} \ldots \ldots \ldots \ldots \ldots$

4.12 Invariant mass of two leptons $\left(m_{\ell \ell}\right)$ in the dilepton events of the PYTHIA $W W$ Monte Carlo sample after the isolation requirement imposed. Di-electron (ee, top), di-muon ( $\mu \mu$, middle), electron-muon ( $e \mu$, bottom) events are shown separately. In the $e \mu$ category, the invariant mass cut is not imposed. The red lines in the $e e, e \mu$ show the invariant mass cut of $76 \mathrm{GeV} / \mathrm{c}^{2}<m_{\ell \ell}<106 \mathrm{GeV} / \mathrm{c}^{2}$. This sample is equivalent to $608.5 \mathrm{fb}^{-1}$. . . . . . . . . . . 99

4.13 The $H_{T}$ of di-electron events after $E_{T}$ requirement in the $W W$ PYTHIA Monte Carlo sample, equivalent to $608.5 \mathrm{fb}^{-1}$. The plots are shown with no jet $\left(N_{j}=0\right.$, top left), $N_{j}=1$ (top right), $N_{j} \geq 2$ (bottom left), $N_{j} \geq 0$ (bottom right). The line shows the the cut for dilepton events $\left(H_{T}>200 \mathrm{GeV}\right)$. Only the events with $N_{j} \geq 2$ are selected for the final $t \bar{t}$ dilepton events. . . . . . . . . . . . . . 100

4.14 The $H_{T}$ of di-muon events after $E_{T}$ requirement in the $W W$ PYTHIA Monte Carlo sample, equivalent to $608.5 \mathrm{fb}^{-1}$. The plots are shown with no jet $\left(N_{j}=0\right.$, top left), $N_{j}=1$ (top right), $N_{j} \geq 2$ (bottom left), $N_{j} \geq 0$ (bottom right). The line shows the the cut for dilepton events $\left(H_{T}>200 \mathrm{GeV}\right)$. Only the events with $N_{j} \geq 2$ are selected for the final $t \bar{t}$ dilepton events. . . . . . . . . . . . . . . . 101 
4.15 The $H_{T}$ of $e$ - $\mu$ events after $E_{T}$ requirement in the $W W$ PYTHIA Monte Carlo sample, equivalent to $608.5 \mathrm{fb}^{-1}$. The plots are shown with no jet $\left(N_{j}=0\right.$, top left), $N_{j}=1$ (top right), $N_{j} \geq 2$ (bottom left), $N_{j} \geq 0$ (bottom right). The line shows the the cut for dilepton events $\left(H_{T}>200 \mathrm{GeV}\right)$. Only the events with $N_{j} \geq 2$ are selected for the final $t \bar{t}$ dilepton events. . . . . . . . . . . . . . . 102

5.1 The $H_{T}$ distributions of the six dilepton candidates, the expected backgrounds from $W W, Z^{0} \rightarrow \tau^{+} \tau^{-}$and $t \bar{t}$ Monte Carlo sample with $m_{t}=175 \mathrm{GeV} / \mathrm{c}^{2}$. The number of events for the backgrounds and $t \bar{t}$ Monte Carlo are normalized to 9 times the expected numbers. . . 116

5.2 The dilepton invariant mass distribution $\left(m_{\ell \ell}\right)$ of the six dilepton candidates. The distribution from the $t \bar{t}$ Monte Carlo sample $\left(m_{t}=\right.$ $175 \mathrm{GeV} / \mathrm{c}^{2}$ ) are also shown. The number of events for the $t \bar{t}$ Monte Carlo sample is normalized to 20 times the expected number. . . . . 117

5.3 The $E_{T}$ versus $\Delta \phi$ is plotted for the six candidate events and the $t \bar{t}$ Monte Carlo sample $\left(m_{t}=175 \mathrm{GeV} / \mathrm{c}^{2}\right) . \Delta \phi$ is the angle in the transverse plane between $E_{T}$ and the nearest lepton or jet. The dashed lines represent the $E_{T}$ cuts. . . . . . . . . . . . 118

A.1 The lepton identification variables of the central electron (TCE) obtained from the $Z^{0} \rightarrow e^{+} e^{-}$data sample (point) and a Monte Carlo sample (histogram). $E / p$ (top left), $E_{H A D} / E_{E M}$ (top right), Lshr (middle left), $\chi^{2}$ (middle right), charge $\times \Delta x$ (bottom left), $\Delta z$ (bottom right). . . . . . . . . . . . . . . . 132

A.2 The lepton identification variables of the plug electron (PEM) obtained from the $Z^{0} \rightarrow e^{+} e^{-}$data sample (point) and a Monte Carlo sample (histogram). $E_{H A D} / E_{E M}$ (top left), PEM $\chi_{3 \times 3}^{2}$ (top right), pesProfileRatio5by9(U) (bottom left), pesProfileRatio5by9(V) (bottom right). . . . . . . . . . . . . . . 133

A.3 The lepton identification variables of the central muon (CMUP) obtained from the $Z^{0} \rightarrow \mu^{+} \mu^{-}$data sample (point) and a Monte Carlo sample (histogram). $E_{E M}$ (top left), $E_{H A D}$ (top right), $\Delta x_{C M U}$ (bottom left), $\Delta x_{C M P}$ (bottom right) . . . . . . . . . . 134 
A.4 The lepton identification variables of the central muon (CMU) obtained from the $Z^{0} \rightarrow \mu^{+} \mu^{-}$data sample (point) and a Monte Carlo sample (histogram). $E_{E M}$ (top left), $E_{H A D}$ (top right), $\Delta x_{C M U}$ (bottom left). . . . . . . . . . . . . . . . 135

A.5 The lepton identification variables of the central muon (CMP) obtained from the $Z^{0} \rightarrow \mu^{+} \mu^{-}$data sample (point) and a Monte Carlo sample (histogram). $E_{E M}$ (top left), $E_{H A D}$ (top right), $\Delta x_{C M P}$ (bottom right). . . . . . . . . . . . . . 136

A.6 The lepton identification variables of the central muon (CMX) obtained from the $Z^{0} \rightarrow \mu^{+} \mu^{-}$data sample (point) and a Monte Carlo sample (histogram). $E_{E M}$ (top left), $E_{H A D}$ (top right), $\Delta x_{C M X}$ (bottom left). . . . . . . . . . . . . . . 137

A.7 The lepton identification variables of the minimum ionizing particle (CMIO) obtained from the $Z^{0} \rightarrow \mu^{+} \mu^{-}$data sample (point) and a Monte Carlo sample (histogram). $E_{E M}$ (top left), $E_{H A D}$ (top right), $E_{E M}+E_{H A D}$ (bottom left). . . . . . . . . . . . 138

B.1 The result of the pseudoexperiments (100,000 events) in $200 p b^{-1}$. The plots on the left are obtained from the step 1 procedure in the text. The plots on the right are obtained from the step 2 procedure in the text. The distributions of the cross sections are shown with a Gaussian fit. . . . . . . . . . . . . 146

B.2 The result of the pseudoexperiments (100,000 events) in $500 p b^{-1}$. The plots on the left are obtained from the step 1 procedure in the text. The plots on the right are obtained from the step 2 procedure in the text. The distributions of the cross sections are shown with a

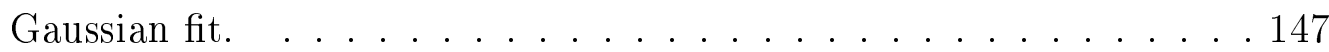

B.3 The result of the pseudoexperiments (100,000 events) in $2 \mathrm{fb}^{-1}$. The plots on the left are obtained from the step 1 procedure in the text. The plots on the right are obtained from the step 2 procedure in the text. The distributions of the cross sections are shown with a Gaussian fit. . . . . . . . . . . . . . . 148

C.1 Run 153325, Event 599511. . . . . . . . . . . . . . 150 
C.2 Run 153325, Event 599511. . . . . . . . . . . . . 151

C.3 Run 153374, Event 2276742. . . . . . . . . . . . . . . 152

C.4 Run 153374, Event 2276742. . . . . . . . . . . . . . . . . 153

C.5 Run 154654, Event 7344016. . . . . . . . . . . . . . . 154

C.6 Run 154654, Event 7344016. . . . . . . . . . . . . . 155

C.7 Run 155114, Event 478702. . . . . . . . . . . . . 156

C.8 Run 155114, Event 478702. . . . . . . . . . . . . . . 157

C.9 Run 156484, Event 3099305. . . . . . . . . . . . . . . 158

C.10 Run 156484, Event 3099305. . . . . . . . . . . . . . 159

C.11 Run 162820, Event 7050764. . . . . . . . . . . . . 160

C.12 Run 162820, Event 7050764. . . . . . . . . . . . . . 161 


\section{CHAPTER 1}

\section{INTRODUCTION}

\subsection{Overview of the Standard Model}

Modern particle physics, where fundamental particles and their interactions are the main interest, has been based on the "Standard Model" [1][2][3][4]. The Standard Model originates from requiring an invariance of a Lagrangian under the local gauge transformation of $\mathrm{SU}(3) \times \mathrm{SU}_{L}(2) \times \mathrm{U}_{Y}(1)$. The gauge bosons appear naturally to explain the interactions between the fundamental fermions in this model. The $W$, $Z$ and photon are gauge bosons which mediate the electroweak interaction between quarks and leptons. The gluons are gauge bosons for the strong interaction. The quarks carry one of three color charges, red, green or blue, for the strong interaction. These are named for reference and not related to the conventional meaning of colors. This model has been highly successful at explaining experimental observations over the last thirty years.

Weak interactions involving charged currents happen only in left-handed lepton pairs. These experimental facts lead to left-handed weak isospin doublets separate from right-handed singlets and imply that only the left-handed doublets couple to 
the $\mathrm{SU}_{L}(2)$ gauge field $W$. We can describe all known fundamental fermions participating in electroweak interactions, as shown in Table 1.1, with three generations for both leptons and quarks.

$$
\begin{aligned}
& \left(\begin{array}{c}
\nu_{e} \\
e^{-}
\end{array}\right)_{L}\left(\begin{array}{c}
\nu_{\mu} \\
\mu^{-}
\end{array}\right)_{L} \quad\left(\begin{array}{c}
\nu_{\tau} \\
\tau^{-}
\end{array}\right)_{L} \quad\left(\begin{array}{c}
u \\
d
\end{array}\right)_{L}\left(\begin{array}{l}
c \\
s
\end{array}\right)_{L}\left(\begin{array}{l}
t \\
b
\end{array}\right)_{L} \\
& \begin{array}{cccccc}
e_{R}^{-} & \mu_{R}^{-} & \tau_{R}^{-} & u_{R}, d_{R} & c_{R}, s_{R} & t_{R}, b_{R}
\end{array}
\end{aligned}
$$

Table 1.1: Quark and lepton $\mathrm{SU}_{L}(2)$ multiplets

Even though SU(2) doublets explain the charged current weak interactions involving two quarks within the same generation, experimental observations clearly show that there are also charged currents involving quarks between different generations. This implies a mixing between quarks in different generations. The quark members of the doublets appearing in the weak current operator should be different from the members of the quark mass eigenstates. The Cabibbo-Kobayashi-Maskawa (CKM) mixing matrix relates the quark members of these two different kinds of doublets $[3][4]$.

$$
\left(\begin{array}{c}
d^{\prime} \\
s^{\prime} \\
b^{\prime}
\end{array}\right)=\left(\begin{array}{ccc}
V_{u d} & V_{u s} & V_{u b} \\
V_{c d} & V_{c s} & V_{c b} \\
V_{t d} & V_{t s} & V_{t b}
\end{array}\right)\left(\begin{array}{c}
d \\
s \\
b
\end{array}\right)
$$

This matrix has a structure so that each orthogonal basis is related by a rotation to accommodate the 'GIM mechanism' [2]. This mechanism was proposed to explain 
the fact that no flavor-changing neutral current had been observed. This unitarity property constrains each element of this matrix. Within these constraints, there are several parameterizations for expressing this matrix [5]. The parameterizations have three angles and one phase. Each element of the matrix is expressed as a product of sines or cosines of the three angles and $e^{i \delta}$, where $\delta$ is the phase. This phase is directly related to the existence of CP (Charge conjugation and Parity) violation. The diagonal elements of the matrix are close to unity, which means that the weak current is dominant for quarks within the same generation. The weak currents between different generations are suppressed due to much smaller off-diagonal elements [5].

\subsection{Top Quark Physics}

The top quark is required in the standard model as the weak isospin partner of the $b$ quark. Indirect evidence for the existence of the top quark is found in the forward backward asymmetry, $A_{F B}$, in $b \bar{b}$ production from $e^{+} e^{-}$collisions. The asymmetry $A_{F B}$ depends on the weak isospin $T_{3 L}^{b}$ of the $b$ quark. It is zero if the $b$ quark is a weak isospin singlet but it is not zero if the $b$ quark is a member of the weak isospin doublet with $T_{3 L}^{b}=-1 / 2$. The first experimental result of the asymmetry in the scattering process $e^{+} e^{-} \rightarrow Z / \gamma \rightarrow b \bar{b}$ was obtained in the JADE collaboration at PETRA [6]. The JADE collaboration showed that $A_{F B}$ is consistent with the Standard Model value 0.0997 which is based on $T_{3 L}^{b}=-1 / 2$. A more recent measurement of $A_{F B}$ is $0.1002 \pm 0.0019$ [5] which strongly indicates that the $T_{3 L}=+1 / 2$ partner in the third generation of quarks exists. 
Another piece of indirect evidence for the top quark is the absence of the flavor changing neutral currents (FCNC) in $B$ decays. The branching ratio $B R\left(B^{0} \rightarrow\right.$ $\left.\mu^{+} \mu^{-}\right)<6.1 \times 10^{-7}[5]$ indicates that the FCNC transition is strongly suppressed and implies the extension of the GIM mechanism [2] to the third generation. The existence of an isodoublet partner of the $b$ quark can be inferred as an analogy of the charm quark which was required to cancel the FCNC transition.

The necessity of the third generation of quarks and the iso-partner of $b$ quark can be also sought from the renormalizability of the electroweak theory. The anomaly problem occurs in the triangle fermion loops involving the coupling of three electroweak bosons. The total amplitude of the loop is proportional to $\Sigma_{f} T_{3}^{f} Q_{f}^{2}$. This diverges if the contributions from all fermions do not cancel. The renormalizable electroweak theory requires the same number of generations in leptons and quarks as well as the same structure of electroweak isospins in both fermion types [7][8].

Direct evidence for the top quark had been sought by various experiments around the world. Since its mass was not predicted by the theory, it took a long time to find the signature of top quark production. Searches at the CERN $p \bar{p}$ collider with $\sqrt{s}=0.63 \mathrm{TeV}$ by the UA1 and UA2 collaborations had set the lower limit on the top mass of 60 and $69 \mathrm{GeV} / \mathrm{c}^{2}$ respectively [9][10]. At the Tevatron collider at Fermilab, the lower limit had been set at $91 \mathrm{GeV} / \mathrm{c}^{2}$ [11] and $131 \mathrm{GeV} / \mathrm{c}^{2}$ [12] with $\sqrt{s}=1.8 \mathrm{TeV}$.

These top quark searches assumed the Standard Model top quark decay to the $W$ boson and the $b$ quark. In $p \bar{p}$ collisions, the dominant top quark production takes place via $t \bar{t}$ pair production. The top quark production mechanism will be discussed 
in the next section in more detail. Assuming $t \bar{t}$ pair production, pairs of $W^{+} b$ and $W^{-} \bar{b}$ are produced. Since the top mass is greater than the sum of the masses of the $W$ boson and the $b$ quark, a real $W$ boson is produced. This $W$ boson then decays to a lepton and a neutrino, or a quark and an anti-quark. Therefore, depending on the $W$ decay mode, several different top production signatures exist. These decay channels from the top quark production will be discussed in the subsequent sections in more detail. Among various decay channels from the top quark production, the lepton + jets channel results from one $W$ boson decaying leptonically and the other hadronically. The dilepton channel has its signature from both $W$ bosons decaying leptonically. Also there is the all hadronic channel where both $W$ bosons decay hadronically. The top quark had been discovered using these channels in 1995 by the CDF and D $\varnothing$ collaborations at the Fermilab Tevatron collider [13][14]. The mass of the top quark was measured as $176 \pm 8$ (stat) \pm 10 (syst) $G e V / c^{2}$ and the

$t \bar{t}$ production cross section was measured as $6.8_{-2.4}^{+3.6} p b$ by the CDF collaboration [13]. The most recent value of the top quark mass is $174.3 \pm 5.1 \mathrm{GeV} / \mathrm{c}^{2}$ [5]. The cross section measurements of the $t \bar{t}$ production with $\sqrt{s}=1.8 \mathrm{TeV}$ at the Fermilab Tevatron are summarized in Table 1.2.

\subsection{Top Quark Production Mechanism}

In proton and anti-proton collisions at a center of mass energy $1.96 \mathrm{TeV}$, the dominant top quark production process is strong $t \bar{t}$ production. There are two prominent kinds of strong processes. One is quark-antiquark annihilation $(q \bar{q} \rightarrow t \bar{t})$ and the other is gluon fusion $(g g \rightarrow t \bar{t})$. Both processes are depicted in Figure 

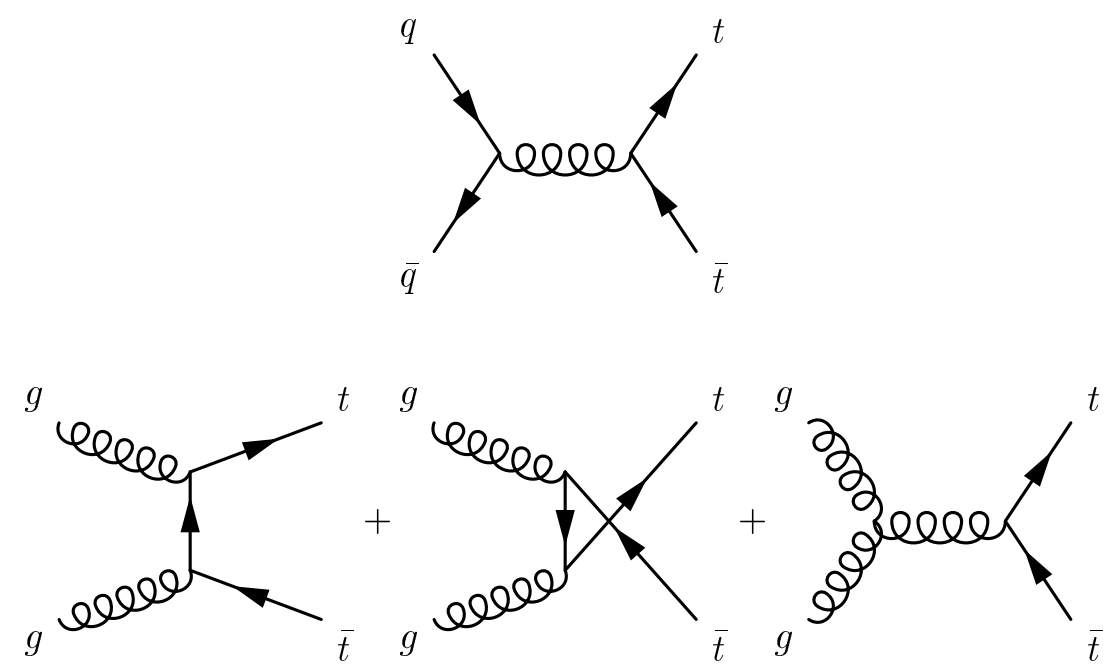

Figure 1.1: Lowest order Feynman diagrams for $t \bar{t}$ production via strong interaction in $p \bar{p}$ collisions.

1.1. Though top quark production via weak processes is also possible, these have relatively small cross sections compared to the strong processes [15]. The main focus of this thesis is $t \bar{t}$ production via the strong process.

The total cross section for heavy quark production in perturbative QCD is written in the form

$$
\sigma(p \bar{p} \rightarrow Q \bar{Q})=\sum_{i, j} \int_{0}^{1} d x_{1} \int_{0}^{1} d x_{2} f_{i}\left(x_{1}, \mu\right) f_{j}\left(x_{2}, \mu\right) \hat{\sigma}_{i j}\left(\hat{s}, m_{t}, \alpha_{s}(\mu)\right)
$$

where the sum is over the initial parton states. The function $f_{i}\left(x_{1}, \mu\right)$ is the probability distribution of a parton $i$ with a momentum fraction of $x_{1}$. The quantity $\mu$ 
is the renormalization scale introduced to avoid the infinities from the higher order Feynman diagrams. The quantity $\hat{\sigma}_{i j}$ is the total short distance cross section from parton $i$ and $j$ to produce a heavy quark pair. The value $\hat{s}$ is the center of mass energy of parton $i$ and $j$. The quantity $m_{t}$ is the mass of the heavy quark (in this case, the top quark). The function $\alpha_{s}(\mu)$ is the strong coupling constant which depends on $\mu$. In $p \bar{p}$ collisions at a center of mass energy of $1.96 \mathrm{TeV}$, about $85 \%$ of top pair productions are expected via quark-antiquark annihilation and $15 \%$ from gluon fusion. The calculation of the cross section for heavy quarks is sensitive to higher order corrections to the leading order calculation. The theoretical calculations in the next-to-leading-order (NLO) [16] at the center of mass energy $1.8 \mathrm{TeV}$ and 1.96 TeV are presented in Table 1.2 along with the previous experimental results from the CDF and DØ collaborations.

\begin{tabular}{cccc}
\hline & $\sqrt{s}$ & $m_{t}$ & $\sigma_{t \bar{t}}$ \\
\hline \hline CDF & $1.8 \mathrm{TeV}$ & $175 \mathrm{GeV} / \mathrm{c}^{2}$ & $7.6_{-1.5}^{+1.8} \mathrm{pb}$ \\
D & $1.8 \mathrm{TeV}$ & $173.3 \mathrm{GeV} / \mathrm{c}^{2}$ & $5.5 \pm 1.8 \mathrm{pb}$ \\
theoretical & $1.8 \mathrm{TeV}$ & $175 \mathrm{GeV} / \mathrm{c}^{2}$ & $5.19_{-0.68}^{+0.52} \mathrm{pb}$ \\
theoretical & $1.96 \mathrm{TeV}$ & $175 \mathrm{GeV} / \mathrm{c}^{2}$ & $6.70_{-0.88}^{+0.71} \mathrm{pb}$ \\
\hline
\end{tabular}

Table 1.2: The $t \bar{t}$ production cross section. CDF [18], D $\varnothing$ [21], and theoretical prediction [16]. The top quark masses $m_{t}$ in the table are used for the calculations of the cross section. 


\subsection{Top Quark Decay}

The Standard Model allows the top quark to decay into a real $W$ boson and a $b$ quark via the charged weak current. The probability that the top quark decays to other generations is strongly suppressed due to the negligible mixing expressed in the CKM matrix elements, in other words $V_{t b} \simeq 1$. The partial width for the top-quark decay [22] is expressed as;

$$
\begin{gathered}
\Gamma(t \rightarrow W b)=\frac{G_{F} M_{W}^{2}}{8 \pi \sqrt{2}} \frac{1}{m_{t}^{2}}\left|V_{t b}\right|^{2}\left[\frac{\left(m_{t}^{2}-m_{b}^{2}\right)^{2}}{M_{W}^{2}}+m_{t}^{2}+m_{b}^{2}-2 M_{W}^{2}\right] \times 2 k \\
k=\frac{\sqrt{\left(m_{t}^{2}-\left(M_{W}+m_{b}\right)^{2}\right)\left(m_{t}^{2}-\left(M_{W}-m_{b}\right)^{2}\right)}}{2 m_{t}}
\end{gathered}
$$

where $k$ denotes the $W$ momentum in the $t$ rest frame. With $M_{W}=80.4 \mathrm{GeV}$ and $m_{b}=5 \mathrm{GeV}$, the decay rate is $\Gamma(t \rightarrow W b) \simeq 1.55 \mathrm{GeV}$ and the lifetime is $\tau_{t} \sim 4 \times 10^{-25} \mathrm{sec}$

A very short lifetime of the top quark means that it decays like a free quark. The top quark decays via the charged electroweak current before it fragments and forms hadrons. This characteristic and the constraint from $V_{t b}$ allow a unique signature of the top-quark decay to $b$ quarks and $W$ bosons. The $b$ quark has a long lifetime and $b$ hadrons can travel long distances before they decay. Since a quark is a colored object, once the $b$ quark is produced, it cannot exist as a free particle. So quarkantiquark pairs are created to make colorless bound states including a $b$ hadron through a process called hadronization. The hadrons created as a result of this process tend to travel together in a "jet" along the direction of the original quark. 
Techniques have been developed to identify the decay vertex of long-lived $b$ hadrons in jets. Jets with these decay vertices are said to be "b tagged" and the jets are considered as coming from $b$ quarks. The other decay product from the top quark, the $W$ boson, decays to a lepton and neutrino pair or hadronically to a pair of light quarks, which then hadronize to form jets.

Due to the different decay modes of the $W$ bosons, $t \bar{t}$ decays can be categorized in three main classes (Table 1.3). In the dilepton channel, both $W$ bosons from the $t \bar{t}$ pair decay into leptons and neutrinos. In the final state of this channel, there are two highly energetic leptons, a large missing energy from the unobserved neutrinos, and two jets from $b$ decays (Figure 1.2). For this study, the decay channel involving the $\tau$ lepton is not considered because the $\tau$ lepton subsequently decays and leaves a different experimental signature. Due to the difficulties in the observation of $\tau$, the detection efficiency of decay channel involving $\tau$ lepton is very small. In the previous studies on the dilepton channel of $t \bar{t}$ production involving one $\tau(e \tau, \mu \tau)$, the detection efficiency is only $18 \%$ of the dilepton channel involving only electrons or muons (first three classes in Table 1.3) [17]. The dilepton channel comprises 5\% of the total decay rate. This channel benefits from the clean signature of the final state, which makes separating the $t \bar{t}$ signal from the backgrounds easier even though the branching ratio is smaller than other channels.

In the lepton + jets channel, one of the $W$ boson decays hadronically and the other decays to a lepton and a neutrino. The lepton + jets channel involves a high $p_{T}$ lepton, a large missing energy from a neutrino, and four jets. In this analysis, usually at least one jet is required to be identified as a $b$ jet using the displaced vertex 


\begin{tabular}{c|c|c|c}
\hline Category & Decay mode & \multicolumn{2}{|c}{ Branching ratio } \\
\hline \hline & $t \bar{t} \rightarrow e \nu b e \nu \bar{b}$ & $1 / 81$ & \\
Dilepton & $t \bar{t} \rightarrow \mu \nu b \mu \nu \bar{b}$ & $1 / 81$ & $4 / 81 \quad(5 \%)$ \\
$(e, \mu)$ & $t \bar{t} \rightarrow e \nu b \mu \nu \bar{b}$ & $2 / 81$ & \\
\hline & $t \bar{t} \rightarrow e \nu b \tau \nu \bar{b}$ & $2 / 81$ & \\
Dilepton & $t \bar{t} \rightarrow \mu \nu b \tau \nu \bar{b}$ & $2 / 81$ & \\
$($ with $\tau)$ & $t \bar{t} \rightarrow \tau \nu b \tau \nu \bar{b}$ & $1 / 81$ & \\
\hline \hline Lepton & $t \bar{t} \rightarrow q \bar{q} b e \nu \bar{b}$ & $12 / 81$ & $24 / 81(30 \%)$ \\
+ jets & $t \bar{t} \rightarrow q \bar{q} b \mu \nu \bar{b}$ & $12 / 81$ & \\
\hline$\tau+$ jets & $t \bar{t} \rightarrow q \bar{q} b \tau \nu \bar{b}$ & $12 / 81$ & \\
\hline \hline All hadronic & $t \bar{t} \rightarrow q \bar{q} b q \bar{q} \bar{b}$ & $36 / 81$ & $36 / 81(44 \%)$ \\
\hline
\end{tabular}

Table 1.3: $t \bar{t}$ decay modes and their branching ratios.

information as discussed above. About $30 \%$ of the total decay rate comes from this channel. The all hadronic channel has the biggest branching ratio (44\%). However, it has difficulties in extracting the $t \bar{t}$ signal from the large QCD backgrounds.

\subsection{Dilepton Decay Channel}

Among three $t \bar{t}$ decay channels described in the previous section, the dilepton decay channel is used for the cross section measurement in this thesis. The dilepton channel overcomes the shortcoming of the small branching fraction with its high signal purity. Because the dilepton channel relies on the identification of two high $p_{T}$ leptons, it is possible to find the $t \bar{t}$ candidates more clearly than other channels that depend more on the measurement of jets. Generally, the measurement of 


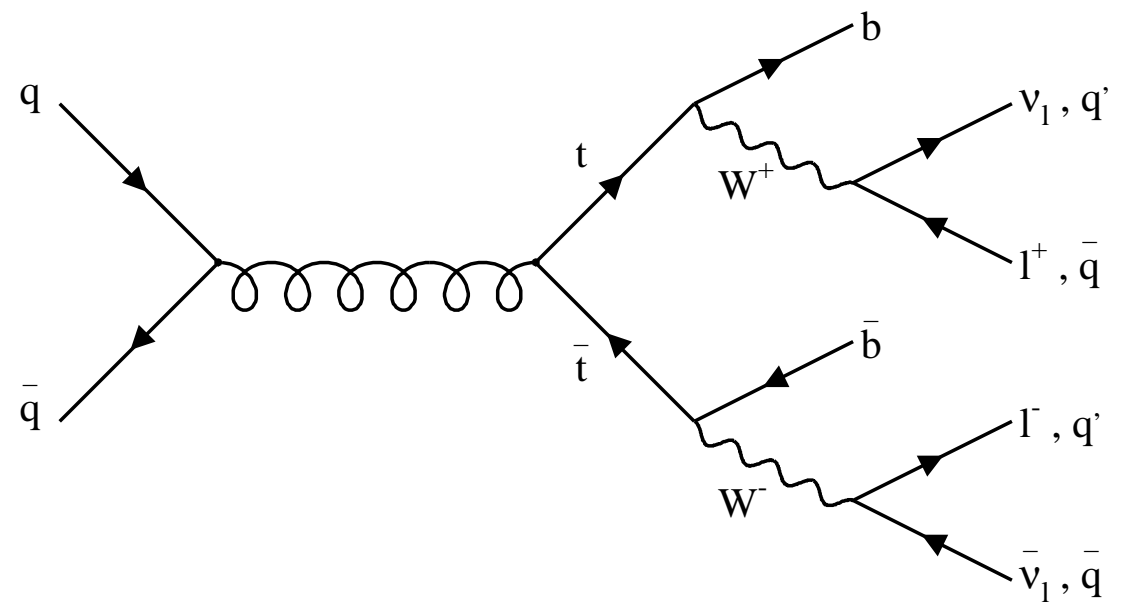

Figure 1.2: $q \bar{q} \rightarrow t \bar{t}$ production and Standard Model $t \bar{t}$ decay. 


\begin{tabular}{ccc}
\hline$t \bar{t}$ cross section & Source & Method \\
\hline \hline $6.7_{-1.7}^{+2.0} p b$ & CDF & lepton + jets \\
$8.2_{-3.4}^{+4.4} p b$ & CDF & dileptons \\
$10.1_{-3.6}^{+4.5} p b$ & CDF & all jets \\
\hline $7.6_{-1.5}^{+1.8} p b$ & CDF & three methods combined \\
\hline
\end{tabular}

Table 1.4: Cross section measurements using different $t \bar{t}$ decay channels in $p \bar{p}$ collisions at $\sqrt{s}=1.8 \mathrm{TeV}$ from the CDF collaboration $\left(m_{t}=175 \mathrm{GeV} / \mathrm{c}^{2}\right)$ [18][19][20].

electrons and muons are much more reliable than that of jets. Furthermore, the backgrounds of the $t \bar{t}$ dilepton channel can be efficiently removed by the unique finalstate signature of two high $p_{T}$ leptons, a large missing energy and two hadronic jets. Using these features, it provides a high signal-to-background ratio which is crucial for reducing the uncertainty of the cross section measurement. The Run I CDF collaboration results of the cross section measurements of the $t \bar{t}$ production in three different $t \bar{t}$ decay channels are shown in Table 1.4.

\subsection{Overview of thesis}

This thesis describes the measurement of the $t \bar{t}$ production cross section in proton and anti-proton collisions at a center of mass energy $1.96 \mathrm{TeV}$. The analysis uses the dilepton decay channel where two highly energetic leptons, 2 jets from the 
fragmentation of the $b$ quarks and a large missing energy from the unobserved neutrinos are the signature of the decay. In Chapter 2, the experimental apparatus is briefly introduced. This chapter describes the general aspects of the CDF detector and new features which have been upgraded from the previous Run I detector.

The dilepton channel acceptance, the probability of the $t \bar{t}$ event passing the dilepton selection requirements, is discussed in Chapter 3. A Monte Carlo $t \bar{t}$ sample is generated and the dilepton selection requirements are applied to the sample. The dilepton channel acceptance is the ratio of the number of events passing the requirements to the number of events of the original sample. The dilepton selection requirements exploit the unique aspects of the dilepton decay channel of $t \bar{t}$ production. It requires the event to have two jets from the $b$ quark fragmentations. A large missing energy is required to ensure the neutrinos originated from the leptonic decays of the $W$ bosons. The large total transverse energy is also a unique characteristic of $t \bar{t}$ production. The total dilepton channel acceptance of $0.43 \pm 0.04 \%$ is obtained.

In Chapter 4, the methods used to remove the backgrounds from the $t \bar{t}$ signal will be discussed. The main backgrounds include the Drell-Yan process, di-boson production, and $Z^{0} \rightarrow \tau^{+} \tau^{-}$. These backgrounds have very similar final state with two leptons as the $t \bar{t}$ production in the dilepton channel. However, they generally do not involve jets in their lowest order process. Furthermore, they show differences in some of aspects used for the dilepton selection requirements. The expected backgrounds in $109 p b^{-1}$ of data are also estimated as $0.65 \pm 0.13$ events. 
In Chapter 5 , the candidates of the $t \bar{t}$ production in the dilepton channel are sought. In this search, one $e e$, three $\mu \mu$, and two $e \mu$ candidates are found in data of $109 p b^{-1}$. Using these numbers and the estimates of the acceptance and backgrounds, the cross section of the $t \bar{t}$ production is calculated. The cross section obtained from this calculation is $\sigma_{t \bar{t}}=11.4_{-4.6}^{+6.2} \mathrm{pb}$. Finally, Chapter 6 summarizes this thesis. 


\section{CHAPTER 2}

\section{EXPERIMENTAL APPARATUS}

\subsection{The Tevatron Collider}

The Tevatron collider at Fermi National Accelerator Laboratory (Fermilab) provides proton and anti-proton collisions at a center-of-mass energy of $1.96 \mathrm{TeV}$. A number of important discoveries have been made at Fermilab since it was commissioned in 1967. Three standard model particles were first observed using the Fermilab accelerators. The most recent one was the direct observation of the $\nu_{\tau}$ (tau neutrino) [24]. In 1977, using the Main Ring, the bottom quark was discovered. The Main Ring was the predecessor to the Tevatron and it could accelerate protons to $400 \mathrm{GeV}$. It was located in the same tunnel where the Tevatron currently resides. Until 1997, the Main Ring was used to accelerate the protons before they were injected into the Tevatron. The Tevatron, which was the first accelerator using superconducting magnets, began operating in 1983 with a beam energy of $512 \mathrm{GeV}$. The Tevatron's beam energy was gradually increased up to $900 \mathrm{GeV}$ in 1986 . In 1995, the top quark was first observed at the Tevatron by the CDF and DØ collab- 
orations [13][14]. In 1997, the accelerator complex was temporarily shutdown for upgrades. This included the introduction of the main injector to replace the role of the main ring.

The proton accelerator chain is initiated in the Cockcroft-Walton pre-accelerator. The $H^{-}$ions prepared from hydrogen gas are accelerated inside this device and reach an energy of $750 \mathrm{keV}$. Next, the ions are accelerated by a linear accelerator (Linac) to $400 \mathrm{MeV}$. Before they are injected into the circular Booster, the ions pass through a carbon foil which removes the electrons, leaving the proton nucleus. In the Booster, the protons repeatedly experience electric fields in the circular path attaining an energy of $8 \mathrm{GeV}$ and are coalesced into bunches. The protons enter the Main Injector, which is a new accelerator introduced in 1999, and are accelerated to $150 \mathrm{GeV}$. In this step, a portion of protons with $120 \mathrm{GeV}$ are transferred to the antiproton source. In the antiproton source, the protons collide with a nickel target. The antiprotons among the secondary particles of the collision are then sent to the accumulator ring and subsequently to the Main Injector. The protons and antiprotons with $150 \mathrm{GeV}$ energy are sent to the Tevatron where they are accelerated to $980 \mathrm{GeV}$.

The luminosity is a measure of the intensity of the beams. It depends on the number of protons in a bunch $\left(N_{p}\right)$, the number of antiprotons in a bunch $\left(N_{\bar{p}}\right)$, the number of bunches of each type of particle $(B)$, the revolution frequency of the bunches $\left(f_{0}\right)$, and the transverse cross-sectional area of the bunches $(\sigma)$. The instantaneous luminosity is given by Equation 2.1.

$$
\mathcal{L}=\frac{N_{p} N_{\bar{p}} B f_{0}}{4 \pi \sigma}
$$



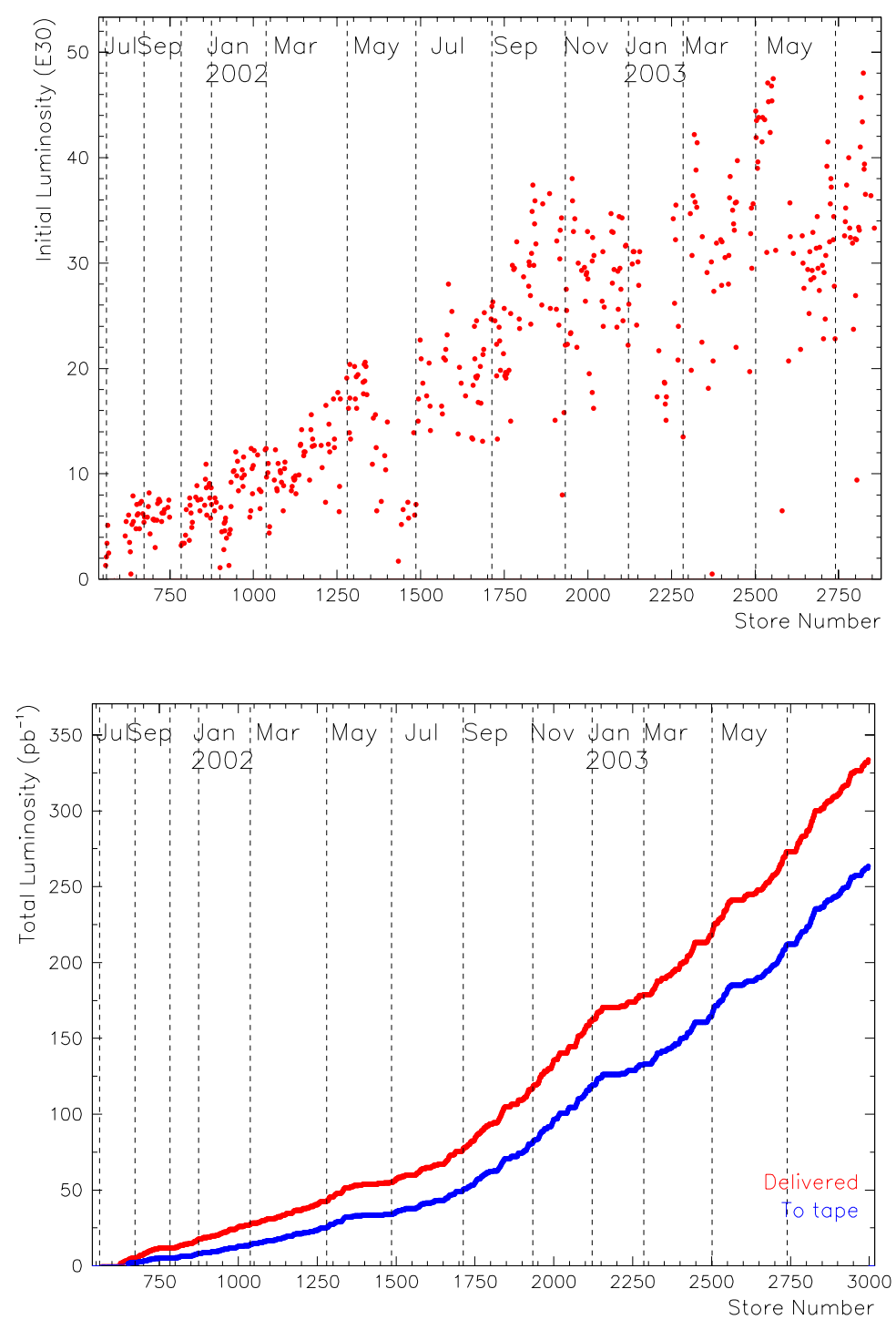

Figure 2.1: The instantaneous luminosity (top) and integrated luminosity (bottom) of the Tevatron since July 2001. 
In Run II which started in 2001, the goal of accelerator operation was $\mathcal{L}=8 \times$ $10^{31} \mathrm{~cm}^{-2} \mathrm{~s}^{-1}$ [26]. As of August 2003, the typical luminosity is $\mathcal{L}=2 \times 10^{31} \mathrm{~cm}^{-2} \mathrm{~s}^{-1}$ and the best luminosity was $\mathcal{L}=4.8 \times 10^{31} \mathrm{~cm}^{-2} \mathrm{~s}^{-1}$ [27]. The luminosity has been continuously increased as shown in Figure 2.1. In Run IB (until 1995), the typical value was $1.6 \times 10^{31} \mathrm{~cm}^{-2} \mathrm{~s}^{-1}$ and the best one was $2.8 \times 10^{31} \mathrm{~cm}^{-2} \mathrm{~s}^{-1}$. The goal of the increased luminosity in Run II is attainable mainly from the increase of proton and antiproton bunches from 6 in Run IB to 36 which consequently results in shorter bunch spacing from $3.5 \mu$ sec to 396 nsec.

\subsection{The CDF Detector}

The CDF detector was designed for various studies of high energy physics from proton and antiproton collisions. It is located in one of the interaction points of the Tevatron. The schematic diagram of the components of the CDF detector is depicted in Figure 2.2. The CDF detector is cylindrically symmetric with the axis along the Tevatron beam-pipe and forward-backward symmetric about the transverse plane passing through the nominal interaction point. As a convention, the coordinate system of the CDF detector is defined with the positive $z$ direction as the direction of the proton beam, the positive $y$ direction as upward from the detector and the positive $x$ direction as outward from the center of the Tevatron. A more commonly used coordinate system is $\phi-\eta$ system, where $\phi$ is the azimuthal angle. The pseudorapidity $(\eta)$ is defined by Equation 2.2, where $\theta$ is the polar angle measured relative to the positive $z$ direction.

$$
\eta=-\ln (\tan (\theta / 2))
$$




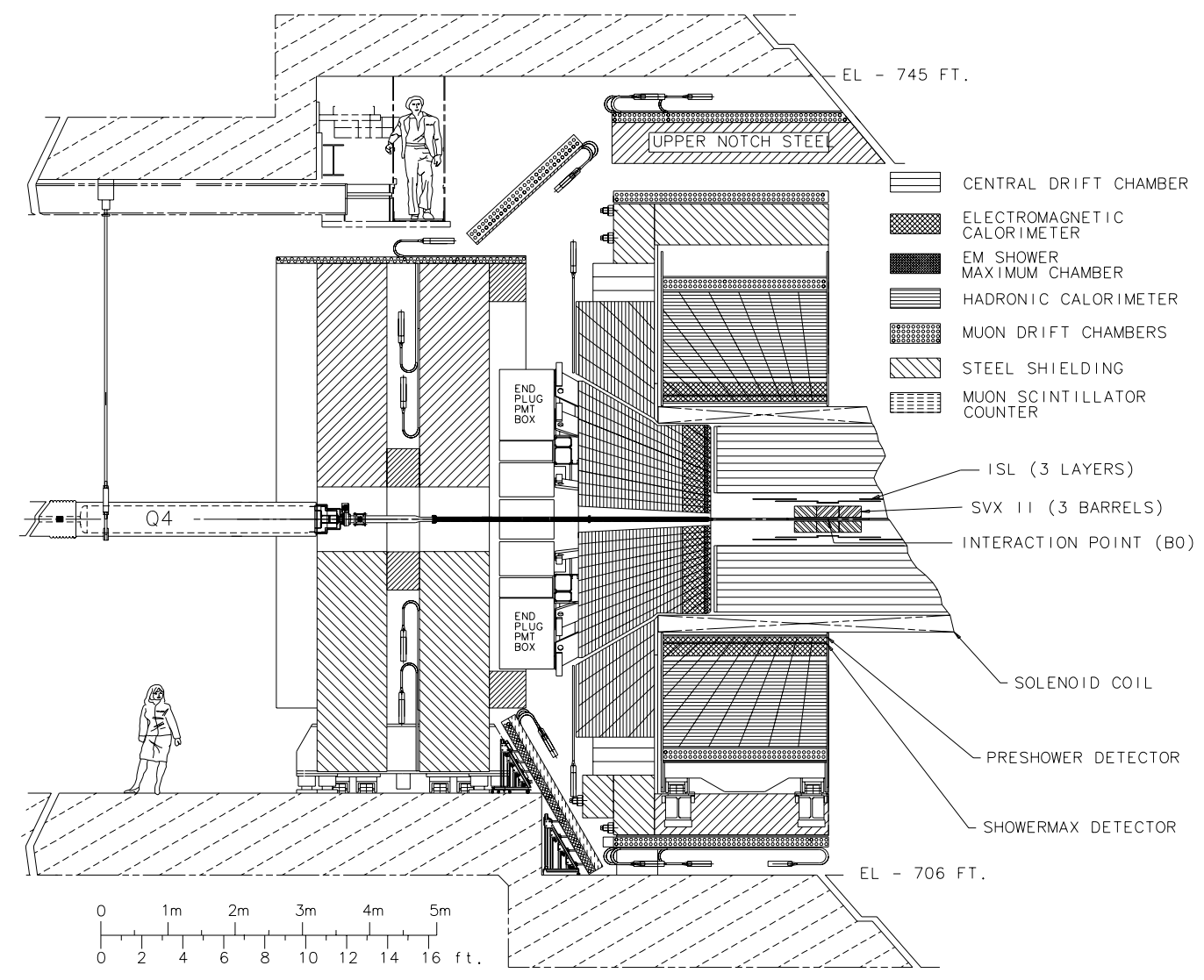

Figure 2.2: An elevation view of one half of the CDF II detector. 
Among the various components of the CDF detector, the three parts which are the most important for this analysis are the tracking system, calorimeters and muon detector system. The central tracking system is housed inside the solenoid. Exploiting the curved track due to the magnetic field provided by the solenoid, the tracking system measures the momentum and charge of the particles. The calorimeters measure the energy of the electron, photon, and jets in the outer radius of the tracking system. The muon drift chambers detect muons escaping from the calorimeters and steel absorbers. These components will be discussed in more detail in the following sections. More complete descriptions of the CDF run II detector can be found elsewhere [28].

\subsubsection{The Tracking System}

In Run I, the tracks of the charged particles were measured by the Central Tracking Chamber (CTC), the Silicon Vertex Detector (SVX), and the Vertex Time Projection Chamber (VTX). However, in the environment of the higher luminosity and shorter bunch spacing in Run II, a significant modification in the design of these components was required. Also, the Run I Silicon Vertex Detector had limitation in its acceptance due to its short length. The CTC was replaced by the Central Outer Tracker (COT). The Silicon Vertex Detector II (SVX II) replaced the SVX.

Particle tracking from a radius of $40 \mathrm{~cm}$ to $137 \mathrm{~cm}$ and $|\eta|<1.0$ is performed by the Central Outer Tracker (COT). The COT is a set of wire drift chambers filled with Ar-Ethan- $C F_{4}$ gas. In comparison with the CTC, the size of an individual drift cell is smaller and the gas has a shorter drift time which is required by the shorter 
bunch spacing of Run II. The COT is designed to operate with a maximum drift of 100 nsec. The COT has two types of wires: axial and stereo. The axial wires are arranged parallel to the beam-pipe for the measurement of the momentum in the $r-\phi$ direction. The stereo wires are slightly tilted with respect to the axial wires for the measurement in the $r-z$ direction. In Run I, the stereo measurement was poor because the CTC had 24 stereo wires out of the 84 wires. The COT has 48 stereo wires which provide an enhanced performance in the $r-z$ reconstruction. The COT has 8 superlayers in which 12 wire drift cells are tilted at $35^{\circ}$ with respect to the radial direction. This compensates for the Lorentz angle and makes the ionization electrons drift perpendicular to the radial direction. Among the 8 superlayers, 4 layers are axial, which is parallel to the beam line, and the other 4 layers are stereo with a $3^{\circ}$ offset. A total of 96 measurements are provided for a charged track by the COT. The sideview of the arrangement of the COT drift cells is shown in Figure 2.3. The mechanical properties of the COT are listed in Table 2.1.

The Silicon Vertex Detector (SVX) was first introduced in 1992 to detect the vertices from heavy flavor weak decays. It has a crucial role in the reconstruction of vertices from the $B$ hadron decays for the study of the top quark. Originally the SVX consisted of two barrels with a total length of $51 \mathrm{~cm}$, aligned along the beampipe. There were 12 wedges in a barrel with 4 layers. Each layer in a wedge is called a ladder and had single sided silicon-strip detectors. In Run I, only $r-\phi$ measurements were possible because all the silicon strips were aligned with the beam direction. In Run II, the SVX was upgraded to allow more acceptance and higher precision measurement of vertices. The SVX II has three barrels with a total 


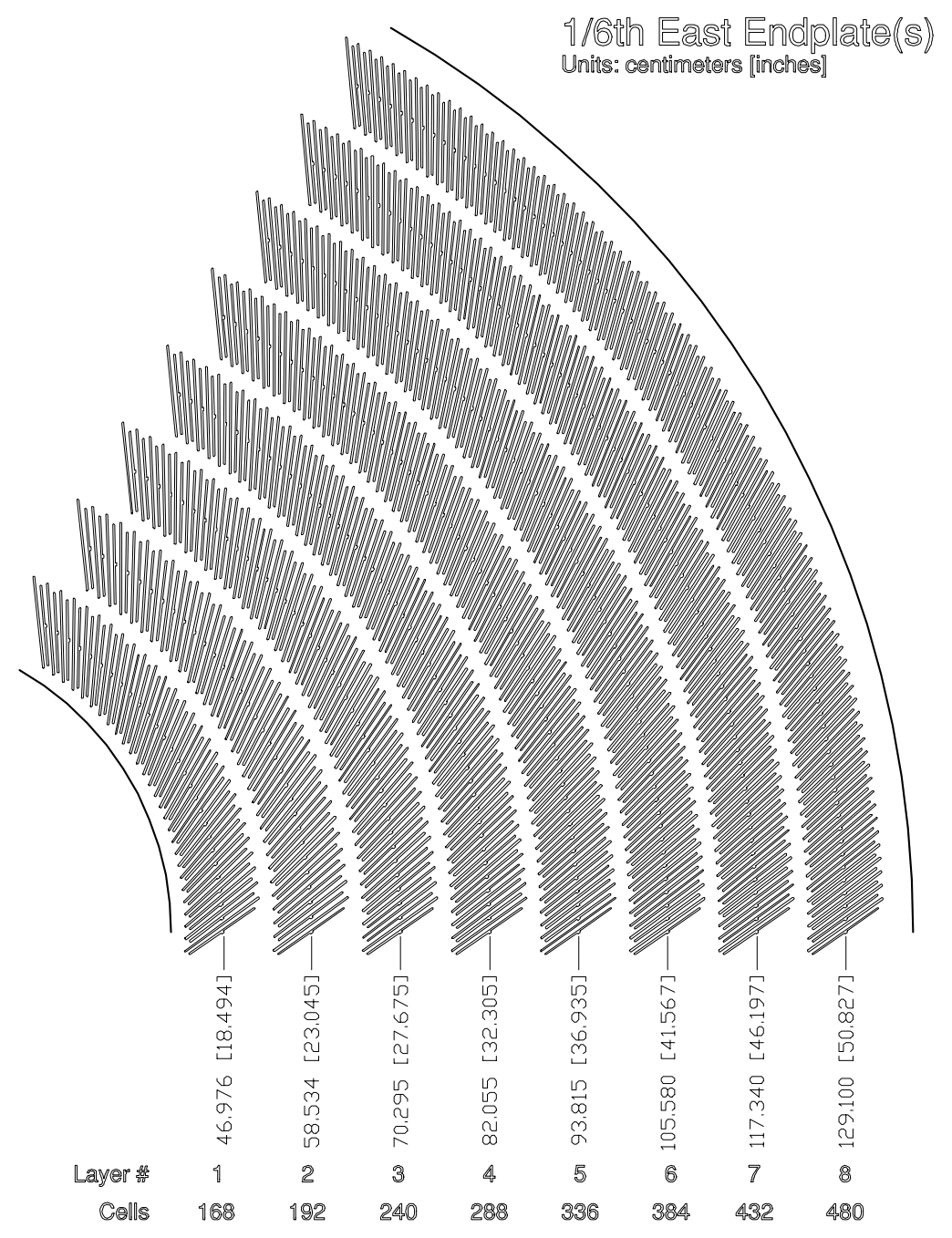

Figure 2.3: The arrangement of drift cells at the east endplate of the COT. Only $1 / 6$ portion in $\phi$ is shown. 


\begin{tabular}{ll}
\hline \hline Number of Layers & 96 \\
Number of Super layers & 8 \\
Stereo Angle & $+3,0,-3,0,+3,0,-3,0^{\circ}$ \\
Cells/Layers & $168,192,240,288,336,384,432,480$ \\
Sense wires/Cell & $12,12,12,12,12,12,12,12$ \\
Radius at Center of SL & $46,58,70,82,94,106,119,131 \mathrm{~cm}$ \\
Sense wire Spacing & $0.3 \prime \prime(7.62 \mathrm{~mm})$ in plane of wires \\
Wire material & gold plated Tungsten \\
Wire tension & $135 \mathrm{~g}$ \\
Tilt Angle & $35^{\circ}$ \\
Length of Active Region & $310 \mathrm{~cm}$ \\
Total number of Wires & 63000 \\
Endplate Load & $\sim 40 \mathrm{tons}$ \\
Drift Field & $\sim 2.5 \mathrm{kV} / \mathrm{cm}$ \\
\hline \hline
\end{tabular}

Table 2.1: Description of the Central Outer Tracker (COT). 


\begin{tabular}{lcc}
\hline \hline Detector Parameter & SVX & SVX II \\
\hline Readout coordinates & $r-\phi$ & $r-\phi, r-z$ \\
Number of barrels & 2 & 3 \\
Number of layers per barrels & 4 & 5 \\
Number of wedges per barrels & 12 & 12 \\
Ladder length & $25.5 \mathrm{~cm}$ & $29.0 \mathrm{~cm}$ \\
Combined barrel length & $51.0 \mathrm{~cm}$ & $87.0 \mathrm{~cm}$ \\
Layer geometry & $3^{\circ} \mathrm{tilt}$ & staggered radii \\
Radius innermost layer & $3.0 \mathrm{~cm}$ & $2.44 \mathrm{~cm}$ \\
Radius outermost layer & $7.8 \mathrm{~cm}$ & $10.6 \mathrm{~cm}$ \\
$r-\phi$ readout channels & 46,080 & 211,968 \\
$r-z$ readout channels & absent & 193,536 \\
Total number of channels & 46,080 & 405,504 \\
Total number of detectors & 288 & 720 \\
Total number of ladders & 96 & 180 \\
\hline \hline
\end{tabular}

Table 2.2: Comparison of SVX and SVX II.

length of $87 \mathrm{~cm}$, therefore it increases the geometric acceptance for finding the $B$ hadron decay vertices from $60 \%$ in Run I to almost $100 \%$. Each barrel in the new detector has five layers which have double-sided silicon-microstrip detectors. One side of the silicon detectors is for an $r-\phi$ measurement. The other side is for an $r-z$ measurement with $90^{\circ}$ or small angle stereo layers. The details of the SVX II detector are shown in Table 2.2 along with a comparison to the Run I SVX.

The Intermediate Silicon Layers (ISL) is a new addition to the CDF detector in Run II. It is placed outside the SVX II detector and covers the radial region from $20 \mathrm{~cm}$ to $28 \mathrm{~cm}$. The ISL consists of three layers which have different length in the $z$ direction. In the region $1.0<|\eta|<2.0$, two layers at the radii of $20 \mathrm{~cm}$ and $28 \mathrm{~cm}$ 
provide complete $3 \mathrm{D}$ tracking capability where the COT coverage is incomplete or missing. In the central region of $|\eta|<1.0$, a single layer of silicon is placed at a radius of $22 \mathrm{~cm}$. The 6 layers from SVX and ISL in the central region provide higher tracking efficiency as well as better momentum resolution in conjunction with the COT. The regions covered by the tracking systems in $\eta$ are shown in Figure 2.4. An end view of the CDF silicon detector systems is shown in Figure 2.5. The expected momentum resolution of the CDF tracking system when the COT, SVX, ISL are combined, is $\delta p_{T} / p_{T}^{2} \leq 0.001 \mathrm{GeV}^{-1} c$.

\subsubsection{Calorimetry}

The calorimeters of the CDF, are located outside of the solenoid. In different $\eta$ regions, there are three kinds of calorimeters installed. The central calorimeters consist of the central electromagnetic calorimeter (CEM), the central hadronic

calorimeter (CHA) and the wall hadronic calorimeter (WHA). They cover $|\eta|<1.1$, $|\eta|<0.9$, and $0.7<|\eta|<1.3$ respectively. In the higher $\eta$ region, the plug electromagnetic calorimeter (PEM) and the plug hadronic calorimeter (PHA) provide the coverage of $1.1<|\eta|<3.6$.

The central calorimeters are segmented into 24 wedges, each covering $15^{\circ}$ in azimuth, extending $250 \mathrm{~cm}$ from $z=0$ to either side of the detector. Each wedge of the electromagnetic calorimeter consists of an alternating lead-scintillator material. Incident particles interact with absorbing material (lead) and lose a fraction of energy while creating cascades of secondary particles. In the scintillator layer, the particles produce light which is captured by a photomultiplier tube (PMT). The 


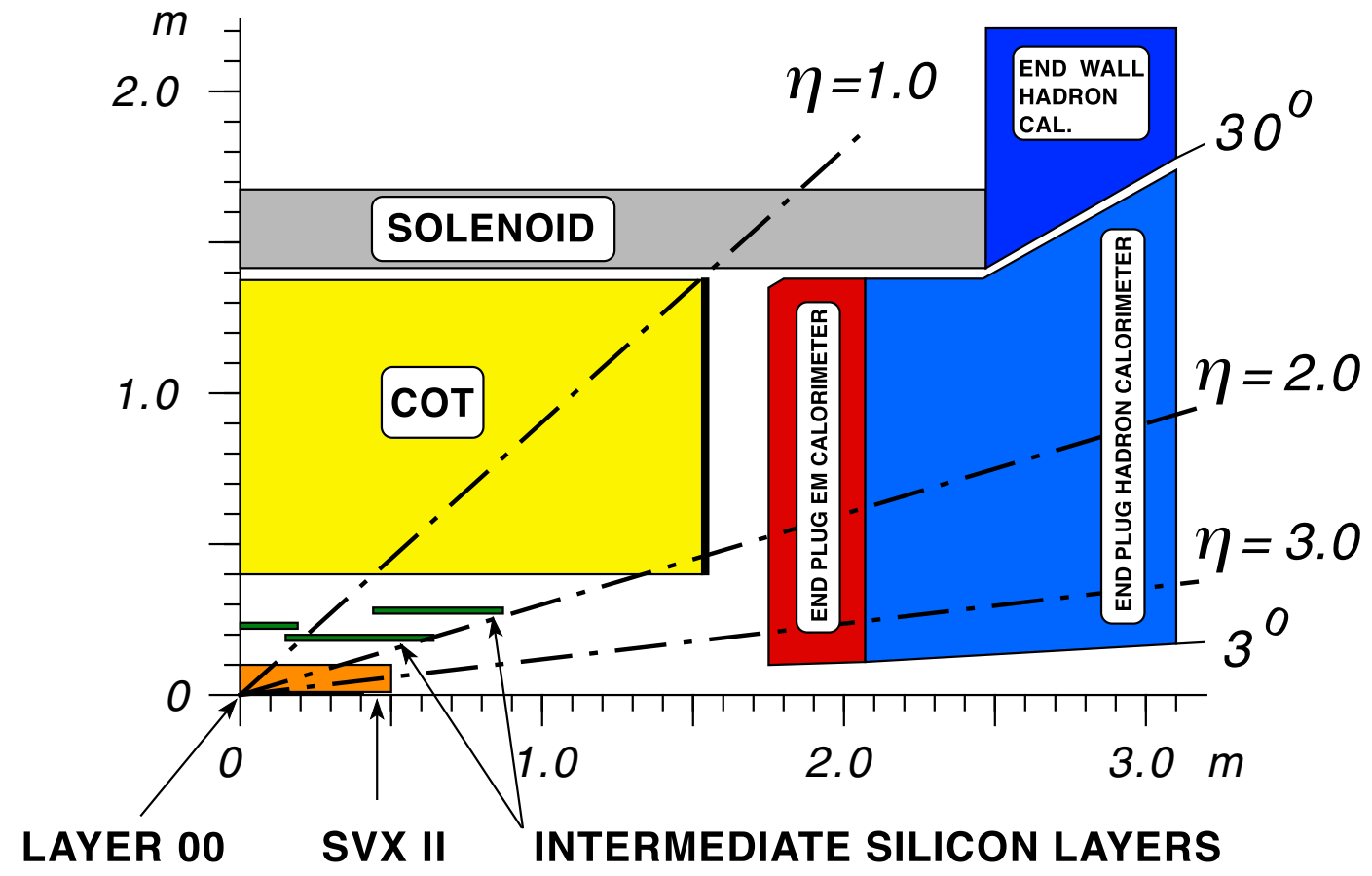

Figure 2.4: A schematic view of CDF run II detector showing the $\eta$ coverage of the tracking systems surrounded by the solenoid and the plug calorimeters. 


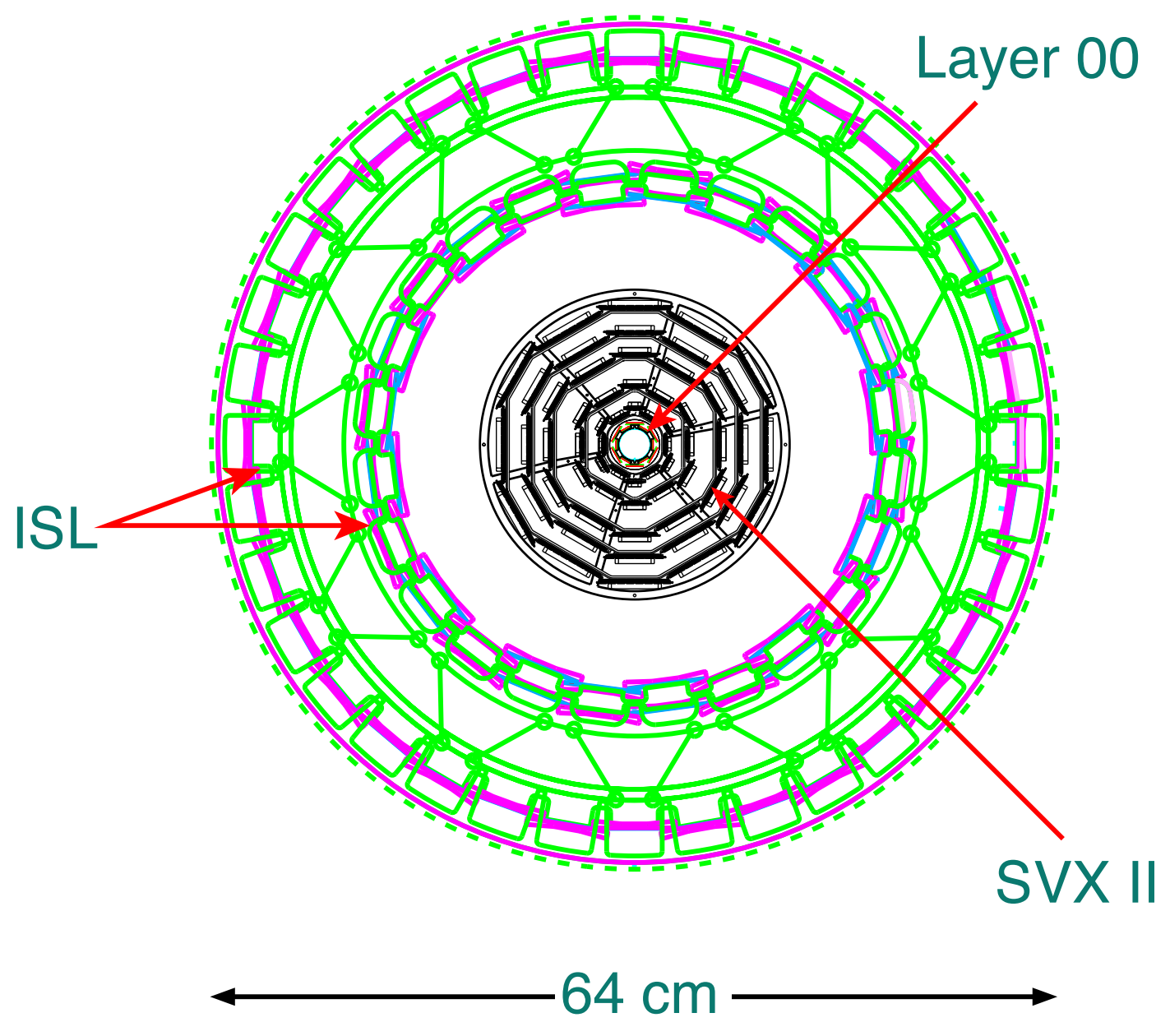

Figure 2.5: An end view of the CDF silicon system including the SVX II bulkheads and ISL support structure. 
hadronic calorimeter which uses steel-scintillator is located behind the electromagnetic calorimeter because a hadronic shower develops later than an electromagnetic shower. The central electromagnetic shower counter (CES) is located at 5.9 radiation lengths where the maximal average electromagnetic shower development occurs. The CES is a proportional strip and wire chamber which provides shower position in the $z-\phi$ plane.

The CEM has 18 radiation lengths worth of material and an inner radius of $173 \mathrm{~cm}$ with a depth of $35 \mathrm{~cm}$. The CHA contains 4.7 absorption lengths of material and extends beyond the CEM. The layout of the central calorimeter is shown in Figure 2.7. The measured energy resolution for electromagnetic showers is $13.7 \% / \sqrt{E_{T}} \oplus 2 \%$ where $\oplus$ means an addition in quadrature. The CEM was calibrated using a testbeam of electrons and is checked periodically using radioactive ${ }^{137} \mathrm{Cs}$ sources. The energy resolution for hadronic showers was measured from isolated pions; the resolution in the central hadronic calorimetry was determined to be $50 \% / \sqrt{E_{T}} \oplus 3 \%$. Table 2.3 lists detailed characteristics of the central calorimeters. A cross-section view of a central calorimeter wedge is shown in Figure 2.6.

The plug calorimeters are one of the most important improvements of the Run II detector. This upgraded calorimeter covers the polar angle region from $37^{\circ}$ to $3^{\circ}$, $(1.1<|\eta|<3.6)$ replacing the gas calorimeter of Run I, whose time response would have poorly matched the operational conditions of Run II. The plug electromagnetic calorimeter has lead-scintillator alternating layers with $4.5 \mathrm{~mm}$ of lead and $4 \mathrm{~mm}$ of 


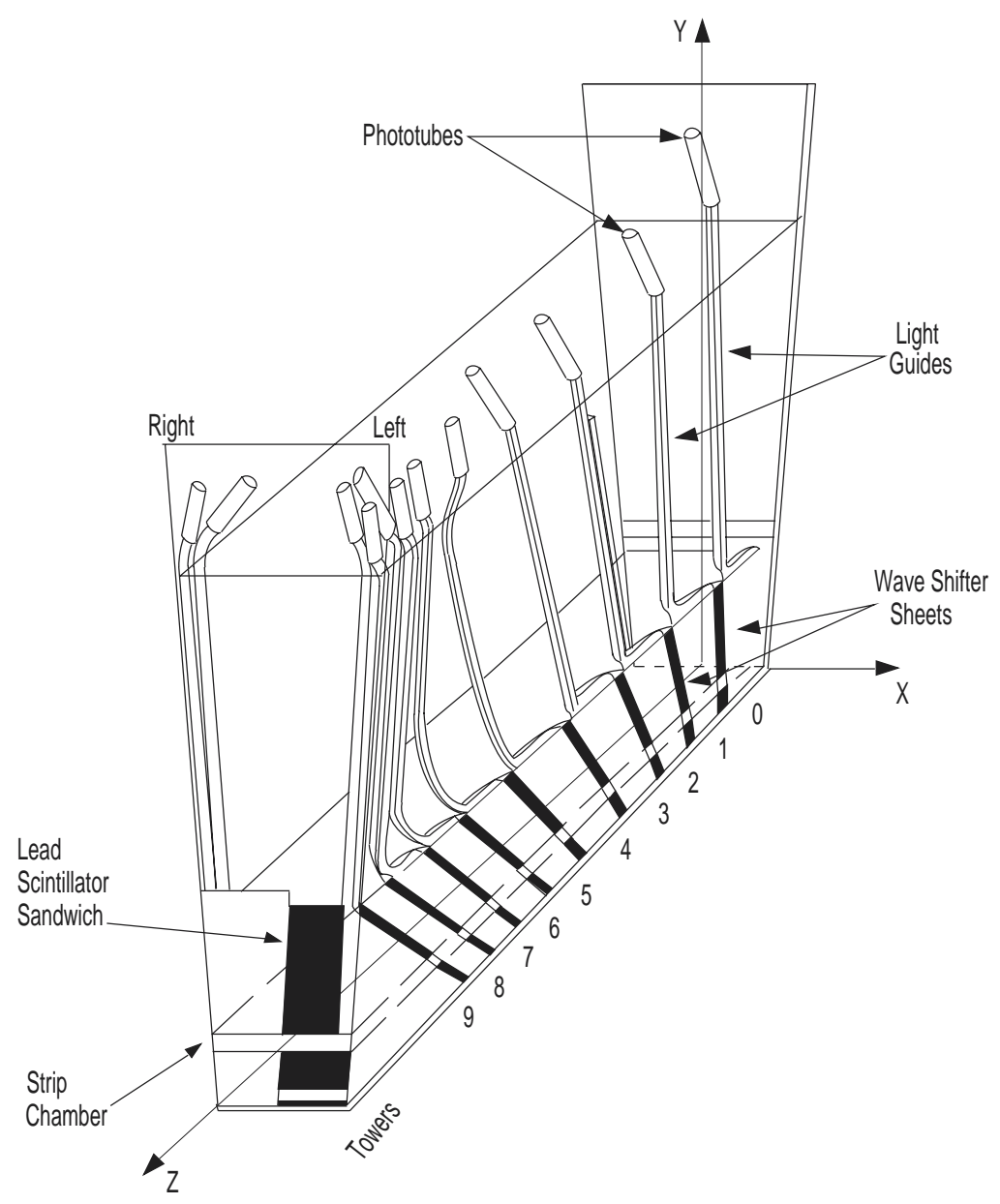

Figure 2.6: Diagram of a single central calorimetry wedge. 


\begin{tabular}{lccc}
\hline & CEM & CHA & WHA \\
\hline Coverage $(|\eta|)$ & $0-1.1$ & $0-0.9$ & $0.7-1.3$ \\
Tower Size $(\Delta \eta \times \Delta \phi)$ & $0.1 \times 15^{\circ}$ & $0.1 \times 15^{\circ}$ & $0.1 \times 15^{\circ}$ \\
Module Length & $250 \mathrm{~cm}$ & $250 \mathrm{~cm}$ & $100 \mathrm{~cm}$ \\
Module Width & $15^{\circ}$ & $15^{\circ}$ & $80 \mathrm{~cm}$ \\
Number of Modules & 48 & 48 & 48 \\
Active Medium & polystyrene & acrylic & acrylic \\
& scintillator & scintillator & scintillator \\
Thickness & $5 \mathrm{~mm}$ & $10 \mathrm{~mm}$ & $10 \mathrm{~mm}$ \\
Number of Layers & 31 & 32 & 15 \\
Absorber & $\mathrm{Pb}$ & $\mathrm{Fe}$ & Fe \\
Thickness & $3 \mathrm{~mm}$ & $25 \mathrm{~mm}$ & $51 \mathrm{~mm}$ \\
Number of Layers & 30 & 32 & 15 \\
Energy Resolution & & & $75 \% / \sqrt{E_{T}} \oplus 4 \%$ \\
$(\sigma(E) / E(G e V))$ & $13.7 \% / \sqrt{E_{T}} \oplus 2 \%$ & $50 \% / \sqrt{E_{T}} \oplus 3 \%$ &
\end{tabular}

Table 2.3: The physical properties for the central and endwall calorimeters. In the last row, the symbol $\oplus$ indicates that the constant term is to be added in quadrature to the resolution.

scintillator. There are 23 layers in depth for a total thickness of about 21 radiation lengths at normal incidence. The energy resolution of the PEM is approximately $16 \% / \sqrt{E} \oplus 1 \%$. Table 2.4 lists detailed characteristics of the plug calorimeters.

\subsubsection{The Muon Detectors}

Located outside of the central hadronic calorimeter is the central muon detection system. The central muon detector (CMU) consists of 4 layers of single wire drift cells. The CMU has 24 wedges corresponding to each central calorimeter wedge. Each of them is $12.6^{\circ}$ wide comprising 3 towers with a $2.4^{\circ}$ gap between each $\mathrm{CMU}$ wedge. In the center of each drift cell, a sense wire is running the length of the wedge $(226 \mathrm{~cm})$. The sense wires in the outer two layers are offset by $2 \mathrm{~mm}$ to resolve the ambiguity of track measurement in azimuth (Figure 2.8). A track is measured in 


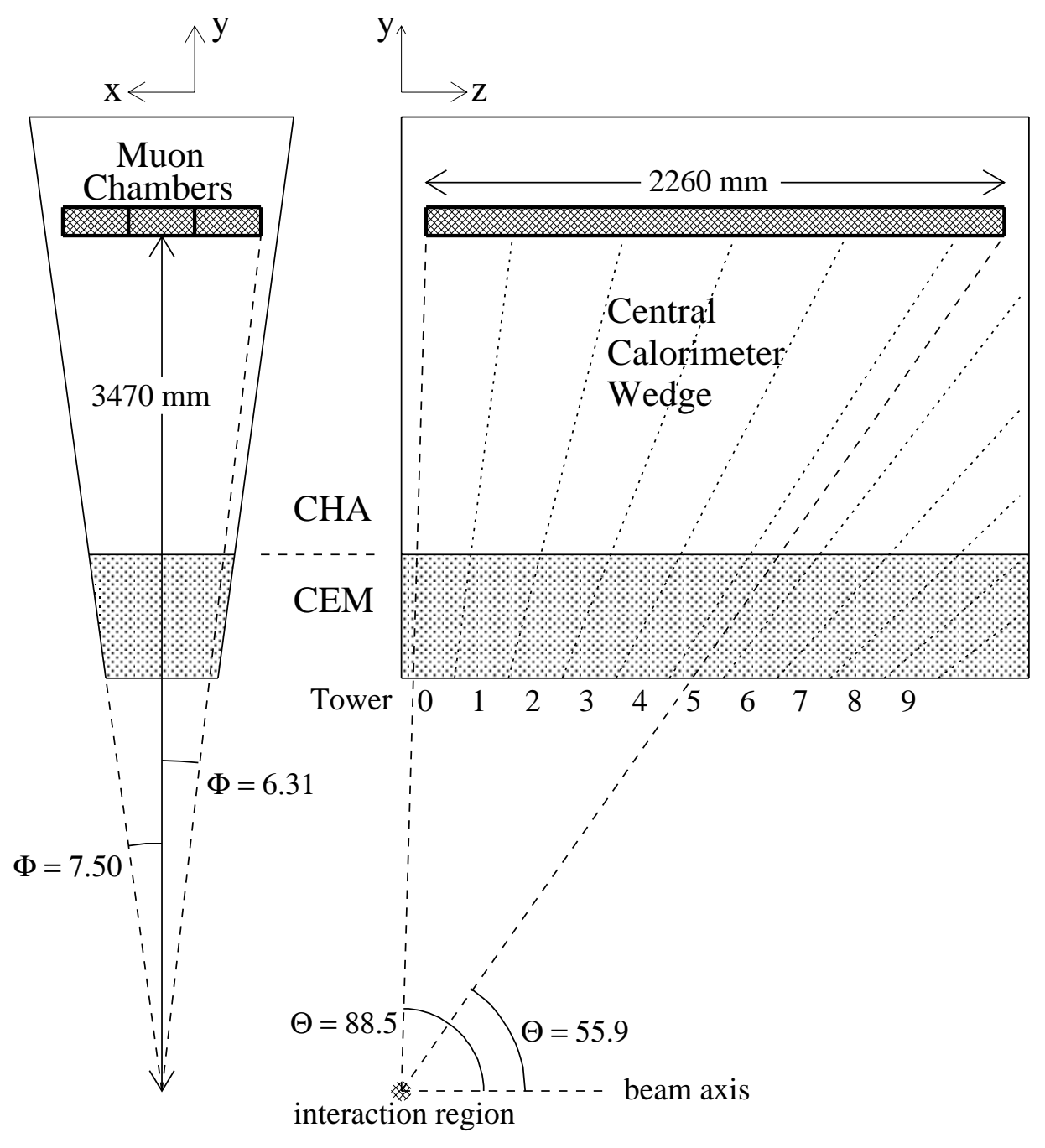

Figure 2.7: Geometry of a central calorimeter wedge and its towers. 


\begin{tabular}{lcc}
\hline \hline & EM (PEM) & Hadron (PHA) \\
\hline Segmentation & $\sim 8 \times 8 \mathrm{~cm}^{2}$ & $\sim 24 \times 24 \mathrm{~cm}^{2}$ \\
Total Channels & 960 & 864 \\
Thickness & $21 X_{0}, 1 \lambda_{0}$ & $7 \lambda_{0}$ \\
Density & $0.36 \rho_{P b}$ & $0.75 \rho_{F e}$ \\
Samples & $22+$ Preshower & 23 \\
Active medium & $4 \mathrm{~mm} \mathrm{Scint}$ & $6 \mathrm{~mm} \mathrm{Scint}$ \\
Absorber medium & $4.5 \mathrm{~mm} \mathrm{~Pb}$ & $2 \mathrm{inch} \mathrm{Fe}$ \\
Resolution & $16 \% / \sqrt{E} \oplus 1 \%$ & $80 \% / \sqrt{E} \oplus 5 \%$ \\
\hline \hline
\end{tabular}

Table 2.4: Characteristics of the plug calorimeters.

the $r-\phi$ plane with a resolution of $250 \mu \mathrm{m}$ and $1.2 \mathrm{~mm}$ in $z$ direction. Tracks are identified as muons if they leave hits in at least 3 of the 4 layers. The track segment from these layers is called a muon stub.

Additional steel absorbers are located in the outside of the CMU. Two $60 \mathrm{~cm}$ thick walls of steel stand along the side of the detector at $x= \pm \sim 540 \mathrm{~cm}$. For the top and bottom side of the detector, the steel return yokes of the solenoid at $y= \pm \sim 480 \mathrm{~cm}$ provide the additional hadron absorption. Muons passing these steel absorbers can be detected in the central muon upgrade (CMP) which comprises 4 layers of drift cells. In Run IB, there were $\phi$ gaps of the return yokes and CMP chambers in the regions $80^{\circ}<\phi<100^{\circ}$ and $260^{\circ}<\phi<280^{\circ}$. These gaps are covered with additional steel and chambers in Run II which results in a 17\% CMP coverage increase. The central muon extension(CMX) extends the pseudorapidity coverage from 0.6 to 1.0. The CMX consists of conical sections of drift tubes. In Run I, it had $30^{\circ}$ azimuthal gaps at the top of the detector and $90^{\circ}$ gaps at the 


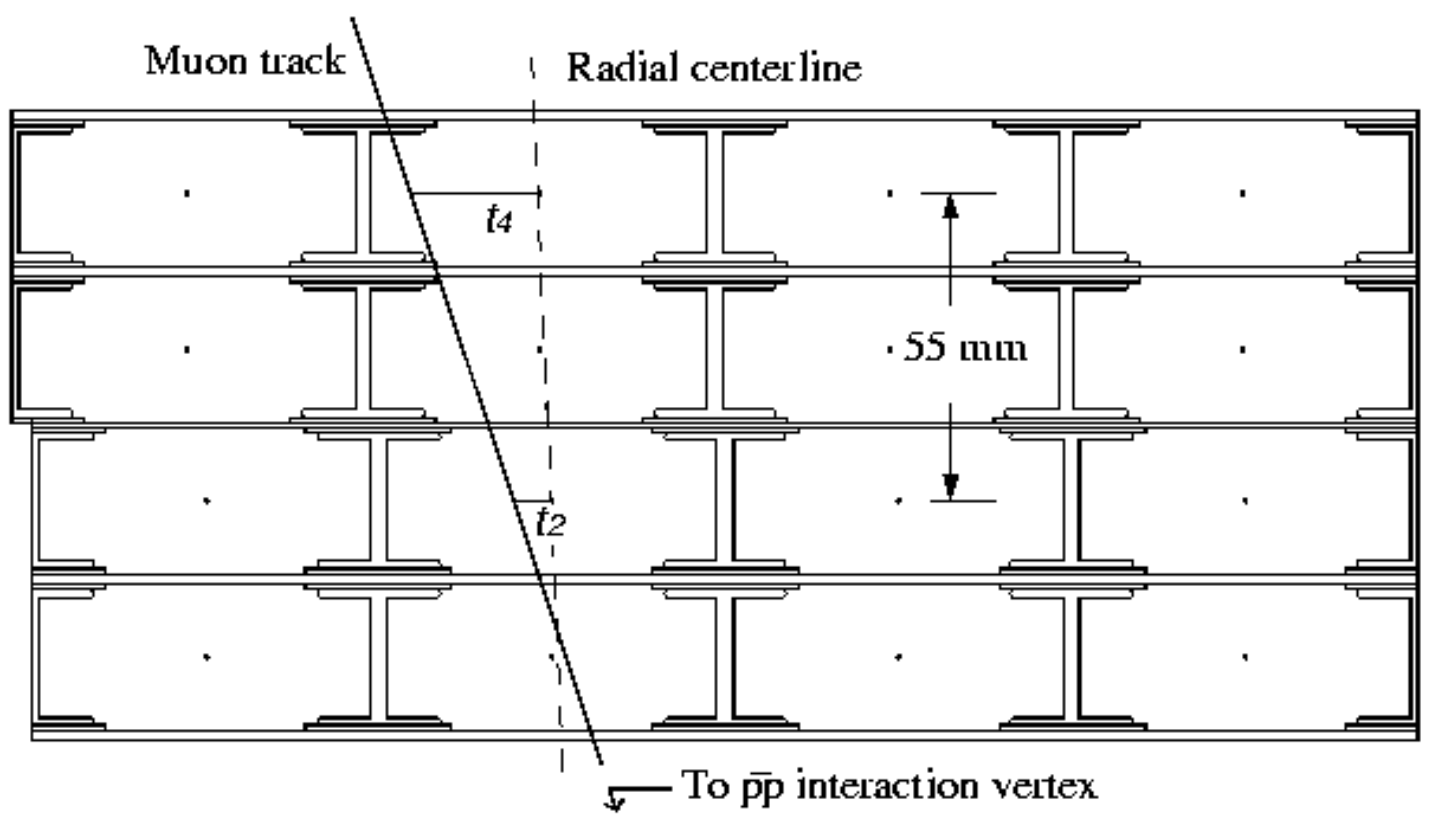

Figure 2.8: A transverse view of a muon chamber tower showing the offset of sense wire in the outer two layers. 
bottom. The gap at the top on the west side of the detector and the two gaps at the bottom (east and west) are instrumented for Run II. The coverages of CMU, CMP and CMX in $\eta, \phi$ ranges are shown in Figure 2.9.

\subsubsection{Event Triggers}

In hadron collider experiments, the trigger system plays an important role because the rate of collision far exceeds the rate of writing data to permanent storage. In Run II, the bunch crossing rate is $2.5 \mathrm{MHz}$ while the storage speed is about $50 \mathrm{~Hz}$. The CDF trigger system was completely replaced for Run II in order to accommodate the large increase of the collision rate from $280 \mathrm{kHz}$ of Run IB. The trigger system is divided into three levels which gradually reduce the rate of events to minimize the deadtime in the next level of trigger system.

The level 1 trigger uses the information from calorimeter energy clusters and charged tracks reconstructed by the track processor (XFT) [29]. The fully pipelined design of the level 1 trigger provides $5.5 \mu$ sec latency of event data and $40 \mathrm{kHz}$ of average accept rate which allows the deadtime in the level 2 trigger to be less than 10\%. The level 2 triggers which perform more sophisticated event rejection reduces the event rate to $300 \mathrm{~Hz}$. In addition to using the information from the level 1 trigger, the level 2 trigger uses more detailed information from the calorimeters and information from the SVX. The level 3 trigger is a software trigger which reconstructs physics objects such as leptons, jets and $\mathscr{E}_{T}$. The event data are filtered using the

reconstructed information and recorded into the permanent storage at the rate of $50 \mathrm{~Hz}$. 


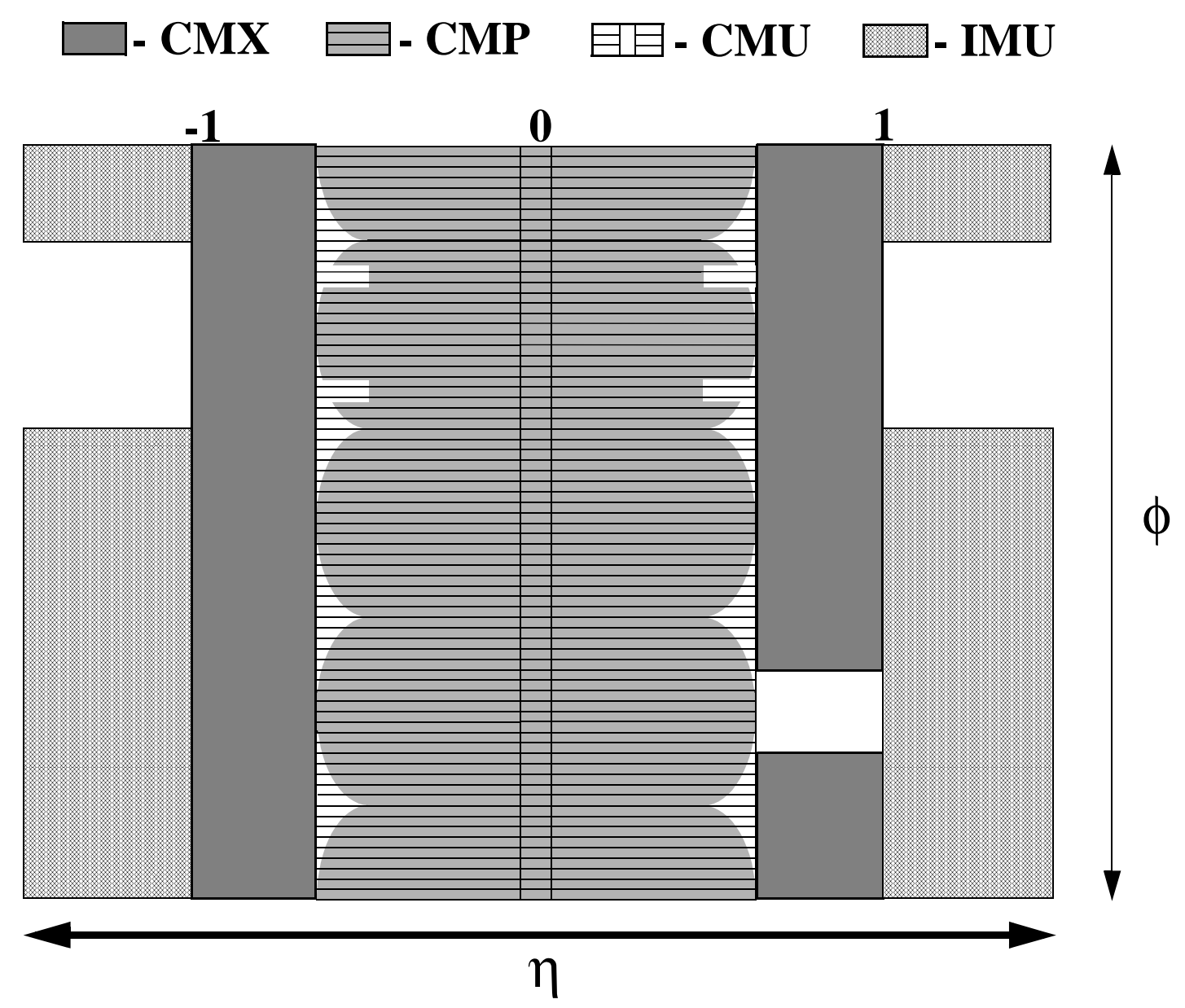

Figure 2.9: The coverage of CDF muon detectors in azimuth $\phi$ and pseudorapidity $\eta$. 


\section{CHAPTER 3}

\section{EVENT SELECTION AND ACCEPTANCE OF $t \bar{t}$ EVENT}

The cross section measurement in this thesis has been performed using the dilepton decay channel in the $t \bar{t}$ pair production. As discussed in Chapter 1, the signature of this channel is two highly energetic leptons, large missing energy and two hadronic jets from $b$ decays (Figure 1.2). Despite its relatively small branching fraction among the total $t \bar{t}$ decay channels, the dilepton channel has a big advantage in that it clearly separates the $t \bar{t}$ signal from the backgrounds. This is due primarily to the ease of identifying two high momentum charged leptons (electrons or muons). Since the lepton + jets channel of the $t \bar{t}$ decay depends largely on the measurement and identification of jets, it necessarily involves a large uncertainty from the measurement of jets. Also it has difficulties separating the signal from a large background of $W+$ jets production in the $p \bar{p}$ collision.

There are several kinds of background processes for the $t \bar{t}$ dilepton channel within the Standard Model. The largest sources of the backgrounds include Drell-Yan (Figure 4.1), $Z^{0} \rightarrow \tau \tau$ and di-boson production (Figure 4.8). The two jets involved in the $t \bar{t}$ dilepton channel provide a way to discriminate the backgrounds from the

$t \bar{t}$ signal. As will be discussed in Chapter 4, most of the tree level processes of the 
backgrounds do not involve jets. So the requirement of 2 or more jets in the event strongly separates the $t \bar{t}$ production from other Standard Model processes involving two leptons.

The existence of the missing energy in the $t \bar{t}$ events is also a feature which contrasts with other Standard Model backgrounds. The Drell-Yan and $Z^{0} \rightarrow \tau \tau$ processes that will be discussed in Chapter 4 show generally a small missing energy in the events. This is another useful handle for the background separation.

The selection procedure for the candidate events therefore begins by looking for the events with high $p_{T}$ electrons or muons. The leptons from the $W$ decays generally have a large transverse momentum as shown in Figures 3.1 and 3.2. The requirement of high $p_{T}$ leptons distinguishes leptons from other decay processes such as a semileptonic $b$ decay, which produces a lower $p_{T}$ lepton. There are established procedures for electron and muon identification using the observed quantities from the detector. These identification criteria are devised to keep maximum amount of real leptons but to remove hadronic objects mimicking leptons.

The total detection efficiency of $t \bar{t}$ events is affected by the geometric limitation of the detector, the kinematic requirement of high $p_{T}$ leptons and the lepton identification. Furthermore, there are additional selection criteria to separate the dilepton events of $t \bar{t}$ production from other Standard Model processes. These are the requirements on the missing energy, invariant mass of the two leptons, two jets and the total transverse energy of the events. These requirements are designed to 

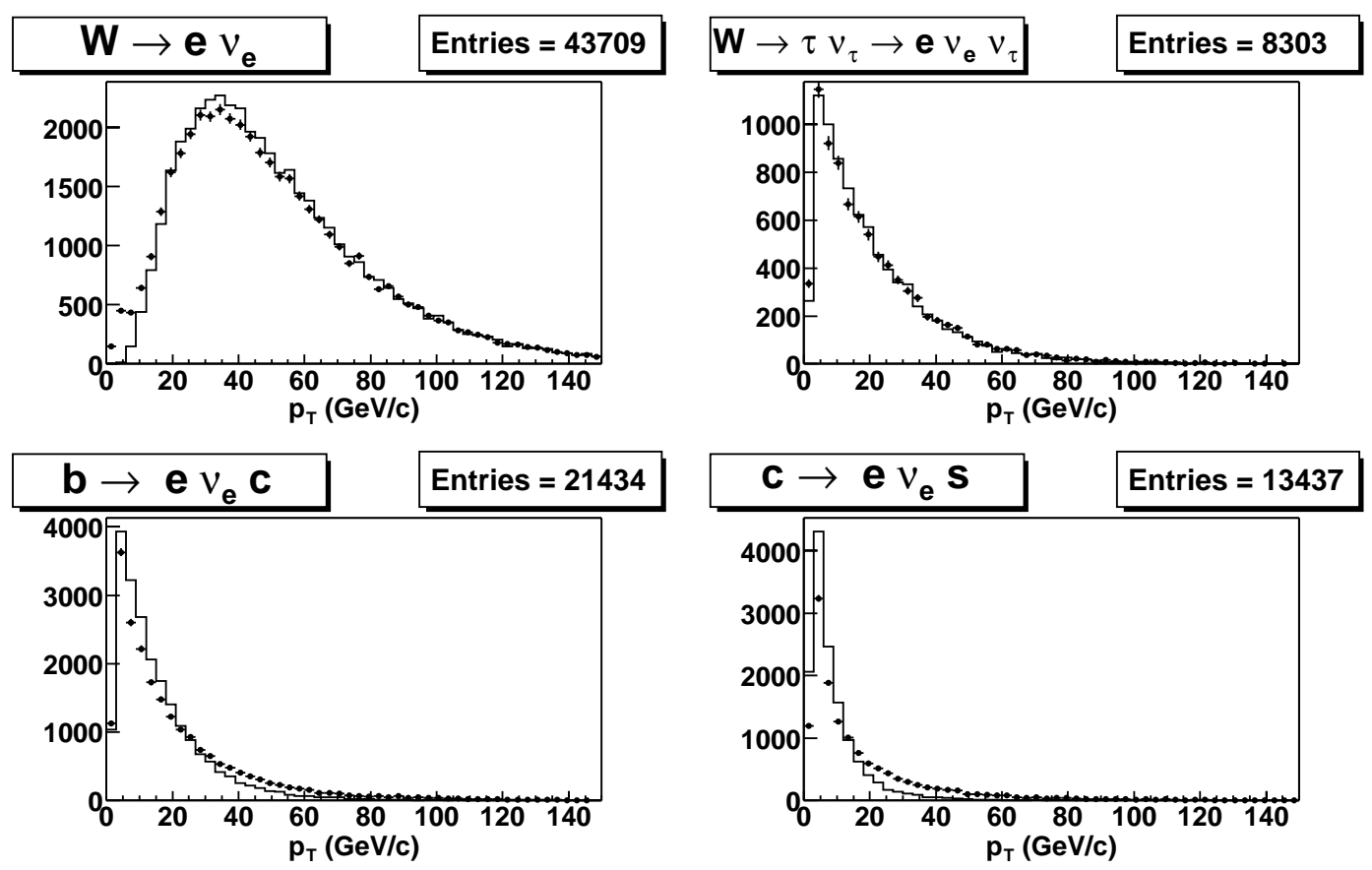

Figure 3.1: Electron $E_{T}$ of generator level (histogram) and reconstructed level (points) for the PYTHIA $t \bar{t}$ Monte Carlo sample with $m_{t}=175 \mathrm{GeV} / \mathrm{c}^{2} . W \rightarrow e \nu_{e}$ (top left), $W \rightarrow \tau \nu_{\tau} \rightarrow e \nu_{e} \nu_{\tau}$ (top right), $b \rightarrow e \nu_{e} c$ (bottom left), $c \rightarrow e \nu_{e} s$ (bottom right). 

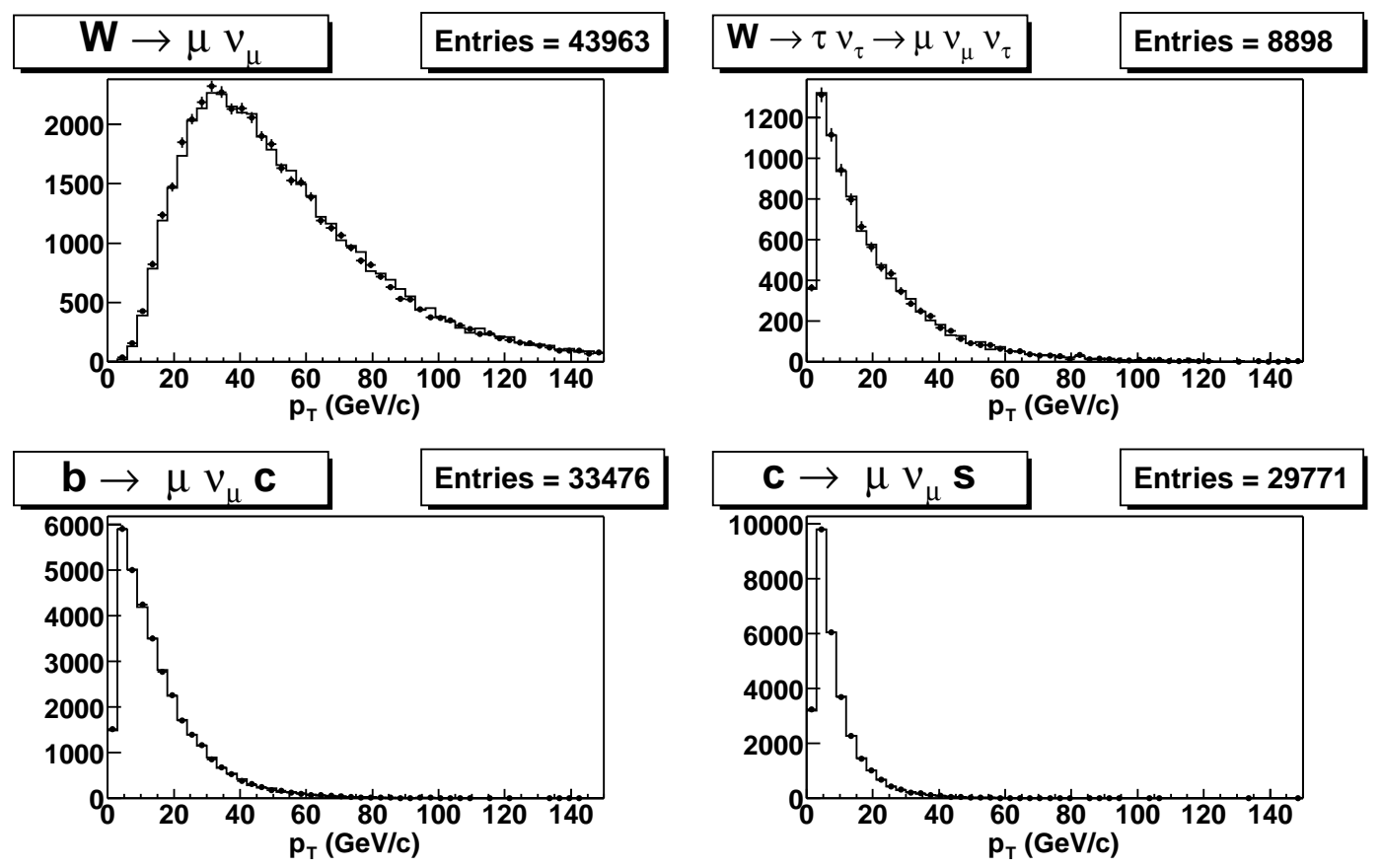

Figure 3.2: Muon $E_{T}$ of generator level (histogram) and reconstructed level (points) for the PYTHIA $t \bar{t}$ Monte Carlo sample with $m_{t}=175 \mathrm{GeV} / \mathrm{c}^{2} . W \rightarrow \mu \nu_{\mu}$ (top left), $W \rightarrow \tau \nu_{\tau} \rightarrow \mu \nu_{\mu} \nu_{\tau}$ (top right), $b \rightarrow \mu \nu_{\mu} c$ (bottom left), $c \rightarrow \mu \nu_{\mu} s$ (bottom right). 
further reduce the backgrounds from Drell-Yan, $Z^{0} \rightarrow \tau \tau$ and di-boson production. The overall efficiency including these additional selections constitutes the $t \bar{t}$ total acceptance $\epsilon_{d i l}$ that will be discussed in Section 3.4.

\subsection{Electron Selection}

Electrons deposit most of their energy in a few electromagnetic calorimeter towers. In addition, an electron will leave a track in the COT and a small shower in the CES. The information from these detector components is exploited in the electron identification. The electrons in the central region $(|\eta|<1.1)$ and plug region $(1.2<|\eta|<2.8)$ with $E_{T}>20 \mathrm{GeV}$ are considered. They have different identification variables because the detector characteristics are different. In the plug region, charge identification is difficult because there is no track reconstruction by the COT. The majority of the plug electrons do not have track momentum or charge information. However, the silicon detector can reconstruct the track segments in the high $\eta$ region which is not covered by the COT. The phoenix tracking algorithm takes advantage of the silicon track segments providing charge and momentum information for the plug electron [30]. Three classes of electrons are defined: the tight central electron (TCE), the phoenix electron (PHX) and the plug electron (PEM). The PEM contains only those plug electrons in which the phoenix algorithm fails to reconstruct the track.

In order to improve the purity, electrons are restricted to a fiducial region where the energy of the electrons are well measured. For the CEM, in the transverse plane, the CES shower position must be at least $3.2 \mathrm{~cm}$ from a CEM wedge boundary in $\phi$. The width of a CEM wedge at the CES radius is $48.5 \mathrm{~cm}$. The cluster in the 
$z$ direction must be at least $9 \mathrm{~cm}$ from the transverse plane at $z=0$ to avoid the gap between the two cylindrical halves of the central calorimeters. The seed tower of the cluster must not be tower 9 which is at the largest pseudo-rapidity (Figure 2.7). The seed tower of the cluster must not be in tower 7 of the wedge including the chimney module where tower 7, 8, 9 are missing due to cryogenic service lines to the superconducting solenoid. For the plug region, the PES cluster of the electron must be in $1.2<|\eta|<2.8$.

\subsubsection{Electron Identification Requirements}

The following variables are used to identify the central electrons (CEM).

- Transverse energy $\left(E_{T}\right)$ : This is measured in the electromagnetic calorimeter. The energy is corrected for variations in tower-to-tower responses.

- Track transverse momentum $\left(p_{T}\right)$ : The transverse momentum of the track is obtained from the track curvature measured in the COT. The $p_{T}$ is refitted with the constraint that it pass through the beam position. This improves the resolution of $p_{T}$.

- $E_{H A D} / E_{E M}$ : This is the ratio of the hadronic calorimeter energy to the electromagnetic calorimeter energy for the cluster.

- Lshr: This variable compares the observed sharing of energy deposition between towers to that expected for a true electromagnetic shower. It is defined as

$$
L s h r=0.14 \frac{\sum_{i}\left(E_{i}^{o b s}-E_{i}^{e x p}\right)}{\sqrt{(0.14 \sqrt{E})^{2}+\sum_{i} \sigma_{E_{i}^{e x p}}^{2}}}
$$


where the sums are over the towers in the EM cluster adjacent to the seed tower and in the same wedge as the seed tower. $E_{i}^{o b s}$ is the measured energy in an adjacent tower, $E_{i}^{e x p}$ is the predicted energy deposition in the adjacent tower from test beam electrons. $\sigma_{E_{i}^{e x p}}$ is the uncertainty in $E_{i}^{e x p}$. The CEM calorimeter energy resolution of the cluster provides the term of $0.14 \sqrt{E}$.

- $Q \times \Delta x$ : The quantity $\Delta x$ represents the distance in the $r-\phi$ plane between the extrapolated track position and the CES maximum shower position. This is multiplied by the electric charge $(Q)$ of the matched track.

- $|\Delta z|$ : This quantity represents the distance in the $r-z$ plane between the extrapolated track position and the CES maximum shower position.

- $\chi_{\text {Strip }}^{2}$ : This is the $\chi^{2}$ resulting from the comparison of the CES shower profile in the $z$ view between the electron candidate and test beam electrons.

- $E / P$ : This is the ratio of the electromagnetic calorimeter energy to the track momentum.

- $N_{\text {Axial }}$ : This is the number of axial COT superlayers with at least 7 hits associated with the track.

- $N_{\text {Stereo }}$ : This is the number of stereo COT superlayers with at least 7 hits associated with the track.

- $z_{0}$ : This is the distance along the beam axis between the origin and the reconstructed track.

In addition to the cuts on the variables described above, an algorithm is used to remove the electrons from photon conversions. 
For the plug electron selection, different variables are used. $\chi_{3 \times 3}^{2}$ is a quantitative comparison of the pattern of EM energy deposition between a given cluster and the test beam result. The pesProfileRatio5by 9 is a simple ratio of strip energies in the PES cluster defined as

$$
\text { pesProfileRatio5by } 9=\frac{\Sigma \text { Energy of central } 5 \text { strips }}{\text { Total energy of the PES cluster }} .
$$

There are two layers in the PES detector. The $\mathrm{U}$ and $\mathrm{V}$ layer have strips aligned at $+22.5^{\circ}$ and $-22.5^{\circ}$ with respect to the radial direction. The pesProfileRatio5by 9 variables are assigned for $\mathrm{U}$ and $\mathrm{V}$ layers separately. $\triangle R_{P E M-P E S}$ is the spatial distance between the PEM cluster and the PES 2d cluster defined as

$$
\Delta R_{P E M-P E S}=\sqrt{\left(\eta_{p e m 3 \times 3}-\eta_{p e s 2 d}\right)^{2}+\left(\phi_{p e m 3 \times 3}-\phi_{p e s 2 d}\right)^{2}} .
$$

In addition to the PEM identification requirements, PHX has the track quality variables to be met. The cuts used to select TCE, PHX, PEM are shown in Table 3.1, 3.2.

\subsection{Muon Selection}

Muons are identified by hits left in the muon chambers. Because muons are minimum-ionizing particles, they will only leave a small amount of energy in the calorimeters. However, muons should leave a track in the COT which can be 


\begin{tabular}{ccc}
\hline Variable & Selection criteria & \\
\hline$E_{T}$ & $>20 \mathrm{GeV}$ & beam constrained \\
$p_{T}$ & $>10 \mathrm{GeV} / \mathrm{c}$ & \\
$E / p$ & $<2.0\left(E_{T}<100 \mathrm{GeV}\right)$ & \\
& $p_{T}>50.0 \mathrm{GeV}\left(E_{T} \geq 100 \mathrm{GeV}\right)$ & \\
$E_{H A D} / E_{E M}$ & $<0.055+0.00045 \times E$ & \\
$L s h r$ & $<0.2$ & \\
$Q \times \Delta x$ & $>3.0,<1.5 \mathrm{~cm}$ & \\
$|\Delta z|$ & $<3.0 \mathrm{~cm}$ & \\
$\chi_{\text {strip }}^{2}$ & $\geq 10.0$ & at least 7 hits in a SL \\
$N_{\text {Axial }}$ & $\geq 3$ & \\
$N_{\text {Stereo }}$ & $<60 \mathrm{~cm}$ & \\
$\left|z_{0}\right|$ & & \\
\hline
\end{tabular}

Table 3.1: Selection cuts for TCE identification variables.

\begin{tabular}{ccc}
\hline Variable & Selection criteria & \\
\hline \hline$E_{T}$ & $>20 \mathrm{GeV}$ & \\
$E_{H A D} / E_{E M}$ & $<0.05$ & $(E \leq 100 \mathrm{GeV})$ \\
$\chi_{3 \times 3}^{2}$ & $<0.05+0.026 \times \log (E / 100 \mathrm{GeV})$ & $(E>100 \mathrm{GeV})$ \\
$\Delta R_{P E M-P E S}$ & $<10.0$ & \\
pesProfileRatio5by9(U) & $<3.0 \mathrm{~cm}$ & \\
pesProfileRatio5by9 $(\mathrm{V})$ & $>0.65$ & Silicon hits \\
$N_{\text {SiHit }}$ & $>0.65$ & in the Phoenix track \\
& $\geq 4$ & $z_{0}$ of the Phoenix track \\
\hline$z_{0} \mid$ & $<60 \mathrm{~cm}$ &
\end{tabular}

Table 3.2: Selection cuts for PEM and PHX identification variables. 
matched to the hits in the muon chamber. There are four classes of muons defined by the muon chambers through which they pass. The CMU and CMP muon classes are contained solely in those chambers. The CMUP muon has hits in both the CMU and CMP chambers simultaneously. The CMX muon passes through the CMX chamber. An additional class of the muon is the minimum ionizing particle $(\mathrm{CMIO})$ which is a track without hits in any muon chamber but with minimal energy deposition in the calorimeter. They are only considered if they point to a non-fiducial region of the muon system.

\subsubsection{Muon Identification Requirements}

The following variables are used to identify the central muons and the minimum ionizing particle.

- $P_{T}$ : This is the transverse momentum of the track obtained from the track curvature measured in the COT. The $p_{T}$ is refitted with the constraint that it pass through the beam position, which improves the resolution of $p_{T}$.

- $E_{E M}, E_{H A D}$ : These are the energies deposited in the electromagnetic and hadronic calorimeter respectively.

- $|\Delta x|$ : This is the distance in the $r-\phi$ plane between the extrapolated track position and the track segment in the relevant muon chamber.

- $d 0$ : This is the impact parameter of the muon track. This is the distance of the closest approach between the reconstructed muon track and the beam axis in the $r-\phi$ plane. This removes muons from cosmic rays which do not originate from the primary interaction near the beam axis. 


\begin{tabular}{ccc}
\hline Variable & Selection criteria & \\
\hline \hline$p_{T}$ & $>20 \mathrm{GeV} / \mathrm{c}$ & beam constrained \\
$E_{E M}$ & $<2.0 \mathrm{GeV}$ & \\
$E_{E M}(p>100 \mathrm{GeV} / \mathrm{c})$ & $<2.0+0.0115 \times(p-100) \mathrm{GeV}$ & \\
$E_{H A D}$ & $<6.0 \mathrm{GeV}$ & \\
$E_{H A D}(p>100 \mathrm{GeV} / c)$ & $<6.0+0.028 \times(p-100) \mathrm{GeV}$ & CMIO only \\
$E_{E M}+E_{H A D}$ & $>0.1 \mathrm{GeV}$ & without silicon hits \\
$d_{0}$ & $<0.2 \mathrm{~cm}$ & with silicon hits \\
& $<0.02 \mathrm{~cm}$ & CMU, CMUP \\
$|\Delta x|_{\text {CMU }}$ & $<3.0 \mathrm{~cm}$ & CMP, CMUP \\
$|\Delta x|_{C M P}$ & $<5.0 \mathrm{~cm}$ & CMX \\
$|\Delta x|_{\text {CMX }}$ & $<6.0 \mathrm{~cm}$ & $|\Delta x|$ is not applicable in CMIO \\
$N_{\text {Axial }}$ & $\geq 3$ & at least 7 hits in a SL \\
$N_{\text {Stereo }}$ & $\geq 3$ & at least 7 hits in a SL \\
$\left|z_{0}\right|$ & $<60 \mathrm{~cm}$ & \\
\hline
\end{tabular}

Table 3.3: Selection cuts for the central muon and minimum ionizing particle identification variables.

Similar to electron identification, the track quality variables $N_{\text {Axial }}, N_{\text {Stereo }}$ and $z_{0}$ are required for the muon tracks. The cuts used to select muons are shown in Table 3.3. The cosmic ray events may look like di-muon events if they pass close to the beam axis. The cosmic filter [32] is applied in addition to the muon identification in the event selection in the data sample. However, it is not applied to the Monte Carlo sample for the acceptance estimation because it removes $t \bar{t}$ events unnecessarily. 


\subsection{Dilepton Selection}

Selection of dilepton events starts by requiring that the event has two leptons that satisfy the requirements in the previous sections. The leptons are classified as tight and loose. The tight leptons include TCE, PHX, CMUP, CMX which have stronger lepton identification requirements. The dilepton events must have at least one tight lepton. The PEM, CMP, CMU, CMIO are considered as loose. The possible dilepton types are tight - tight, tight - loose. Therefore, a total of 26 types of lepton pairs are possible. The possible combinations of the dilepton events are shown in Table 3.4.

\subsubsection{Isolation}

Because the leptons from $W$ decays are usually more isolated than the leptons from semileptonic $b$ decays, a cut on the lepton isolation is useful for the $t \bar{t}$ dilepton selection. The lepton isolation is defined as the ratio of the calorimeter transverse energy surrounding the lepton to the calorimeter transverse energy of the lepton. It is defined as

$$
I s o 4=\frac{E_{T}^{04}}{E_{T}}
$$

where $E_{T}^{04}$ is the calorimeter transverse energy except the energy of the lepton inside a cone of radius $\Delta R=\sqrt{(\Delta \eta)^{2}+(\Delta \phi)^{2}}=0.4$ with axis as the lepton direction. $E_{T}$ is the transverse energy of the lepton. $E_{T}$ is replaced with $p_{T}$ for the muon isolation. The isolation variable can be spoiled if there is a significant leakage of 


\begin{tabular}{c|c}
\hline & Dilepton Category \\
\hline \hline \multirow{5}{*}{$e-e$} & TCE - TCE \\
& TCE - PHX \\
& TCE - PEM \\
& PHX - PHX \\
& PHX - PEM \\
\hline \multirow{5}{*}{$\mu-\mu$} & CMUP - CMUP \\
& CMUP - CMU \\
& CMUP - CMP \\
& CMUP - CMX \\
& CMUP - CMIO \\
& CMX - CMU \\
& CMX - CMP \\
& CMX - CMX \\
& CMX - CMIO \\
\hline \multirow{5}{*}{$=($ TCE - CMUP } \\
& PHX - CMUP \\
& PEM - CMUP \\
& TCE - CMU \\
& PHX - CMU \\
& TCE - CMP \\
& PHX - CMP \\
& TCE - CMX \\
& PHX - CMX \\
& PEM - CMX \\
& TCE - CMIO \\
& PHX - CMIO \\
\hline
\end{tabular}

Table 3.4: Dilepton categories for $e e, \mu \mu, e \mu$. 
energy resulting from the electrons near the edge of the cluster. A correction for this leakage is applied to the isolation variable [33]. Figures 3.3 and 3.4 show the isolation characteristics of the leptons from various sources. An isolation requirement of

$$
I s o 4<0.1
$$

is used for both leptons in the dilepton selection.

\subsubsection{Invariant Mass}

The dominant background after the lepton identification and isolation requirement in di-electron or di-muon events is the Drell-Yan process from $Z^{0}$ decay. The events from this background have a large peak in the distribution of the invariant mass of two leptons as shown in Figure 3.5. The events are rejected if the invariant mass of two leptons is in the range, $76 \mathrm{GeV}<M_{\ell \ell}<106 \mathrm{GeV}$. In order to calculate the invariant mass, the calorimeter energy is used for the vector components of electron momentum while the track momentum is used for the vector components of muon momentum. While the invariant mass cut removes a majority of events from the Drell-Yan process, only a small fraction of $t \bar{t}$ events is affected by this cut as shown in Figure 3.6. The invariant mass cut is not applied to the events of $e \mu$ because these should not originate from the Drell-Yan process.

\subsubsection{Missing $E_{T}$}

The unobserved neutrinos result in a large missing transverse energy $\left(\mathbb{E}_{T}\right)$ in the $t \bar{t}$ events. The raw $\not_{T}$ is the negative of the vector sum of the transverse energies in the calorimeter. However, the missing $E_{T}$ caused by true neutrinos should be the 

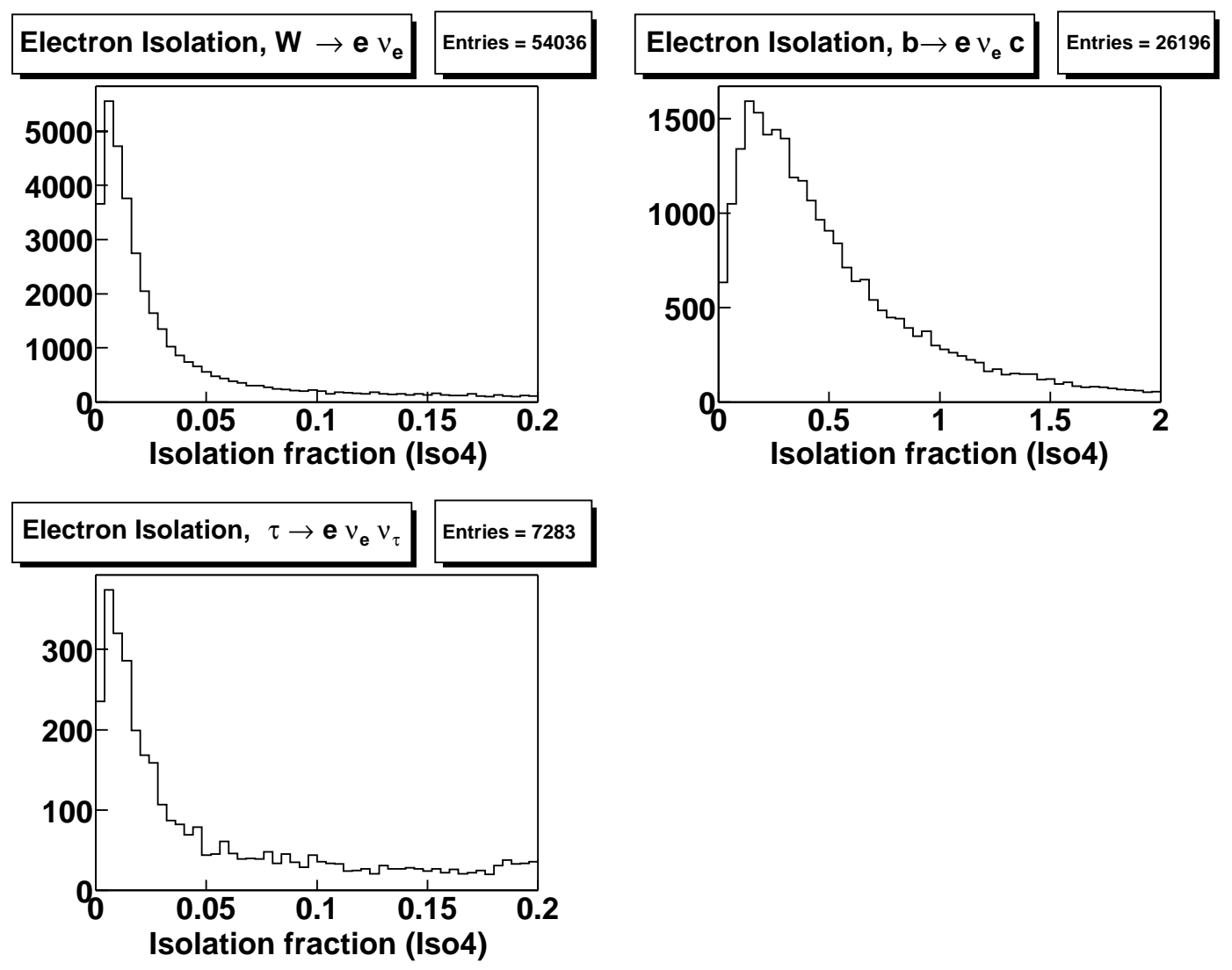

Figure 3.3: Isolation of electrons from different sources in the $t \bar{t}$ PYTHIA Monte Carlo sample with $m_{t}=175 \mathrm{GeV} / \mathrm{c}^{2}$. W $\rightarrow e \nu_{e}$ (top left), $b \rightarrow e \nu_{e} c$ (top right), $W \rightarrow \tau \nu_{\tau} \rightarrow e \nu_{e} \nu_{\tau}$ (bottom left). Note scale change of the x-axis for $b \rightarrow e \nu_{e} c$. 

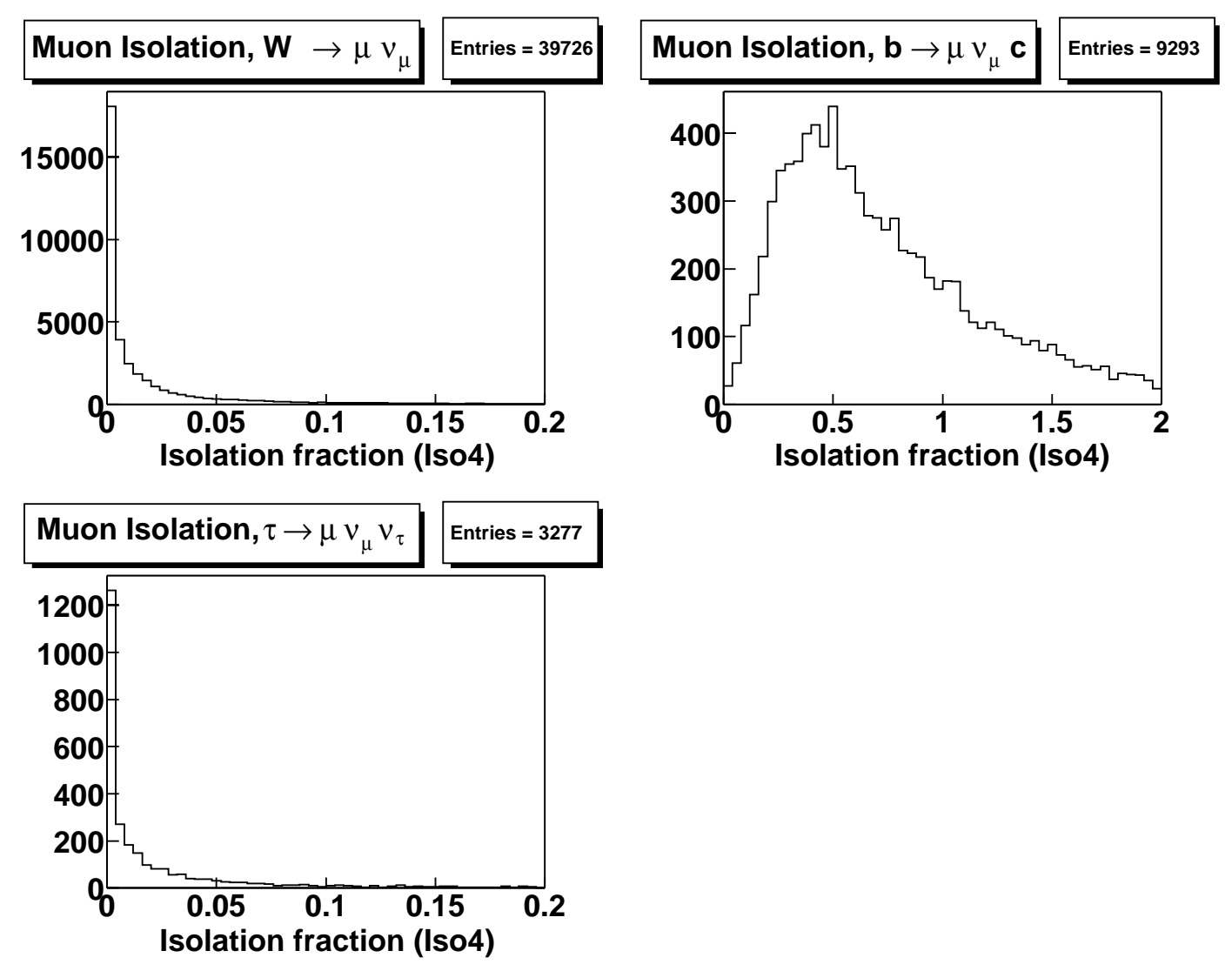

Figure 3.4: Isolation of muons from different sources in the $t \bar{t}$ PYTHIA Monte Carlo sample with $m_{t}=175 \mathrm{GeV} / \mathrm{c}^{2}$. $W \rightarrow \mu \nu_{\mu}$ (top left), $b \rightarrow \mu \nu_{\mu} c$ (top right), $W \rightarrow \tau \nu_{\tau} \rightarrow \mu \nu_{\mu} \nu_{\tau}$ (bottom left). Note scale change of the x-axis for $b \rightarrow \mu \nu_{\mu} c$. 


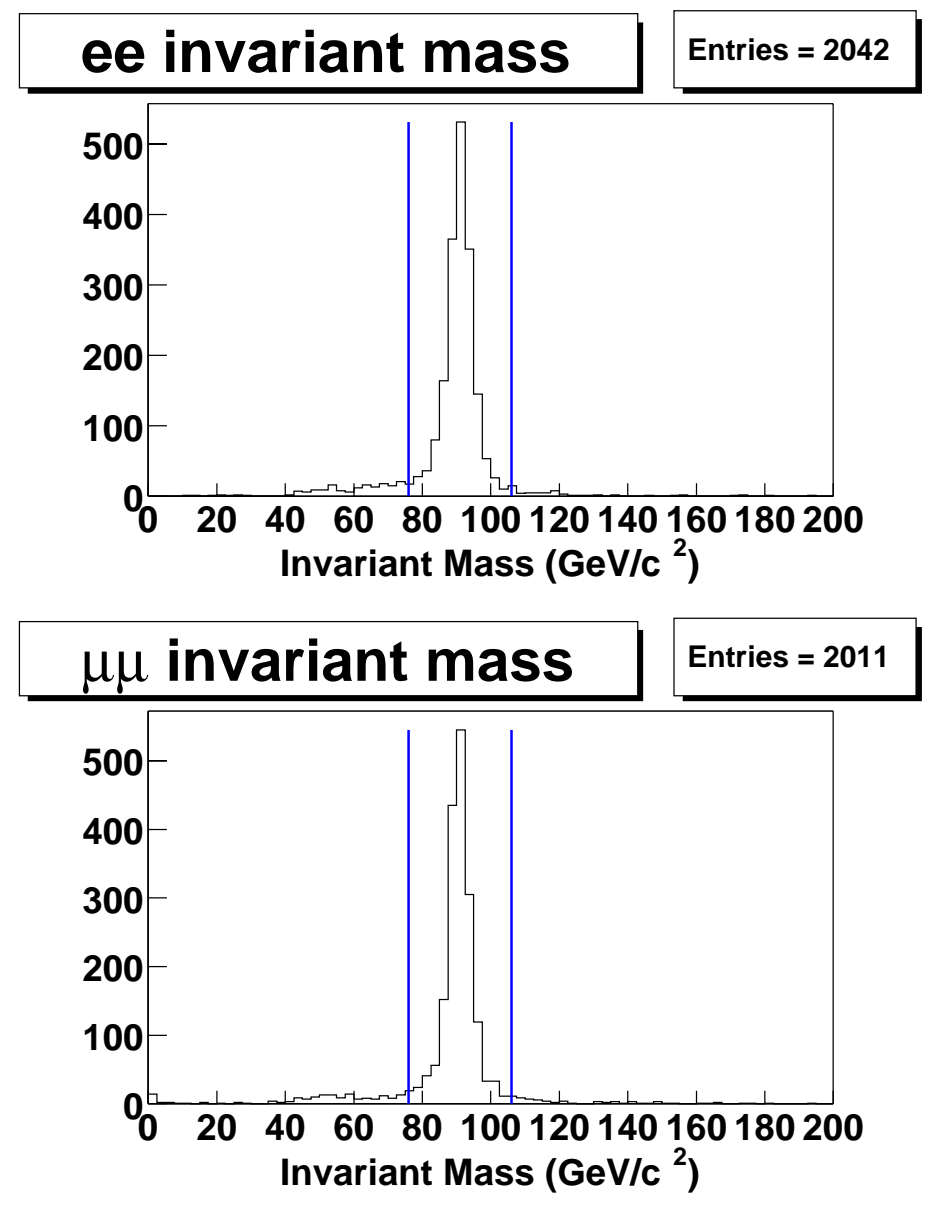

Figure 3.5: Invariant mass of electron pairs (top) and muon pairs (bottom) in the dilepton events in $109 \mathrm{pb}^{-1}$ of data. 

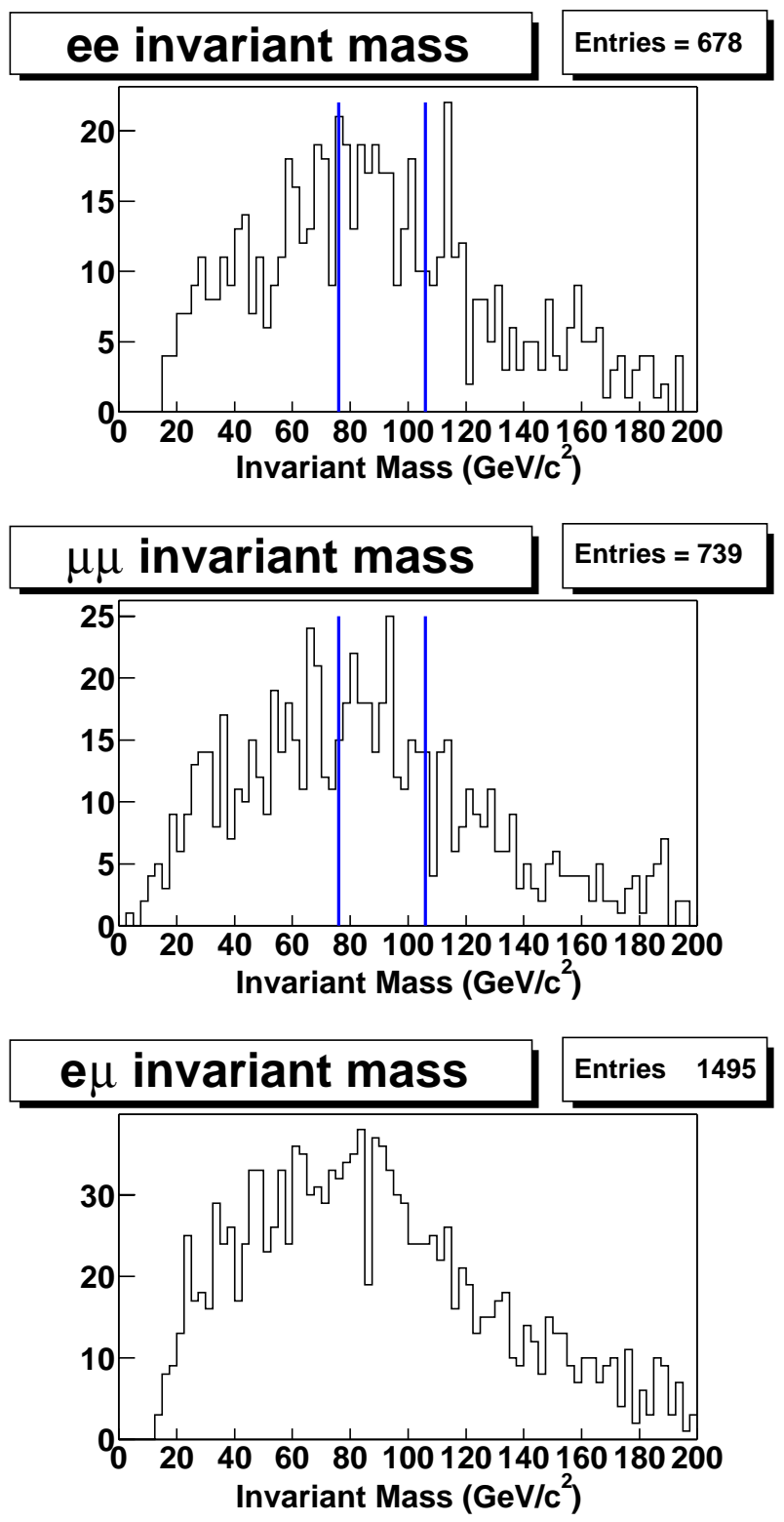

Figure 3.6: Invariant mass of the two leptons $\left(m_{\ell \ell}\right)$ in the dilepton events after the isolation requirement is imposed. Di-electron (ee, top), di-muon ( $\mu \mu$, middle), electron-muon ( $e \mu$, bottom) events in the $t \bar{t}$ PYTHIA Monte Carlo sample with $m_{t}=175 \mathrm{GeV} / \mathrm{c}^{2}$. In the $e \mu$ category, the invariant mass cut is not imposed. The vertical lines in the $e e, \mu \mu$ show the invariant mass cut of $76 \mathrm{GeV} / \mathrm{c}^{2}<m_{\ell \ell}<$ $106 \mathrm{GeV} / \mathrm{c}^{2}$. 
negative of the vector sum of all transverse energies from all particles except the neutrinos. Therefore, the transverse momenta of the muons in the events must be vectorially subtracted from the raw $\mathbb{E}_{T}$ since the muons do not deposit energy in the calorimeter. In addition, the transverse energies of jets in the events are corrected for detector effects that will be discussed in the next section. The differences between the corrected jet energies and the raw jet energies are applied to the $\not_{T}$ as well.

The magnitude and the direction of the corrected $E_{T}$ are used to select the dilepton $t \bar{t}$ events. The magnitude of $\mathbb{E}_{T}$ is required to be greater than $25 \mathrm{GeV}$. This reduces most of the Drell-Yan backgrounds and a significant amount of the $Z^{0} \rightarrow \tau \tau$ as shown in Figures 4.2, 4.3, 4.4, 4.5, and 4.6, while retaining most of the $t \bar{t}$ events as shown in Figures 3.7, 3.8, and 3.9. The azimuthal angle between the $E_{T}$ and the nearest lepton or jet is another quantity used for the selection of the $t \bar{t}$ dilepton events. If $E_{T}$ is less than $50 \mathrm{GeV}, \Delta \phi\left(E_{T}, j\right.$ or $\left.\ell\right)$ is required to be greater than $20^{\circ}$. This angular requirement is justified by the tendency of the $Z^{0} \rightarrow \tau \tau$ background to have $\mathbb{E}_{T}$ directed along one of the leptons as indicated in Figures 4.4, 4.5, and 4.6.

\subsubsection{Jet Requirements}

The two jets from $b$-quark decays can distinguish the dilepton events of $t \bar{t}$ from other Standard Model dilepton events. The raw jet $E_{T}$ is the sum of the transverse energies which are deposited in the calorimeter towers inside a cone radius $R=$ $\sqrt{(\Delta \eta)^{2}+(\Delta \phi)^{2}}$ with the center at the calorimeter tower with the largest energy deposition. For this analysis a cone radius $R=0.4$ is selected. The raw jet $E_{T}$ 's 

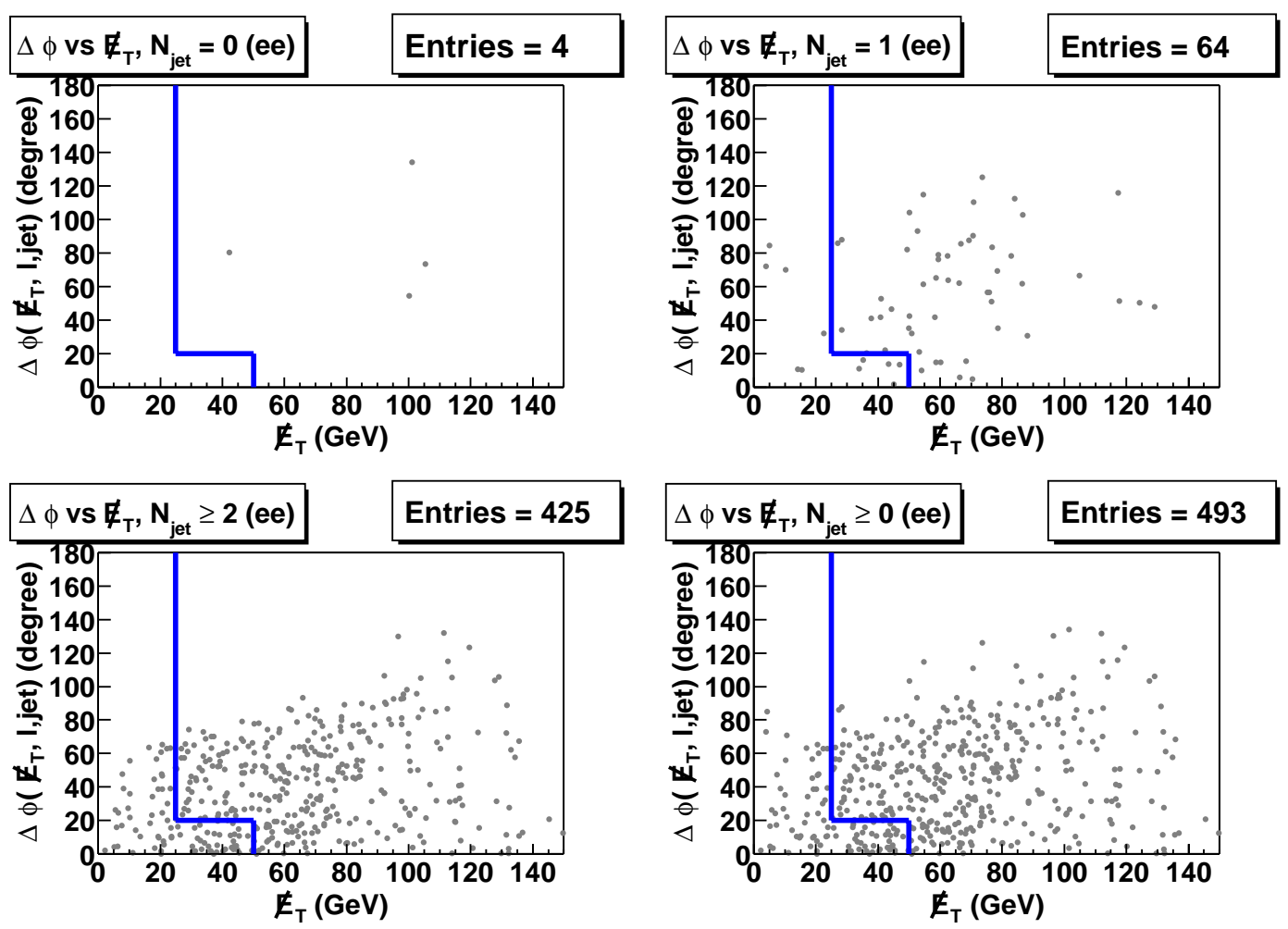

Figure 3.7: The magnitude of the missing $E_{T}\left(E_{T}, x\right.$ axis) and the $\Delta \phi$ between $Z_{T}$ and the closest jet or lepton ( $y$ axis). Di-electron events in the $t \bar{t}$ PYTHIA Monte Carlo sample with $m_{t}=175 \mathrm{GeV} / \mathrm{c}^{2}$ are shown with no jet $\left(N_{j}=0\right.$, top left), $N_{j}=1$ (top right), $N_{j} \geq 2$ (bottom left), $N_{j} \geq 0$ (bottom right). The lines show the cut imposed for the $\mathbb{E}_{T}$ requirement. 

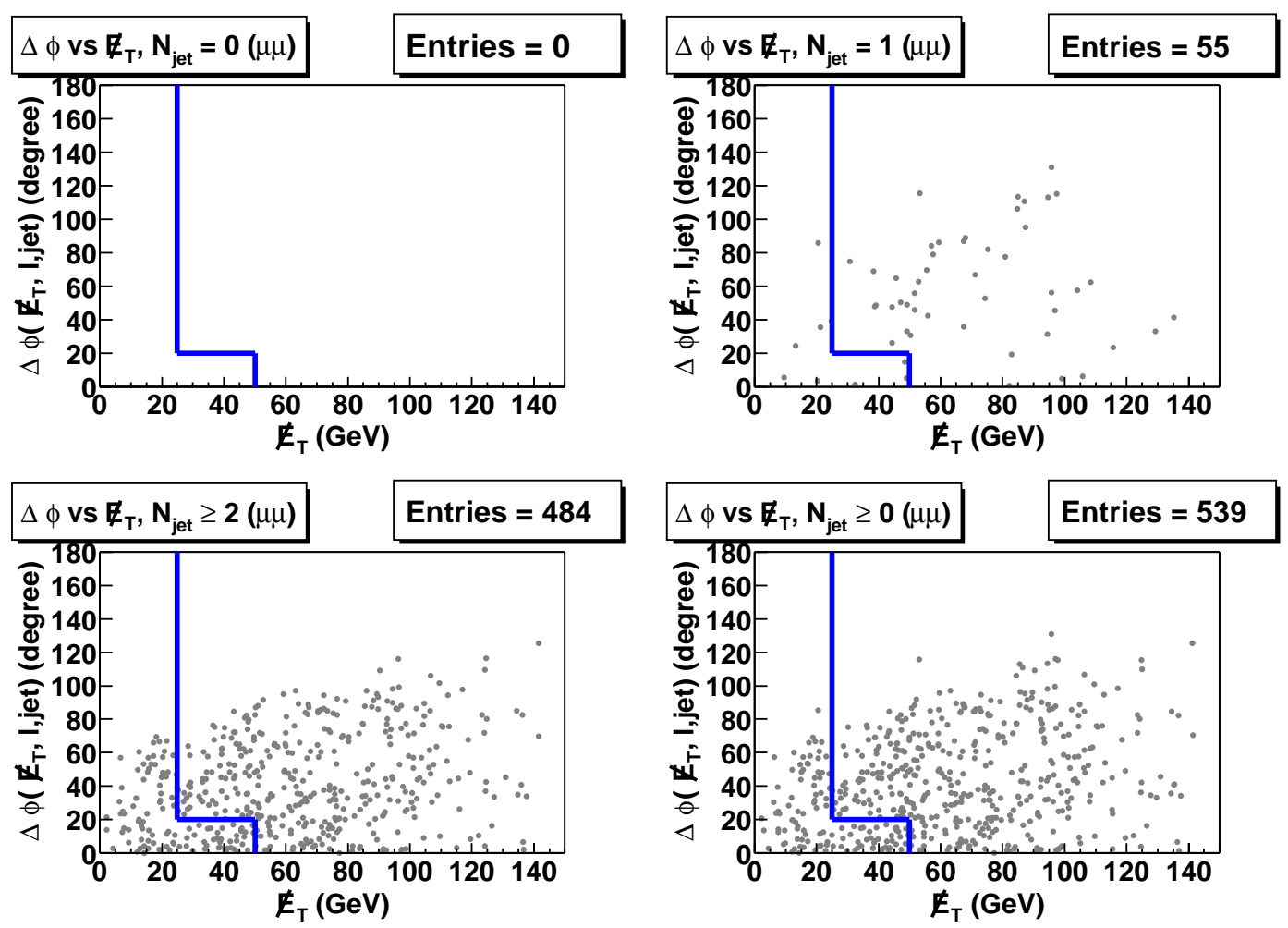

Figure 3.8: The magnitude of the missing $E_{T}\left(E_{T}, x\right.$ axis) and the $\Delta \phi$ between $\not_{T}$ and the closest jet or lepton ( $y$ axis). Di-muon events in the $t \bar{t}$ PYTHIA Monte Carlo sample with $m_{t}=175 \mathrm{GeV} / \mathrm{c}^{2}$ are shown with no jet $\left(N_{j}=0\right.$, top left), $N_{j}=1$ (top right), $N_{j} \geq 2$ (bottom left), $N_{j} \geq 0$ (bottom right). The lines show the cut imposed for the $\mathbb{E}_{T}$ requirement. 

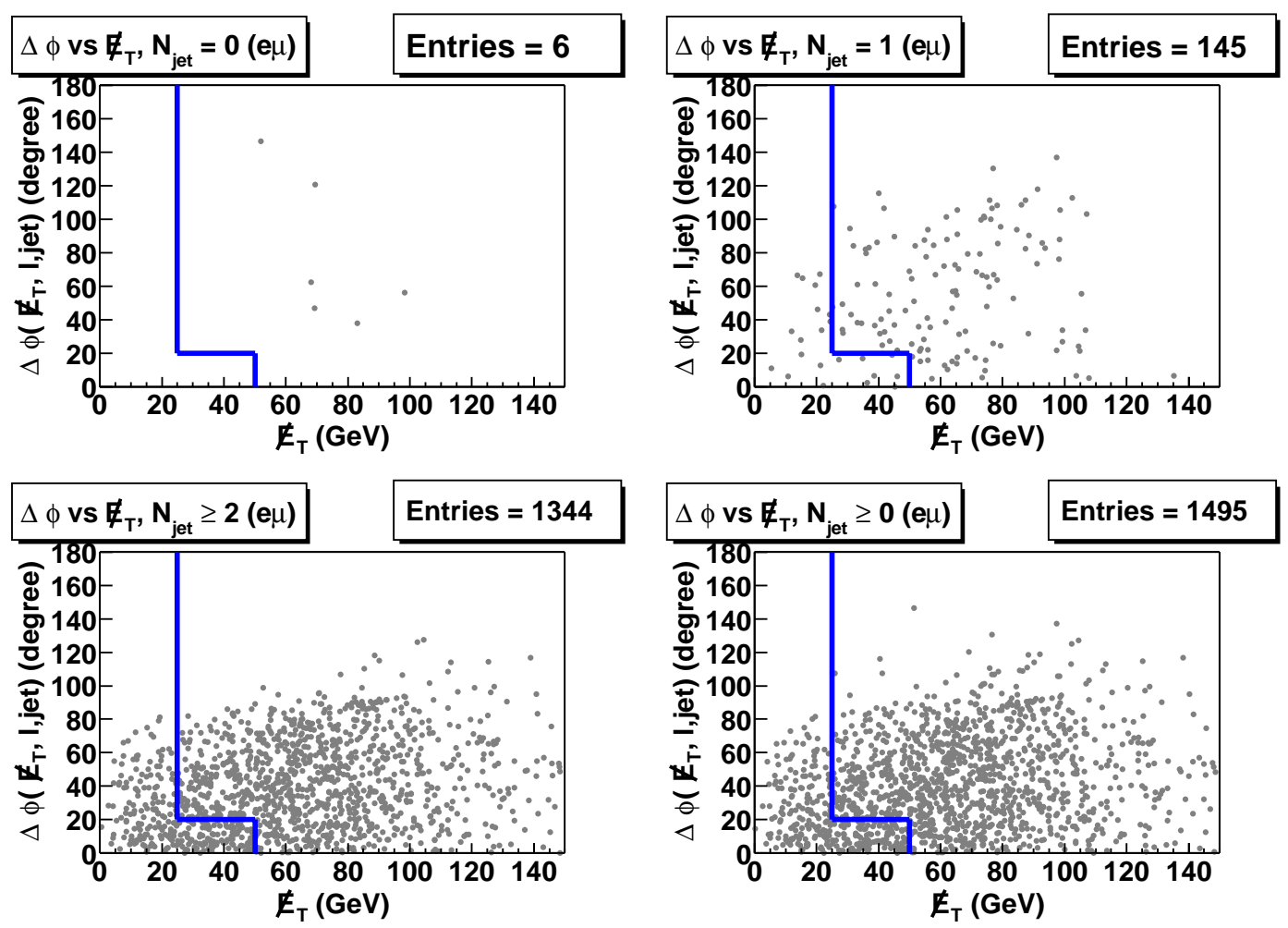

Figure 3.9: The magnitude of the missing $E_{T}\left(B_{T}, x\right.$ axis) and the $\Delta \phi$ between $\not_{T}$ and the closest jet or lepton ( $y$ axis). $e \mu$ events in the $t \bar{t}$ PYTHIA Monte Carlo sample with $m_{t}=175 \mathrm{GeV} / \mathrm{c}^{2}$ are shown with no jet $\left(N_{j}=0\right.$, top left $), N_{j}=1$ (top right), $N_{j} \geq 2$ (bottom left), $N_{j} \geq 0$ (bottom right). The lines show the cut imposed for the $\mathbb{E}_{T}$ requirement. 
are corrected for several effects. The corrected transverse energy $E_{T}^{\text {cor }}$ of a jet can be written in terms of the raw $E_{T}^{\text {raw }}$ with several correction terms as shown in Equation 3.2 [34][35].

$$
E_{T}^{c o r}(R)=\left(E_{T}^{r a w}(R) \times f_{r e l}-U E M(R)\right) \times f_{a b s}(R)-U E(R)+O C(R)
$$

The $f_{r e l}$ is the relative energy scale. It reflects relative responses of the calorimeter towers in $\eta$. The UEM is the energy resulting from the multiple interactions. The absolute energy scale $f_{a b s}$ includes various detector effects. For example, the nonuniform response in the boundary of towers and wedges is corrected in $f_{a b s}$. It also takes into account the energies not detected by calorimeter due to muons and neutrinos. The UE is the correction due to the underlying event which is associated with soft interactions between spectator partons in the collisions. The OC is the out-of-cone energy which is a fraction of energy out of the radius $R$ defined above. In this analysis, the UE and $\mathrm{OC}$ are not considered. The transverse energy inside $R \leq 0.4$ are reconstructed as a jet in this analysis.

For the jets in the dilepton events to be counted as jet candidates for selection purposes, they must not be within $\Delta R<0.4$ of any electron. This prevents the calorimeter clusters consistent with the electrons from being double counted as jets. In this analysis, jets with corrected $E_{T}>10 \mathrm{GeV}$ and $\left|\eta_{\text {det }}\right|<2.0$ are counted for selection purposes, where $\eta_{\text {det }}$ is $\eta$ measured with respect to the detector origin. The number of jets $\left(N_{j}\right)$ in an event must be greater than or equal to 2 in order for the event to be considered a $t \bar{t}$ dilepton candidate. 


\subsection{5 $H_{T}$ and Opposite Sign Requirement}

Because of the large top mass, the final state from $t \bar{t}$ production results in a large total transverse momentum. The backgrounds still remaining after the jet requirement are removed further with the cut on the total transverse energy. The $H_{T}$ variable is defined as

$$
H_{T}=\sum_{\text {leptons }} E_{T}+\not_{T}+\sum_{j e t s} E_{T}
$$

where $\sum_{\text {leptons }} E_{T}$ is the scalar sum of transverse energy of all leptons passing the identification and isolation requirement. The missing $E_{T}$ is corrected as described in Section 3.3.3. The $\sum_{j e t s} E_{T}$ is the scalar sum of all jet candidates described in Section 3.3.4. The $t \bar{t}$ events usually have larger $H_{T}$ compared to other Standard Model dilepton backgrounds. The $H_{T}$ distributions of the di-boson production which will be discussed in the next chapter are shown in Figures 4.13, 4.14, and 4.15. These indicate that the cut on the $H_{T}$ can remove a large fraction of the backgrounds while losing only a tiny fraction of $t \bar{t}$ events as shown in Figures 3.10, 3.11, and 3.12 with the requirement of $H_{T}>200 \mathrm{GeV}$.

After all dilepton selections are applied, the events where two leptons have the same sign charges are removed. This reduces the backgrounds from fake leptons by a factor of two because there is no charge correlation in this type of background. In addition, the $W$ 's from $t \bar{t}$ decays have opposite charges and therefore so should the two leptons. 

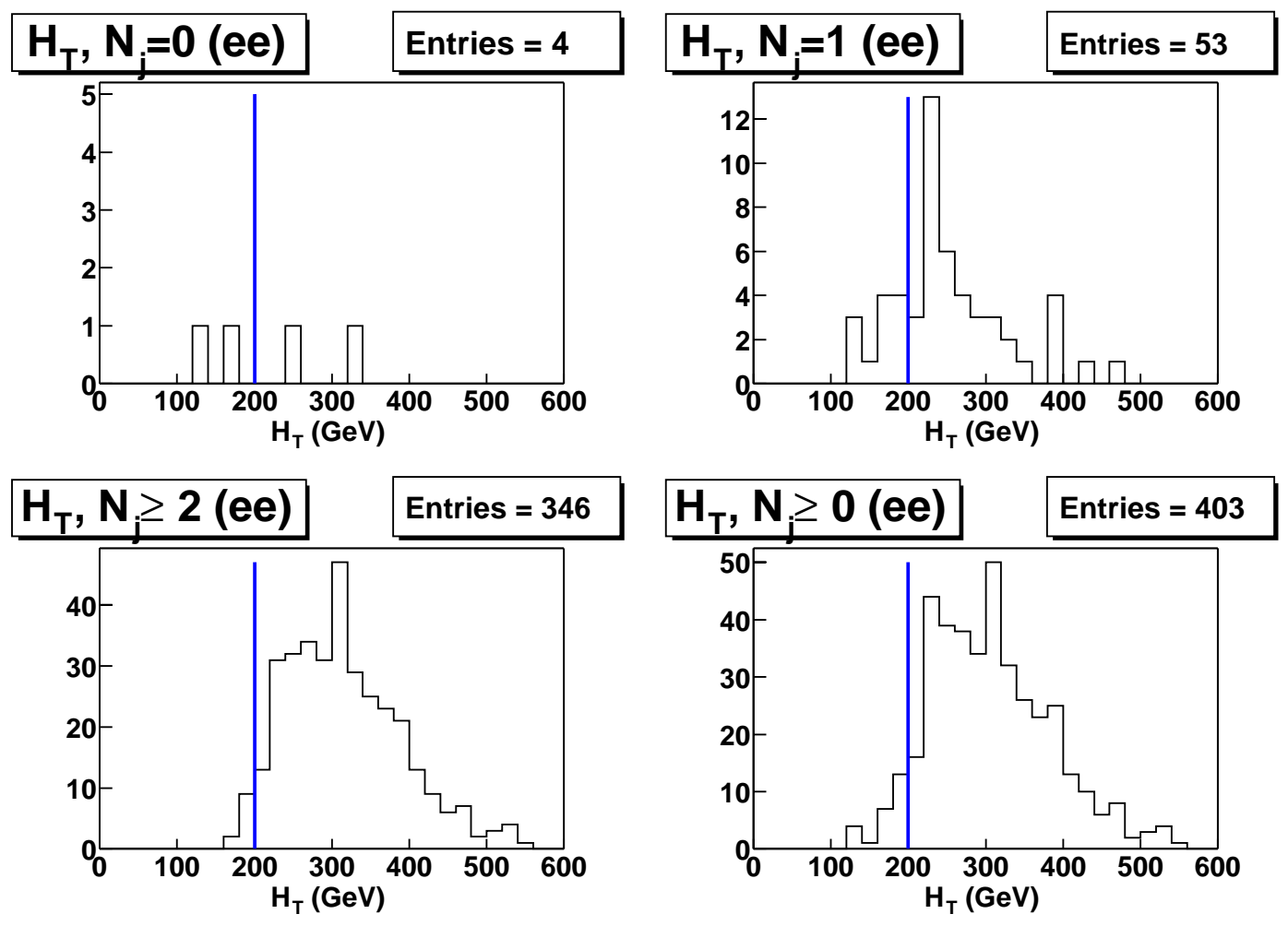

Figure 3.10: The $H_{T}$ of di-electron events in the $t \bar{t}$ PYTHIA Monte Carlo sample with $m_{t}=175 \mathrm{GeV} / \mathrm{c}^{2}$ after $E_{T}$ requirement. The plots are shown with no jet ( $N_{j}=0$, top left), $N_{j}=1$ (top right), $N_{j} \geq 2$ (bottom left), $N_{j} \geq 0$ (bottom right). The line shows the the cut for dilepton events $\left(H_{T}>200 \mathrm{GeV}\right)$. Only the events with $N_{j} \geq 2$ are selected for the final $t \bar{t}$ dilepton events. 

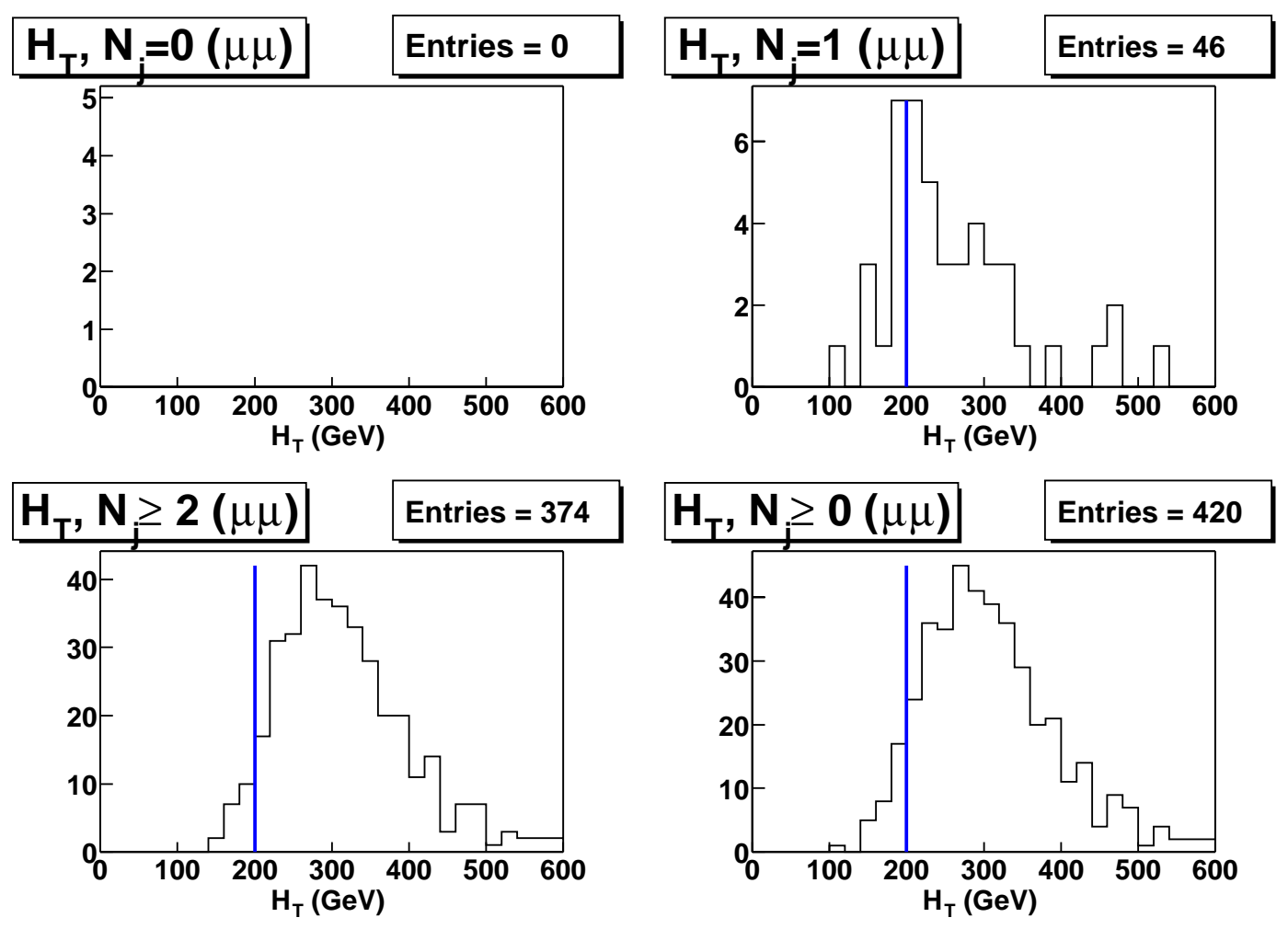

Figure 3.11: The $H_{T}$ of di-muon events in the $t \bar{t}$ PYTHIA Monte Carlo sample with $m_{t}=175 \mathrm{GeV} / \mathrm{c}^{2}$ after $\mathbb{E}_{T}$ requirement. The plots are shown with no jet $\left(N_{j}=0\right.$, top left), $N_{j}=1$ (top right), $N_{j} \geq 2$ (bottom left), $N_{j} \geq 0$ (bottom right). The line shows the the cut for dilepton events $\left(H_{T}>200 \mathrm{GeV}\right)$. Only the events with $N_{j} \geq 2$ are selected for the final $t \bar{t}$ dilepton events. 

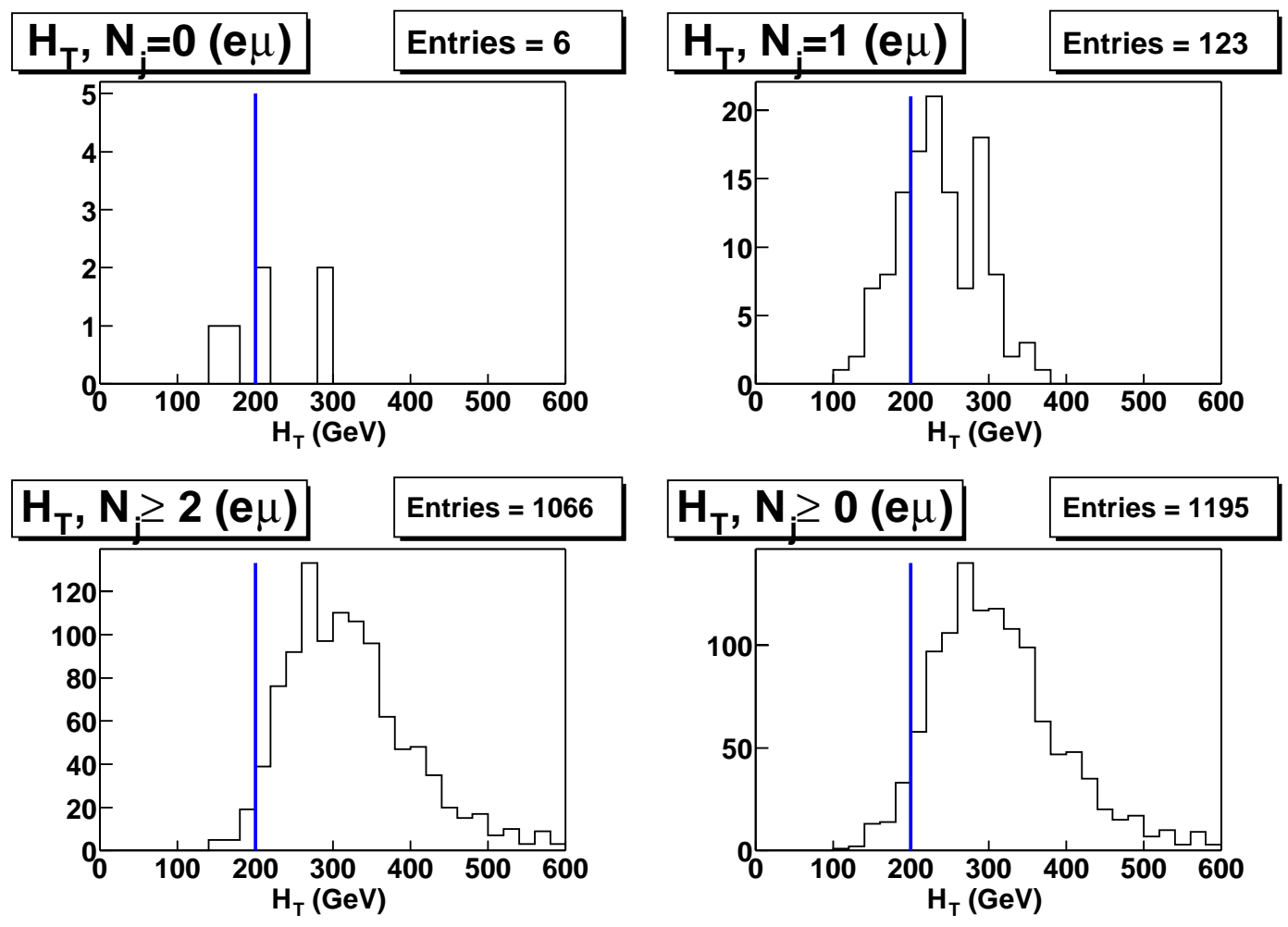

Figure 3.12: The $H_{T}$ of $e \mu$ events in the $t \bar{t}$ PYTHIA Monte Carlo sample with $m_{t}=175 \mathrm{GeV} / \mathrm{c}^{2}$ after $\mathbb{E}_{T}$ requirement. The plots are shown with no jet $\left(N_{j}=0\right.$, top left), $N_{j}=1$ (top right), $N_{j} \geq 2$ (bottom left), $N_{j} \geq 0$ (bottom right). The line shows the the cut for dilepton events $\left(H_{T}>200 \mathrm{GeV}\right)$. Only the events with $N_{j} \geq 2$ are selected for the final $t \bar{t}$ dilepton events. 


\subsubsection{Considerations for cross section measurement}

The dilepton selection requirements discussed in this chapter provide the baseline of the $t \bar{t}$ dilepton channel analysis. However, it is possible to modify the scenario to provide more sensitivity for the cross section measurement. The possible alternatives include allowing a non-isolated lepton in one of the two leptons in the dilepton events and removing the $Z^{0}$ invariant mass cut. The plug electron introduces a substantial uncertainty in the cross section measurement because of uncertainties associated with estimating the background contributions from fake leptons. The removal of dilepton categories involving the plug electrons therefore reduces the systematic uncertainty. In general, loosened cuts and the inclusion of more lepton categories result in an increase of $t \bar{t}$ events surviving event selection. However, there are additional background events as well. The increase of $t \bar{t}$ events from the larger acceptance is beneficial in reducing the statistical error of the cross section measurement, but increases the systematic uncertainty due to the uncertainty in the background estimation.

It is difficult to determine which scenario is better based solely on the signalto-background ratio or the $t \bar{t}$ acceptance. To investigate each scenario, a technique based on pseudoexperiments is exploited. A detailed description of this technique is presented in Appendix B. From the study of Appendix B, a scenario with 12 dilepton categories as shown in Table 3.5 is the most promising one in the high luminosity era. Therefore, only this scenario will be considered in the subsequent parts of this thesis. 


\begin{tabular}{c|c}
\hline & Dilepton Category \\
\hline \hline$e-e$ & TCE - TCE \\
\hline \multirow{5}{*}{$\mu-\mu$} & CMUP - CMUP \\
& CMUP - CMU \\
& CMUP - CMP \\
& CMUP - CMX \\
& CMX - CMU \\
& CMX - CMP \\
& CMX - CMX \\
\hline \multirow{3}{*}{$e-\mu$} & TCE - CMUP \\
& TCE - CMU \\
& TCE - CMP \\
& TCE - CMX \\
\hline
\end{tabular}

Table 3.5: Dilepton categories for $e e, \mu \mu, e \mu$ without PHX, PEM and CMIO.

\subsection{Dilepton Channel Acceptance}

The dilepton channel acceptance of the $t \bar{t}$ pair production is defined as the fraction of $t \bar{t}$ events that pass all the dilepton selection cuts described previously. The dilepton acceptance is dominated by the events in which both $W$ bosons decay leptonically. However, there are small contributions from the lepton + jets channel where one of the $b$ quarks decays semileptonically to produce a high $p_{T}$ lepton. The channel where one $W$ boson decays to $\tau$, then $\tau \rightarrow \ell \nu_{\ell} \nu_{\tau}$, also contributes to the dilepton acceptance. The dilepton acceptance is obtained from the $t \bar{t}$ Monte Carlo sample. The number of events in the $t \bar{t}$ Monte Carlo sample that pass all the dilepton selection cuts is divided by the total number of events in the sample to give the dilepton channel acceptance. 


\subsubsection{Monte Carlo Samples}

The PYTHIA Monte Carlo generator is used to obtain a $t \bar{t}$ sample with $m_{t}=$ $175 \mathrm{GeV} / \mathrm{c}^{2}$. There are no constraints in the decays of the top quark. The sample includes all possible decay channels of $t \bar{t}$ events. A PYTHIA sample of 366,366 events was obtained using version 6.203 of the PYTHIA code [38] with the CTEQ5L structure functions [39]. A sample using the HERWIG Monte Carlo generator is used for the purpose of comparison in the calculation of the acceptance and the study of the systematic uncertainty. For this sample, version 6.4 of the HERWIG code [40] [41] was used to obtain $365,769 t \bar{t}$ events.

\subsubsection{Acceptance and Kinematic Properties of $t \bar{t}$ events}

The leptons decaying from $W$ bosons are high $p_{T}$ and most of them are distributed in the central $\eta$ region. Figure 3.13 shows the $p_{T}$ and $\eta$ distribution of the leptons from $W$ bosons at the generator level (before the detector simulation) of the $t \bar{t}$ Monte Carlo sample. Roughly $85 \%$ of the leptons pass the $p_{T}>20 \mathrm{GeV} / \mathrm{c}$ cut and about $75 \%$ of the leptons are in the central region $(|\eta|<1.1)$. Even though the requirement of both leptons with $p_{T}>20 \mathrm{GeV} / \mathrm{c}$ effectively selects the dilepton events where both $W$ bosons decay leptonically, a small number of events from the lepton + jets channels where one of the leptons comes from the $W$ boson and the other from a semileptonic $b$-quark decay are still present. In addition, there are some dilepton events where one of the $W$ bosons decays to a $\tau$ lepton and subsequently the $\tau$ decays to an electron or muon. Figures 3.1 and 3.2 show the $E_{T}$ distributions of the leptons from the various decay sources in the Monte Carlo $t \bar{t}$ sample. 
After lepton identification requirements, about $7 \%$ of the dilepton events involve a $b$ decay lepton and $12 \%$ of the dilepton events have leptons from $\tau$ 's (Table 3.6). The isolation requirement further reduces the contributions of the $b$ decay leptons. As shown in Figures 3.3 and 3.4, the leptons from $W$ or $\tau$ are well isolated. However, the leptons from $b$ decays are poorly isolated and most of them fail the isolation requirement $I s o 4<0.1$. After the isolation requirement, the contributions from $b$ semileptonic decays are reduced to negligible levels as shown in Table 3.7. The result of applying the dilepton selection cuts on the PYTHIA $t \bar{t}$ sample is listed in Table 3.8 which shows the number of events passing each dilepton selection cut. Table 3.9 shows the cut efficiencies at each step of the dilepton selection.

\subsubsection{Lepton Identification Efficiency}

Ideally, the acceptance of the $t \bar{t}$ events could be calculated by passing the Monte Carlo $t \bar{t}$ sample through the dilepton selection cuts and counting the surviving events. However, there are discrepancies between the efficiencies for passing the lepton identification requirements in the real data versus the Monte Carlo sample. This is because the Monte Carlo detector simulation does not perfectly mimic the leptons in the real detector. The differences in the lepton identification efficiencies in the real data and the Monte Carlo sample are displayed in Table A.17 with the ratios of the efficiencies in the data to the Monte Carlo samples. The lepton identification efficiencies in Table A.17 are calculated with the $Z^{0} \rightarrow \ell \ell$ sample from the data and the Monte Carlo sample respectively. The lepton pairs obtained from the events that have the invariant mass of two leptons between $75 \mathrm{GeV} / \mathrm{c}^{2}$ and 

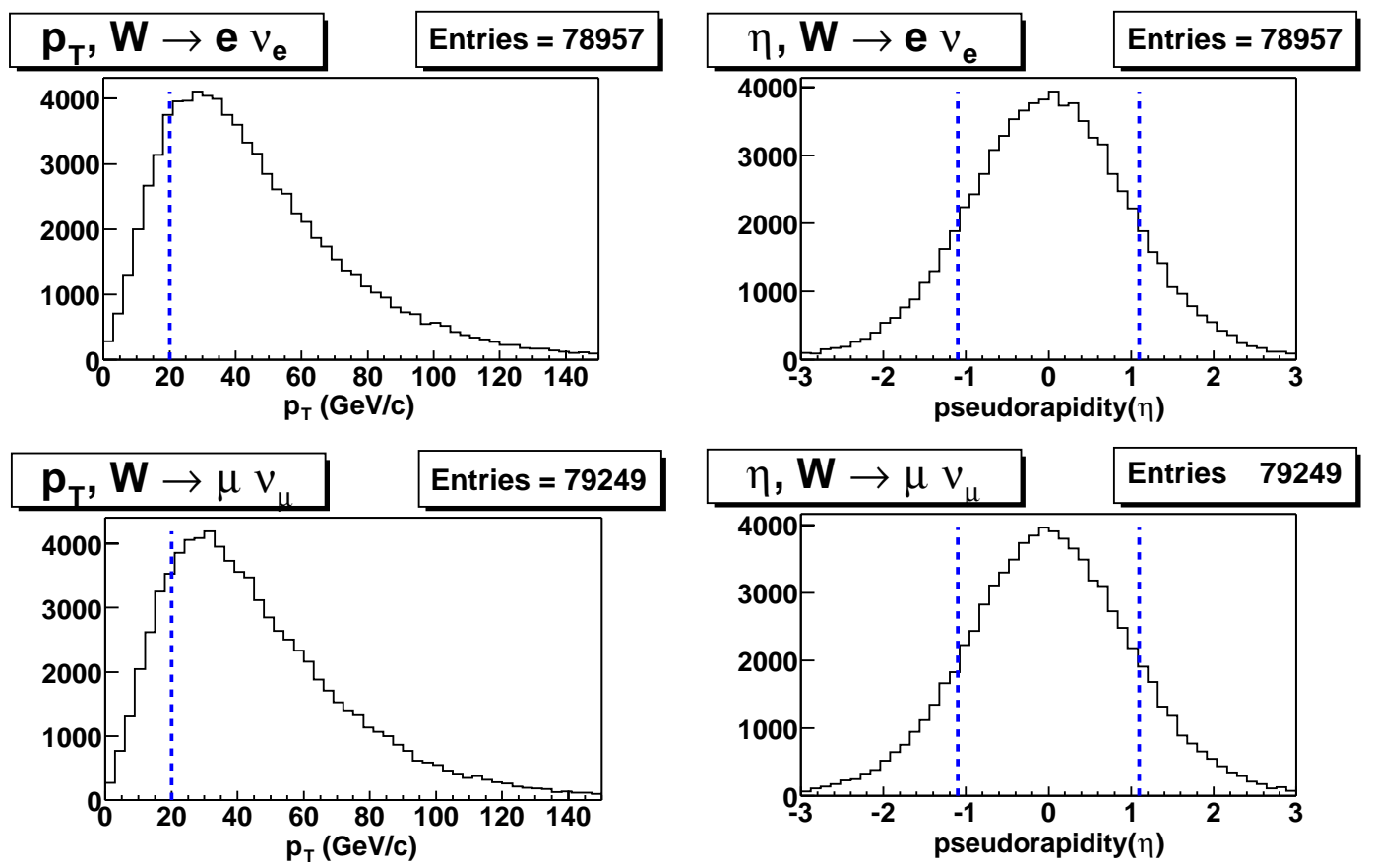

Figure 3.13: The $p_{T}$ and $\eta$ distributions of the leptons from the $W$ decay at the generator level of the $t \bar{t}$ PYTHIA Monte Carlo sample with $m_{t}=175 \mathrm{GeV} / \mathrm{c}^{2}$. Electron $p_{T}$ (top left), $\eta$ (top right), muon $p_{T}$ (bottom left), $\eta$ (bottom right). 


\begin{tabular}{c|ccc}
\hline$t \bar{t}$ dilepton source & \multicolumn{3}{|c}{ Dilepton Category (\%) } \\
$W W \rightarrow$ & $e e$ & $\mu \mu$ & $e \mu$ \\
\hline \hline$e e$ & $76.0 \pm 1.4$ & 0 & $0.6 \pm 0.2$ \\
$\mu \mu$ & 0 & $77.0 \pm 1.4$ & $0.7 \pm 0.2$ \\
$\tau \tau$ & $0.5 \pm 0.2$ & $0.3 \pm 0.2$ & $0.6 \pm 0.2$ \\
$e \mu$ & $2.2 \pm 0.5$ & $1.5 \pm 0.4$ & $77.4 \pm 1.0$ \\
$e \tau$ & $12.3 \pm 1.1$ & $0.2 \pm 0.1$ & $6.9 \pm 0.6$ \\
$\mu \tau$ & 0 & $12.2 \pm 1.1$ & $6.4 \pm 0.6$ \\
\hline$e j j$ & $8.4 \pm 0.9$ & 0 & $3.2 \pm 0.4$ \\
$\mu j j$ & 0 & $8.3 \pm 0.9$ & $3.6 \pm 0.4$ \\
$\tau j j$ & $0.6 \pm 0.3$ & $0.5 \pm 0.2$ & $0.6 \pm 0.2$ \\
\hline$e j j(b \rightarrow \ell)$ & $7.5 \pm 0.9$ & 0 & $2.8 \pm 0.4$ \\
$\mu j j(b \rightarrow \ell)$ & 0 & $7.6 \pm 0.9$ & $3.2 \pm 0.4$ \\
$\tau j j(b \rightarrow \ell)$ & $0.5 \pm 0.2$ & $0.4 \pm 0.2$ & $0.4 \pm 0.1$ \\
\hline$j j j j$ & $0.1 \pm 0.1$ & 0 & $0.1 \pm 0.1$ \\
\hline
\end{tabular}

Table 3.6: The relative acceptances to the $t \bar{t}$ dilepton signal (for the $e e, \mu \mu, e \mu$ separately) from various decay channels $(W W \rightarrow e e, \mu \mu, \ldots)$. The first six rows show the relative acceptances when $W W$ decays to two leptons at the generator level. The next three rows correspond to one $W$ decaying to a lepton, the other decaying hadronically. In the next three rows, $(b \rightarrow \ell)$ means that a $b$-quark decays semileptonically. The last row corresponds to the all hadronic decays of the $W W$. All the numbers are counted after only the lepton identification requirements. Errors are statistical only. 


\begin{tabular}{c|ccc}
\hline$t \bar{t}$ dilepton source & \multicolumn{3}{|c}{ Dilepton Category (\%) } \\
$W W \rightarrow$ & $e e$ & $\mu \mu$ & $e \mu$ \\
\hline \hline$e e$ & $84.4 \pm 1.4$ & 0 & $0.1 \pm 0.1$ \\
$\mu \mu$ & 0 & $87.4 \pm 1.2$ & $0.3 \pm 0.1$ \\
$\tau \tau$ & $0.4 \pm 0.3$ & $0.4 \pm 0.2$ & $0.6 \pm 0.2$ \\
$e \mu$ & $0.7 \pm 0.3$ & 0 & $84.2 \pm 0.9$ \\
$e \tau$ & $12.5 \pm 1.3$ & 0 & $7.4 \pm 0.7$ \\
$\mu \tau$ & 0 & $11.9 \pm 1.2$ & $6.8 \pm 0.7$ \\
\hline$e j j$ & $1.5 \pm 0.5$ & 0 & $0.1 \pm 0.1$ \\
$\mu j j$ & 0 & $0.1 \pm 0.1$ & $0.5 \pm 0.2$ \\
$\tau j j$ & $0.4 \pm 0.3$ & $0.1 \pm 0.1$ & $0.1 \pm 0.1$ \\
\hline$e j j(b \rightarrow \ell)$ & $1.5 \pm 0.5$ & 0 & $0.1 \pm 0.1$ \\
$\mu j j(b \rightarrow \ell)$ & 0 & $0.1 \pm 0.1$ & $0.3 \pm 0.1$ \\
$\tau j j(b \rightarrow \ell)$ & $0.3 \pm 0.2$ & $0.1 \pm 0.1$ & $0.1 \pm 0.1$ \\
\hline$j j j j$ & 0 & 0 & 0 \\
\hline
\end{tabular}

Table 3.7: The relative acceptances to the $t \bar{t}$ dilepton signal (for the $e e, \mu \mu, e \mu$ separately) from various decay channels $(W W \rightarrow e e, \mu \mu, \ldots)$. The first six rows show the relative acceptances when $W W$ decays to two leptons at the generator level. The next three rows correspond to one $W$ decaying to a lepton, the other decaying hadronically. In the next three rows, $(b \rightarrow \ell)$ means that a $b$-quark decays semileptonically. The last row corresponds to the all hadronic decays of the $W W$. All the numbers are counted after only the lepton identification requirements and the isolation requirement. Errors are statistical only. 


\begin{tabular}{c|ccccccccc}
\hline Category & lep ID & Isol & $Z^{0}$ mass & $E_{T}$ & \multicolumn{3}{c}{$N_{j}$} & \multirow{2}{*}{$H_{T}$} & OS \\
\cline { 6 - 8 } & & & & & 0 & 1 & $\geq 2$ & & \\
\hline \hline TCE TCE & 883 & 679 & 493 & 403 & 4 & 53 & 346 & 335 & 330 \\
\hline \hline CMUP CMUP & 319 & 260 & 186 & 148 & 0 & 25 & 123 & 112 & 112 \\
CMUP CMU & 116 & 77 & 51 & 40 & 0 & 4 & 36 & 35 & 35 \\
CMUP CMP & 177 & 130 & 95 & 70 & 0 & 7 & 63 & 60 & 60 \\
CMUP CMX & 214 & 171 & 129 & 100 & 0 & 8 & 92 & 89 & 89 \\
CMX CMU & 38 & 24 & 19 & 16 & 0 & 2 & 14 & 14 & 14 \\
CMX CMP & 67 & 50 & 37 & 28 & 0 & 0 & 28 & 27 & 27 \\
CMX CMX & 35 & 27 & 22 & 18 & 0 & 0 & 18 & 18 & 18 \\
\hline$\mu \mu$ & 965 & 739 & 539 & 420 & 0 & 46 & 374 & 355 & 355 \\
\hline \hline TCE CMUP & 1079 & 853 & & 668 & 4 & 72 & 592 & 577 & 573 \\
TCE CMU & 188 & 147 & & 118 & 1 & 9 & 108 & 105 & 105 \\
TCE CMP & 314 & 229 & & 188 & 0 & 16 & 172 & 168 & 167 \\
TCE CMX & 348 & 266 & & 221 & 1 & 26 & 194 & 187 & 186 \\
\hline$e \mu$ & 1925 & 1495 & & 1195 & 6 & 123 & 1066 & 1037 & 1031 \\
\hline \hline total & 3758 & 2913 & 2527 & 2018 & 10 & 222 & 1786 & 1727 & 1716 \\
\hline \hline
\end{tabular}

Table 3.8: Results from the dilepton selection on the $t \bar{t}$ PYTHIA sample $\left(m_{t}=\right.$ $175 \mathrm{GeV} / \mathrm{c}^{2}, 366,366$ events). 


\begin{tabular}{c|cccccc}
\hline \multirow{2}{*}{ Category } & \multicolumn{5}{|c}{ Cut efficiencies (\%) } \\
\cline { 2 - 7 } & Isol & $Z^{0}$ mass & $\not_{T}$ & $N_{j} \geq 2$ & $H_{T}$ & OS \\
\hline \hline TCE TCE & $76.9 \pm 1.4$ & $72.6 \pm 1.7$ & $81.7 \pm 1.7$ & $85.9 \pm 1.7$ & $96.8 \pm 0.9$ & $98.5 \pm 0.7$ \\
\hline \hline CMUP CMUP & $81.5 \pm 2.2$ & $71.5 \pm 2.8$ & $79.6 \pm 3.0$ & $83.1 \pm 3.1$ & $91.1 \pm 2.6$ & 100.0 \\
CMUP CMU & $66.4 \pm 4.4$ & $66.2 \pm 5.4$ & $78.4 \pm 5.8$ & $90.0 \pm 4.7$ & $97.2 \pm 2.7$ & 100.0 \\
CMUP CMP & $73.4 \pm 3.3$ & $73.1 \pm 3.9$ & $73.7 \pm 4.5$ & $90.0 \pm 3.6$ & $95.2 \pm 2.7$ & 100.0 \\
CMUP CMX & $79.9 \pm 2.7$ & $75.4 \pm 3.3$ & $77.5 \pm 3.7$ & $92.0 \pm 2.7$ & $96.7 \pm 1.9$ & 100.0 \\
CMX CMU & $63.2 \pm 7.8$ & $79.2 \pm 8.3$ & $84.2 \pm 8.4$ & $87.5 \pm 8.3$ & 100.0 & 100.0 \\
CMX CMP & $74.6 \pm 5.3$ & $74.0 \pm 6.2$ & $75.7 \pm 7.1$ & 100.0 & $96.4 \pm 3.5$ & 100.0 \\
CMX CMX & $77.1 \pm 7.1$ & $81.5 \pm 7.5$ & $81.8 \pm 8.2$ & 100.0 & 100.0 & 100.0 \\
\hline$\mu \mu$ & $76.6 \pm 1.4$ & $72.9 \pm 1.6$ & $77.9 \pm 1.8$ & $89.0 \pm 1.5$ & $94.9 \pm 1.1$ & 100.0 \\
\hline \hline TCE CMUP & $79.1 \pm 1.2$ & & $78.3 \pm 1.4$ & $88.6 \pm 1.2$ & $97.5 \pm 0.6$ & $99.3 \pm 0.3$ \\
TCE CMU & $78.2 \pm 3.0$ & & $80.3 \pm 3.3$ & $91.5 \pm 2.6$ & $97.2 \pm 1.6$ & 100.0 \\
TCE CMP & $72.9 \pm 2.5$ & & $82.1 \pm 2.5$ & $91.5 \pm 2.0$ & $97.7 \pm 1.1$ & $99.4 \pm 0.6$ \\
TCE CMX & $76.4 \pm 2.3$ & & $83.1 \pm 2.3$ & $87.8 \pm 2.2$ & $96.4 \pm 1.3$ & $99.5 \pm 0.5$ \\
\hline$e \mu$ & $77.7 \pm 0.9$ & & $79.9 \pm 1.0$ & $89.2 \pm 0.9$ & $97.3 \pm 0.5$ & $99.4 \pm 0.2$ \\
\hline \hline total & $77.5 \pm 0.7$ & $86.7 \pm 0.6$ & $79.9 \pm 0.8$ & $88.5 \pm 0.7$ & $96.7 \pm 0.4$ & $99.4 \pm 0.2$ \\
\hline
\end{tabular}

Table 3.9: The cut efficiencies after the lepton identification on the $t \bar{t}$ PYTHIA sample $\left(m_{t}=175 \mathrm{GeV} / \mathrm{c}^{2}, 366,366\right.$ events). The efficiencies are calculated from the events that passed all preceding dilepton selections. All errors are statistical only. 
$105 \mathrm{GeV} / \mathrm{c}^{2}$ provide a true lepton sample with high purity. The method of calculating the lepton identification efficiencies using the leptons obtained from this sample is explained in Appendix A.

The differences in the lepton identification efficiencies are applied to the calculation of the $t \bar{t}$ acceptance to obtain a better estimation. As shown in Table A.17, each lepton type has a different scale factor. Therefore each dilepton event is assigned a different weight, $w=s($ lepton 1$) \times s($ lepton 2$)$, where $s$ is the scale factor for that lepton type. The weighted number of events are counted to determine the final acceptance value. The numbers of events weighted in this way are displayed in Table 3.10. The final acceptance number calculated with the weighted number of events is shown in the last row of Table 3.11.

\subsubsection{Systematic Uncertainty}

There are several sources of systematic uncertainty in the dilepton $t \bar{t}$ acceptance that are introduced by the imperfect modeling of $t \bar{t}$ production and the detector simulation. As discussed in Section 3.4.3, the lepton identification efficiency is one of the largest sources of the systematic uncertainty. The acceptance that is multiplied with the weight described in Section 3.4.3 is used for the final acceptance. The systematic uncertainty caused by the lepton identification efficiency is simply taken as half of the difference between the acceptance without the weight correction and the weighted acceptance. The uncertainty from this source is $4.0 \%$.

The jet energy estimation as discussed in Section 3.3.4 also provides a source of uncertainty for the dilepton acceptance. To estimate the uncertainty from the jet 


\begin{tabular}{c|cc}
\hline Category & After all dilepton cuts & Weighted for lepton ID \\
\hline \hline TCE TCE & 330 & 311.2 \\
\hline CMUP CMUP & 112 & 95.2 \\
CMUP CMU & 35 & 31.0 \\
CMUP CMP & 60 & 54.2 \\
CMUP CMX & 89 & 83.5 \\
CMX CMU & 14 & 13.7 \\
CMX CMP & 27 & 26.9 \\
CMX CMX & 18 & 18.6 \\
$\mu \mu$ & 355 & 323.0 \\
\hline \hline TCE CMUP & 573 & 513.1 \\
TCE CMU & 105 & 97.8 \\
TCE CMP & 167 & 158.8 \\
TCE CMX & 186 & 183.7 \\
\hline$e \mu$ & 1031 & 953.4 \\
\hline \hline total & 1716 & 1587.6 \\
\hline
\end{tabular}

Table 3.10: Result from the dilepton selection on the $t \bar{t}$ PYTHIA sample $\left(m_{t}=\right.$ $175 \mathrm{GeV} / \mathrm{c}^{2}, 366,366$ events). The numbers in the last column are obtained by multiplying the weighting factor to the numbers of events passing all dilepton selections in the second column. 


\begin{tabular}{c|c}
\hline Cuts & Cumulative cut efficiencies (\%) \\
\hline \hline$\epsilon_{I D}$ & $1.03 \pm 0.02$ \\
Fiducial $/ p_{T} \&$ Lepton ID \\
(incl. cosmic \& conversion) & \\
\hline Isolation & $77.5 \pm 0.7$ \\
$Z^{0}$ mass $\left(m_{\ell \ell}\right)$ & $86.7 \pm 0.6$ \\
$\not_{T}$ & $79.9 \pm 0.8$ \\
$N_{j} \geq 2$ & $88.5 \pm 0.7$ \\
$H_{T}$ & $96.7 \pm 0.4$ \\
Opposite Sign Charge & $99.4 \pm 0.2$ \\
\hline total dilepton $\left(\epsilon_{\text {dil }}\right)$ & $0.47 \pm 0.01$ \\
\hline & \\
\hline & \\
\hline & \\
\hline
\end{tabular}

Table 3.11: The acceptance of fiducial, $p_{T}$ and lepton identification $\left(\epsilon_{I D}\right)$ and the cut efficiencies after the lepton identification requirements. The final dilepton acceptance $\left(\epsilon_{d i l}\right)$ is the probability that a $t \bar{t}$ event passes all the dilepton selection requirements. The number in the last row is the dilepton acceptance with the identification efficiency correction. All errors are statistical only. 
energy scale, the jet energies in the $t \bar{t}$ events in the Monte Carlo sample were increased and decreased by $\pm 1 \sigma$ from their central value provided by the jet algorithm. The PYTHIA $t \bar{t}$ sample gives

$$
\begin{gathered}
\epsilon_{d i l}=0.487 \pm 0.01 \%\left(E_{T}^{\prime}(j e t)=E_{T}(j e t)+\Delta E(+1 \sigma)\right) \\
\epsilon_{d i l}=0.444 \pm 0.01 \%\left(E_{T}^{\prime}(j e t)=E_{T}(j e t)+\Delta E(-1 \sigma)\right),
\end{gathered}
$$

where the acceptances are not weighted as described in the previous section. The uncertainty from the jet energy scale is $4.6 \%$ by taking the half of the difference.

Structure functions also introduce a systematic uncertainty. The default $t \bar{t}$ Monte Carlo sample in this analysis uses CTEQ5L structure function [42]. With $\Lambda_{Q C D}=$ $300 \mathrm{MeV}$, two Monte Carlo samples using parton distribution functions CTEQ5L and MRST were used to estimate the acceptance difference caused by using different structure functions. The difference between the two samples gives a $6.8 \%$ uncertainty. The acceptances with different values of $\Lambda_{Q C D}$ but the same structure function were compared as well. The Monte Carlo samples using the same structure function, MRST, were prepared with $\Lambda_{Q C D}=300 \mathrm{MeV}$ and $\Lambda_{Q C D}=229 \mathrm{MeV}$ respectively. The difference between two samples results in a systematic error of $0.8 \%$. From these two comparisons, the uncertainty due to the structure functions is assigned as $6.8 \%$.

Different Monte Carlo generators give slightly different values of acceptance. To compare the acceptances from different generators, the HERWIG $t \bar{t}$ sample and 
PYTHIA $t \bar{t}$ sample were prepared. Because the HERWIG generator does not model the QED final state radiation (FSR) [43], the PYTHIA sample without QED FSR was used. The difference in this comparison gives an uncertainty of $3.4 \%$.

The initial state gluon radiation and final state gluon radiation simulated by the Monte Carlo generator affect the acceptance as well. The PYTHIA $t \bar{t}$ sample with the initial state radiation turned off is compared with the default PYTHIA sample with the initial state and final state radiation turned on. The comparison of the different initial state radiation settings gives a 3.1\% uncetainty. Because it is not possible to turn off the final state gluon radiation in the current generators the comparison on different final state radiation settings was not performed in this thesis [44].

The trigger effect on the acceptance was not investigated thoroughly in this thesis because it is expected to be very small. The effect of the central electron triggers can be neglected because their efficiencies are almost 100\%. In the previous studies of the muon trigger effect on the $t \bar{t}$ acceptance in Run I [45], the $t \bar{t}$ acceptance decreased by about $2 \%$ due to the muon trigger. In the current estimate of the $t \bar{t}$ acceptance, an uncertainty of $2 \%$ is assigned for the trigger effect.

The total systematic error in the $t \bar{t}$ acceptance, $\epsilon_{d i l}$, is estimated to be $10.4 \%$. The sources of systematic errors discussed in this section are listed in Table 3.12. The $t \bar{t}$ dilepton acceptance estimated in this chapter with the statistical and systematic errors is

$$
\epsilon_{\text {dil }}=0.43 \pm 0.01(\text { stat }) \pm 0.04(\text { syst }) \% .
$$




\begin{tabular}{c|c}
\hline Sources & Error (\%) \\
\hline \hline & \\
Lepton ID efficiencies & $4.0 \%$ \\
Jet energy scale & $4.6 \%$ \\
Structure functions & $6.8 \%$ \\
Monte Carlo generator & $3.4 \%$ \\
ISR & $3.1 \%$ \\
Trigger efficiency & $2.0 \%$ \\
& \\
\hline Total & $10.4 \%$ \\
\hline
\end{tabular}

Table 3.12: Contributions from various sources of systematic uncertainty to the dilepton acceptance. 


\section{CHAPTER 4}

\section{BACKGROUNDS IN DILEPTON EVENTS}

The selection cuts introduced in the previous chapter are designed to remove most of the dilepton events which are not due to $t \bar{t}$ production while retaining the $t \bar{t}$ events as much as possible. The background events with at least two jets are usually related to higher order QCD processes beyond the tree level. Therefore, the two jet requirement alone reduces the contributions from the non- $t \bar{t}$ backgrounds significantly. Furthermore, the $\mathbb{E}_{T}$ and the invariant mass cut significantly reduce the backgrounds from the Drell-Yan and $Z^{0} \rightarrow \tau \tau$ processes. Additionally, the $H_{T}$ cut is useful to discriminate the $t \bar{t}$ events from the backgrounds since $t \bar{t}$ events have decay products with larger transverse energy. In this chapter, the estimation of the number of backgrounds events remaining after selection is discussed.

\subsection{Drell-Yan process}

The Drell-Yan process is one of the main sources of backgrounds in the $t \bar{t}$ dilepton channel [46]. In this process, electron or muon pair production is mediated by a virtual photon or a $Z$ boson from the $q \bar{q}$ annihilation (Figure 4.1). The dilepton events observed in the data are dominated by the events originating from a real $Z^{0}$ 


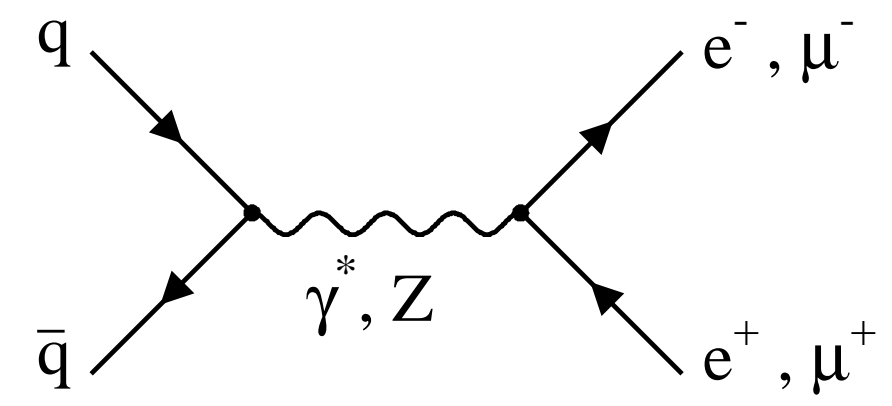

Figure 4.1: Drell-Yan process.

decay which have a dilepton invariant mass around $90 \mathrm{GeV} / \mathrm{c}^{2}$ as shown in Figure 3.5. The simple and efficient way to remove the Drell-Yan contribution is to impose an invariant mass cut of $m_{\ell \ell}<76 \mathrm{GeV} / \mathrm{c}^{2}$ or $m_{\ell \ell}>106 \mathrm{GeV} / \mathrm{c}^{2}$.

The Drell-Yan process does not have real missing energy because it does not have a neutrino in the final state. Therefore, the $\mathbb{E}_{T}$ observed in the Drell-Yan process usually has a small magnitude, which is not sufficient to pass the $\mathbb{E}_{T}$ requirement. The distributions of $E_{T}\left(x\right.$ axis) are shown in Figures 4.2 and 4.3 for the $Z^{0} \rightarrow e^{+} e^{-}$, $\mu^{+} \mu^{-}$data $\left(76 \mathrm{GeV} / \mathrm{c}^{2}<m_{\ell \ell}<106 \mathrm{GeV} / \mathrm{c}^{2}\right)$ of $109 \mathrm{pb}^{-1}$.

The critical task for the estimation of this background is to understand the fraction of Drell-Yan events that have a $\mathscr{E}_{T} \geq 25 \mathrm{GeV}$. This $\mathbb{E}_{T}$ mainly originates 
from the mismeasurement of jet energies. Therefore it is important to understand the tail of the $\mathbb{E}_{T}$ resolution. Since the tails of resolution functions can be difficult to model in Monte Carlo, a data sample is used to estimate the Drell-Yan background instead.

The background contribution from the Drell-Yan is estimated by the following method [47][48]. This method assumes that the Drell-Yan event possesses similar kinematic properties regardless of its dilepton invariant mass. We start by selecting Drell-Yan events $\left(N_{Z^{0}}\right)$ in the data that fall in the dilepton invariant mass range $76 \mathrm{GeV} / \mathrm{c}^{2}<m_{\ell \ell}<106 \mathrm{GeV} / \mathrm{c}^{2}$. Although these events are typically removed by the $m_{\ell \ell}$ cut, we apply all other dilepton selection and count the number of events that pass $\left(N_{Z^{0}}^{\text {pass }}\right)$. The ratio of $N_{Z^{0}}^{\text {pass }}$ to $N_{Z^{0}}$ represents the fraction of Drell-Yan events that will satisfy the $E_{T}$ and other cuts. This fraction is applied to the events that fall outside the $m_{\ell \ell}$ window. This is expressed by the following equation.

$$
N_{b g}^{D Y}=N_{Z^{0}}^{\text {pass }} \frac{N_{l o}+N_{h i}}{N_{Z^{0}}}
$$

The $N_{l o}$ and $N_{h i}$ are the number of events without the dilepton selection cuts $\left(\mathbb{E}_{T}, 2\right.$ jets, $H_{T}$, opposite sign $)$ in the low $\left(m_{\ell \ell}<76 \mathrm{GeV} / \mathrm{c}^{2}\right)$ and high $\left(m_{\ell \ell}>106 \mathrm{GeV} / \mathrm{c}^{2}\right)$ invariant mass region. In this calculation, it is assumed that the cut efficiencies of the dilepton selections are the same regardless of the dilepton invariant mass.

This approximation is quite good; however, studies have shown that the cut efficiencies for two jets and $H_{T}$ have some dependence on the dilepton invariant mass. Therefore, additional corrections are applied to Equation 4.1. The dependence of 

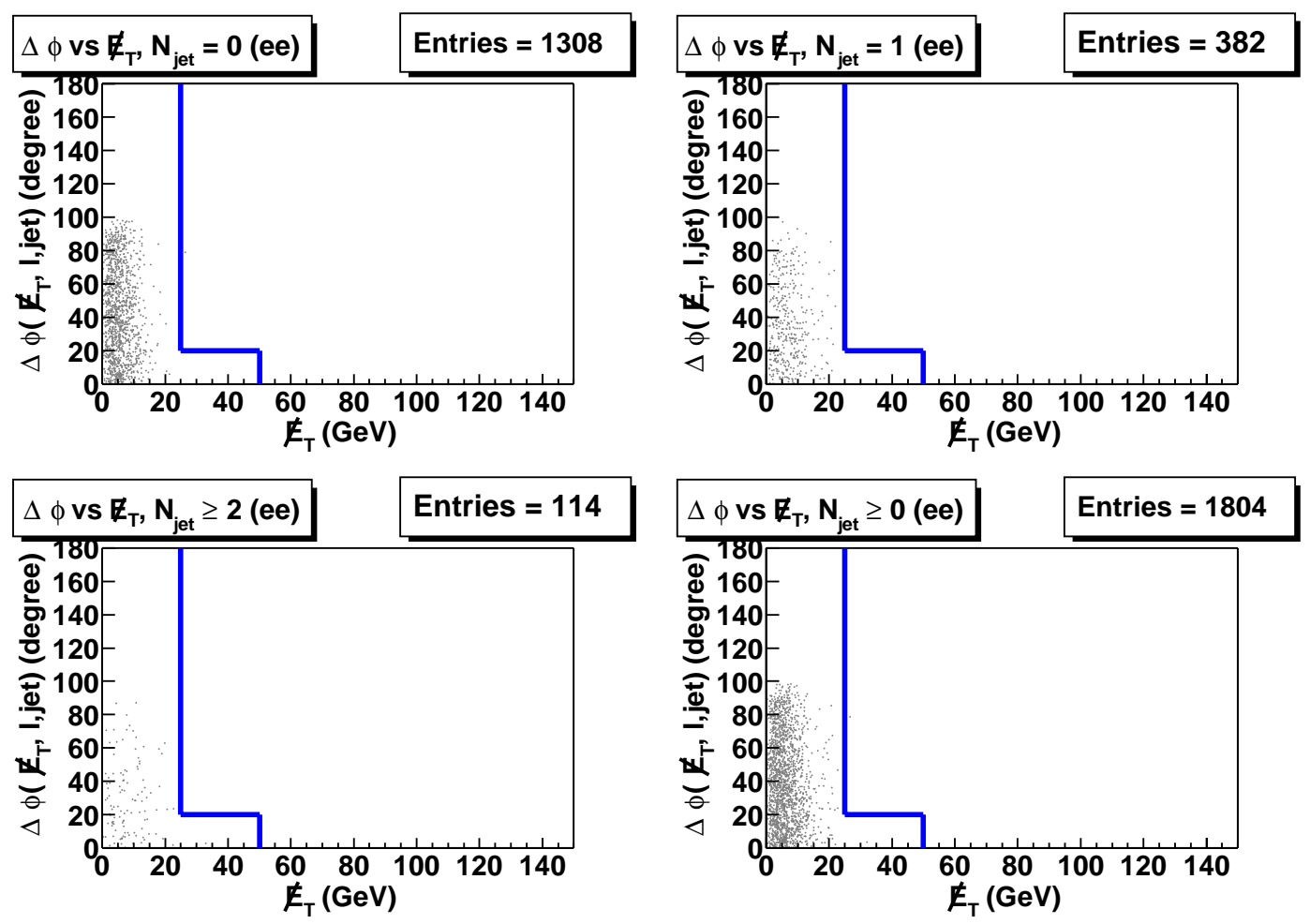

Figure 4.2: Azimuthal angle between the $\mathbb{E}_{T}$ and the closest lepton or jet, versus the $\not_{T}$ for $Z^{0} \rightarrow e^{+} e^{-}$data $\left(109 p b^{-1}\right)$. 

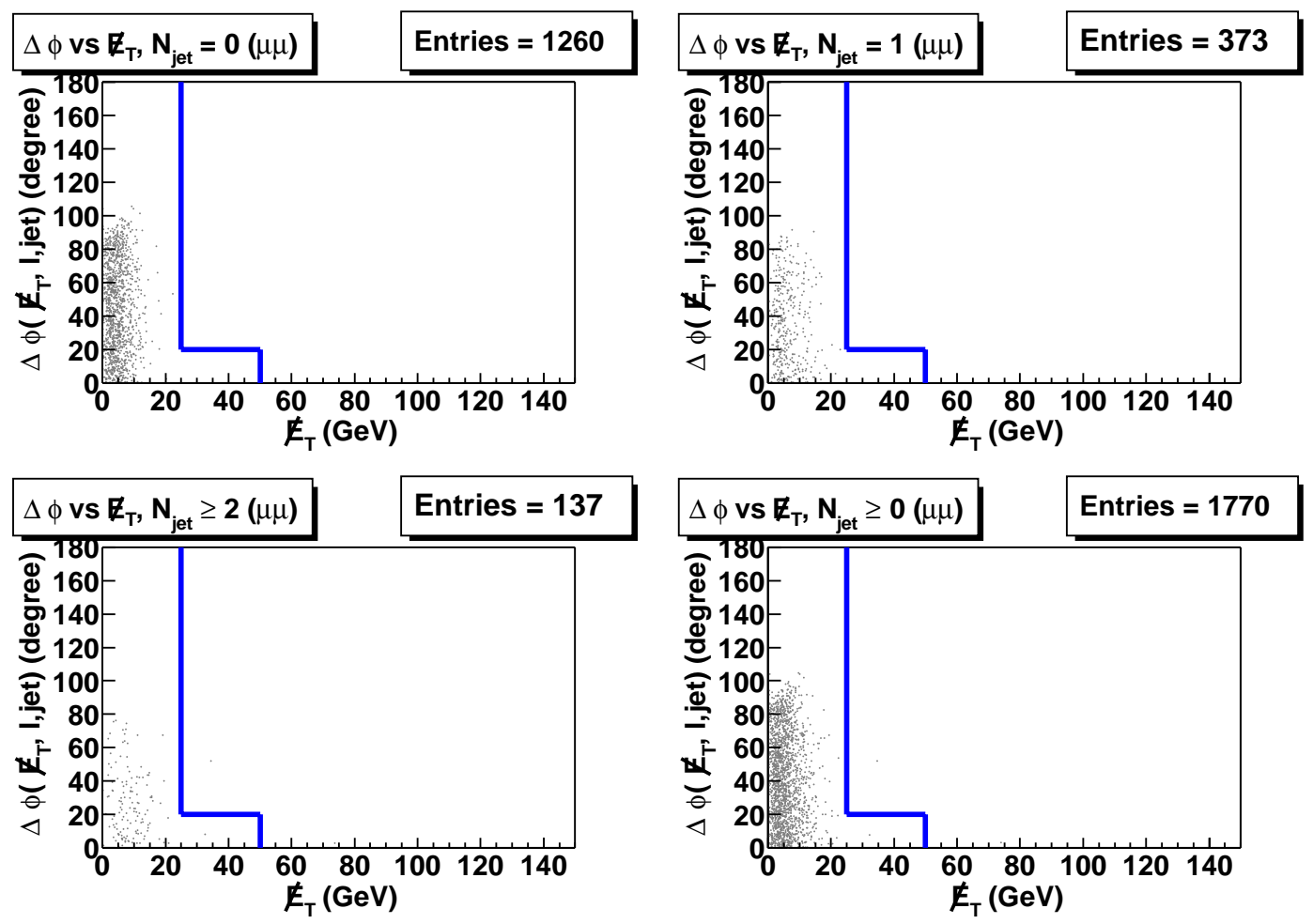

Figure 4.3: Azimuthal angle between the $\mathbb{E}_{T}$ and the closest lepton or jet, versus the $\not_{T}$ for $Z^{0} \rightarrow \mu^{+} \mu^{-}$data $\left(109 p b^{-1}\right)$. 
the two-jet cut on the invariant mass had been studied using $Z+2$ jets matrix element Monte Carlo. The empirical equation of the efficiency of Drell-Yan 2-jet cut [49] is given by

$$
\epsilon_{2 j}=\left(2.5 \times 10^{-4}\right) m_{\ell \ell}(G e V)+9.6 \times 10^{-3} .
$$

The $H_{T}$ cut efficiency of Drell-Yan events is obtained from the PYTHIA Drell-Yan Monte Carlo sample. The numbers of events before the $H_{T}$ cut and after the $H_{T}$ cut are counted in this sample as shown in Table 4.1 to give the efficiencies. With the cut efficiencies on the two-jet and $H_{T}$, the number of Drell-Yan backgrounds is given by

$$
N_{b g}^{D Y}=N_{Z^{0}}^{p a s s} \frac{\epsilon_{2 j, l o} \epsilon_{H_{T}, l o} N_{l o}+\epsilon_{2 j, h i} \epsilon_{H_{T}, h i} N_{h i}}{\epsilon_{2 j, Z^{0}} \epsilon_{H_{T}, Z^{0}} N_{Z^{0}}}
$$

where $\epsilon_{H_{T}, Z^{0}}$ is the $H_{T}$ cut efficiency on the events with the dilepton invariant mass inside the $Z^{0}$ mass peak. Other efficiencies are defined in a similar way.

The number of events $N_{Z^{0}}^{\text {pass }}$ includes a contribution from the $t \bar{t}$ events inside the $Z^{0}$ mass region. This is not a negligible portion in the total number of events after applying the $E_{T}$ cut. The $t \bar{t}$ contribution from the $Z^{0}$ mass region is estimated from the PYTHIA Monte Carlo sample. The estimated $t \bar{t}$ contribution is then subtracted from the $N_{Z^{0}}^{\text {pass }}$ in the final determination of the Drell-Yan background in Equation 4.3. In Table 4.2, the number of events used for the Drell-Yan background estimation, and the estimated results are shown. 


\begin{tabular}{c|cc|cc|cc}
\hline & \multicolumn{2}{|l|}{$m_{\ell \ell}<76 \mathrm{GeV} / \mathrm{c}^{2}$} & \multicolumn{2}{|c|}{$\begin{array}{c}76 \mathrm{GeV} / \mathrm{c}^{2}<m_{\ell \ell} \\
<106 \mathrm{GeV} / \mathrm{c}^{2}\end{array}$} & $m_{\ell \ell}>106 \mathrm{GeV} / \mathrm{c}^{2}$ \\
\cline { 2 - 7 } & $e e$ & $\mu \mu$ & $e e$ & $\mu \mu$ & $e e$ & $\mu \mu$ \\
\hline \hline before $H_{T}$ & 0 & 0 & 7 & 11 & 0 & 3 \\
after $H_{T}$ & 0 & 0 & 5 & 9 & 0 & 3 \\
\hline$\epsilon_{H_{T}}$ & - & - & 0.71 & 0.82 & - & 1.0 \\
\hline
\end{tabular}

Table 4.1: The numbers of events before the $H_{T}$ cut and after the $H_{T}$ cut using the PYTHIA Drell-Yan Monte Carlo sample $\left(2.3 \mathrm{fb}^{-1}\right.$ for the electron channel, $2.6 \mathrm{fb}^{-1}$ for the muon channel). $\epsilon_{H_{T}}$ is the $H_{T}$ cut efficiency with $H_{T}>200 \mathrm{GeV}$.

\begin{tabular}{c|ccc|ccc|c|c|c}
\hline & $N_{l o}$ & $N_{Z^{0}}$ & $N_{h i}$ & $\left\langle m_{\ell \ell}\right\rangle_{l o}$ & $\left\langle m_{\ell \ell}\right\rangle_{Z^{0}}$ & $\left\langle m_{\ell \ell}\right\rangle_{h i}$ & $N_{Z^{0}}^{p a s s}$ & $N_{Z^{0}}^{t \bar{t}}$ & $N_{b g}^{D Y}$ \\
\hline \hline$e e$ & 172 & 1804 & 66 & 59.7 & 90.8 & 141.3 & 0 & 0.25 & - \\
$\mu \mu$ & 174 & 1770 & 67 & 51.1 & 90.7 & 131.2 & 2 & 0.25 & $0.11 \pm 0.09$ \\
\hline
\end{tabular}

Table 4.2: The numbers of events used for the Drell-Yan background calculation and the result in $109 \mathrm{pb}^{-1}$ of data. $\left\langle m_{\ell \ell}\right\rangle$ is the mean dilepton invariant mass. The $N_{Z^{0}}^{\text {pass }}$ is the number of events inside the $Z^{0}$ mass peak passing all the dilepton cuts. The $N_{Z^{0}}^{t \bar{t}}$ is the number of the $t \bar{t}$ events estimated with the PYTHIA $t \bar{t}$ Monte Carlo sample inside the $Z^{0}$ mass peak and passing all the dilepton cuts. The $N_{b g}^{D Y}$ is the estimated background from the Drell-Yan process in $109 p b^{-1}$ of data. 


\section{$4.2 \quad Z^{0} \rightarrow \tau^{+} \tau^{-}$}

The dilepton events involving the $Z^{0}$ boson include the decay of $Z^{0} \rightarrow \tau^{+} \tau^{-}$ because the $\tau$ can decay to the electron or muon via $\tau \rightarrow \ell \overline{\nu_{\ell}} \nu_{\tau}$. When jets from the higher order processes are included, $Z^{0} \rightarrow \tau^{+} \tau^{-}$is a source of the background for the $t \bar{t}$ dilepton channel. In this case, there may be a real missing energy from the four neutrinos. However, normally the $\mathbb{E}_{T}$ from the neutrinos is small because the events tend to have a back-to-back topology for the two $\tau$ 's. Therefore, the contribution from the neutrinos will tend to cancel. This can be seen in the distributions of the $Z^{0} \rightarrow \tau^{+} \tau^{-}$where the events are populated in the low $\mathbb{E}_{T}$ region as shown in Figures 4.4, 4.5 and 4.6.

Another noticeable feature of the $Z^{0} \rightarrow \tau^{+} \tau^{-}$events is that the $\mathbb{E}_{T}$ tends to align parallel with the leptons in the event. The population of the events with a small $\Delta \phi$ between $\mathbb{E}_{T}$ and the closest lepton extends even into the higher $\mathbb{E}_{T}$ region. This $\Delta \phi$ vs $E_{T}$ cut, introduced in the dilepton selection requirements, is motivated by these observations.

To estimate this type of background, a PYTHIA $Z^{0} \rightarrow \tau^{+} \tau^{-}$sample with 416,499 events is used. The sample contains only the events which have two leptons with $E_{T}>20 \mathrm{GeV}$ and $|\eta|<2.5$ to increase the statistical power after application of the dilepton cuts. The integrated luminosity of the sample is equivalent to $140 \mathrm{fb}^{-1}$. 

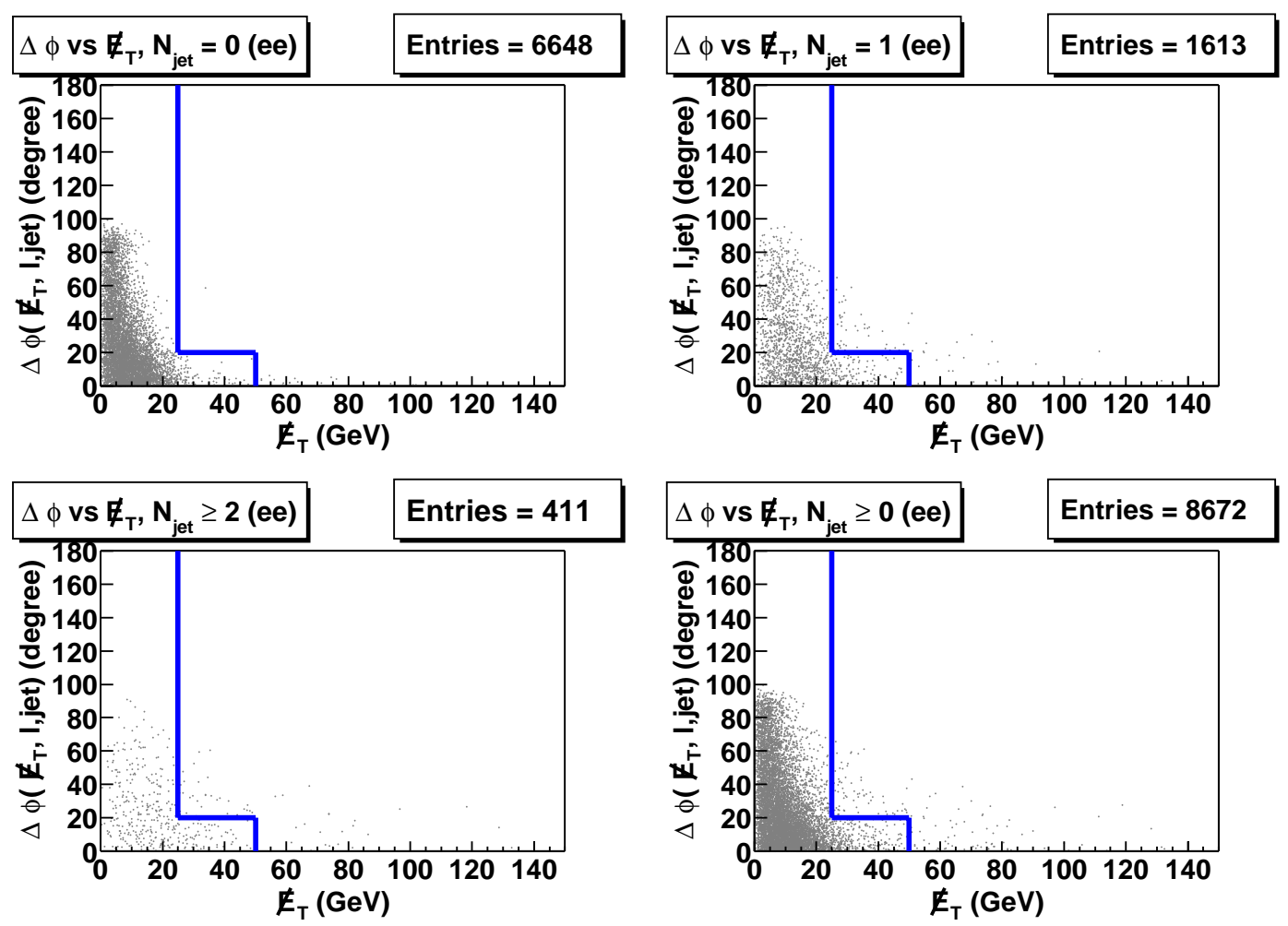

Figure 4.4: The magnitude of the missing $E_{T}\left(E_{T}, x\right.$ axis) and the $\Delta \phi$ between $E_{T}$ and the closest jet or lepton ( $y$ axis). Di-electron events in the $Z^{0} \rightarrow \tau^{+} \tau^{-}$PYTHIA Monte Carlo sample are shown with no jet $\left(N_{j}=0\right.$, top left), $N_{j}=1$ (top right), $N_{j} \geq 2$ (bottom left), $N_{j} \geq 0$ (bottom right). The lines show the cut imposed for the $\not_{T}$ requirement. 

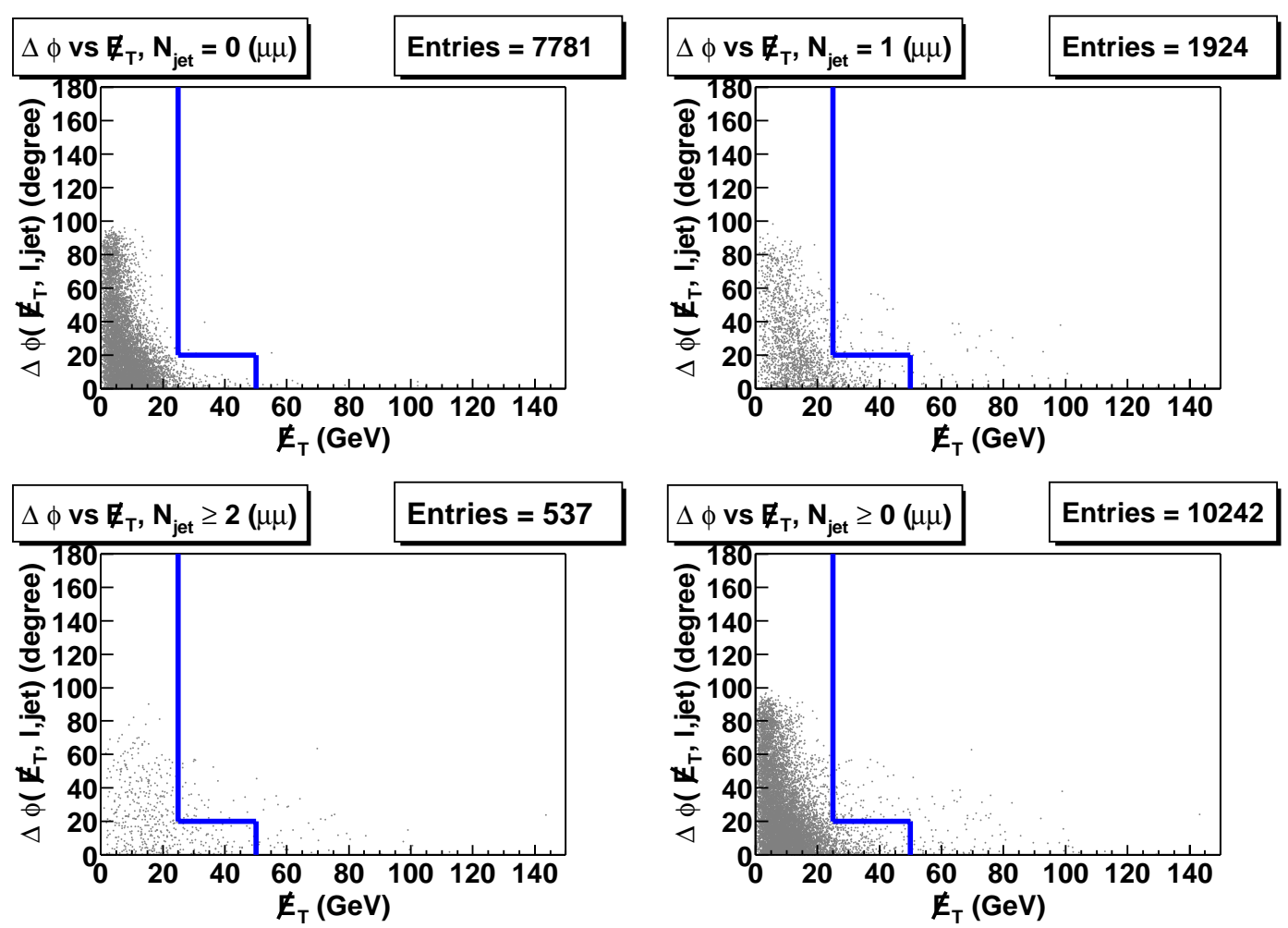

Figure 4.5: The magnitude of the missing $E_{T}\left(E_{T}, x\right.$ axis) and the $\Delta \phi$ between $\not_{T}$ and the closest jet or lepton ( $y$ axis). Di-muon events in the $Z^{0} \rightarrow \tau^{+} \tau^{-}$PYTHIA Monte Carlo sample are shown with no jet $\left(N_{j}=0\right.$, top left), $N_{j}=1$ (top right), $N_{j} \geq 2$ (bottom left), $N_{j} \geq 0$ (bottom right). The lines show the cut imposed for the $\not_{T}$ requirement. 

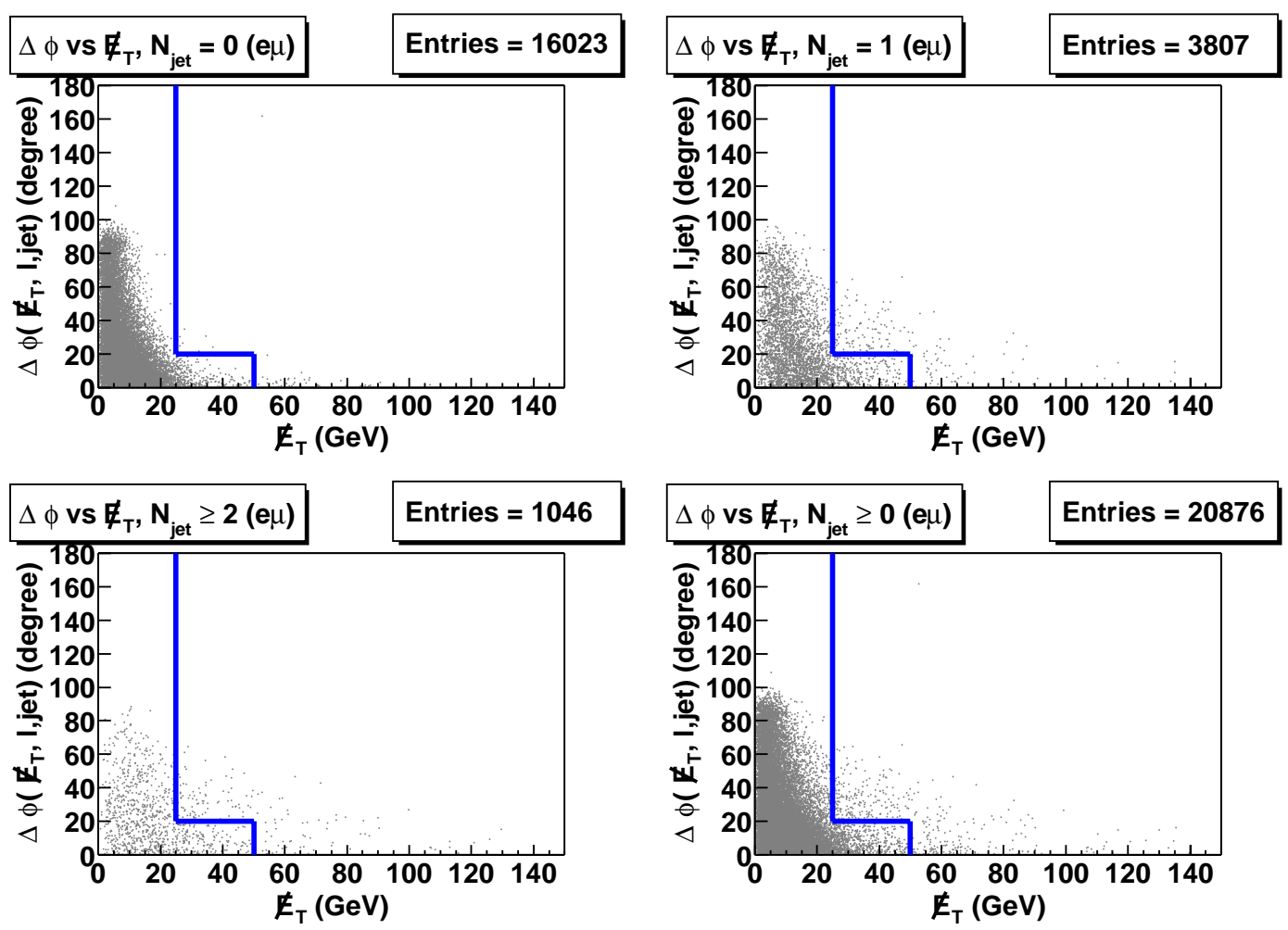

Figure 4.6: The magnitude of the missing $E_{T}\left(E_{T}, x\right.$ axis) and the $\Delta \phi$ between $Z_{T}$ and the closest jet or lepton ( $y$ axis). $e \mu$ events in the $Z^{0} \rightarrow \tau^{+} \tau^{-}$PYTHIA Monte Carlo sample are shown with no jet $\left(N_{j}=0\right.$, top left), $N_{j}=1$ (top right), $N_{j} \geq 2$ (bottom left), $N_{j} \geq 0$ (bottom right). The lines show the cut imposed for the $\mathbb{E}_{T}$ requirement. 
The dilepton selection is applied to the sample and the results are given in Table 4.3. The number of events in Table 4.3 are used to estimate $Z^{0} \rightarrow \tau^{+} \tau^{-}$backgrounds in $109 p b^{-1}$

$$
\begin{aligned}
& N_{b g}^{Z \rightarrow \tau \tau}(e e)=0.03 \pm 0.01 \\
& N_{b g}^{Z \rightarrow \tau \tau}(\mu \mu)=0.04 \pm 0.01 \\
& N_{b g}^{Z \rightarrow \tau \tau}(e \mu)=0.06 \pm 0.03 \\
& N_{b g}^{Z \rightarrow \tau \tau}(\ell \ell)=0.13 \pm 0.05
\end{aligned}
$$

The errors are estimated from the variations of the jet energies in the events. The method is the same as used in the estimation of the systematic error in the $t \bar{t}$ acceptance discussed in Section 3.4.4. The systematic errors are combined with the statistical errors from the Monte Carlo sample.

The invariant mass cut does not significantly reduce the $Z^{0} \rightarrow \tau^{+} \tau^{-}$events. The energy of the $\tau$ is divided into the energy of the electron (or muon) and the energy of the neutrinos. The peak in the distribution of the invariant mass of the lepton pair from $Z^{0} \rightarrow \tau^{+} \tau^{-}$is lower than the peak of the $Z^{0}$ invariant mass, as shown in Figure 4.7. 


\begin{tabular}{|c|c|c|c|c|c|c|c|c|c|}
\hline \multirow[t]{2}{*}{ Category } & \multirow[t]{2}{*}{ lep ID } & \multirow[t]{2}{*}{ Isol } & \multirow[t]{2}{*}{$Z^{0}$ mass } & \multirow[t]{2}{*}{$\mathbb{E}_{T}$} & \multicolumn{3}{|c|}{$N_{j}$} & \multirow[t]{2}{*}{$H_{T}$} & \multirow[t]{2}{*}{$\mathrm{OS}$} \\
\hline & & & & & 0 & 1 & $\geq 2$ & & \\
\hline TCE TCE & 9969 & 9258 & 8672 & 157 & 21 & 78 & 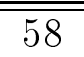 & 34 & 34 \\
\hline ee & 9969 & 9258 & 8672 & 157 & 21 & 78 & 58 & 34 & 34 \\
\hline CMUP CMUP & 3514 & 3280 & 3095 & 42 & 5 & 21 & 16 & 9 & 9 \\
\hline CMUP CMU & 1342 & 1258 & 1188 & 27 & 2 & 12 & 13 & 10 & 10 \\
\hline CMUP CMP & 2066 & 1946 & 1852 & 34 & 3 & 16 & 15 & 10 & 10 \\
\hline CMUP CMX & 2673 & 2486 & 2337 & 48 & 4 & 22 & 22 & 12 & 12 \\
\hline CMX CMU & 606 & 563 & 530 & 7 & 0 & 3 & 4 & 2 & 2 \\
\hline CMX CMP & 720 & 673 & 640 & 12 & 0 & 5 & 7 & 5 & 5 \\
\hline CMX CMX & 678 & 633 & 600 & 10 & 2 & 5 & 3 & 2 & 2 \\
\hline$\mu \mu$ & 11599 & 10839 & 10242 & 180 & 16 & 84 & 80 & 50 & 50 \\
\hline TCE CMUP & 11891 & 11101 & & 205 & 24 & 102 & 79 & 43 & $\overline{43}$ \\
\hline TCE CMU & 2461 & 2291 & & 49 & 13 & 21 & 15 & 6 & 6 \\
\hline TCE CMP & 3314 & 3077 & & 56 & 12 & 25 & 19 & 13 & 13 \\
\hline TCE CMX & 4722 & 4408 & & 97 & 12 & 42 & 43 & 20 & 20 \\
\hline$e \mu$ & 22386 & 20876 & & 407 & 61 & 190 & 156 & 82 & 82 \\
\hline total & 43948 & 40972 & 39789 & 744 & 98 & 352 & 294 & 166 & 166 \\
\hline
\end{tabular}

Table 4.3: Result from the dilepton selection on the PYTHIA $Z^{0} \rightarrow \tau^{+} \tau^{-}$sample (416,499 events). 

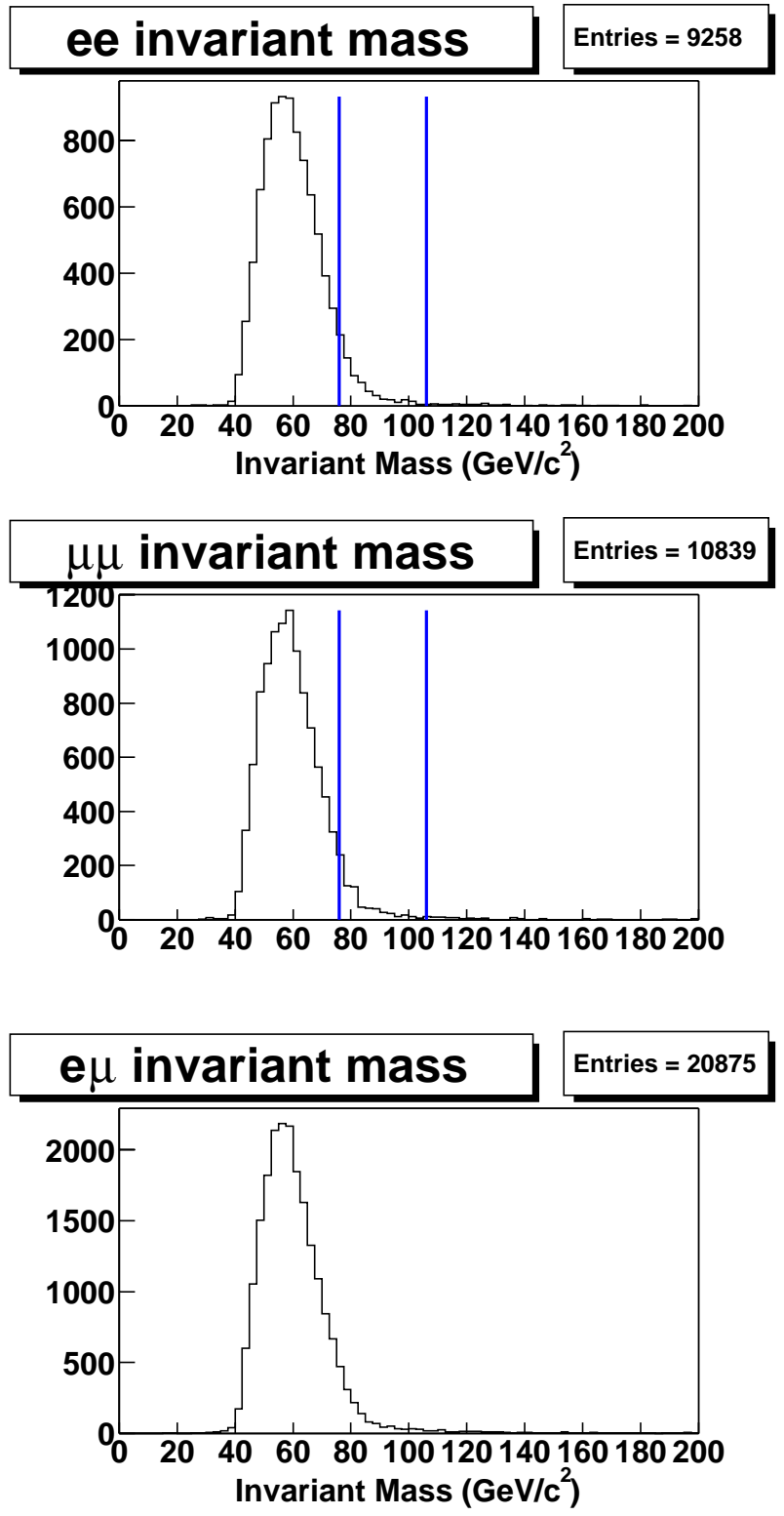

Figure 4.7: Invariant mass of two leptons $\left(m_{\ell \ell}\right)$ in the dilepton events of the PYTHIA $Z^{0} \rightarrow \tau^{+} \tau^{-}$Monte Carlo sample after the isolation requirement is imposed. Dielectron (ee, top), di-muon ( $\mu \mu$, middle), electron-muon (e $\mu$, bottom) events are shown separately. In the $e \mu$ category, the invariant mass cut is not imposed. The red lines in the ee, $e \mu$ show the invariant mass cut of $76 \mathrm{GeV} / \mathrm{c}^{2}<m_{\ell \ell}<106 \mathrm{GeV} / \mathrm{c}^{2}$. 


\subsection{Vector boson pair production}

Pair production of $W$ bosons is possible via $q \bar{q}$ annihilation involving bosonfermion couplings or the tri-boson coupling. The current theoretical calculation of $W W$ production at the next-to-leading order (NLO) gives the following cross sections in the $p \bar{p}$ collisions at $\sqrt{s}=1.96 \mathrm{TeV}$ [51][52];

$$
\sigma(p \bar{p} \rightarrow W W)=13.25 \pm 0.25 p b
$$

Similarly to the previous Standard Model background processes, the higher order processes of $W W$ production involving at least 2 jets are possible candidates for the background of the $t \bar{t}$ dilepton channel. When both of the $W$ bosons decay leptonically, significant $\not_{T}$ results from the two neutrinos and the events will possibly satisfy the dilepton selection requirements. This contribution is estimated using a PYTHIA Monte Carlo sample of $W W$ production. The sample consists of a total 827,323 events where both $W$ bosons are forced to decay leptonically. Using the theoretical cross section, the integrated luminosity of the sample is equivalent to $608.5 \mathrm{fb}^{-1}$.

The dilepton selection is applied to the $W W$ Monte Carlo sample. Because there is real $\mathbb{E}_{T}$ from the two neutrinos, a large fraction of the events passes the $\mathbb{E}_{T}$ cut as shown in Figures 4.9, 4.10 and 4.11. In the distributions for $N_{j}=0$ in Figures 4.9, 4.10 and 4.11, a large fraction of the events in high $\mathbb{E}_{T}$ is found in the large $\Delta \phi$ region. This implies that the two neutrinos tend to be more aligned and more back-to-back with the two leptons for the events in this region. In Figure 4.12, the distributions of the invariant mass of two leptons in the $W W$ events are shown 

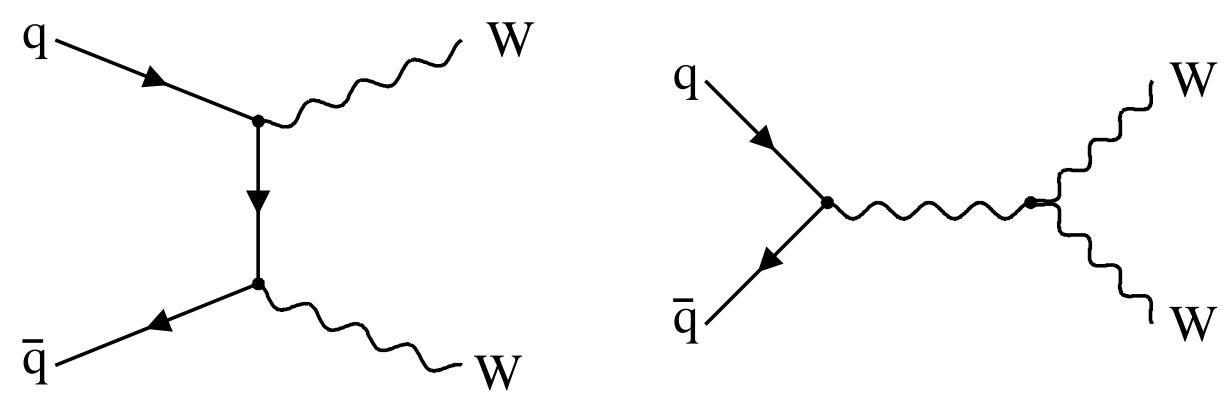

Figure 4.8: Diagrams for the tree level $W W$ production.

with the corresponding dilepton selection cut. The $H_{T}$ cut is effective at separating this background from the $t \bar{t}$ events. As shown in Figures 4.13, 4.14 and 4.15, about $50 \%$ of $W W$ events in the 2 jet bin are removed by requiring $H_{T}>200 \mathrm{GeV}$. In contrast, most of the $t \bar{t}$ events survive, as shown in Figures 3.10, 3.11 and 3.12. The results of applying the dilepton selections to the PYTHIA Monte Carlo sample are listed in Table 4.4. The backgrounds from the $W W$ in $109 p b^{-1}$ are estimated from these results with the errors obtained in a similar way as for the $Z^{0} \rightarrow \tau^{+} \tau^{-}$ background.

$$
\begin{aligned}
& N_{b g}^{W W}(e e)=0.05 \pm 0.01 \\
& N_{b g}^{W W}(\mu \mu)=0.05 \pm 0.02 \\
& N_{b g}^{W W}(e \mu)=0.12 \pm 0.03 \\
& N_{b g}^{W W}(\ell \ell)=0.21 \pm 0.06
\end{aligned}
$$


The other di-boson process $W Z^{0}$ is also a background in the dilepton channel although its cross section is relatively small compared to that of $W W$. The theoretical cross section of $W Z^{0}$ production is $\sigma\left(p \bar{p} \rightarrow W Z^{0}\right)=3.96 \pm 0.06 p b[51]$. The background estimation has been made with a PYTHIA Monte Carlo sample. The Monte Carlo sample contains events passing a loose dilepton selection that demands two leptons $(e, \mu$ or $\tau)$ with $p_{T}>10 \mathrm{GeV}$ and $|\eta|<2.0$. With 73,693 events of the sample, which corresponds to $320 \mathrm{fb}^{-1}$, the background from $W Z^{0}$ has been estimated in a similar way as for $W W$. The estimated background from $W Z^{0}$ is $N_{b g}^{W Z^{0}}=0.04 \pm 0.01$

\subsection{Fake leptons}

The backgrounds in the dilepton channel contain a contribution from events in which a hadron fakes a lepton. This type of background primarily comes from the $W+\geq 3$ jet events where one of the jets is improperly identified as a lepton. Electrons can be faked by highly electromagnetic jets which contain photons or $\pi^{0}$ 's and produce charged tracks pointing to the electromagnetic clusters. Usually, hadronic jets have a poorer isolation than the high $p_{T}$ leptons produced by $W$ decays. The isolation requirement on both leptons rejects most of the backgrounds originated from fake leptons, which are likely non-isolated.

The number of background events caused by fake leptons is extracted from the $W+\geq 3$ jet sample where one of the jets is identified as a lepton. But these events are included in our signal sample and we can not distinguish them from the $t \bar{t}$ events. As an alternative, we look for the $W+\geq 3$ jet events where one of the jets is reconstructed as a lepton but fails the lepton identification requirements. This 


\begin{tabular}{c|ccccccccc}
\hline Category & lep ID & Isol & $Z^{0}$ & $E_{T}$ & \multicolumn{3}{c}{$N_{j}$} & $H_{T}$ & OS \\
\cline { 6 - 8 } & & & mass & & 0 & 1 & $\geq 2$ & & \\
\hline \hline TCE TCE & 11973 & 11156 & 8499 & 6110 & 4372 & 1260 & 478 & 254 & 253 \\
\hline$e e$ & 11973 & 11156 & 8499 & 6110 & 4372 & 1260 & 478 & 254 & 253 \\
\hline \hline CMUP CMUP & 4103 & 3819 & 2942 & 2094 & 1489 & 453 & 152 & 88 & 88 \\
CMUP CMU & 1693 & 1583 & 1206 & 848 & 624 & 172 & 52 & 28 & 28 \\
CMUP CMP & 2321 & 2184 & 1675 & 1192 & 832 & 269 & 91 & 46 & 46 \\
CMUP CMX & 3231 & 3051 & 2311 & 1664 & 1200 & 335 & 129 & 63 & 63 \\
CMX CMU & 693 & 654 & 506 & 357 & 262 & 81 & 14 & 8 & 8 \\
CMX CMP & 914 & 850 & 621 & 465 & 343 & 84 & 38 & 18 & 18 \\
CMX CMX & 658 & 619 & 463 & 288 & 211 & 57 & 20 & 9 & 9 \\
\hline$\mu \mu$ & 13613 & 12760 & 9724 & 6908 & 4961 & 1451 & 496 & 260 & 260 \\
\hline \hline TCE CMUP & 13874 & 12959 & & 8919 & 6433 & 1833 & 653 & 366 & 366 \\
TCE CMU & 2821 & 2642 & & 1844 & 1344 & 392 & 108 & 58 & 58 \\
TCE CMP & 3776 & 3521 & & 2455 & 1769 & 494 & 192 & 110 & 110 \\
TCE CMX & 5579 & 5212 & & 3663 & 2669 & 737 & 257 & 125 & 125 \\
\hline$e \mu$ & 26050 & 24334 & & 16881 & 12215 & 3456 & 1210 & 659 & 659 \\
\hline \hline total & 51635 & 48250 & 42557 & 29899 & 21548 & 6167 & 2184 & 1173 & 1172 \\
\hline
\end{tabular}

Table 4.4: Result from the dilepton selection on the $W W$ PYTHIA sample $(827,323$ events). 

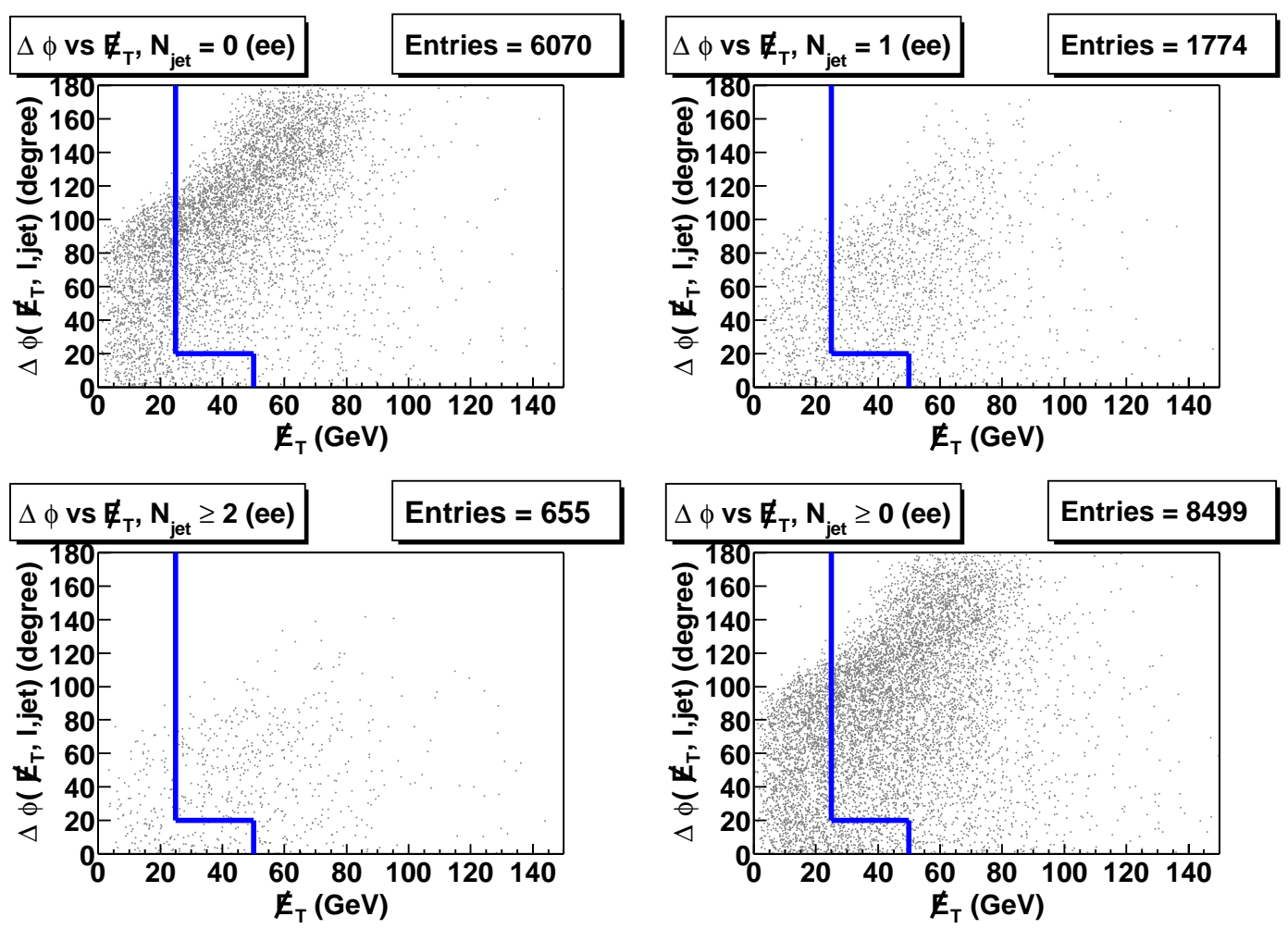

Figure 4.9: The magnitude of the missing $E_{T}\left(E_{T}, x\right.$ axis $)$ and the $\Delta \phi$ between $E_{T}$ and the closest jet or lepton ( $y$ axis). Di-electron events in the $W W$ PYTHIA Monte Carlo sample are shown with no jet $\left(N_{j}=0\right.$, top left), $N_{j}=1$ (top right), $N_{j} \geq 2$ (bottom left), $N_{j} \geq 0$ (bottom right). The lines show the cut imposed for the $\not_{T}$ requirement. This sample is equivalent to $608.5 \mathrm{fb}^{-1}$. 

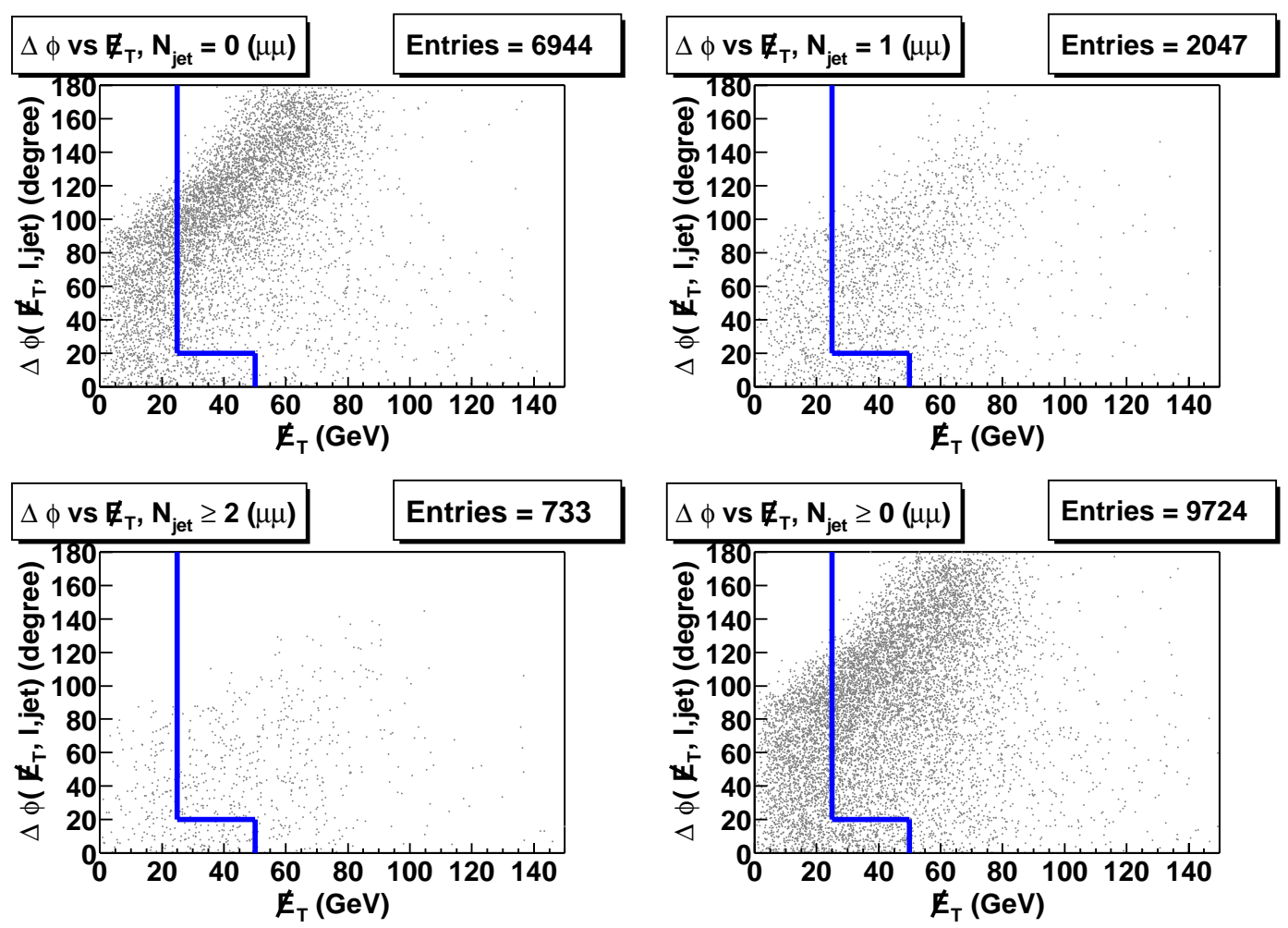

Figure 4.10: The magnitude of the missing $E_{T}\left(E_{T}, x\right.$ axis) and the $\Delta \phi$ between $\not_{T}$ and the closest jet or lepton ( $y$ axis). Di-muon events in the $W W$ PYTHIA Monte Carlo sample are shown with no jet $\left(N_{j}=0\right.$, top left), $N_{j}=1$ (top right), $N_{j} \geq 2$ (bottom left), $N_{j} \geq 0$ (bottom right). The lines show the cut imposed for the $\mathbb{E}_{T}$ requirement. This sample is equivalent to $608.5 \mathrm{fb}^{-1}$. 

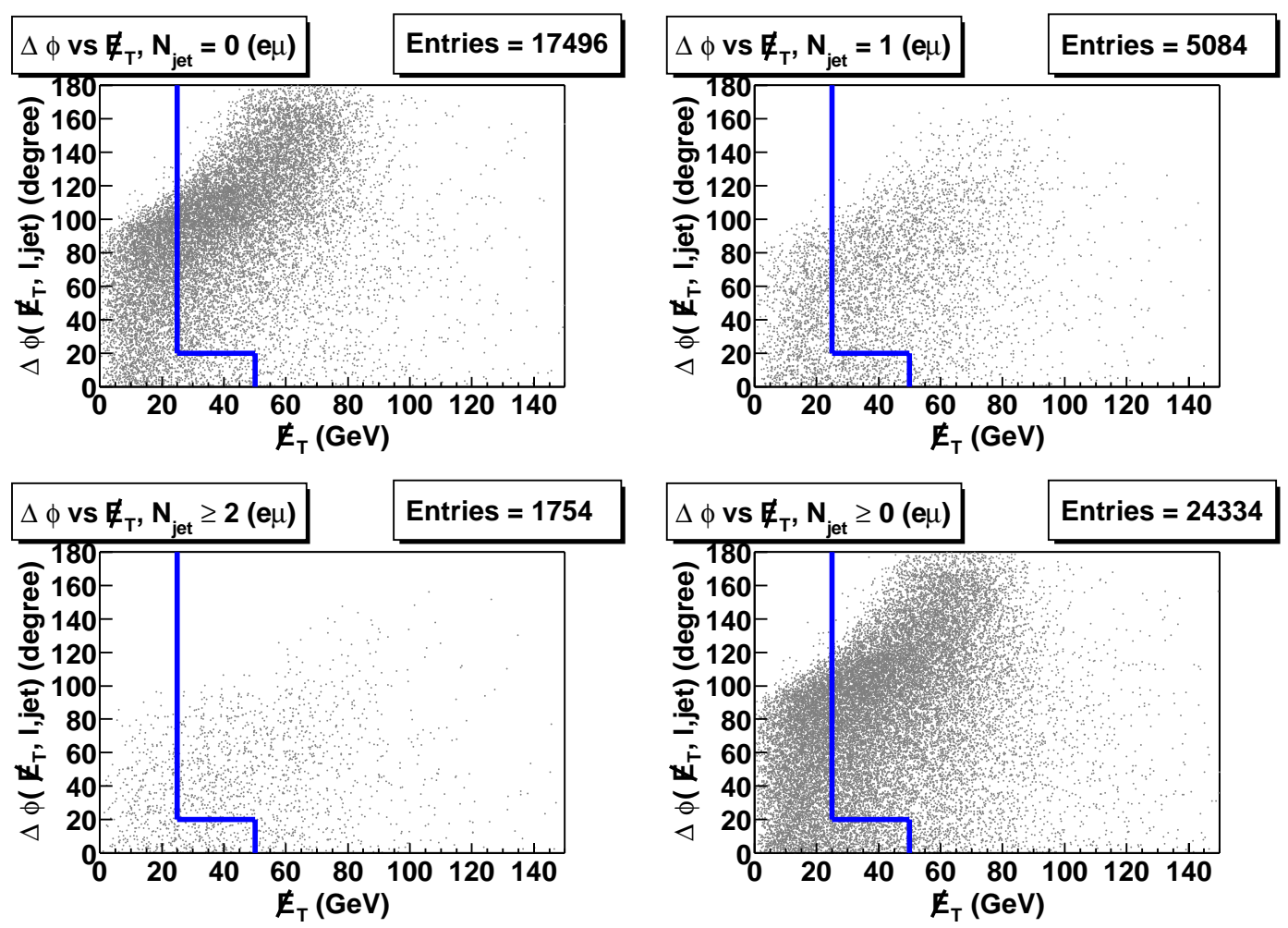

Figure 4.11: The magnitude of the missing $E_{T}\left(E_{T}, x\right.$ axis $)$ and the $\Delta \phi$ between $\mathbb{E}_{T}$ and the closest jet or lepton (y axis). $e \mu$ events in the $W W$ PYTHIA Monte Carlo sample are shown with no jet ( $N_{j}=0$, top left), $N_{j}=1$ (top right), $N_{j} \geq 2$ (bottom left), $N_{j} \geq 0$ (bottom right). The lines show the cut imposed for the $\mathbb{E}_{T}$ requirement. This sample is equivalent to $608.5 \mathrm{fb}^{-1}$. 

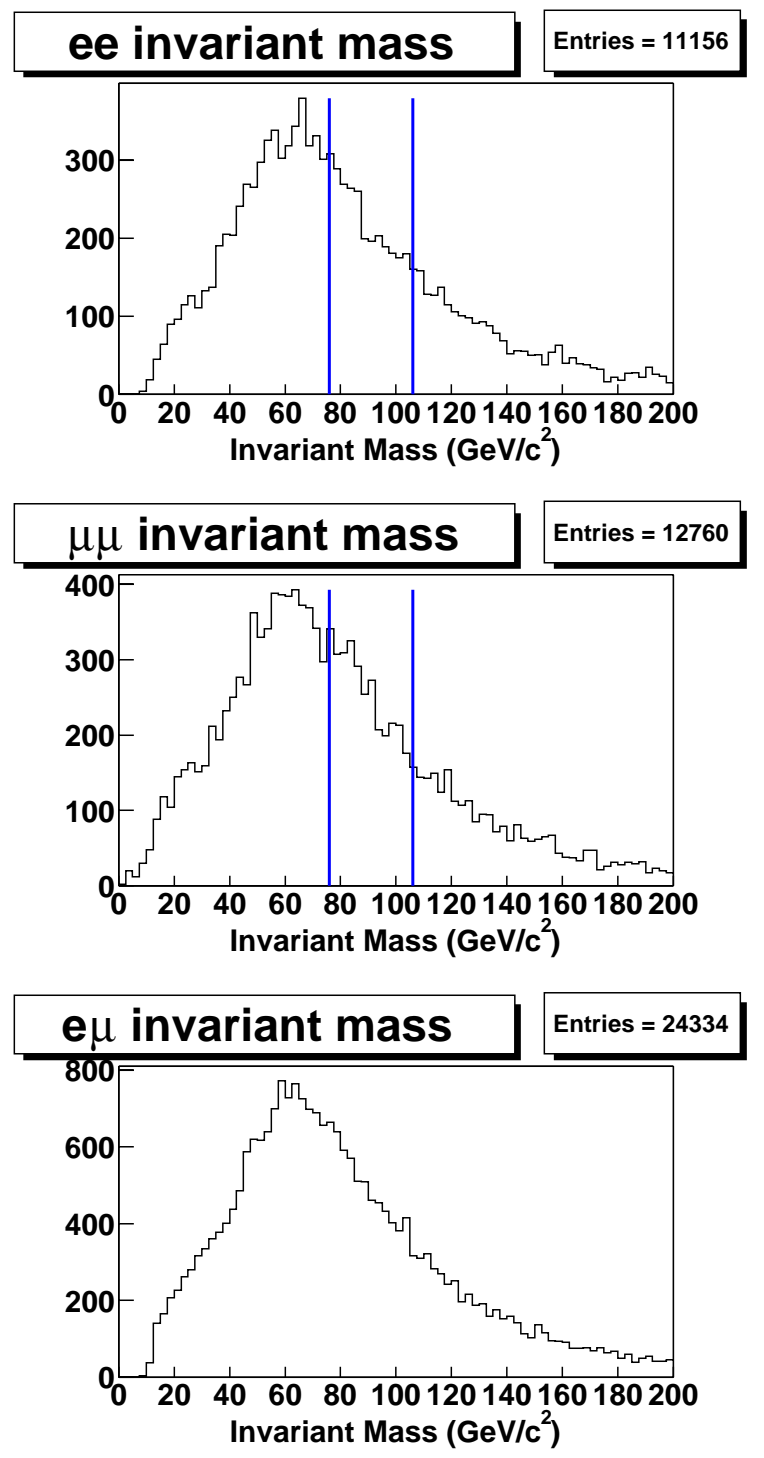

Figure 4.12: Invariant mass of two leptons $\left(m_{\ell \ell}\right)$ in the dilepton events of the PYTHIA $W W$ Monte Carlo sample after the isolation requirement imposed. Dielectron (ee, top), di-muon ( $\mu \mu$, middle), electron-muon $(e \mu$, bottom) events are shown separately. In the $e \mu$ category, the invariant mass cut is not imposed. The red lines in the $e e, e \mu$ show the invariant mass cut of $76 \mathrm{GeV} / \mathrm{c}^{2}<m_{\ell \ell}<106 \mathrm{GeV} / \mathrm{c}^{2}$. This sample is equivalent to $608.5 \mathrm{fb}^{-1}$. 

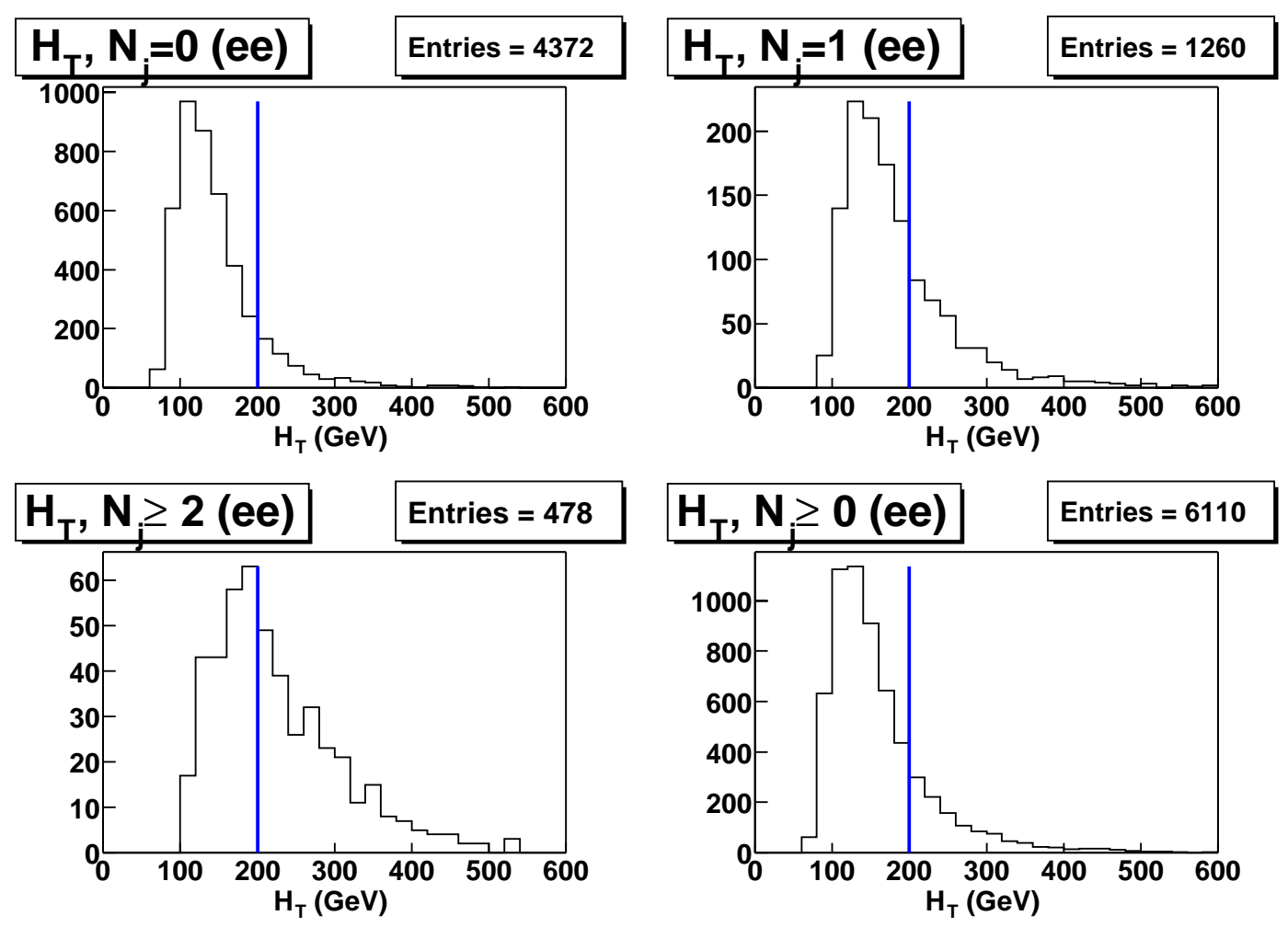

Figure 4.13: The $H_{T}$ of di-electron events after $E_{T}$ requirement in the $W W$ PYTHIA Monte Carlo sample, equivalent to $608.5 \mathrm{fb}^{-1}$. The plots are shown with no jet ( $N_{j}=0$, top left), $N_{j}=1$ (top right), $N_{j} \geq 2$ (bottom left), $N_{j} \geq 0$ (bottom right). The line shows the the cut for dilepton events $\left(H_{T}>200 \mathrm{GeV}\right)$. Only the events with $N_{j} \geq 2$ are selected for the final $t \bar{t}$ dilepton events. 

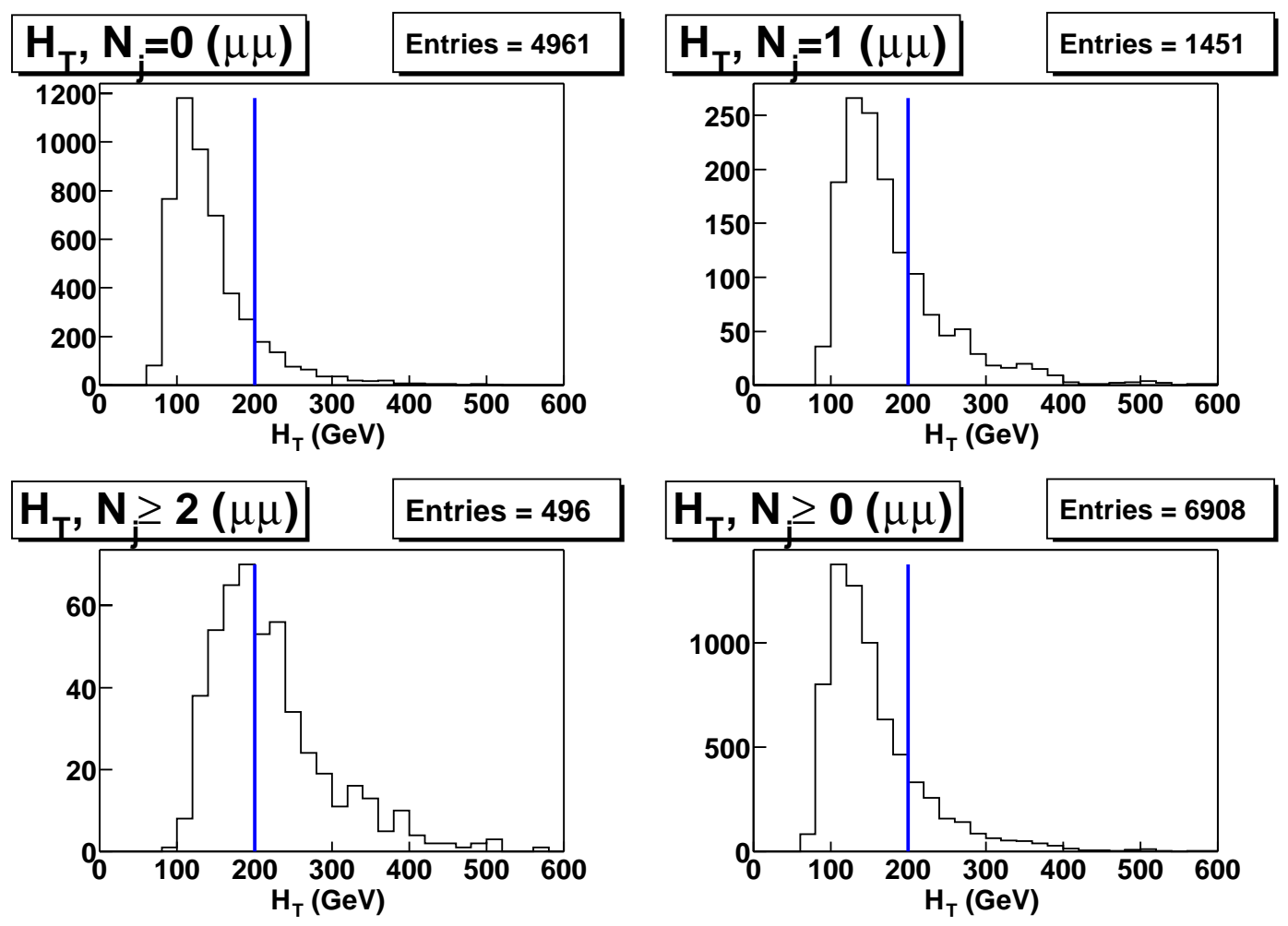

Figure 4.14: The $H_{T}$ of di-muon events after $\not_{T}$ requirement in the $W W$ PYTHIA Monte Carlo sample, equivalent to $608.5 \mathrm{fb}^{-1}$. The plots are shown with no jet ( $N_{j}=0$, top left), $N_{j}=1$ (top right), $N_{j} \geq 2$ (bottom left), $N_{j} \geq 0$ (bottom right). The line shows the the cut for dilepton events $\left(H_{T}>200 \mathrm{GeV}\right)$. Only the events with $N_{j} \geq 2$ are selected for the final $t \bar{t}$ dilepton events. 

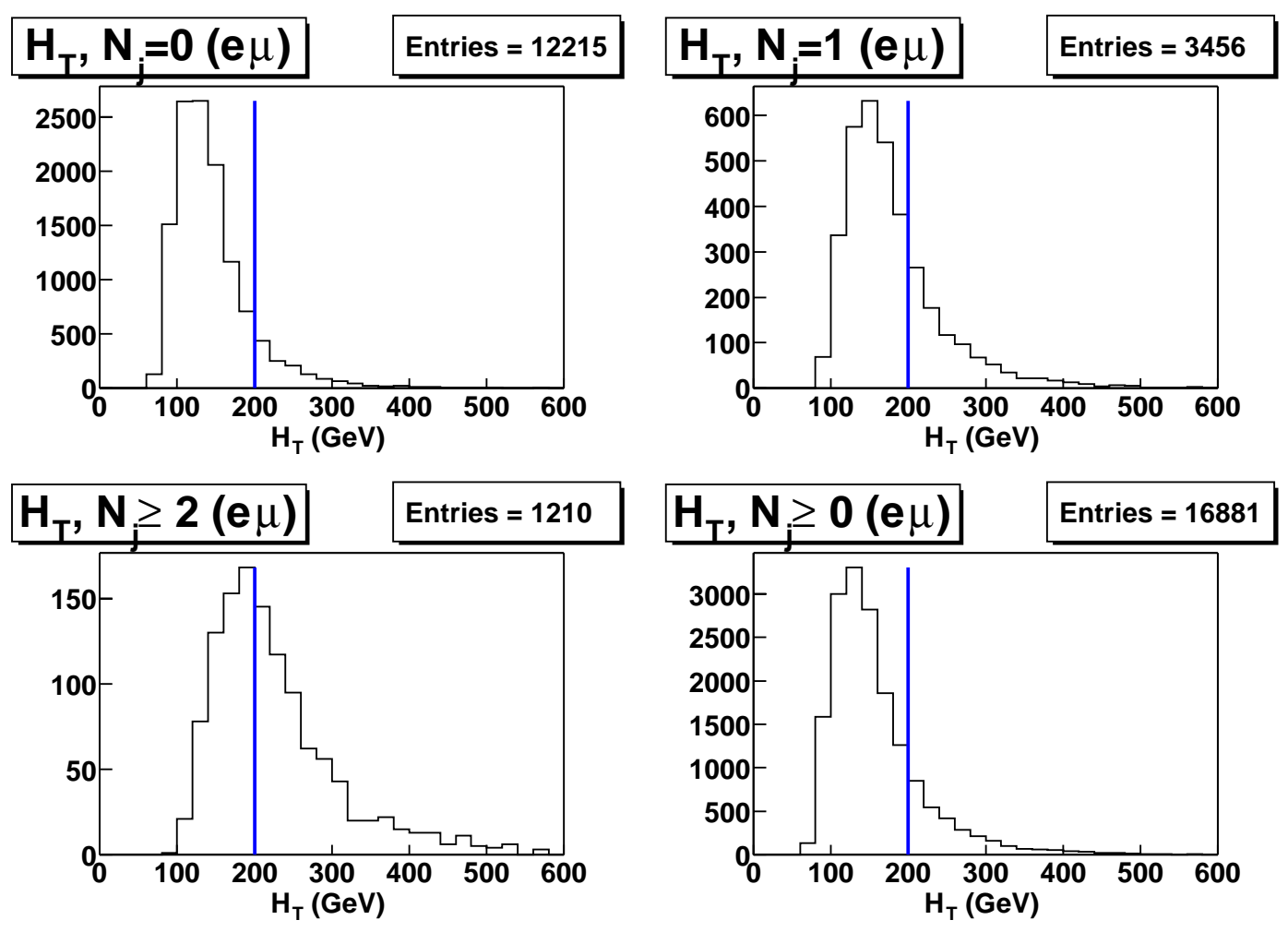

Figure 4.15: The $H_{T}$ of $e-\mu$ events after $H_{T}$ requirement in the $W W$ PYTHIA Monte Carlo sample, equivalent to $608.5 \mathrm{fb}^{-1}$. The plots are shown with no jet $\left(N_{j}=0\right.$, top left), $N_{j}=1$ (top right), $N_{j} \geq 2$ (bottom left), $N_{j} \geq 0$ (bottom right). The line shows the the cut for dilepton events $\left(H_{T}>200 \mathrm{GeV}\right)$. Only the events with $N_{j} \geq 2$ are selected for the final $t \bar{t}$ dilepton events. 
subset of events is clearly not in the signal sample. In the next step, we need the ratio of jets faking leptons (satisfying all the lepton identification requirements) to the jets reconstructed as leptons but failing some of the lepton identification requirements. By applying this ratio to the number of $W+\geq 3$ jet events obtained above (with one lepton failing the lepton ID), we can estimate the number of $W+\geq 3$ jet events where both of leptons satisfy the lepton ID but one of them is caused by a jet (not from a real lepton). For convenience, the sample of jets that fake leptons is denoted as control sample 1. The sample of jets that are reconstructed as leptons but fail the lepton ID is denoted as control sample 2. The ratio of control sample 1 to control sample 2 is denoted as fake ratio. Note that this is not a fake efficiency in a conventional sense because they are mutually exclusive. The control sample 1 and control sample 2 are prepared using the jet 20 trigger sample. The assumption is that this sample is dominated by only jet production and do not include real leptons. Therefore, in these samples, the leptons reconstructed by the CDF offline software are assumed not to be actual leptons. The requirements used for preparing control sample 1 and control sample 2 are listed in Table 4.5. The counting result from these samples and the fake ratio are shown in Table 4.6.

The next step of the estimation of the fake lepton background is counting the $W+\geq 3$ jet events in the data sample. To apply the fake ratio obtained above, we count the number of $W+\geq 3$ jet events that have a lepton falling in the criteria of control sample 2. In other words, the required sample contains the events which have one high $p_{T}$ lepton, large $\not_{T}$, at least two jets and one lepton failing the lepton ID as shown in the control sample 2 criteria of Table 4.5. The remaining dilepton 


\begin{tabular}{|c|c|c|}
\hline & & Requirements \\
\hline \multirow[b]{2}{*}{ Electron } & $\begin{array}{c}\text { Control } \\
\text { sample } 1\end{array}$ & $\begin{array}{l}E_{T}>20 \mathrm{GeV}, p_{T}>10 \mathrm{GeV} / \mathrm{c} \\
\text { Lepton identification described in Section } 3.1 \\
\text { Iso4 }<0.1 \\
\text { Not from } W, Z^{0} \text {, photon conversion }\end{array}$ \\
\hline & $\begin{array}{c}\text { Control } \\
\text { sample } 2\end{array}$ & $\begin{array}{l}E_{T}>20 \mathrm{GeV}, p_{T}>10 \mathrm{GeV} / \mathrm{c} \\
\mathrm{Had} / \mathrm{Em}>0.055+0.00045 \times E \text { or } \\
\mathrm{Lshr}>0.2 \\
\text { No isolation requirement } \\
\text { Not from } W, Z^{0}, \text { photon conversion }\end{array}$ \\
\hline \multirow[b]{2}{*}{ Muon } & $\begin{array}{c}\text { Control } \\
\text { sample } 1\end{array}$ & $\begin{array}{l}p_{T}>20 \mathrm{GeV} / \mathrm{c} \\
\text { Lepton identification described in Section } 3.2 \\
I s o 4<0.1 \\
\text { Not from } W, Z^{0} \text { and cosmic }\end{array}$ \\
\hline & $\begin{array}{c}\text { Control } \\
\text { sample } 2\end{array}$ & $\begin{array}{l}p_{T}>20 \mathrm{GeV} / \mathrm{c} \\
E_{E M}>\max (2.0,2.0+0.0115 \times(p-100)) \mathrm{GeV} \\
E_{H A D}>\max (6.0,6.0+0.028 \times(p-100)) \mathrm{GeV} \\
d_{0} \text { as described in Section } 3.2 \\
\text { No isolation requirement } \\
\text { Not from } W, Z^{0} \text { and cosmic }\end{array}$ \\
\hline
\end{tabular}

Table 4.5: The requirements of the control sample 1 and control sample 2 for the fake ratio calculation. 


\begin{tabular}{c|ccc}
\hline & Control & Control & \\
& sample 1 & sample 2 & Fake ratio \\
\hline \hline TCE & 72 & 3749 & $0.019 \pm 0.002$ \\
CMU & 2 & 259 & $0.008 \pm 0.005$ \\
CMP & 5 & 26 & $0.192 \pm 0.077$ \\
CMUP & 5 & 124 & $0.04 \pm 0.018$ \\
CMX & 0 & 59 & 0 \\
\hline
\end{tabular}

Table 4.6: The result of counting the control sample 1 and control sample 2 from the jet 20 sample.

\begin{tabular}{c|cc}
\hline & $\begin{array}{c}N_{W+\geq 3 j e t} \\
\text { before the } H_{T}, \\
\text { opposite sign }\end{array}$ & $\begin{array}{c}N_{b g}^{\text {fake }} \\
\left(109.4 \mathrm{pb}^{-1}\right)\end{array}$ \\
\hline \hline TCE TCE & 10 & $0.192 \pm 0.061$ \\
TCE CMUP & 4 & $0.077 \pm 0.038$ \\
TCE CMU & 1 & $0.008 \pm 0.008$ \\
TCE CMP & 1 & $0.019 \pm 0.019$ \\
TCE CMX & 2 & $0.038 \pm 0.027$ \\
\hline
\end{tabular}

Table 4.7: The result of counting the $W+\geq 3$ jet events with one lepton of control sample 2 type before the $H_{T}$ and the opposite sign requirement applied in the data. The fake ratio of each lepton type is applied to these numbers to give the expected backgrounds from the fake lepton in $109 \mathrm{pb}^{-1}$. 


\begin{tabular}{c|cc}
\hline & $\begin{array}{c}N_{W+\geq 3 j e t} \\
\text { after the } H_{T}, \\
\text { before opposite sign }\end{array}$ & $\begin{array}{c}N_{b g}^{\text {fake }} \\
\left(109.4 \mathrm{pb}^{-1}\right)\end{array}$ \\
\hline \hline TCE TCE & 7 & $0.134 \pm 0.051$ \\
TCE CMUP & 4 & $0.077 \pm 0.038$ \\
TCE CMU & 1 & $0.008 \pm 0.008$ \\
TCE CMP & 1 & $0.019 \pm 0.019$ \\
TCE CMX & 0 & - \\
\hline
\end{tabular}

Table 4.8: The result of counting the $W+\geq 3$ jet events with one lepton of control sample 2 type after the $H_{T}$ and before the opposite sign requirement applied in the data. The fake ratio of each lepton type is applied to these numbers to give the expected backgrounds from the fake lepton in $109 \mathrm{pb}^{-1}$.

\begin{tabular}{c|cc}
\hline & $\begin{array}{c}N_{W+\geq 3 j e t} \\
\text { passing all dilepton cuts }\end{array}$ & $\begin{array}{c}N_{b g}^{\text {fake }} \\
\left(109.4 \mathrm{pb}^{-1}\right)\end{array}$ \\
\hline \hline TCE TCE & 5 & $0.096 \pm 0.043$ \\
TCE CMUP & 3 & $0.058 \pm 0.033$ \\
TCE CMU & 1 & $0.008 \pm 0.008$ \\
TCE CMP & 0 & - \\
TCE CMX & 0 & - \\
\hline
\end{tabular}

Table 4.9: The result of counting the $W+\geq 3$ jet events with one lepton of control sample 2 type after all the dilepton selection requirements applied in the data. The fake ratio of each lepton type is applied to these numbers to give the expected backgrounds from the fake lepton in $109 \mathrm{pb}^{-1}$. 
selection requirements $\left(m_{\ell \ell}, \mathbb{E}_{T}, H_{T}\right.$, opposite sign) are applied to this $W+\geq 3$ jet sample and the number of events surviving all the requirements is counted. This sample does not overlap with the dilepton signal sample which have both leptons satisfying the lepton ID. The results of counting the events in the $W+\geq 3$ jet sample are listed in Tables 4.7, 4.8 and 4.9 for the different stages of the dilepton cuts. The fake ratio of each lepton type is applied to the number of events containing the corresponding type of the lepton to give the expected number of backgrounds in $109 p b^{-1}$ of;

$$
N_{b g}^{f a k e}=0.16 \pm 0.05
$$

\subsection{Summary of the Backgrounds}

The backgrounds for the $t \bar{t}$ dilepton channel are summarized in Table 4.10. The table shows the results of the background estimation separately for each dilepton category of the $e e, \mu \mu, e \mu$. In the $\mu \mu$ category, the background from the fake lepton is not included because their contribution is extremely small as estimated in Section 4.4. The total background expectation for $109 p b^{-1}$ of the integrated luminosity in the $t \bar{t}$ dilepton channel is;

$$
N_{b g}^{e x p}=0.65 \pm 0.13 \text { events. }
$$

Table 4.10 also shows the expected $t \bar{t}$ events in $109 p b^{-1}$ for comparison. With this number of expected $t \bar{t}$ events, the signal to background ratio is 4.9 . 


\begin{tabular}{|c|c|c|c|c|c|c|}
\hline & $\begin{array}{c}\text { Before } \\
2 \text { jet cut } \\
\end{array}$ & 0 jet & 1 jet & $\geq 2$ jets & $H_{T}$ & $\begin{array}{c}\text { Opposite } \\
\text { sign }\end{array}$ \\
\hline \multicolumn{7}{|l|}{$(e e)$} \\
\hline$W W$ & $1.10 \pm 0.01$ & $0.79 \pm 0.05$ & $0.23 \pm 0.03$ & $0.09 \pm 0.02$ & $0.05 \pm 0.01$ & $0.05 \pm 0.01$ \\
\hline$W Z^{0}$ & $0.17 \pm 0.01$ & $0.08 \pm 0.01$ & $0.06 \pm 0.005$ & $0.02 \pm 0.01$ & $0.02 \pm 0.01$ & $0.01 \pm 0.003$ \\
\hline$Z^{0} \rightarrow \tau^{+} \tau^{-}$ & $0.12 \pm 0.04$ & $0.02 \pm 0.004$ & $0.06 \pm 0.02$ & $0.05 \pm 0.02$ & $0.03 \pm 0.01$ & $0.03 \pm 0.01$ \\
\hline Drell-Yan & $0.09 \pm 0.13$ & $0.09 \pm 0.13$ & - & - & - & - \\
\hline Fake lepton & $0.58 \pm 0.11$ & $0.21 \pm 0.06$ & $0.17 \pm 0.06$ & $0.19 \pm 0.06$ & $0.13 \pm 0.05$ & $0.10 \pm 0.04$ \\
\hline Total bg & $2.06 \pm 0.18$ & $1.19 \pm 0.16$ & $0.52 \pm 0.07$ & $0.35 \pm 0.07$ & $0.22 \pm 0.05$ & $0.18 \pm 0.05$ \\
\hline$t \bar{t}$ & $0.76 \pm 0.08$ & $0.01 \pm 0.004$ & $0.10 \pm 0.02$ & $0.65 \pm 0.07$ & $0.63 \pm 0.07$ & $0.62 \pm 0.07$ \\
\hline \multicolumn{7}{|l|}{$(\mu \mu)$} \\
\hline$W W$ & $1.24 \pm 0.01$ & $0.89 \pm 0.07$ & $0.26 \pm 0.04$ & $0.09 \pm 0.03$ & $0.05 \pm 0.02$ & $0.05 \pm 0.02$ \\
\hline$W Z^{0}$ & $0.19 \pm 0.01$ & $0.12 \pm 0.01$ & $0.04 \pm 0.004$ & $0.02 \pm 0.01$ & $0.01 \pm 0.005$ & $0.01 \pm 0.003$ \\
\hline$Z^{0} \rightarrow \tau^{+} \tau^{-}$ & $0.14 \pm 0.05$ & $0.01 \pm 0.004$ & $0.07 \pm 0.02$ & $0.06 \pm 0.03$ & $0.04 \pm 0.01$ & $0.04 \pm 0.01$ \\
\hline Drell-Yan & $0.23 \pm 0.19$ & $0.01 \pm 0.01$ & $0.01 \pm 0.01$ & $0.21 \pm 0.17$ & $0.11 \pm 0.09$ & $0.11 \pm 0.09$ \\
\hline Fake lepton & - & - & - & - & - & - \\
\hline Total bg & $1.80 \pm 0.20$ & $1.04 \pm 0.07$ & $0.38 \pm 0.05$ & $0.38 \pm 0.17$ & $0.20 \pm 0.09$ & $0.20 \pm 0.09$ \\
\hline$t \bar{t}$ & $0.76 \pm 0.08$ & - & $0.08 \pm 0.02$ & $0.68 \pm 0.08$ & $0.65 \pm 0.08$ & $0.65 \pm 0.08$ \\
\hline \multicolumn{7}{|l|}{$(e \mu)$} \\
\hline$W W$ & $3.03 \pm 0.02$ & $2.20 \pm 0.15$ & $0.62 \pm 0.08$ & $0.22 \pm 0.07$ & $0.12 \pm 0.03$ & $0.12 \pm 0.03$ \\
\hline$W Z^{0}$ & $0.37 \pm 0.01$ & $0.20 \pm 0.02$ & $0.12 \pm 0.01$ & $0.05 \pm 0.01$ & $0.03 \pm 0.01$ & $0.02 \pm 0.01$ \\
\hline$Z^{0} \rightarrow \tau^{+} \tau^{-}$ & $0.32 \pm 0.10$ & $0.05 \pm 0.01$ & $0.15 \pm 0.05$ & $0.12 \pm 0.06$ & $0.06 \pm 0.03$ & $0.06 \pm 0.03$ \\
\hline Fake lepton & $0.66 \pm 0.11$ & $0.29 \pm 0.07$ & $0.23 \pm 0.07$ & $0.14 \pm 0.05$ & $0.10 \pm 0.04$ & $0.07 \pm 0.03$ \\
\hline Total bg & $4.38 \pm 0.10$ & $2.73 \pm 0.17$ & $1.12 \pm 0.12$ & $0.53 \pm 0.11$ & $0.32 \pm 0.06$ & $0.27 \pm 0.06$ \\
\hline$t \bar{t}$ & $2.21 \pm 0.22$ & $0.01 \pm 0.006$ & $0.23 \pm 0.05$ & $1.97 \pm 0.21$ & $1.92 \pm 0.20$ & $1.91 \pm 0.20$ \\
\hline \multicolumn{7}{|l|}{$(\ell \ell)$} \\
\hline$W W$ & $5.38 \pm 0.03$ & $3.87 \pm 0.28$ & $1.11 \pm 0.15$ & $0.39 \pm 0.13$ & $0.21 \pm 0.06$ & $0.21 \pm 0.06$ \\
\hline$W Z^{0}$ & $0.72 \pm 0.02$ & $0.40 \pm 0.03$ & $0.22 \pm 0.01$ & $0.10 \pm 0.02$ & $0.06 \pm 0.02$ & $0.04 \pm 0.01$ \\
\hline$Z^{0} \rightarrow \tau^{+} \tau^{-}$ & $0.58 \pm 0.19$ & $0.08 \pm 0.01$ & $0.27 \pm 0.09$ & $0.23 \pm 0.11$ & $0.13 \pm 0.05$ & $0.13 \pm 0.05$ \\
\hline Drell-Yan & $0.33 \pm 0.23$ & $0.11 \pm 0.13$ & $0.01 \pm 0.01$ & $0.21 \pm 0.17$ & $0.11 \pm 0.09$ & $0.11 \pm 0.09$ \\
\hline Fake lepton & $1.24 \pm 0.15$ & $0.50 \pm 0.10$ & $0.40 \pm 0.09$ & $0.33 \pm 0.08$ & $0.24 \pm 0.07$ & $0.16 \pm 0.05$ \\
\hline Total bg & $8.24 \pm 0.34$ & $4.96 \pm 0.32$ & $2.02 \pm 0.20$ & $1.26 \pm 0.25$ & $0.74 \pm 0.14$ & $0.65 \pm 0.13$ \\
\hline$t \bar{t}$ & $3.74 \pm 0.36$ & $0.02 \pm 0.01$ & $0.41 \pm 0.08$ & $3.31 \pm 0.34$ & $3.20 \pm 0.34$ & $3.18 \pm 0.34$ \\
\hline
\end{tabular}

Table 4.10: The background contributions to the dilepton channels in $109 p b^{-1}$ of integrated luminosity. The contributions from 0 jet and 1 jet bin are separately shown before the $H_{T}$ is applied. The expected number of events from $t \bar{t}$ production using a PYTHIA Monte Carlo sample with $m_{t}=175 \mathrm{GeV} / \mathrm{c}^{2}$ are also shown. 


\section{CHAPTER 5}

\section{RESULTS}

\subsection{Data sample}

The CDF detector has observed a huge number of $p \bar{p}$ collisions. It is impossible to record every collision. As described in Section 2.2.4, the triggers selectively pass the interesting physics events to the permanent storage and therefore make the samples a manageable size. For the dilepton analysis, two different data samples are prepared depending on the triggers and the lepton types. The events which contain at least one high $p_{T}$ central electron are collected in the datasets bhelos and bhel09. The events in these datasets are triggered mainly by the ELECTRON_CENTRAL_18 trigger path.

The events in bhel08, bhel09 are filtered again with more stringent cuts in the offline stage. These cuts are listed in Table 5.1. These cuts are looser than the lepton identification cuts used for the final dilepton selection. The sample made from datasets bhel08 and bhel09 with the cuts in Table 5.1 is called the inclusive electron sample [53].

The dataset for the events with muons are prepared in a similar manner. The datasets bhmu08 and bhmu09 consist of the events triggered by the MUON_CMUP18 


\begin{tabular}{c}
\hline Cuts \\
\hline \hline \\
$E_{T} \geq 18 \mathrm{GeV}$ \\
$E_{\text {had }} / E_{\text {em }} \leq 0.125$ \\
$L s h r \leq 0.3$ \\
$p_{T} \geq 9.0 \mathrm{GeV} / \mathrm{c}$ \\
$E / p \leq 4.0$ \\
$|\Delta x| \leq 3.0 \mathrm{~cm}$ \\
$|\Delta z| \leq 5.0 \mathrm{~cm}$ \\
\end{tabular}

Table 5.1: The selection cuts for the electrons in the inclusive electron sample. At least one electron passing these cuts is required for the event to be included in the inclusive electron sample. This sample is used for the dilepton analysis.

and MUON_CMX18 trigger paths. The inclusive muon [53] dataset is prepared with additional cuts on muons as listed in Table 5.2. The data samples used in this analysis were collected from August 2002 (run number 150145) to May 2003 (run number 163527). The integrated luminosity of the data in this period is $\mathcal{L}=$ $109.4 \pm 6.4 p b^{-1}$.

\subsection{The $t \bar{t}$ dilepton candidate events}

The dilepton selection cuts were applied to the data sample of $109 p b^{-1}$ described in the previous section. In Table 5.3, the number of events passing the dilepton 


\begin{tabular}{c}
\hline Cuts \\
$p_{T} \geq 18 \mathrm{GeV} / \mathrm{c}$ \\
$E_{\text {em }} \leq(3+0.0140 \times(p-100)) \mathrm{GeV}$ \\
$\left(E_{\text {em }} \leq 3 \mathrm{GeV}\right.$ when $\left.p<100 \mathrm{GeV} / \mathrm{c}\right)$ \\
$E_{\text {had }} \leq(9+0.0420 \times(p-100)) \mathrm{GeV}$ \\
$\left(E_{\text {had }} \leq 9 \mathrm{GeV}\right.$ when $\left.p<100 \mathrm{GeV} / \mathrm{c}\right)$ \\
$|\Delta x|_{C M U} \leq 5.0 \mathrm{~cm}$ \\
$|\Delta x|_{C M P} \leq 10.0 \mathrm{~cm}$ \\
$|\Delta x|_{C M X} \leq 20.0 \mathrm{~cm}$ \\
\end{tabular}

Table 5.2: The selection cuts for the muons in the inclusive muon sample. At least one muon passing these cuts is required for the event to be part of the inclusive muon sample. This sample is used for the dilepton analysis. 
selection cuts are shown for various dilepton categories. After all the selection cuts, 1 ee event, $3 \mu \mu$ events and $2 e \mu$ events survive. The kinematic properties of the six $t \bar{t}$ candidate events are listed in Table 5.4.

The distribution of the $H_{T}$ defined in Section 3.3.5 is shown in Figure 5.1 for the six $t \bar{t}$ candidate events. For comparison, the $H_{T}$ distribution of the PYTHIA $t \bar{t}$ Monte Carlo sample is given along with the candidate events. The events of the $t \bar{t}$ Monte Carlo sample shown in Figure 5.1 are the events which have passed all the dilepton selection cuts except the $H_{T}$ cut. Also, the $H_{T}$ distribution of the backgrounds from $W W$ and $Z^{0} \rightarrow \tau^{+} \tau^{-}$Monte Carlo samples is shown. The relative contributions from the background and the $t \bar{t}$ distribution are adjusted to the expected proportions estimated in Chapter 3 and 4 . The distributions are normalized to 9 times the expected numbers so that shapes can be easily compared with the dilepton candidates from data.

The invariant mass of two leptons is plotted in Figure 5.2. The distribution from the $t \bar{t}$ Monte Carlo sample which has passed all the dilepton selection cuts except the invariant mass cut is shown for comparison. The $t \bar{t}$ distribution is normalized to 20 times the expected number for the same reason as above. The candidate events appear mostly in the lower side of the mass cut. In Figure 5.3, the $\mathbb{E}_{T}$ versus $\Delta \phi$ is plotted for the six candidate events and the $t \bar{t}$ Monte Carlo sample. Recall that $\Delta \phi$ is the angle in the transverse plane between $\mathbb{E}_{T}$ and the nearest lepton or jet as defined in Section 3.3.3. The distribution of the $t \bar{t}$ sample is obtained from the events which pass all the dilepton selection cuts except the $\not_{T}$ cut. 


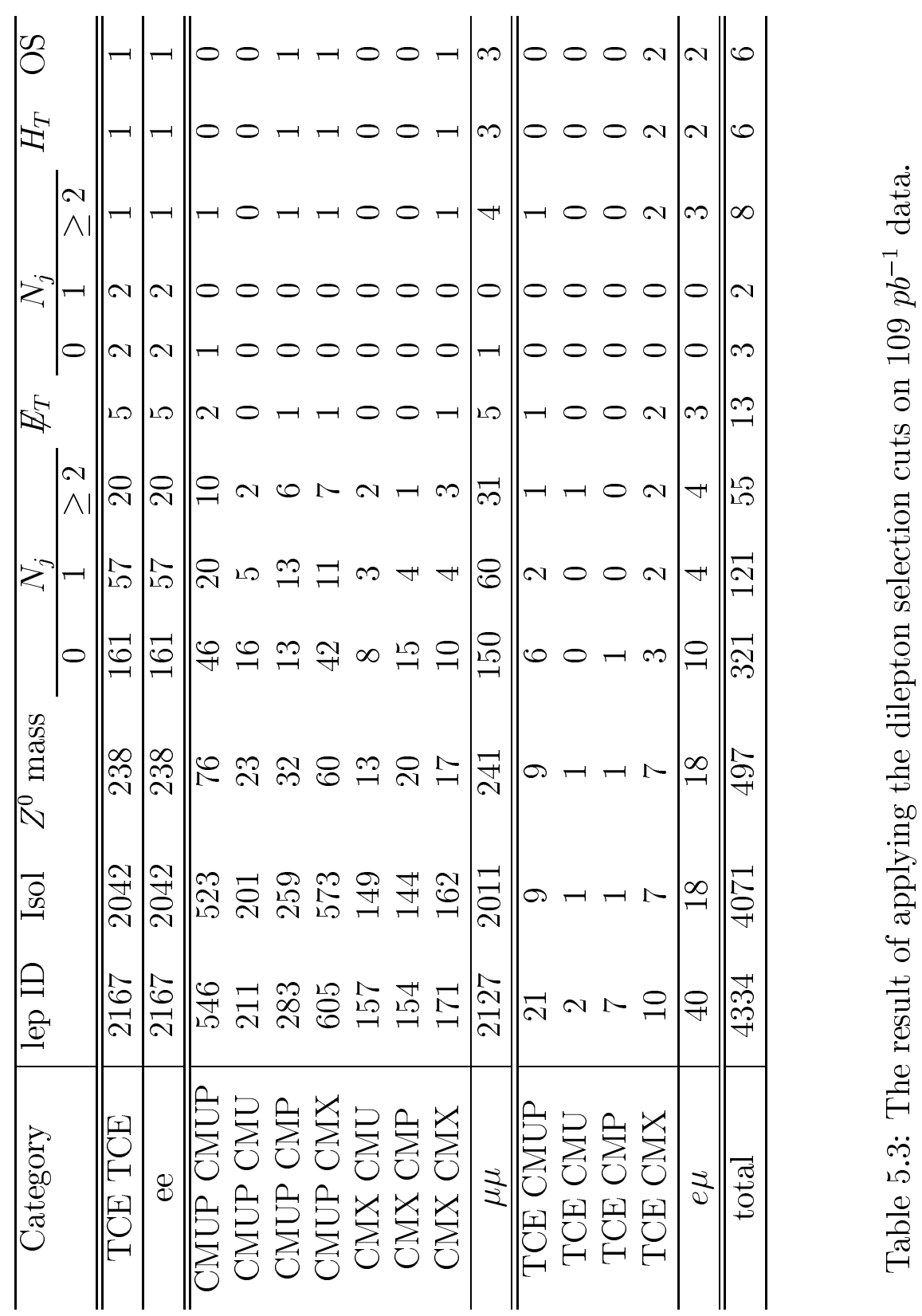




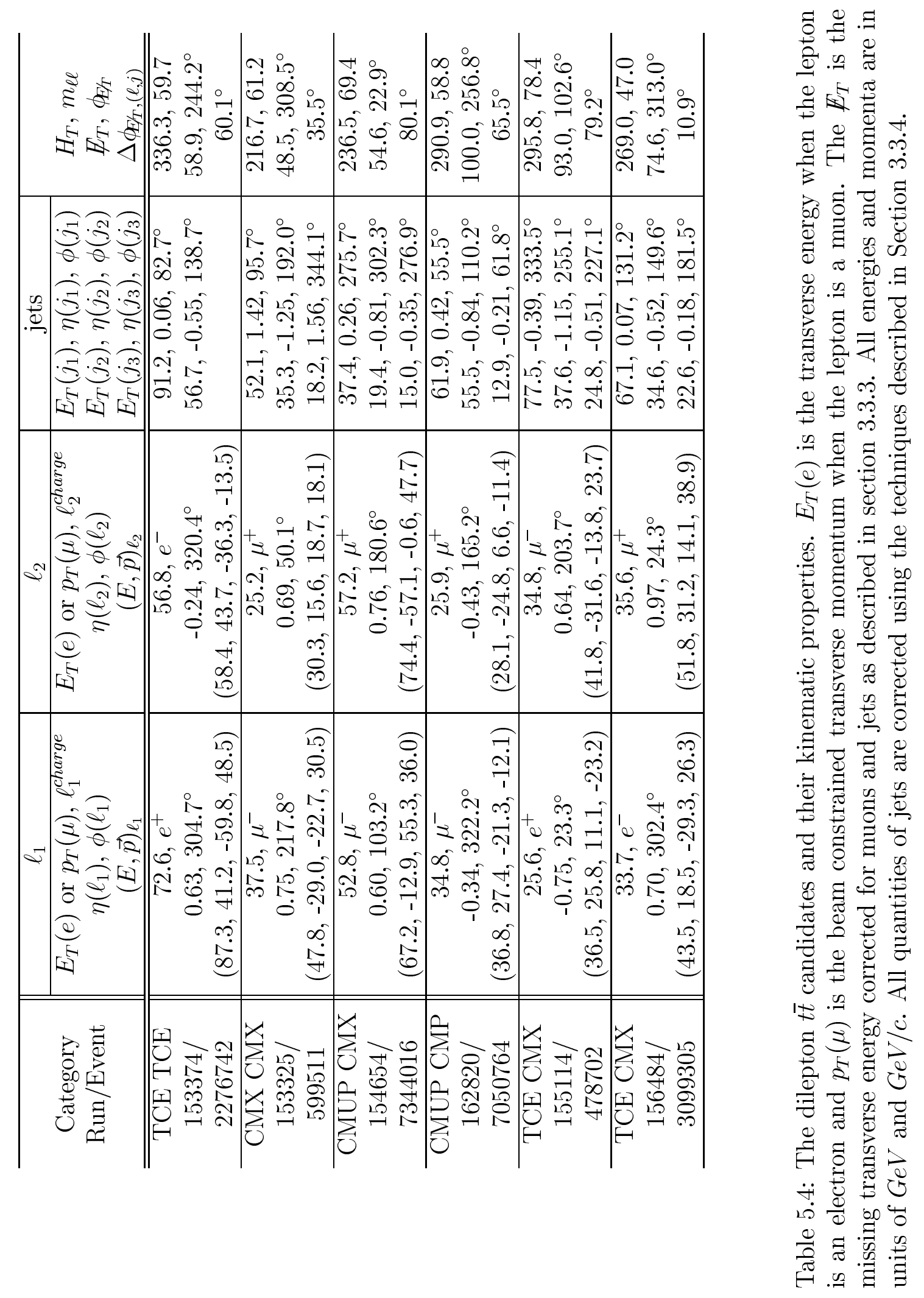




\subsection{The $t \bar{t}$ cross section}

The cross section for $t \bar{t}$ production is calculated by Equation 5.1. All the numbers required for this calculation have been obtained in the previous chapters.

$$
\sigma_{t \bar{t}}=\frac{N_{o b s}-N_{b g}^{e x p}}{\epsilon_{d i l} \mathcal{L}}
$$

- $N_{o b s}=6$, the number of observed events,

- $N_{b g}^{e x p}=0.65 \pm 0.13$, expected number of background events (Chapter 4).

- $\epsilon_{d i l}=0.43 \pm 0.04 \%$, total $t \bar{t}$ dilepton channel acceptance (Chapter 3 ).

- $\mathcal{L}=109.4 \pm 6.4 p b^{-1}$, total integrated luminosity (Chapter 5).

In order to precisely calculate the uncertainty of the cross section measurement, the following likelihood function $L$ is introduced.

$$
L=e^{-\frac{(\mathcal{L}-\overline{\mathcal{L}})^{2}}{2 \sigma_{\mathcal{L}}^{2}}} e^{-\frac{\left(\epsilon_{d i l}-\overline{\epsilon_{d i l}}\right)^{2}}{2 \sigma_{\epsilon_{d i l}}^{2}}} e^{-\frac{\left(N_{b g}-\overline{N_{b g}}\right)^{2}}{2 \sigma_{N_{b g}}^{2}}} \frac{\left(\epsilon_{d i l} \sigma_{t \bar{t}} \mathcal{L}+N_{b g}\right)^{N_{o b s}}}{N_{o b s} !} e^{-\left(\epsilon_{d i l} \sigma_{t \bar{t}} \mathcal{L}+N_{b g}\right)}
$$

This is the Poisson probability of observing $N_{o b s}$ events when $\left(\epsilon_{d i l} \sigma_{t \bar{t}} \mathcal{L}+N_{b g}\right)$ events are expected. The number of expected events is smeared by the Gaussian distributions for $\mathcal{L}, \epsilon_{d i l}$ and $N_{b g}$ respectively. The $\overline{\mathcal{L}}, \overline{\epsilon_{d i l}}, \overline{N_{b g}}$ are taken from the numbers in the text as the mean values of the Gaussian distributions. The Gaussian errors $\sigma_{\mathcal{L}}, \sigma_{\epsilon_{d i l}}$ and $\sigma_{N_{b g}}$ are also taken from the numbers in the text as the errors of the Gaussian distributions.

The $\mathcal{L}, \epsilon_{d i l}, N_{b g}$ and $\sigma_{t \bar{t}}$ are allowed to vary to obtain the minimum of $-\ln L$. At this minimum point of $-\ln L$, the cross section is obtained from the value of 


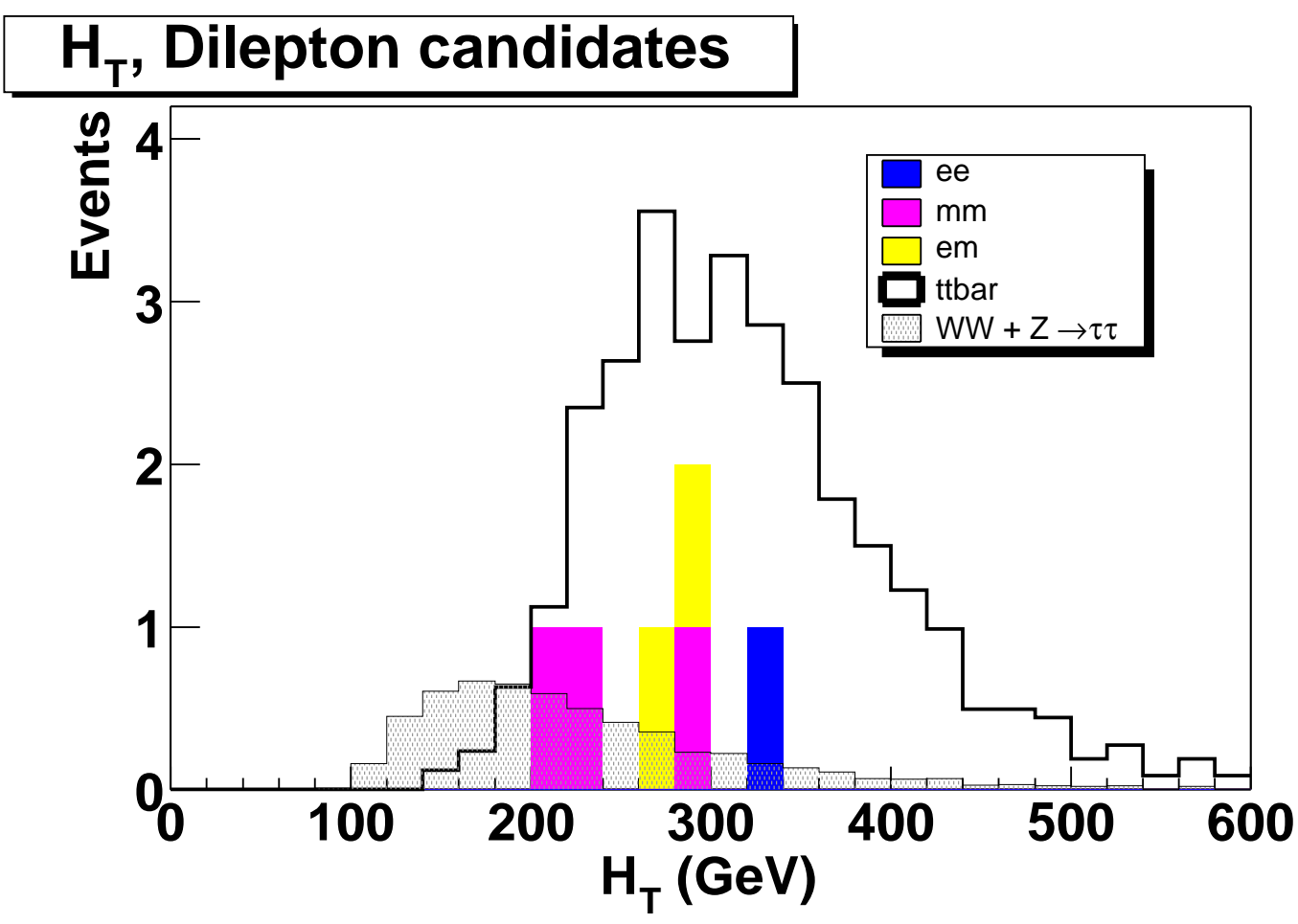

Figure 5.1: The $H_{T}$ distributions of the six dilepton candidates, the expected backgrounds from $W W, Z^{0} \rightarrow \tau^{+} \tau^{-}$and $t \bar{t}$ Monte Carlo sample with $m_{t}=175 \mathrm{GeV} / \mathrm{c}^{2}$. The number of events for the backgrounds and $t \bar{t}$ Monte Carlo are normalized to 9 times the expected numbers. 


\section{Dilepton invariant mass}

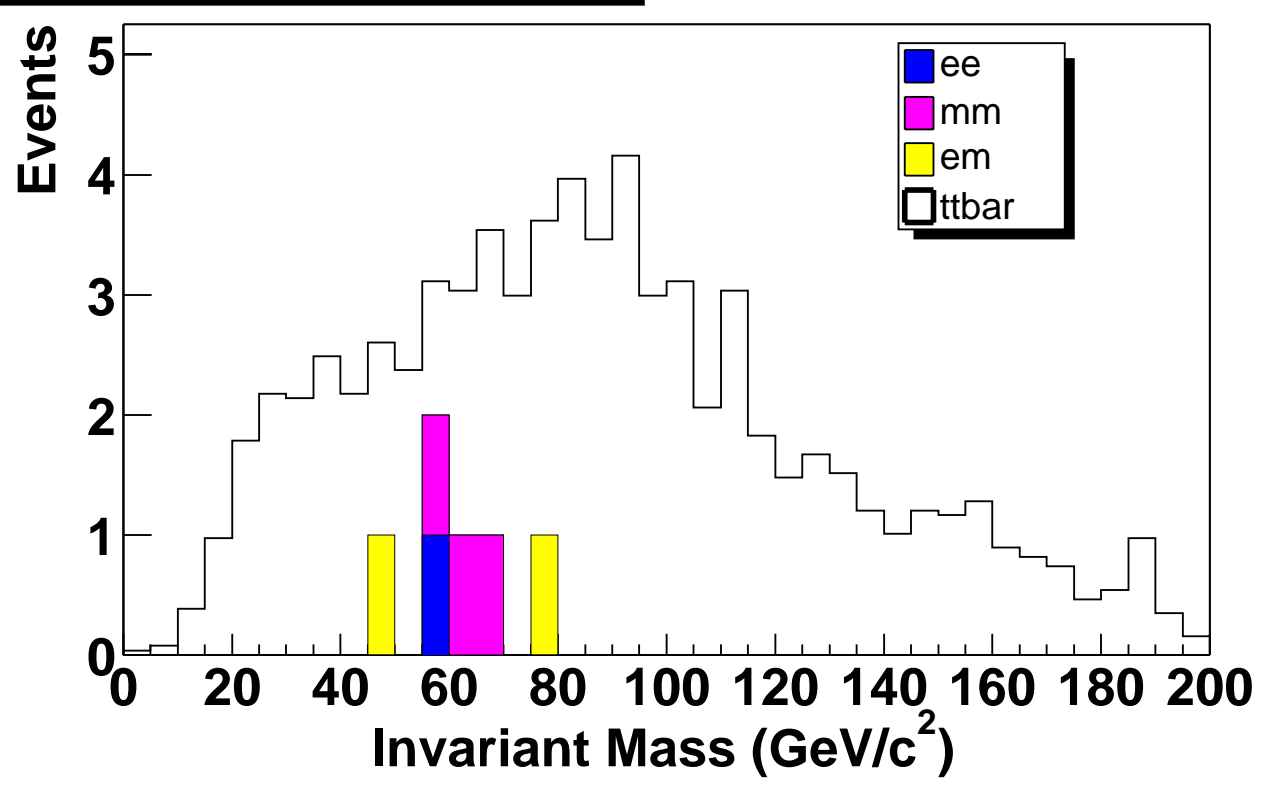

Figure 5.2: The dilepton invariant mass distribution $\left(m_{\ell \ell}\right)$ of the six dilepton candidates. The distribution from the $t \bar{t}$ Monte Carlo sample $\left(m_{t}=175 \mathrm{GeV} / \mathrm{c}^{2}\right)$ are also shown. The number of events for the $t \bar{t}$ Monte Carlo sample is normalized to 20 times the expected number. 


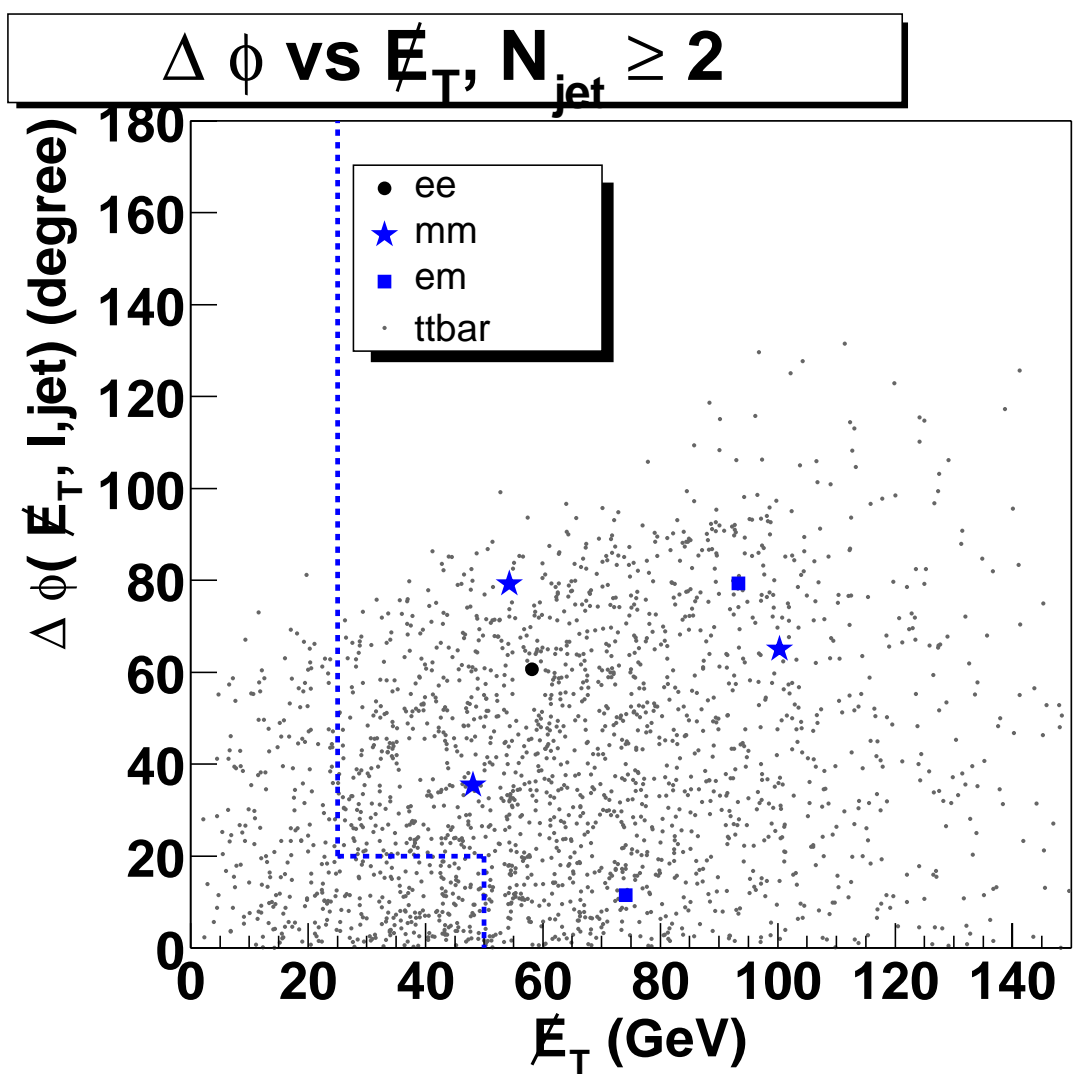

Figure 5.3: The $E_{T}$ versus $\Delta \phi$ is plotted for the six candidate events and the $t \bar{t}$ Monte Carlo sample $\left(m_{t}=175 \mathrm{GeV} / \mathrm{c}^{2}\right) . \Delta \phi$ is the angle in the transverse plane between $\mathscr{E}_{T}$ and the nearest lepton or jet. The dashed lines represent the $\not_{T}$ cuts. 
$\sigma_{t \bar{t}}$. However, this value is nothing but the value which can be trivially calculated from Equation 5.1. The Equation 5.2 is more useful for extracting the uncertainty on the cross section. Using this likelihood, the asymmetric uncertainty can be obtained at the points of $\Delta(-\ln L)=1 / 2$. Actual calculation of finding these points are performed by the CERNLIB MINUIT package [54]. The MINOS minimization algorithm is used. From this calculation, the result

$$
\sigma_{t \bar{t}}=11.4_{-4.6}^{+6.2} p b
$$

is obtained. The theoretical prediction of the $t \bar{t}$ production cross section from the NLO calculation by M. Cacciari et al., is $6.70{ }_{-0.88}^{+0.71} p b[16]$. 


\section{CHAPTER 6}

\section{CONCLUSION}

The $t \bar{t}$ production from the proton-antiproton collisions at $\sqrt{s}=1.96 \mathrm{TeV}$ has been observed in the dilepton decay channel. In a data sample of $109 p b^{-1}$, six candidate events were observed with an expected background of $0.65 \pm 0.13$. The $t \bar{t}$ production cross section in this channel is determined to be

$$
\sigma_{t \bar{t}}=11.4_{-4.6}^{+6.2} p b
$$

The measured cross section is consistent with the Standard Model prediction within about one standard deviation.

The top quark properties, including its mass, production cross section, branching fractions and various kinematic distributions, also will be measured in the large data samples being accumulated by the CDF detector over the next few years. The top quark is one of the fundamental particles of the Standard Model. Its large mass makes it an interesting particle to study. Investigating all its properties may yield new understanding of the Standard Model or even open up new paradigms. 


\section{APPENDIX A}

\section{LEPTON IDENTIFICATION EFFICIENCIES}

To calculate the lepton identification efficiency, $Z^{0} \rightarrow e^{+} e^{-}$and $Z^{0} \rightarrow \mu^{+} \mu^{-}$ events are used because they have very little background under the $Z^{0}$ peak. There are two methods for extracting the efficiency from these events. The first method looks for the tight lepton (the lepton passing all the lepton ID cuts) and another lepton which has a relatively loose requirement. For the tight central electron (TCE), the loose requirements are $E_{T}>20 \mathrm{GeV}, p_{T}>10 \mathrm{GeV} / c$, fiducial (FidEle $=4$ ), and $\left|z_{0}\right|<60 \mathrm{~cm}$. The two electrons are required to have $75 \mathrm{GeV} / \mathrm{c}^{2}<M_{e e}<$ $105 \mathrm{GeV} / \mathrm{c}^{2}$. Then, the second electron is checked to determine if it passes the tight electron identification requirements. This method has an advantage of high statistics but is limited to the same type of leptons to both legs of $Z^{0}$. The ID efficiency from this method [50] is given by

$$
\frac{2 N_{\mathcal{X X}}}{2 N_{\mathcal{X X}}+N_{\mathcal{X O}}}
$$

where $N_{\mathcal{X}}$ is the number of events with both legs passing tight ID cuts and $N_{\mathcal{X} \mathcal{O}}$ is the number of events when one leg passes but the other does not. 
For muon, there are several different types of muons with different ID variables. Therefore, an alternate approach is used to calculate the efficiency for this case. This method looks for two loose muons which have $p_{T}>20 \mathrm{GeV} / \mathrm{c},\left|z_{0}\right|<60 \mathrm{~cm}$, and $d 0$ satisfying the criteria in Table 3.3 as well as the following fiducial requirements.

- CMUP : stubs in the CMU and CMP both but not elsewhere.

- CMU only : a stub in the CMU only.

- CMP only : a stub in the CMP only.

- CMX : stubs in the CMX only.

If there are two loose muons, which satisfy $75 \mathrm{GeV} / \mathrm{c}^{2}<M_{\mu \mu}<105 \mathrm{GeV} / \mathrm{c}^{2}$, one of the muons is randomly chosen and checked if it passes all the muon ID requirements (CMUP or CMX are considered for this study as a tight leg). If passed, the number of events is counted as $N_{\text {total }}$. Among these events the number of events where the second muon also passes the ID cuts (for this leg, all muon types are considered) is counted as $N_{\text {pass }}$. The ID efficiency for this method is simply

$$
\frac{N_{\text {pass }}}{N_{\text {total }}}
$$

In this study, all leptons are required to be isolated $(I s o 4<0.1)$. The ID efficiencies of plug electrons are obtained in a similar way. Table A.1 to A.8 show the individual efficiencies that are obtained without any other cuts imposed and the $N-1$ efficiency that is obtained after all other cuts are imposed.

The ID efficiencies are also obtained from the PYTHIA $Z^{0} \rightarrow e^{+} e^{-}, \mu^{+} \mu^{-}$Monte Carlo samples. The efficiencies from these sample are compared to the efficiencies 


\begin{tabular}{c|cc}
\hline variable & individual & $\mathrm{N}-1$ \\
\hline \hline$E_{\text {had }} / E_{e m}$ & $0.9922 \pm 0.0016$ & $0.9924 \pm 0.0014$ \\
$\mathrm{E} / \mathrm{P}$ & $0.9170 \pm 0.0044$ & $0.9362 \pm 0.0040$ \\
$\mathrm{Lshr}$ & $0.9911 \pm 0.0015$ & $0.9947 \pm 0.0012$ \\
$Q \times \Delta x$ & $0.9831 \pm 0.0020$ & $0.9987 \pm 0.0006$ \\
$\Delta z$ & $0.9957 \pm 0.0011$ & $0.9990 \pm 0.0005$ \\
$\chi_{\text {strip }}^{2}$ & $0.9654 \pm 0.0028$ & $0.9699 \pm 0.0028$ \\
Track cut (\# SL) & $0.9721 \pm 0.0026$ & $0.9747 \pm 0.0025$ \\
\hline Total & $0.8506 \pm 0.0058$ & \\
\hline
\end{tabular}

Table A.1: The efficiency of the tight central electron (TCE) identification cuts using the $Z^{0} \rightarrow e^{+} e^{-}$data sample. All uncertainties are statistical.

from the $Z^{0}$ data sample to estimate the difference between the Monte Carlo detector simulation and the real detector. The ratios of efficiencies between the two sets of samples are applied to the $t \bar{t}$ acceptance calculation to give a more realistic value of $\epsilon_{d i l}$. The results from the PYTHIA $Z^{0} \rightarrow e^{+} e^{-}, \mu^{+} \mu^{-}$Monte Carlo samples are shown in Table A.9 to A.16. In Table A.17, the comparisons and the ratios between the efficiencies from the data and Monte Carlo samples are given. All categories show lower efficiencies in the data than the Monte Carlo, except the CMP. Figures A.1 to A.7 show the electron and muon identification variables for the data and the Monte Carlo samples. Most of the variables agree well between data and Monte Carlo except the PEM pesProfileRatio5by9. However these discrepancies do not cause a significant difference in the efficiency with the cut at 0.65 . 


\begin{tabular}{c|cc}
\hline variable & individual & $\mathrm{N}-1$ \\
\hline \hline$E_{\text {had }} / E_{\text {em }}$ & $0.9935 \pm 0.0017$ & $0.9932 \pm 0.0019$ \\
$\mathrm{PEM} \chi_{3 \times 3}^{2}$ & $0.9040 \pm 0.0063$ & $0.9162 \pm 0.0061$ \\
$\Delta R_{P E M-P E S}$ & $0.9838 \pm 0.0027$ & - \\
pesProfileRatio5by $9(\mathrm{U})$ & $0.9907 \pm 0.0021$ & $0.9942 \pm 0.0017$ \\
pesProfileRatio5by $9(\mathrm{~V})$ & $0.9907 \pm 0.0021$ & $0.9948 \pm 0.0016$ \\
\hline Total & $0.8822 \pm 0.0069$ & \\
\hline
\end{tabular}

Table A.2: The efficiency of the plug electron (PEM) identification cuts using the $Z^{0} \rightarrow e^{+} e^{-}$data sample. All uncertainties are statistical.

\begin{tabular}{c|cc}
\hline variable & individual & $\mathrm{N}-1$ \\
\hline \hline$E_{\text {had }} / E_{\text {em }}$ & $0.9935 \pm 0.0017$ & $0.9937 \pm 0.0026$ \\
PEM $\chi_{3 \times 3}^{2}$ & $0.9040 \pm 0.0063$ & $0.9206 \pm 0.0084$ \\
$\Delta R_{P E M-P E S}$ & $0.9838 \pm 0.0027$ & - \\
pesProfileRatio5by $9(\mathrm{U})$ & $0.9907 \pm 0.0021$ & $0.9948 \pm 0.0023$ \\
pesProfileRatio5by9 $(\mathrm{V})$ & $0.9907 \pm 0.0021$ & $0.9948 \pm 0.0023$ \\
\hline Phoenix Track matching & $0.5320 \pm 0.0107$ & - \\
Phoenix Silicon hits & $0.9276 \pm 0.0077$ & $0.9260 \pm 0.0082$ \\
Phoenix Track $z_{0}$ & $0.9974 \pm 0.0015$ & $0.9989 \pm 0.0010$ \\
\hline Total & $0.4411 \pm 0.0107$ & \\
\hline
\end{tabular}

Table A.3: The efficiencies of the Phoenix electron (PHX) identification cuts using the $Z^{0} \rightarrow e^{+} e^{-}$data sample. All uncertainties are statistical. The Phoenix Track matching cut is the efficiency that a PEM has a Phoenix track. The Phoenix Silicon hits, and Phoenix Track $z_{0}$ is obtained from the PEM that has a Phoenix track. The total efficiency is the probability that a PEM passes all PHX identification cuts and has a Phoenix track. 


\begin{tabular}{c|cc}
\hline variable & individual & $\mathrm{N}-1$ \\
\hline \hline em energy & $0.9573 \pm 0.0071$ & $0.9564 \pm 0.0075$ \\
had energy & $0.9829 \pm 0.0045$ & $0.9846 \pm 0.0046$ \\
$\Delta x_{c m u}$ & $0.9561 \pm 0.0072$ & $0.9551 \pm 0.0076$ \\
$\Delta x_{c m p}$ & $0.9829 \pm 0.0045$ & $0.9832 \pm 0.0048$ \\
Track cut (\# SL) & $0.9695 \pm 0.0060$ & $0.9669 \pm 0.0066$ \\
\hline Total & $0.8561 \pm 0.0123$ & \\
\hline
\end{tabular}

Table A.4: The efficiency of the central muon (CMUP) identification cuts using the $Z^{0} \rightarrow \mu^{+} \mu^{-}$data sample. All uncertainties are statistical.

\begin{tabular}{c|cc}
\hline variable & individual & $\mathrm{N}-1$ \\
\hline \hline em energy & $0.9583 \pm 0.0144$ & $0.9553 \pm 0.0154$ \\
had energy & $0.9792 \pm 0.0103$ & $0.9771 \pm 0.0113$ \\
$\Delta x_{\text {cmu }}$ & $0.9896 \pm 0.0073$ & $0.9884 \pm 0.0081$ \\
Track cut (\# SL) & $0.9635 \pm 0.0135$ & $0.9607 \pm 0.0146$ \\
\hline Total & $0.8906 \pm 0.0225$ & \\
\hline
\end{tabular}

Table A.5: The efficiency of the central muon (CMU only) identification cuts using the $Z^{0} \rightarrow \mu^{+} \mu^{-}$data sample. All uncertainties are statistical. 


\begin{tabular}{c|cc}
\hline variable & individual & $\mathrm{N}-1$ \\
\hline \hline em energy & $0.9754 \pm 0.0109$ & $0.9742 \pm 0.0114$ \\
had energy & $0.9901 \pm 0.0069$ & $0.9895 \pm 0.0074$ \\
$\Delta x_{\text {cmp }}$ & $0.9704 \pm 0.0119$ & $0.9692 \pm 0.0124$ \\
Track cut (\# SL) & $0.9951 \pm 0.0049$ & $0.9947 \pm 0.0052$ \\
\hline Total & $0.9310 \pm 0.0178$ & \\
\hline
\end{tabular}

Table A.6: The efficiency of the central muon (CMP only) identification cuts using the $Z^{0} \rightarrow \mu^{+} \mu^{-}$data sample. All uncertainties are statistical.

\begin{tabular}{c|cc}
\hline variable & individual & $\mathrm{N}-1$ \\
\hline \hline em energy & $0.9815 \pm 0.0065$ & $0.9806 \pm 0.0068$ \\
had energy & $0.9815 \pm 0.0065$ & $0.9806 \pm 0.0068$ \\
$\Delta x_{\text {cmx }}$ & $0.9792 \pm 0.0069$ & $0.9806 \pm 0.0068$ \\
Track cut (\# SL) & $0.9907 \pm 0.0046$ & $0.9926 \pm 0.0042$ \\
\hline Total & $0.9352 \pm 0.0118$ & \\
\hline
\end{tabular}

Table A.7: The efficiency of the central muon (CMX) identification cuts using the $Z^{0} \rightarrow \mu^{+} \mu^{-}$data sample. All uncertainties are statistical. 


\begin{tabular}{c|cc}
\hline variable & individual & $\mathrm{N}-1$ \\
\hline \hline em energy & $0.9734 \pm 0.0073$ & $0.9763 \pm 0.0083$ \\
had energy & $0.9857 \pm 0.0054$ & $0.9850 \pm 0.0066$ \\
em + had energy & $0.9570 \pm 0.0092$ & $0.9481 \pm 0.0119$ \\
Track cut (\# SL) & $0.7377 \pm 0.0199$ & $0.7360 \pm 0.0208$ \\
\hline Total & $0.6742 \pm 0.0212$ & \\
\hline
\end{tabular}

Table A.8: The efficiency of the minimum ionizing particle (CMIO) identification cuts using the $Z^{0} \rightarrow \mu^{+} \mu^{-}$data sample. All uncertainties are statistical.

\begin{tabular}{c|cc}
\hline variable & individual & $\mathrm{N}-1$ \\
\hline \hline$E_{\text {had }} / E_{\text {em }}$ & $0.9891 \pm 0.0003$ & $0.9892 \pm 0.0003$ \\
$\mathrm{E} / \mathrm{P}$ & $0.9353 \pm 0.0008$ & $0.9451 \pm 0.0008$ \\
$\mathrm{Lshr}$ & $0.9729 \pm 0.0005$ & $0.9754 \pm 0.0005$ \\
$Q \times \Delta x$ & $0.9858 \pm 0.0004$ & $0.9942 \pm 0.0003$ \\
$\Delta z$ & $0.9954 \pm 0.0002$ & $0.9991 \pm 0.0001$ \\
$\chi_{\text {strip }}^{2}$ & $0.9835 \pm 0.0004$ & $0.9859 \pm 0.0004$ \\
Track cut (\# SL) & $0.9923 \pm 0.0003$ & $0.9935 \pm 0.0003$ \\
\hline Total & $0.8759 \pm 0.0011$ & \\
\hline
\end{tabular}

Table A.9: The efficiency of the tight central electron (TCE) identification cuts using a $Z^{0} \rightarrow e^{+} e^{-}$PYTHIA sample. All uncertainties are statistical. 


\begin{tabular}{c|cc}
\hline variable & individual & $\mathrm{N}-1$ \\
\hline \hline$E_{\text {had }} / E_{\text {em }}$ & $0.9913 \pm 0.0003$ & $0.9917 \pm 0.0004$ \\
$\mathrm{PEM} \chi_{3 \times 3}^{2}$ & $0.9616 \pm 0.0007$ & $0.9673 \pm 0.0007$ \\
$\Delta R_{P E M-P E S}$ & $0.9878 \pm 0.0004$ & - \\
pesProfileRatio5by $9(\mathrm{U})$ & $0.9950 \pm 0.0003$ & $0.9966 \pm 0.0002$ \\
pesProfileRatio5by9 $9(\mathrm{~V})$ & $0.9960 \pm 0.0002$ & $0.9974 \pm 0.0002$ \\
\hline Total & $0.9405 \pm 0.0009$ & \\
\hline
\end{tabular}

Table A.10: The efficiency of the plug electron (PEM) identification cuts using a $Z^{0} \rightarrow e^{+} e^{-}$PYTHIA sample. All uncertainties are statistical.

\begin{tabular}{c|cc}
\hline variable & individual & $\mathrm{N}-1$ \\
\hline \hline$E_{\text {had }} / E_{\text {em }}$ & $0.9913 \pm 0.0003$ & $0.9921 \pm 0.0004$ \\
PEM $\chi_{3 \times 3}^{2}$ & $0.9616 \pm 0.0007$ & $0.9659 \pm 0.0008$ \\
$\Delta R_{P E M-P E S}$ & $0.9878 \pm 0.0004$ & - \\
pesProfileRatio5by $9(\mathrm{U})$ & $0.9950 \pm 0.0003$ & $0.9963 \pm 0.0003$ \\
pesProfileRatio5by $9(\mathrm{~V})$ & $0.9960 \pm 0.0002$ & $0.9974 \pm 0.0002$ \\
\hline Phoenix Track matching & $0.8081 \pm 0.0015$ & - \\
Phoenix Silicon hits & $0.9035 \pm 0.0012$ & $0.9032 \pm 0.0013$ \\
Phoenix Track $z_{0}$ & $0.9993 \pm 0.0001$ & $0.9994 \pm 0.0001$ \\
\hline Total & $0.6858 \pm 0.0018$ & \\
\hline
\end{tabular}

Table A.11: The efficiencies of the Phoenix electron (PHX) identification cuts using a $Z^{0} \rightarrow e^{+} e^{-}$PYTHIA sample. All uncertainties are statistical. The Phoenix Track matching cut is the efficiency that a PEM has a Phoenix track. The Phoenix Silicon hits, and Phoenix Track $z_{0}$ is obtained from the PEM that has a Phoenix track. The total efficiency is the probability that a PEM passes all PHX identification cuts and has a Phoenix track. 


\begin{tabular}{c|cc}
\hline variable & individual & $\mathrm{N}-1$ \\
\hline \hline em energy & $0.9589 \pm 0.0013$ & $0.9589 \pm 0.0013$ \\
had energy & $0.9787 \pm 0.0009$ & $0.9787 \pm 0.0009$ \\
$\Delta x_{c m u}$ & $0.9985 \pm 0.0002$ & $0.9994 \pm 0.0002$ \\
$\Delta x_{c m p}$ & $0.9941 \pm 0.0005$ & $0.9952 \pm 0.0005$ \\
Track cut (\# SL) & $0.9956 \pm 0.0004$ & $0.9958 \pm 0.0004$ \\
\hline Total & $0.9285 \pm 0.0016$ & \\
\hline
\end{tabular}

Table A.12: The efficiency of the central muon (CMUP) identification cuts using a $Z^{0} \rightarrow \mu^{+} \mu^{-}$PYTHIA sample. All uncertainties are statistical.

\begin{tabular}{c|cc}
\hline variable & individual & $\mathrm{N}-1$ \\
\hline \hline em energy & $0.9563 \pm 0.0028$ & $0.9565 \pm 0.0028$ \\
had energy & $0.9743 \pm 0.0021$ & $0.9744 \pm 0.0022$ \\
$\Delta x_{\text {cmu }}$ & $0.9991 \pm 0.0004$ & $0.9990 \pm 0.0004$ \\
Track cut (\# SL) & $0.9969 \pm 0.0007$ & $0.9969 \pm 0.0008$ \\
\hline Total & $0.9281 \pm 0.0035$ & \\
\hline
\end{tabular}

Table A.13: The efficiency of the central muon (CMU only) identification cuts using a $Z^{0} \rightarrow \mu^{+} \mu^{-}$PYTHIA sample. All uncertainties are statistical. 


\begin{tabular}{c|cc}
\hline variable & individual & $\mathrm{N}-1$ \\
\hline \hline em energy & $0.9774 \pm 0.0018$ & $0.9774 \pm 0.0019$ \\
had energy & $0.9837 \pm 0.0016$ & $0.9836 \pm 0.0016$ \\
$\Delta x_{\text {cmp }}$ & $0.9935 \pm 0.0010$ & $0.9939 \pm 0.0010$ \\
Track cut (\# SL) & $0.9952 \pm 0.0009$ & $0.9951 \pm 0.0009$ \\
\hline Total & $0.9507 \pm 0.0027$ & \\
\hline
\end{tabular}

Table A.14: The efficiency of the central muon (CMP only) identification cuts using a $Z^{0} \rightarrow \mu^{+} \mu^{-}$PYTHIA sample. All uncertainties are statistical.

\begin{tabular}{c|cc}
\hline variable & individual & $\mathrm{N}-1$ \\
\hline \hline em energy & $0.9585 \pm 0.0018$ & $0.9583 \pm 0.0019$ \\
had energy & $0.9681 \pm 0.0016$ & $0.9678 \pm 0.0017$ \\
$\Delta x_{\text {cmx }}$ & $0.9978 \pm 0.0004$ & $0.9979 \pm 0.0004$ \\
Track cut $(\#$ SL) & $0.9934 \pm 0.0007$ & $0.9934 \pm 0.0008$ \\
\hline Total & $0.9196 \pm 0.0025$ & \\
\hline
\end{tabular}

Table A.15: The efficiency of the central muon (CMX) identification cuts using a $Z^{0} \rightarrow \mu^{+} \mu^{-}$PYTHIA sample. All uncertainties are statistical. 


\begin{tabular}{c|cc}
\hline variable & individual & $\mathrm{N}-1$ \\
\hline \hline em energy & $0.9630 \pm 0.0015$ & $0.9609 \pm 0.0019$ \\
had energy & $0.9671 \pm 0.0015$ & $0.9661 \pm 0.0018$ \\
em + had energy & $0.9510 \pm 0.0018$ & $0.9351 \pm 0.0024$ \\
Track cut (\# SL) & $0.7786 \pm 0.0034$ & $0.7698 \pm 0.0037$ \\
\hline Total & $0.6792 \pm 0.0038$ & \\
\hline
\end{tabular}

Table A.16: The efficiency of the minimum ionizing particle (CMIO) identification cuts using a $Z^{0} \rightarrow \mu^{+} \mu^{-}$PYTHIA sample. All uncertainties are statistical.

\begin{tabular}{c|cc|c}
\hline Lepton type & Data & Monte Carlo (PYTHIA) & Scale factor \\
\hline \hline TCE & $0.8506 \pm 0.0058$ & $0.8759 \pm 0.0011$ & 0.971 \\
PEM & $0.8822 \pm 0.0069$ & $0.9405 \pm 0.0009$ & 0.938 \\
PHX & $0.4411 \pm 0.0107$ & $0.6858 \pm 0.0018$ & 0.643 \\
CMUP & $0.8561 \pm 0.0123$ & $0.9285 \pm 0.0016$ & 0.922 \\
CMU & $0.8906 \pm 0.0225$ & $0.9281 \pm 0.0035$ & 0.960 \\
CMP & $0.9310 \pm 0.0178$ & $0.9507 \pm 0.0027$ & 0.979 \\
CMX & $0.9352 \pm 0.0118$ & $0.9196 \pm 0.0025$ & 1.017 \\
CMIO & $0.6742 \pm 0.0212$ & $0.6792 \pm 0.0038$ & 0.993 \\
\hline
\end{tabular}

Table A.17: The ID efficiencies of leptons in data and a PYTHIA Monte Carlo sample. The scale factor is obtained from the efficiencies in the data divided by efficiencies in the Monte Carlo sample in each type of leptons. 

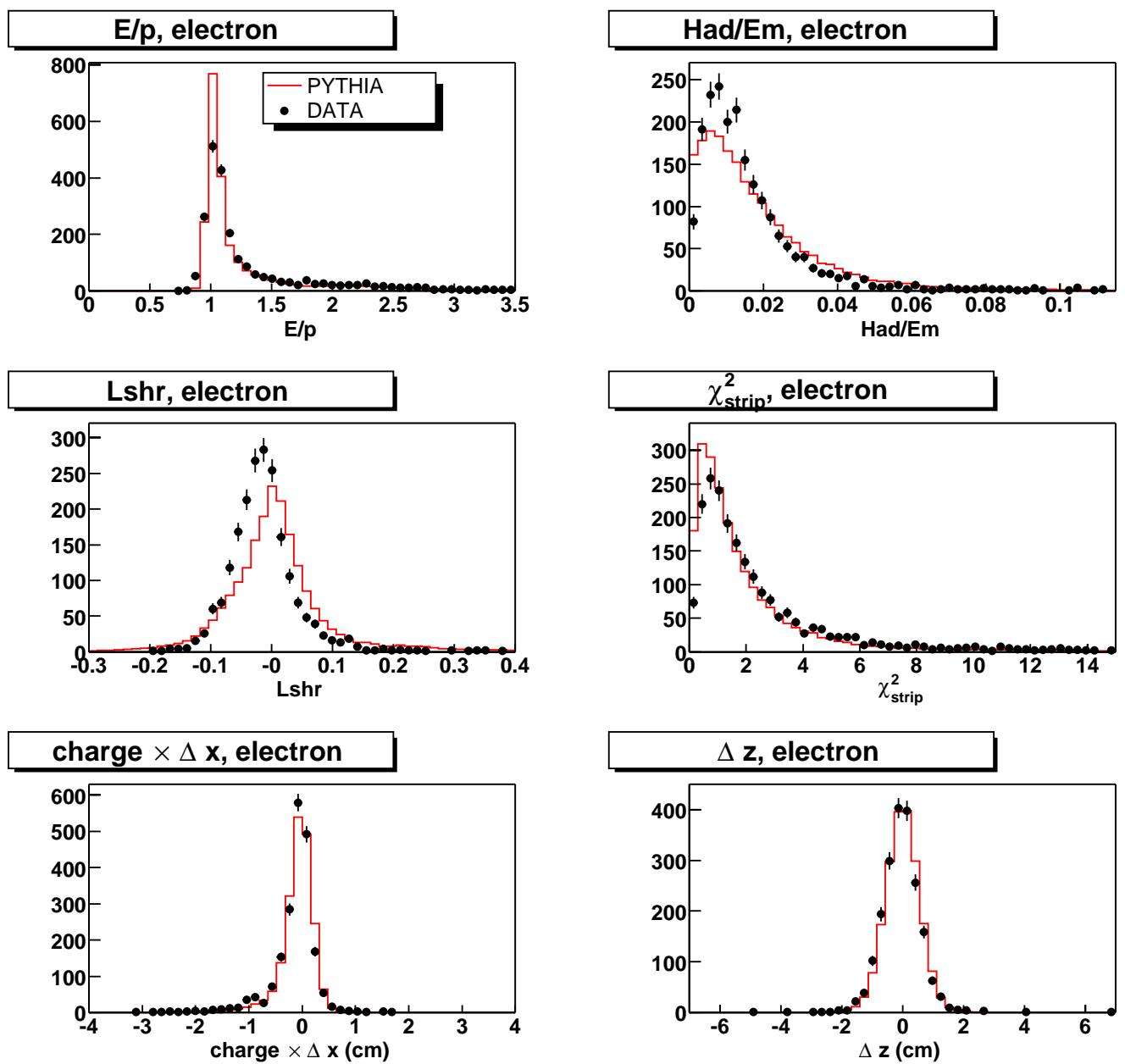

Figure A.1: The lepton identification variables of the central electron (TCE) obtained from the $Z^{0} \rightarrow e^{+} e^{-}$data sample (point) and a Monte Carlo sample (histogram). $E / p$ (top left), $E_{H A D} / E_{E M}$ (top right), Lshr (middle left), $\chi^{2}$ (middle right), charge $\times \Delta x$ (bottom left), $\Delta z$ (bottom right). 

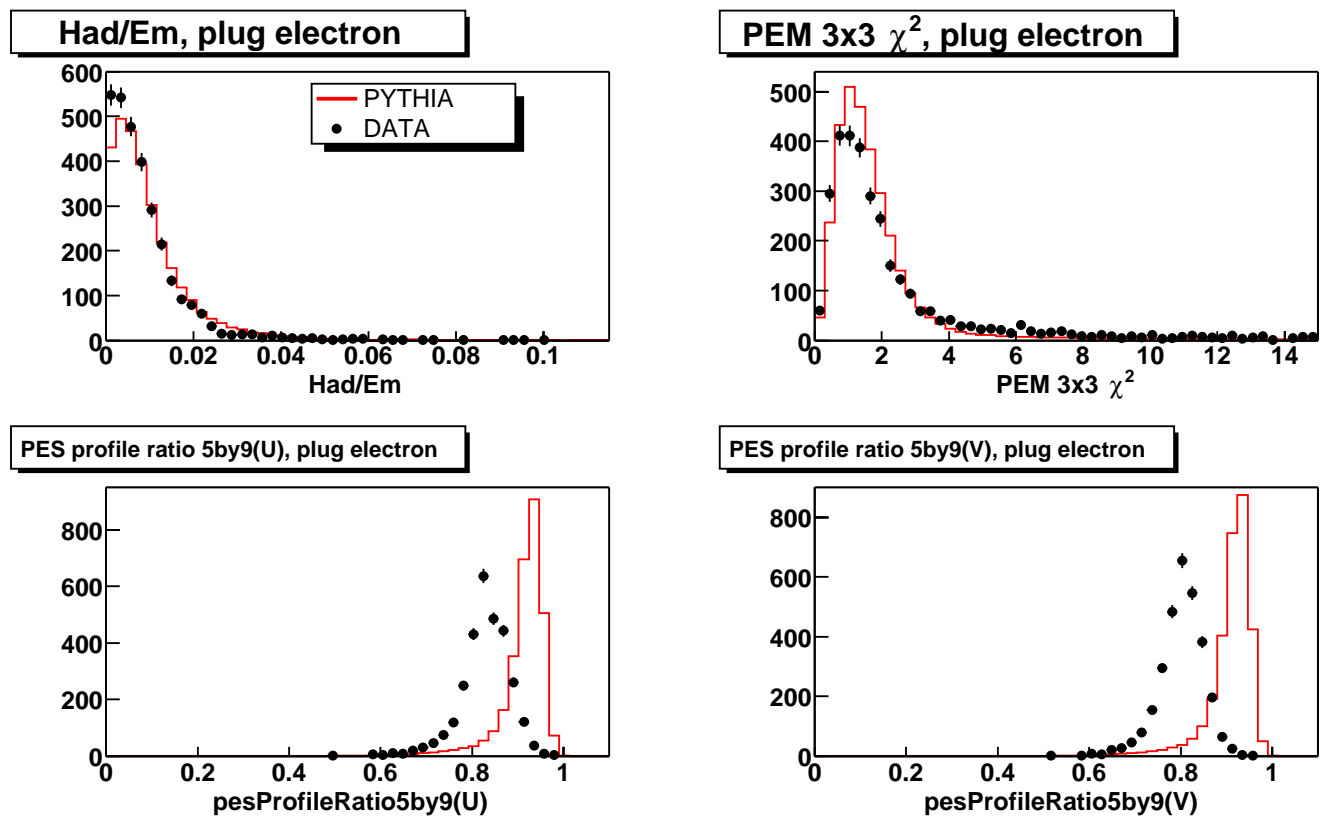

Figure A.2: The lepton identification variables of the plug electron (PEM) obtained from the $Z^{0} \rightarrow e^{+} e^{-}$data sample (point) and a Monte Carlo sample (histogram). $E_{H A D} / E_{E M}$ (top left), PEM $\chi_{3 \times 3}^{2}$ (top right), pesProfileRatio5by9(U) (bottom left), pesProfileRatio5by9(V) (bottom right). 

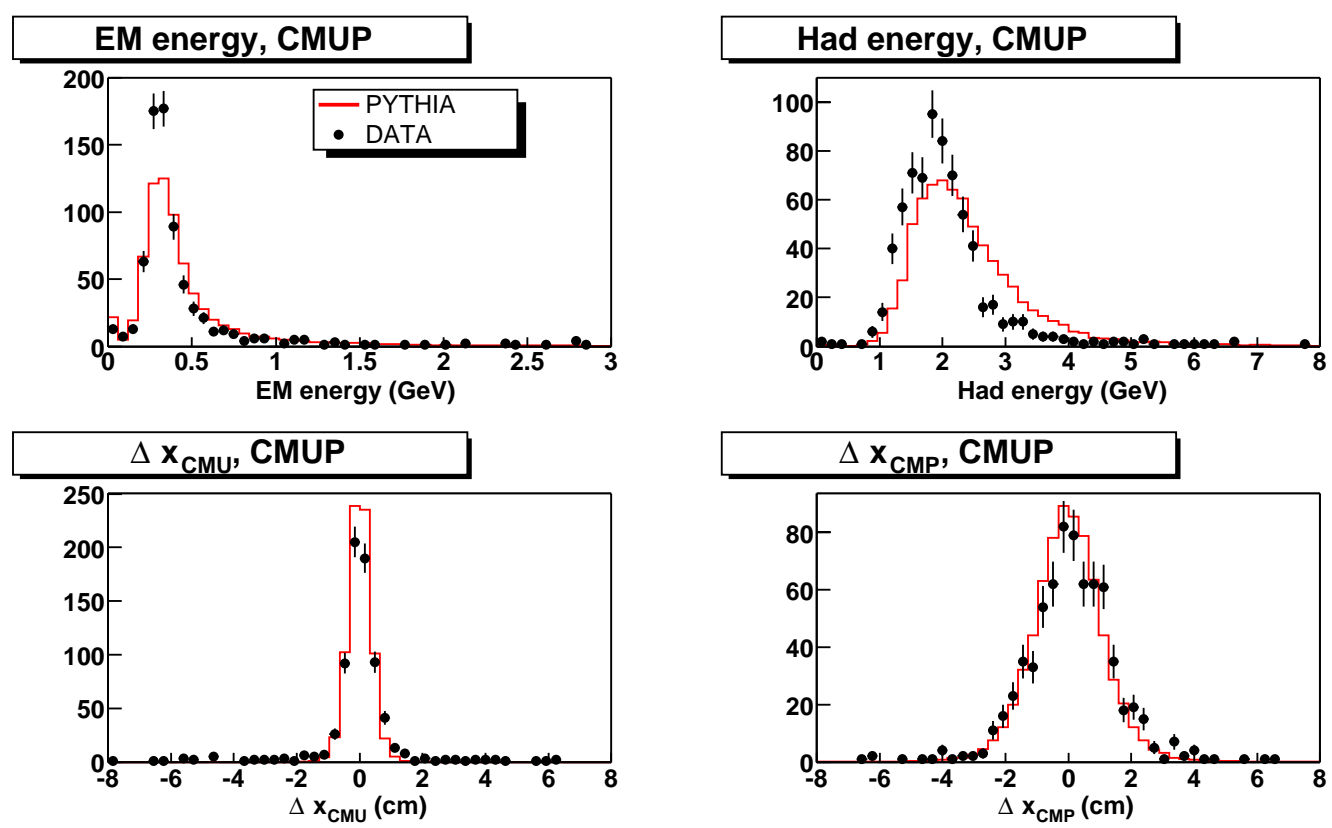

Figure A.3: The lepton identification variables of the central muon (CMUP) obtained from the $Z^{0} \rightarrow \mu^{+} \mu^{-}$data sample (point) and a Monte Carlo sample (histogram). $E_{E M}$ (top left), $E_{H A D}$ (top right), $\Delta x_{C M U}$ (bottom left), $\Delta x_{C M P}$ (bottom right). 

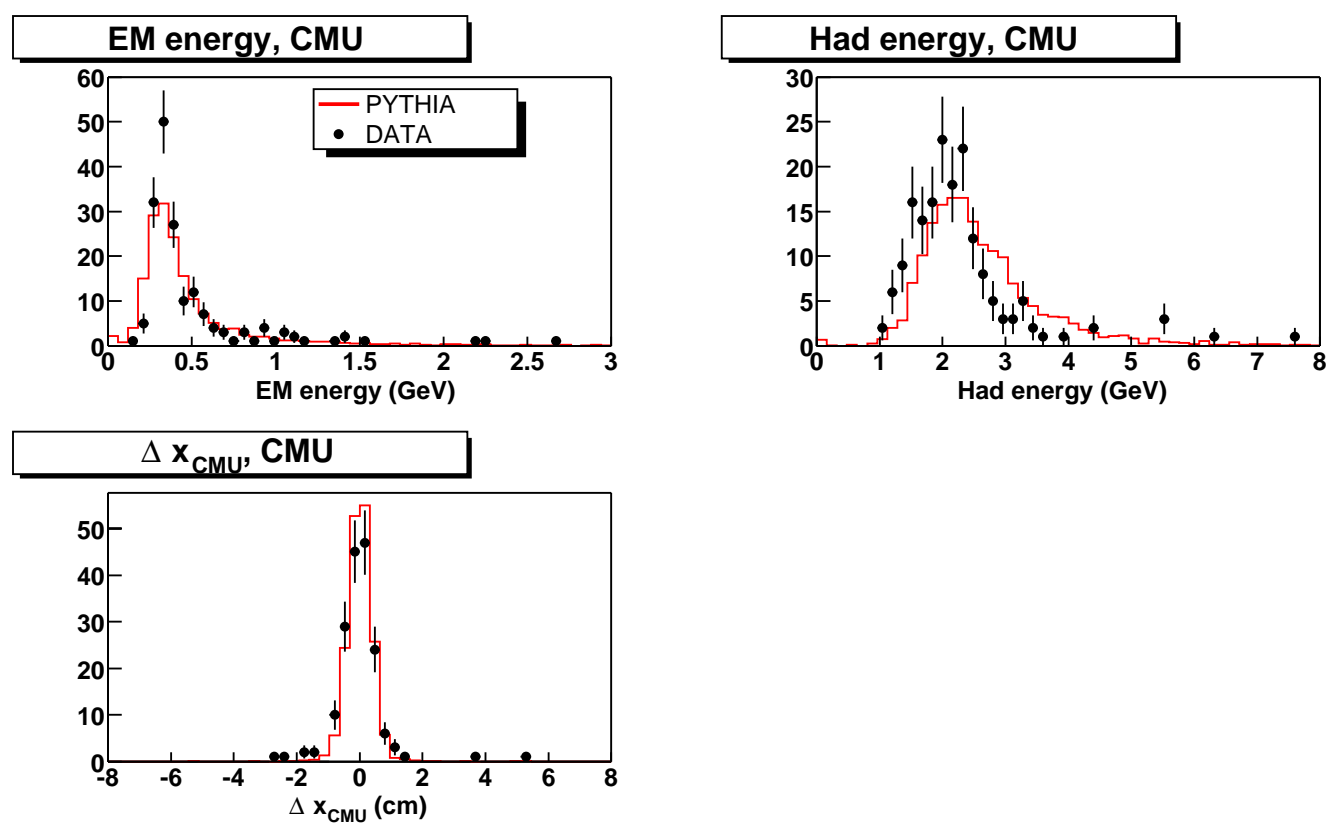

Figure A.4: The lepton identification variables of the central muon (CMU) obtained from the $Z^{0} \rightarrow \mu^{+} \mu^{-}$data sample (point) and a Monte Carlo sample (histogram). $E_{E M}$ (top left), $E_{H A D}$ (top right), $\Delta x_{C M U}$ (bottom left). 

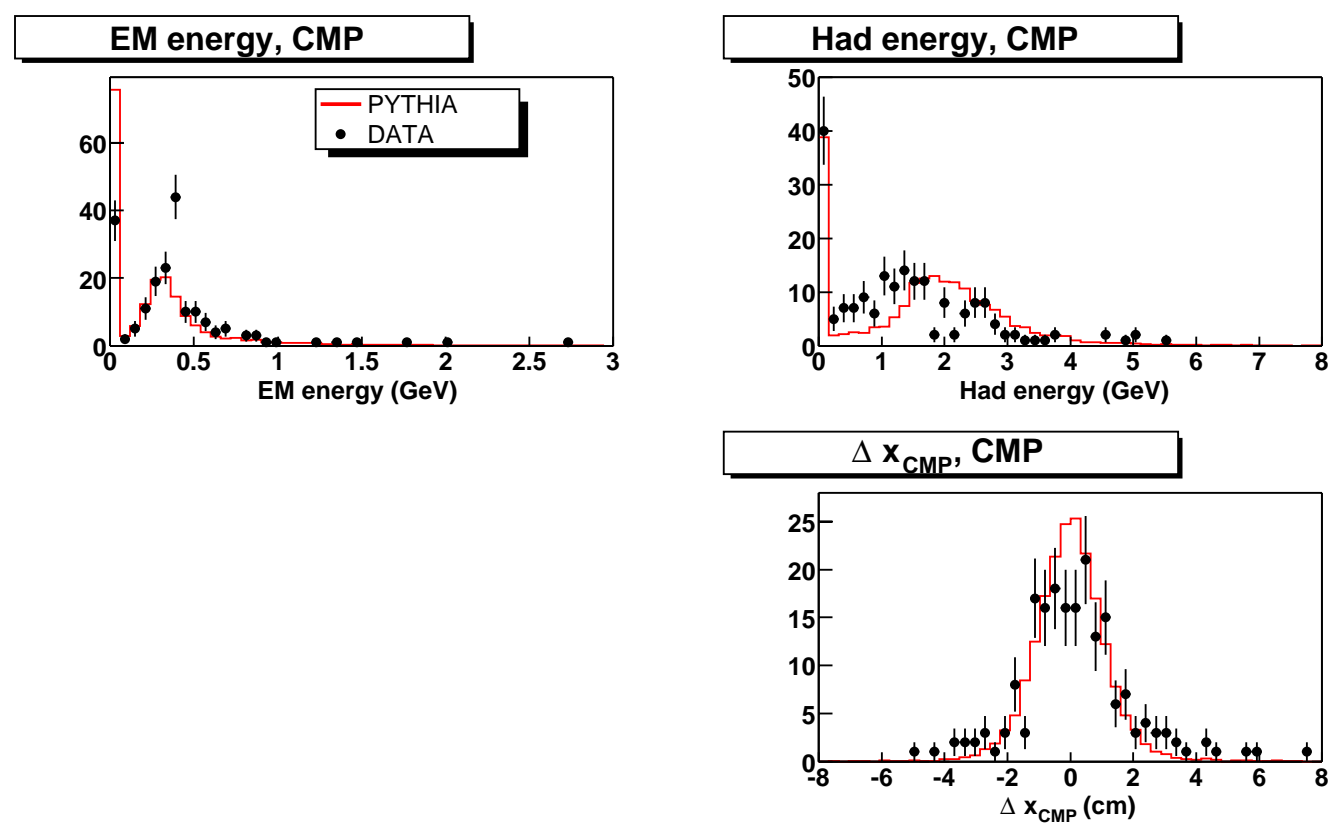

Figure A.5: The lepton identification variables of the central muon (CMP) obtained from the $Z^{0} \rightarrow \mu^{+} \mu^{-}$data sample (point) and a Monte Carlo sample (histogram). $E_{E M}$ (top left), $E_{H A D}$ (top right), $\Delta x_{C M P}$ (bottom right). 

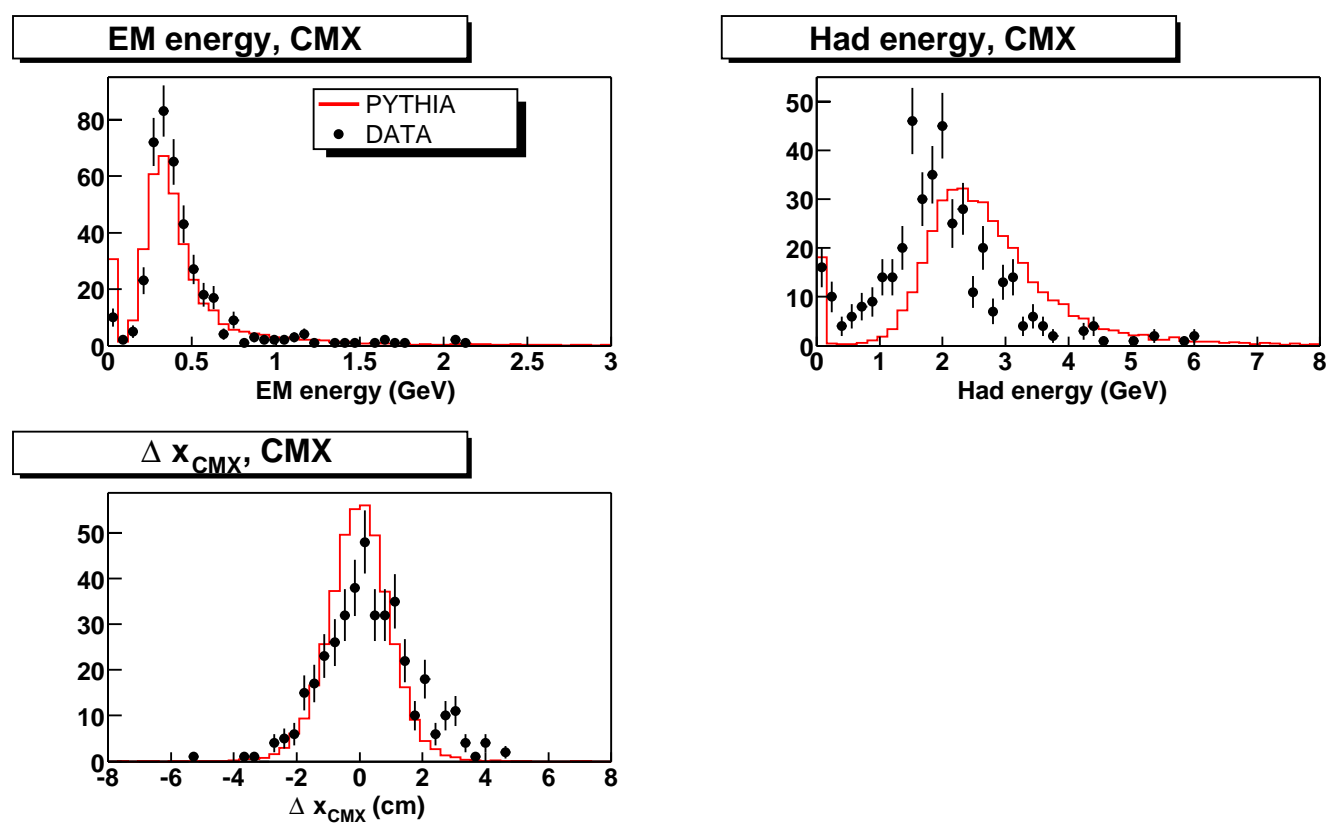

Figure A.6: The lepton identification variables of the central muon (CMX) obtained from the $Z^{0} \rightarrow \mu^{+} \mu^{-}$data sample (point) and a Monte Carlo sample (histogram). $E_{E M}$ (top left), $E_{H A D}$ (top right), $\Delta x_{C M X}$ (bottom left). 

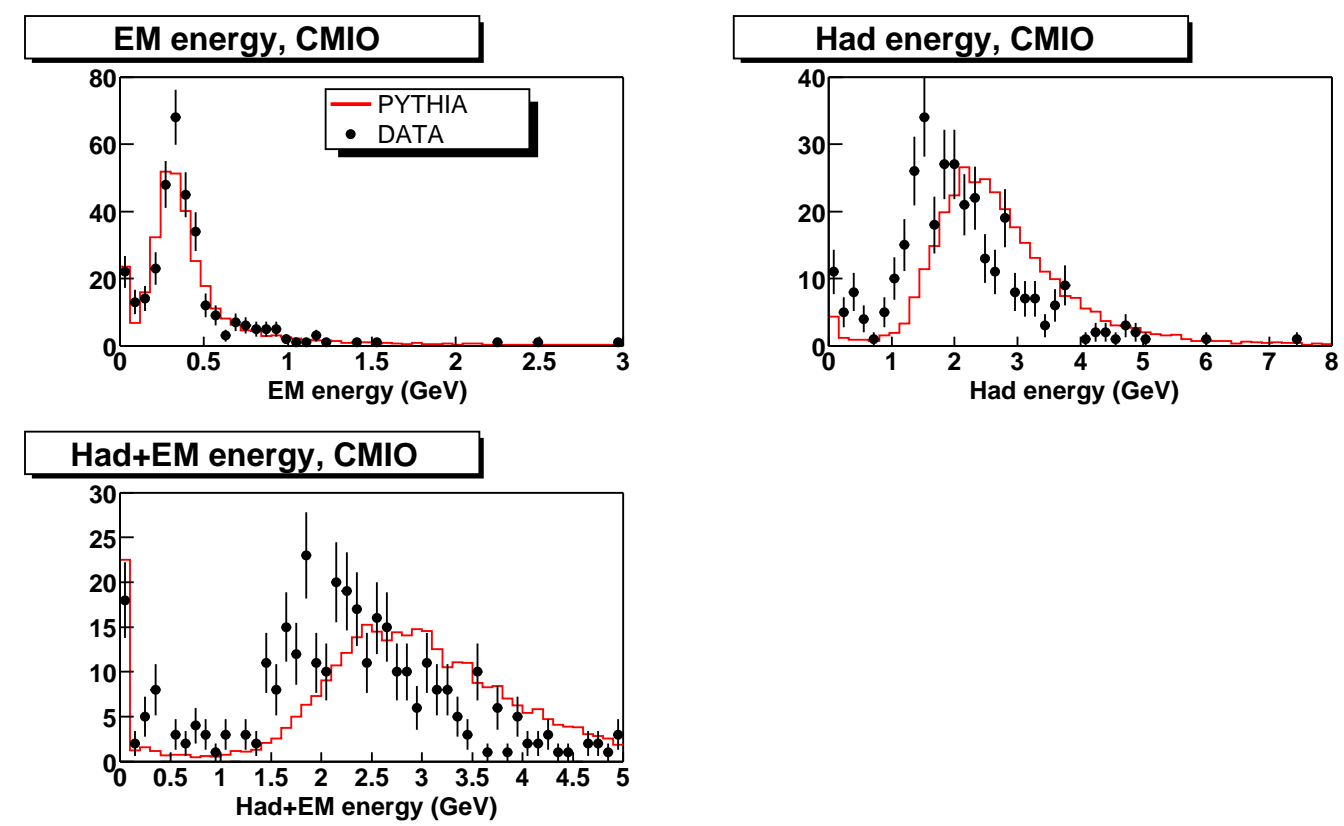

Figure A.7: The lepton identification variables of the minimum ionizing particle (CMIO) obtained from the $Z^{0} \rightarrow \mu^{+} \mu^{-}$data sample (point) and a Monte Carlo sample (histogram). $E_{E M}$ (top left), $E_{H A D}$ (top right), $E_{E M}+E_{H A D}$ (bottom left). 


\section{APPENDIX B}

\section{SELECTION OF THE OPTIMAL SCENARIO}

To investigate each scenario for dilepton selection, a technique based on pseudoexperiments is exploited. The cross section of the $t \bar{t}$ production is calculated as Equation B.1 where the $N_{o b s}$ is the number of the observed events passing the dilepton selections. The $N_{b g}^{e x p}$ is the expected number of background events for an integrated luminosity of $\mathcal{L}$. The $\epsilon_{d i l}$ is the dilepton channel acceptance.

$$
\sigma_{t \bar{t}}=\frac{N_{o b s}-N_{b g}^{e x p}}{\epsilon_{d i l} \mathcal{L}}
$$

For each pseudoexperiment, the number of observed events $\left(N_{o b s}\right)$ needs to be drawn randomly from a Poisson probability distribution function.

In order to obtain a random distribution of $N_{\text {obs }}$ for the pseudoexperiments, random distributions of $N_{t \bar{t}}$ and $N_{b g}$ are needed because $N_{o b s}$ can be obtained from $N_{t \bar{t}}+N_{b g}$. The $N_{t \bar{t}}$ and $N_{b g}$ are selected with the probability of $P_{\text {Poisson }}(x ; \mu)$ in Equation B.2,

$$
P_{\text {Poisson }}(x ; \mu)=\frac{\mu^{x} e^{-\mu}}{x !}
$$


where $x=N_{t \bar{t}}$ and $x=N_{b g}$ with the Poisson mean $\mu=N_{t \bar{t}}^{e x p}$ and $\mu=N_{b g}^{e x p}$ respectively. The $N_{t \bar{t}}^{e x p}$ and $N_{b g}^{e x p}$ are the $t \bar{t}$ events and the background events expected for each dilepton selection scenario and an integrated luminosity $\mathcal{L}$.

The procedure is repeated for $N_{p e}$ pseudoexperiments to determine a distribution of possible cross section measurements. The width obtained from the cross section distribution corresponds to the a priori statistical uncertainty of the cross section measurement. The procedure of extracting a priori statistical uncertainty of the cross section will be denoted as Step 1. To incorporate the systematic effect from the $t \bar{t}$ acceptance and the background estimates, the expected number of $t \bar{t}$ events and background events are smeared with the Gaussian probability distribution function (this will be denoted as Step 2). The Gaussian smeared $\hat{N}_{t \bar{t}}$ is obtained with the probability given by Equation B.3 with $x=\hat{N}_{t \bar{t}}, \mu=N_{t \bar{t}}^{e x p}$, and $\sigma=\delta N_{t \bar{t}}^{e x p}$ which is the error of $N_{t \bar{t}}^{e x p}$. The $\hat{N}_{b g}$ is smeared in the similar way. Then, $N_{t \bar{t}}$ and $N_{b g}$ are randomized with the probability of $P_{\text {Poisson }}(x ; \mu)$ from the Equation B.2 where $x=N_{t \bar{t}}$ and $x=N_{b g}$ with the Poisson means $\mu=\hat{N}_{t \bar{t}}$ and $\mu=\hat{N}_{b g}$ respectively. The cross section distribution obtained from $N_{o b s}=N_{t \bar{t}}+N_{b g}$ smeared in this way, includes the proper systematic effects.

$$
P_{\text {Gaussian }}(x ; \mu, \sigma)=\frac{1}{\sigma \sqrt{2 \pi}} e^{-(x-\mu)^{2} / 2 \sigma^{2}}
$$

With these procedures, the pseudoexperiment technique was applied to three different scenarios for the dilepton selections. The first one uses all 26 dilepton categories and the dilepton selection requirements as described in Section 3.3. The second one used the same selection requirements but does not use the dilepton categories involving the plug electron (PHX, PEM) and CMIO. The option reduces the 
$t \bar{t}$ acceptance but benefits from a smaller uncertainty of the background estimation. In the last scenario, all 26 dilepton categories are used while the isolation requirement allows one lepton to be non-isolated. Also it removes the $Z^{0}$ invariant mass cut. The cross section measurement using the last scenario has been performed by CDF Run II Dilepton Working Group in summer 2003. The details of the analysis are found in [36] [37]. These scenarios are summarized in Table B.1.

From the three scenarios, the expected numbers of $t \bar{t}$ events and the background estimations at various luminosities are obtained via the methods described in Chapters 3 and 4 . For the estimation of the a priori uncertainties, three integrated luminosities $\left(200 p b^{-1}, 500 p b^{-1}\right.$, and $\left.2 f b^{-1}\right)$ are considered. The expected numbers of $t \bar{t}$ and background events at these luminosities are listed in Table B.2 with their respective errors. For each of the three luminosities, 100,000 pseudoexperiments have been performed. The results of the pseudoexperiments are shown in Figures B.1, B.2, and B.3 for the luminosities of $200 \mathrm{pb}^{-1}, 500 \mathrm{pb} b^{-1}$, and $2 \mathrm{fb}^{-1}$. The RMS values of the distributions represent the a priori uncertainties of the cross section measurements. The RMS values are listed in Tables B.3, B.4, and B.5 for Step 1 and Step 2 pseudoexperiments. As discussed before, the uncertainty of Step 1 represents the statistical error only while the uncertainty of Step 2 has the combined effect from the statistical and systematic uncertainties. It is also worthwhile to consider only the systematic effect, which can be extracted from the uncertainties of Step 1 and 2 by

$$
\delta \sigma_{t \bar{t}}^{s y s t}=\sqrt{\delta \sigma_{t \bar{t}, S t e p 2}^{2}-\delta \sigma_{t \bar{t}, S t e p 1}^{2}} .
$$


For all three luminosities, the systematic uncertainty of each scenario does not vary much. In this a priori study, the systematic uncertainty is assumed not to scale down with luminosity. As expected, Scenario 2 gives the smallest systematic uncertainty due to its small backgrounds relative to the $t \bar{t}$ signal. Scenario 2 has the largest statistical uncertainty in $200 \mathrm{pb}^{-1}$ but the differences in the statistical uncertainties of the scenarios decreases as the luminosity increases because the statistical uncertainty is inversely proportional to the square root of the luminosity. Therefore, the total uncertainty of Scenario 2 becomes smaller than the uncertainties of other scenarios at higher luminosities though at $200 p b^{-1}$ it is the largest.

Currently, the available integrated luminosity is under $200 p b^{-1}$. However, by the end of 2004, the integrated luminosity of more than $500 p b^{-1}$ is expected. Scenario 2 will allow more sensitivity in the higher luminosity era. Given this consideration, Scenario 2 is used for the dilepton selection in this thesis. 


\begin{tabular}{c|c}
\hline & Dilepton Selection \\
\hline \hline Scenario 1 & $\begin{array}{r}\text { 26 dilepton categories including PHX, PEM, CMIO. } \\
\text { Dilepton selections described in Section 3.3. }\end{array}$ \\
\hline Scenario 2 & $\begin{array}{r}12 \text { dilepton categories without PHX, PEM, CMIO. } \\
\text { Dilepton selections described in Section 3.3. }\end{array}$ \\
\hline Scenario 3 & $\begin{array}{c}\text { One lepton in the event is allowed to be non-isolated. } \\
Z^{0} \text { invariant mass cut removed }\end{array}$ \\
& and replaced with alternative cuts [36][37]. \\
\hline
\end{tabular}

Table B.1: Three scenarios of dilepton selections for the study of a priori cross section uncertainty. 


\begin{tabular}{l|c|ccc}
\hline & & $200 p b^{-1}$ & $500 p b^{-1}$ & $2 \mathrm{fb}^{-1}$ \\
\hline \hline & $N_{t \bar{t}}^{e x p}$ & $8.62 \pm 0.83$ & $21.56 \pm 2.06$ & $86.24 \pm 8.26$ \\
Scenario 1 & $N_{b g}^{e x p}$ & $2.79 \pm 1.01$ & $6.97 \pm 2.52$ & $27.89 \pm 10.09$ \\
& $\epsilon_{\text {dil }} \mathcal{L}$ & $1.28 p b^{-1}$ & $3.2 p b^{-1}$ & $12.8 p b^{-1}$ \\
\hline \multirow{3}{*}{ Scenario 2 } & $N_{t \bar{t}}^{\text {exp }}$ & $6.94 \pm 0.83$ & $17.36 \pm 2.08$ & $69.44 \pm 8.33$ \\
& $N_{b g}^{\text {exp }}$ & $0.83 \pm 0.33$ & $2.08 \pm 0.83$ & $8.33 \pm 3.33$ \\
& $\epsilon_{\text {dil }} \mathcal{L}$ & $1.02 p b^{-1}$ & $2.58 p b^{-1}$ & $10.38 p b^{-1}$ \\
\hline \multirow{3}{*}{ Scenario 3 } & $N_{t \bar{t}}^{\text {exp }}$ & $10.0 \pm 1.11$ & $25.0 \pm 2.78$ & $100.0 \pm 11.1$ \\
& $N_{b g}^{\text {exp }}$ & $4.60 \pm 1.43$ & $11.51 \pm 3.57$ & $46.03 \pm 14.29$ \\
& $\epsilon_{\text {dil }} \mathcal{L}$ & $1.48 p b^{-1}$ & $3.71 p b^{-1}$ & $14.84 p b^{-1}$ \\
\hline
\end{tabular}

Table B.2: Three scenarios of dilepton selections for the study of a priori cross section uncertainty.

\begin{tabular}{c|ccc}
\hline \multirow{2}{*}{$200 p b^{-1}$} & \multicolumn{3}{|c}{$\operatorname{RMS}\left(\delta \sigma_{\bar{t} \bar{t}}^{p e}, p b\right)$} \\
\cline { 2 - 4 } & Step 1 $\left(\delta \sigma_{t \bar{t}, \text { Step } 1}\right)$ & Step 2 $\left(\delta \sigma_{t \bar{t}, \text { Step } 2}\right)$ & $\delta \sigma_{t \bar{t}}^{\text {syst }}$ \\
\hline \hline Scenario 1 & 2.64 & 2.82 & 0.98 \\
Scenario 2 & 2.73 & 2.86 & 0.83 \\
Scenario 3 & 2.58 & 2.84 & 1.20 \\
\hline
\end{tabular}

Table B.3: The result of the pseudoexperiments (100,000 events) in $200 p b^{-1}$. The RMS values of the cross section distribution of the pseudoexperiments $\left(\delta \sigma_{t \bar{t}}^{p e}\right)$ represent the a priori uncertainties of the cross section measurements. 


\begin{tabular}{c|ccc}
\hline & \multicolumn{3}{|c}{ RMS $\left(\delta \sigma_{t \bar{t}}^{p e}, p b\right)$} \\
\cline { 2 - 4 } $500 p b^{-1}$ & Step 1 $\left(\delta \sigma_{t \bar{t}, \text { Step } 1}\right)$ & Step 2 $\left(\delta \sigma_{t \bar{t}, \text { Step } 2}\right)$ & $\delta \sigma_{t \bar{t}}^{\text {syst }}$ \\
\hline \hline Scenario 1 & 1.67 & 1.96 & 1.02 \\
Scenario 2 & 1.72 & 1.92 & 0.86 \\
Scenario 3 & 1.63 & 2.03 & 1.21 \\
\hline
\end{tabular}

Table B.4: The result of the pseudoexperiments $\left(100,000\right.$ events) in $500 p b^{-1}$. The RMS values of the cross section distribution of the pseudoexperiments $\left(\delta \sigma_{t \bar{t}}^{p e}\right)$ represent the a priori uncertainties of the cross section measurements.

\begin{tabular}{c|ccc}
\hline & \multicolumn{3}{|c}{ RMS $\left(\delta \sigma_{t \bar{t}}^{p e}, p b\right)$} \\
\cline { 2 - 4 } $2 f b^{-1}$ & Step 1 $\left(\delta \sigma_{t \bar{t}, \text { Step } 1}\right)$ & Step 2 $\left(\delta \sigma_{t \bar{t}, \text { Step } 2}\right)$ & $\delta \sigma_{t \bar{t}}^{\text {syst }}$ \\
\hline \hline Scenario 1 & 0.84 & 1.32 & 1.02 \\
Scenario 2 & 0.85 & 1.21 & 0.86 \\
Scenario 3 & 0.82 & 1.46 & 1.21 \\
\hline
\end{tabular}

Table B.5: The result of the pseudoexperiments (100,000 events) in $2 f b^{-1}$. The RMS values of the cross section distribution of the pseudoexperiments $\left(\delta \sigma_{t \bar{t}}^{p e}\right)$ represent the a priori uncertainties of the cross section measurements. 

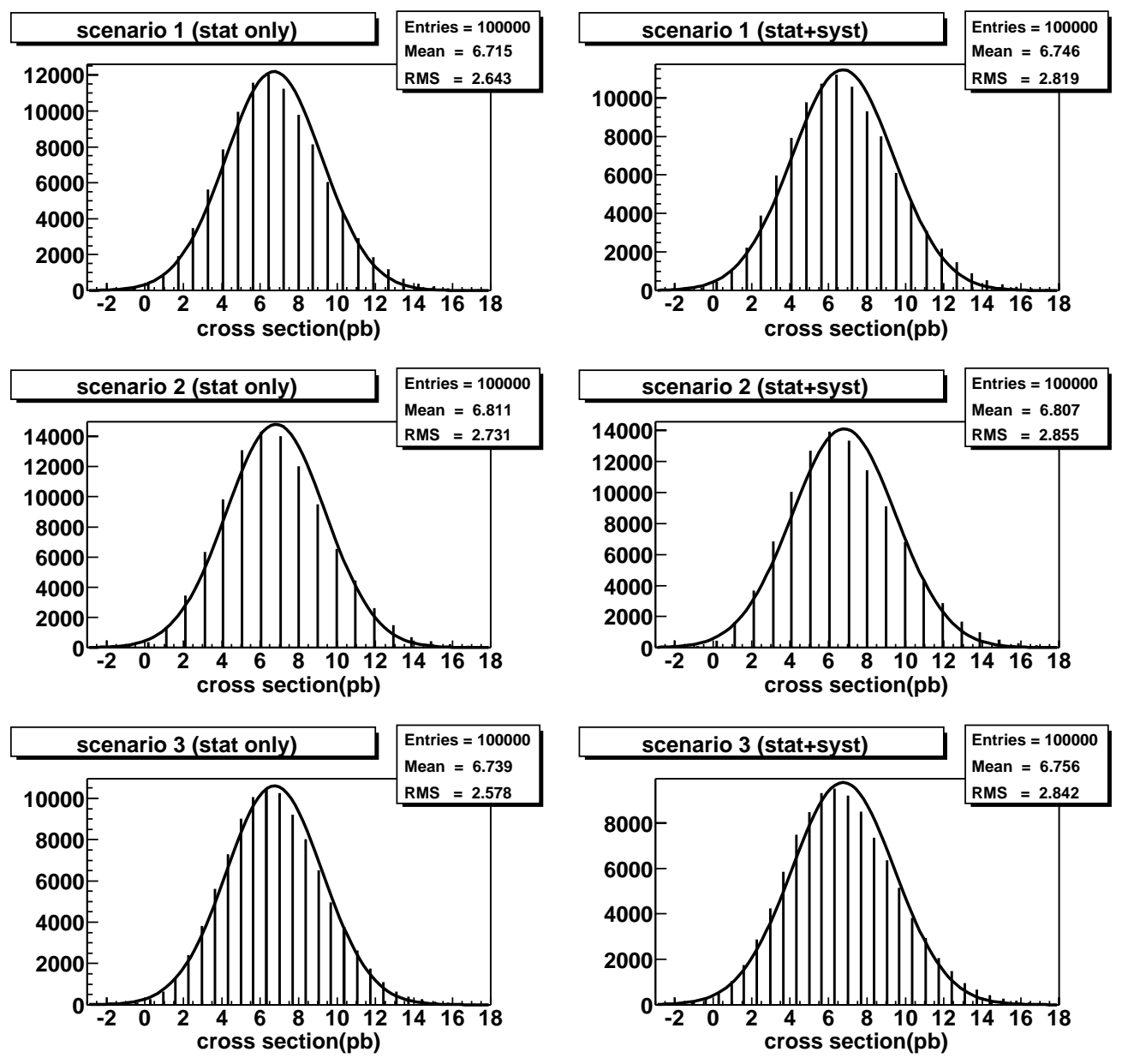

Figure B.1: The result of the pseudoexperiments (100,000 events) in $200 p b^{-1}$. The plots on the left are obtained from the step 1 procedure in the text. The plots on the right are obtained from the step 2 procedure in the text. The distributions of the cross sections are shown with a Gaussian fit. 

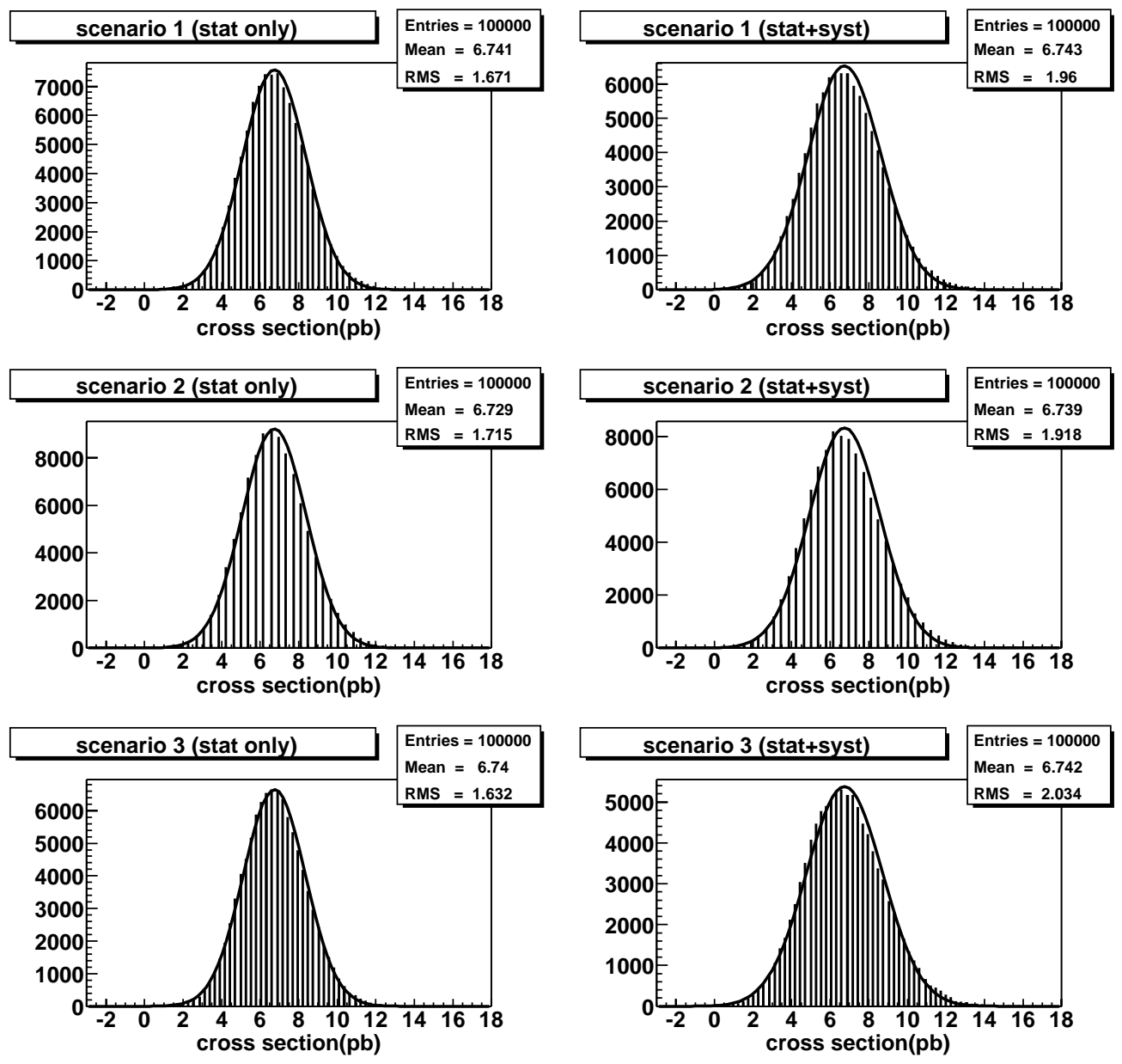

Figure B.2: The result of the pseudoexperiments (100,000 events) in $500 p b^{-1}$. The plots on the left are obtained from the step 1 procedure in the text. The plots on the right are obtained from the step 2 procedure in the text. The distributions of the cross sections are shown with a Gaussian fit. 

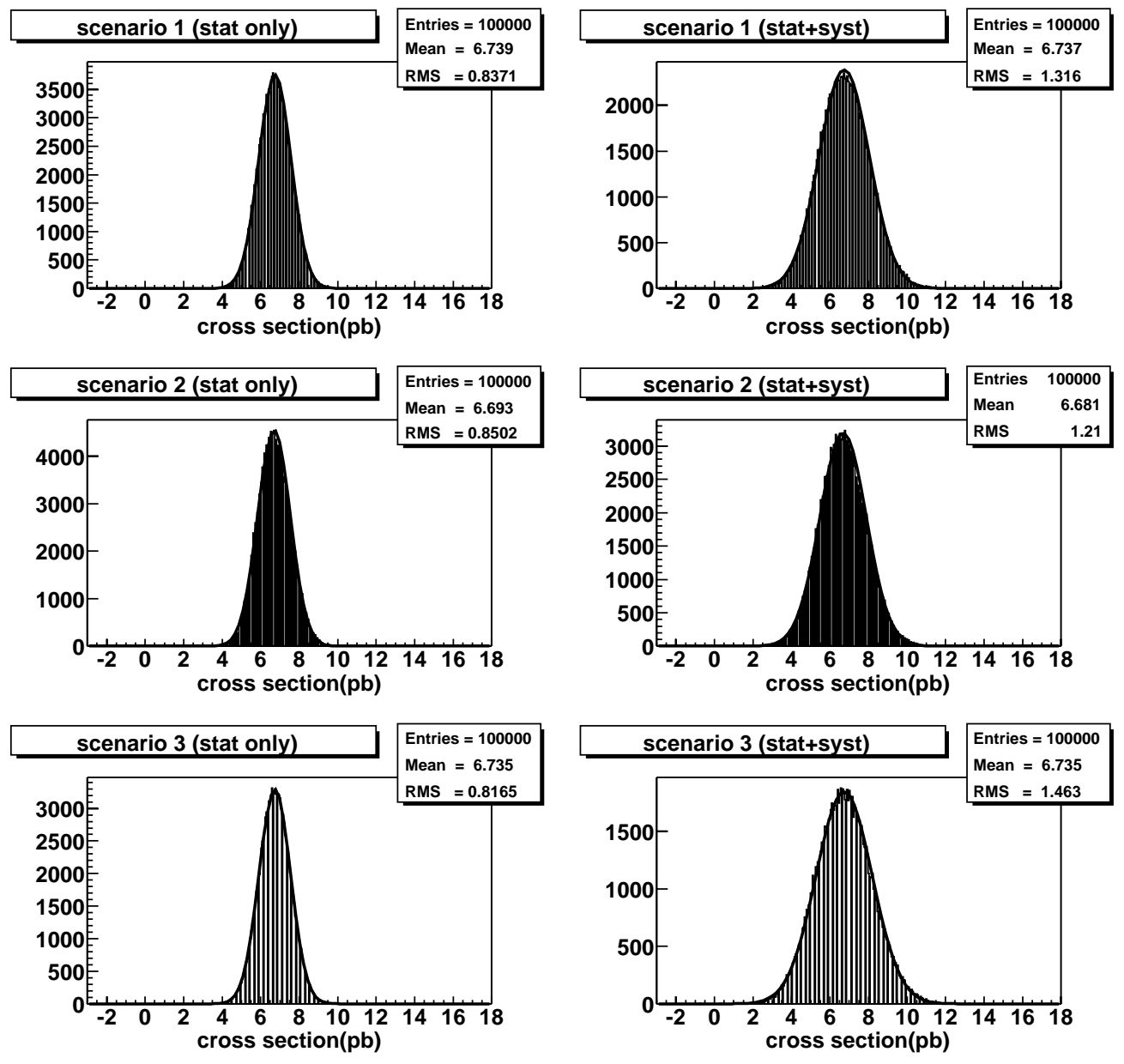

Figure B.3: The result of the pseudoexperiments (100,000 events) in $2 \mathrm{fb}^{-1}$. The plots on the left are obtained from the step 1 procedure in the text. The plots on the right are obtained from the step 2 procedure in the text. The distributions of the cross sections are shown with a Gaussian fit. 


\section{APPENDIX C}

\section{EVENT DISPLAYS OF THE DILEPTON CANDIDATES}

The event displays of the dilepton candidates described in Section 5.2 are shown

in this appendix. Each event has two event views. The first is a transverse view of the tracks in the events. The second is a view of the transverse energy deposited in the calorimeter towers in the $\eta-\phi$ plane. The quantities used in these event displays are the raw values before corrections. They may be different from the quantities used for the dilepton selections in Table 5.4. 


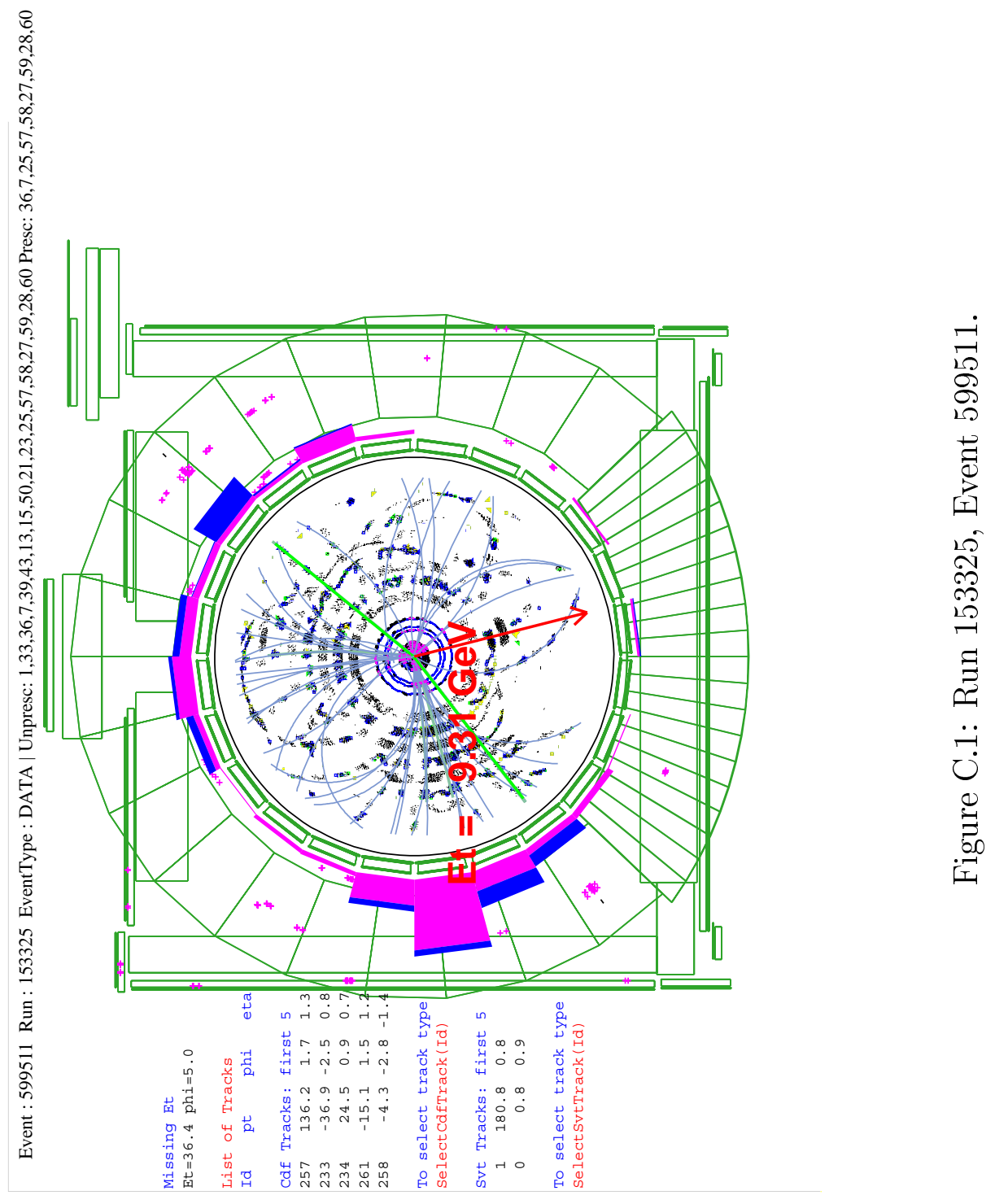




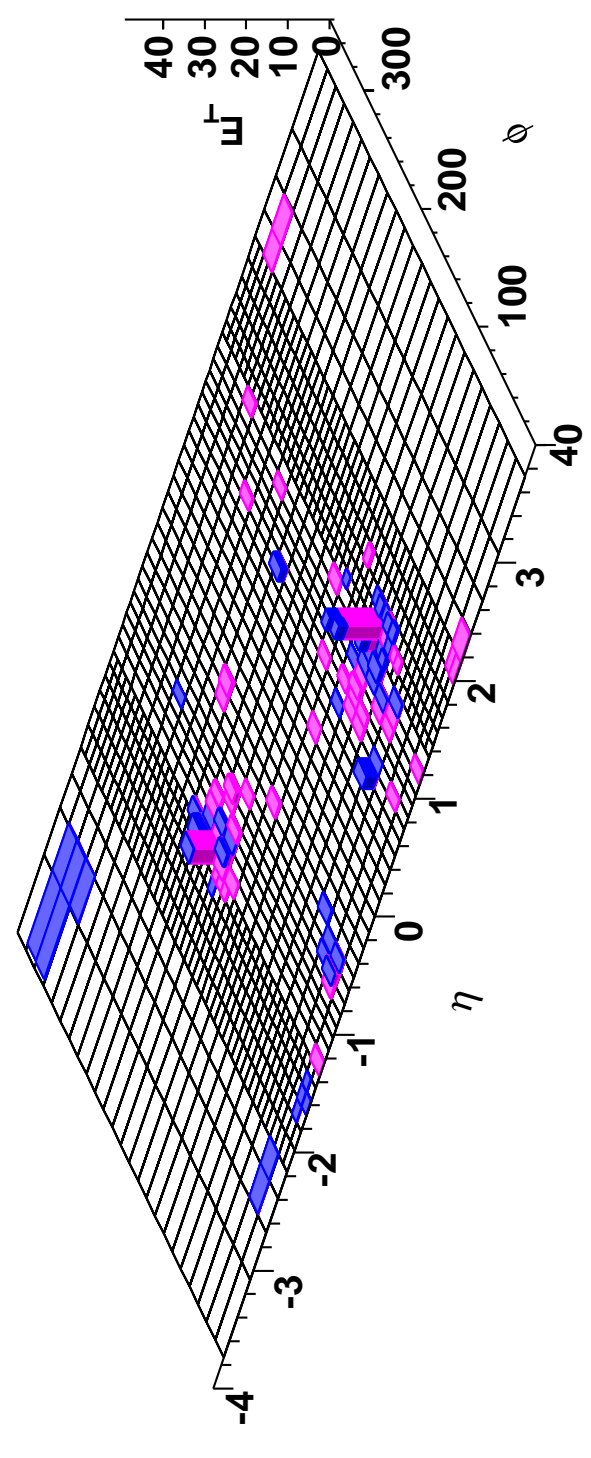

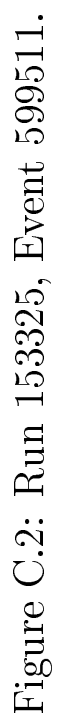




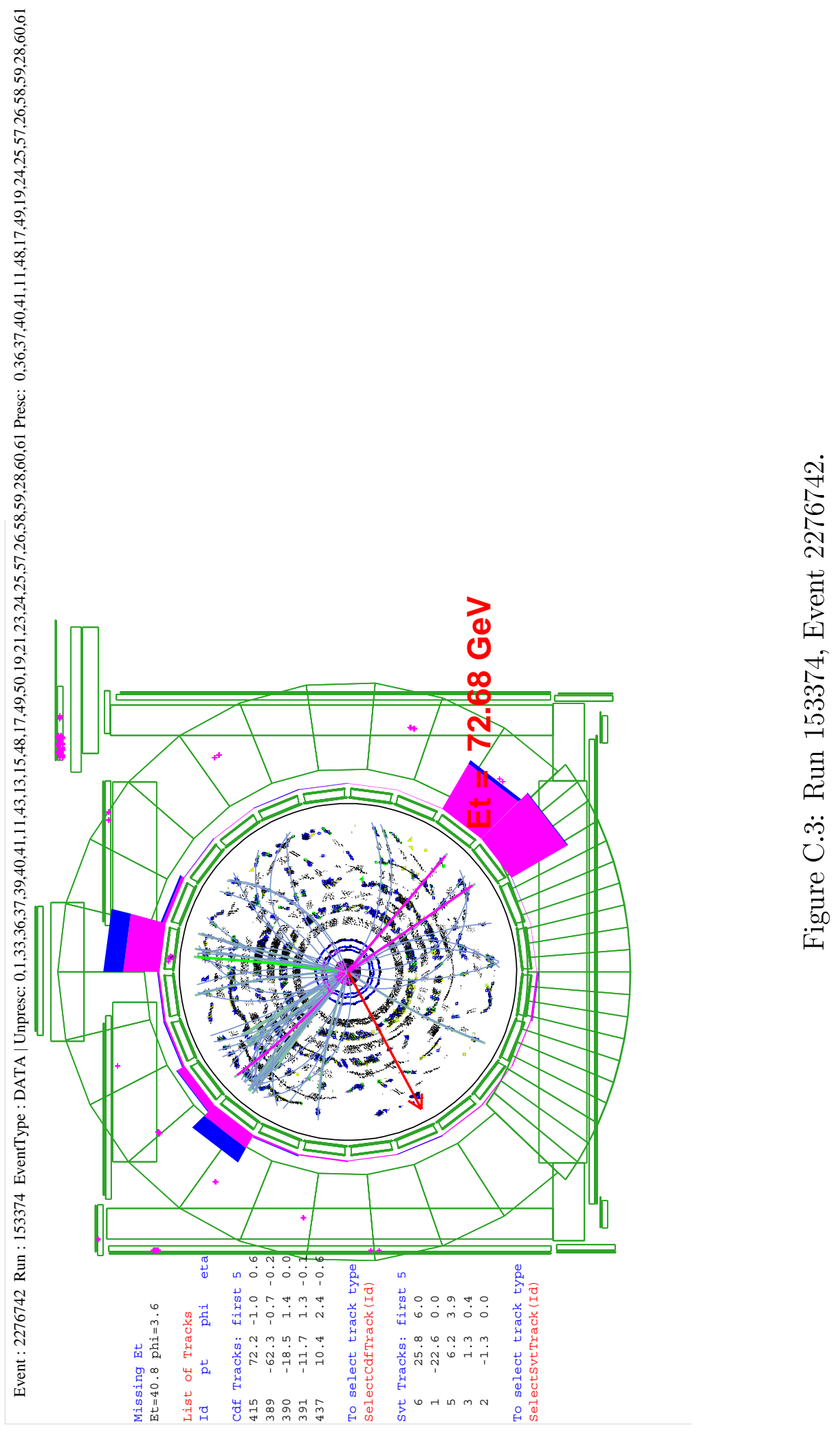




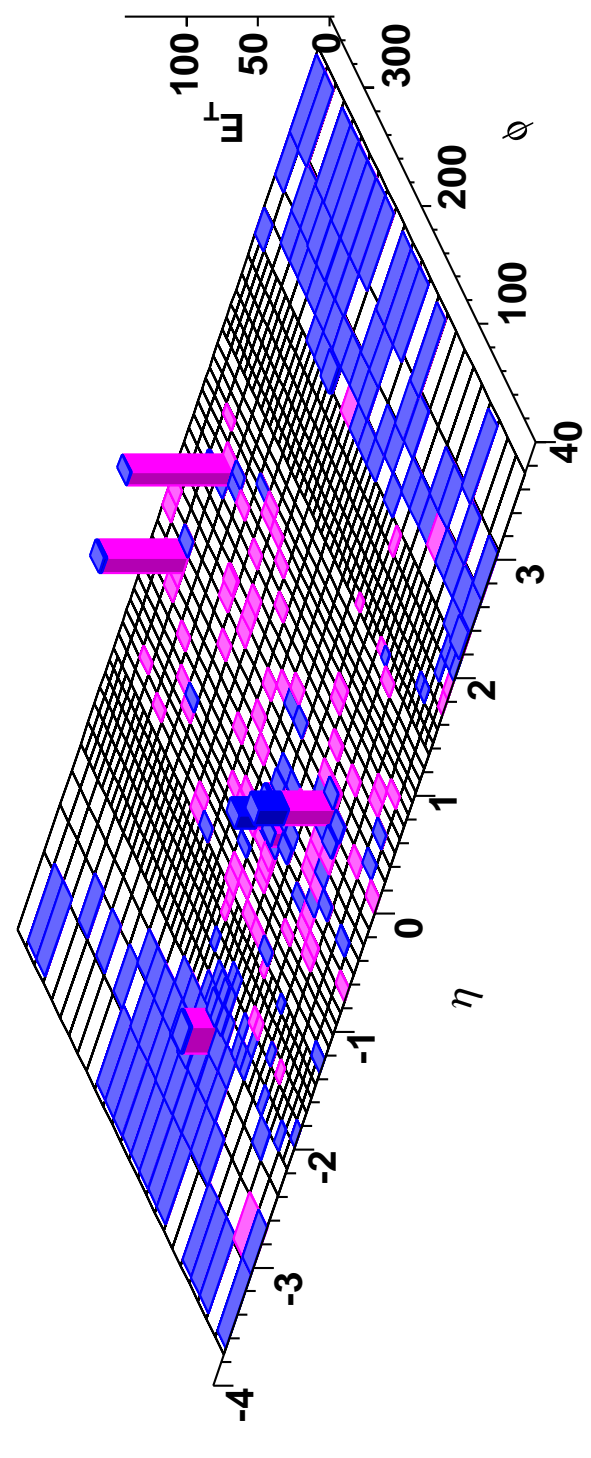

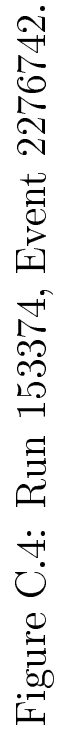




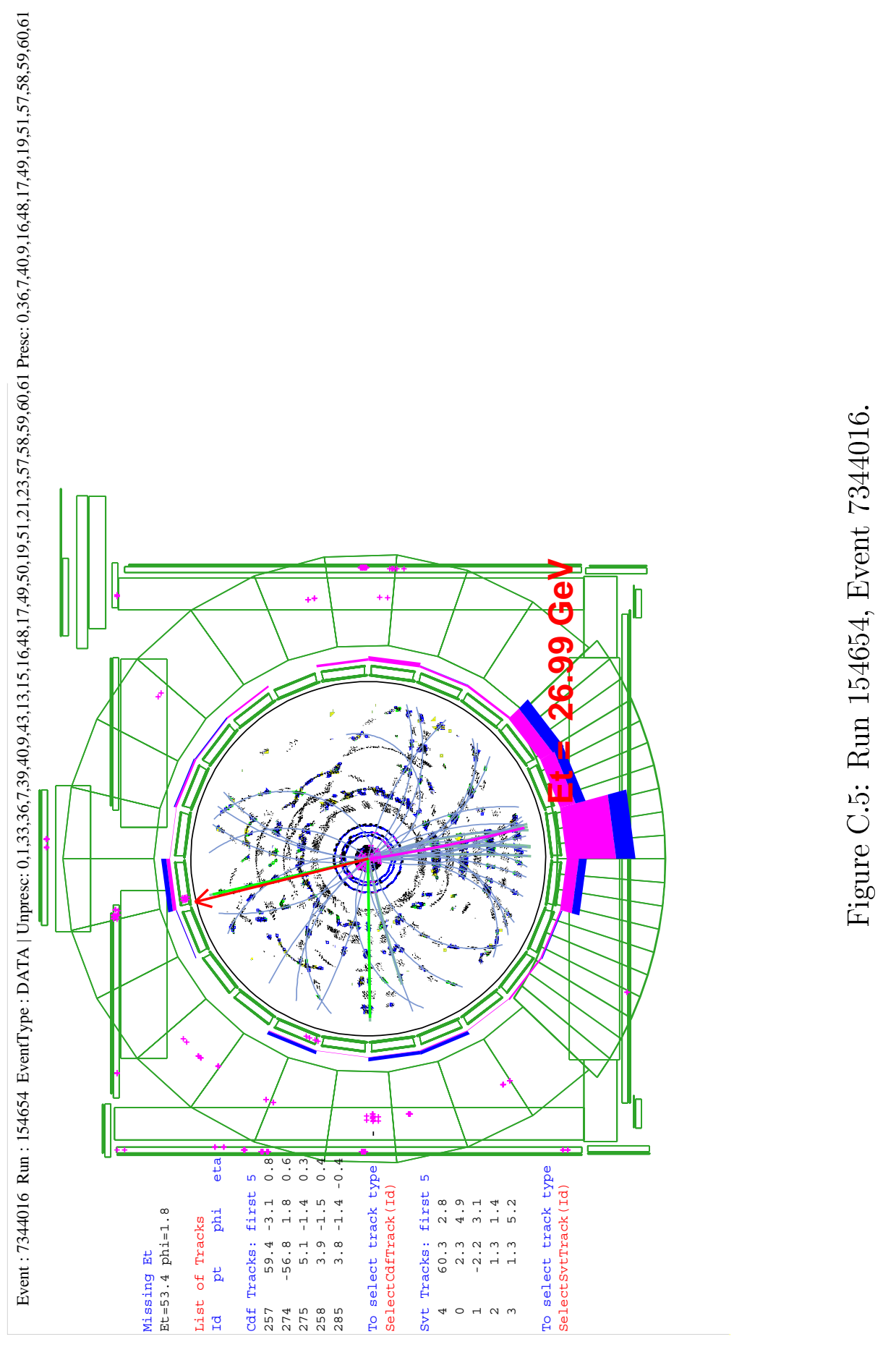




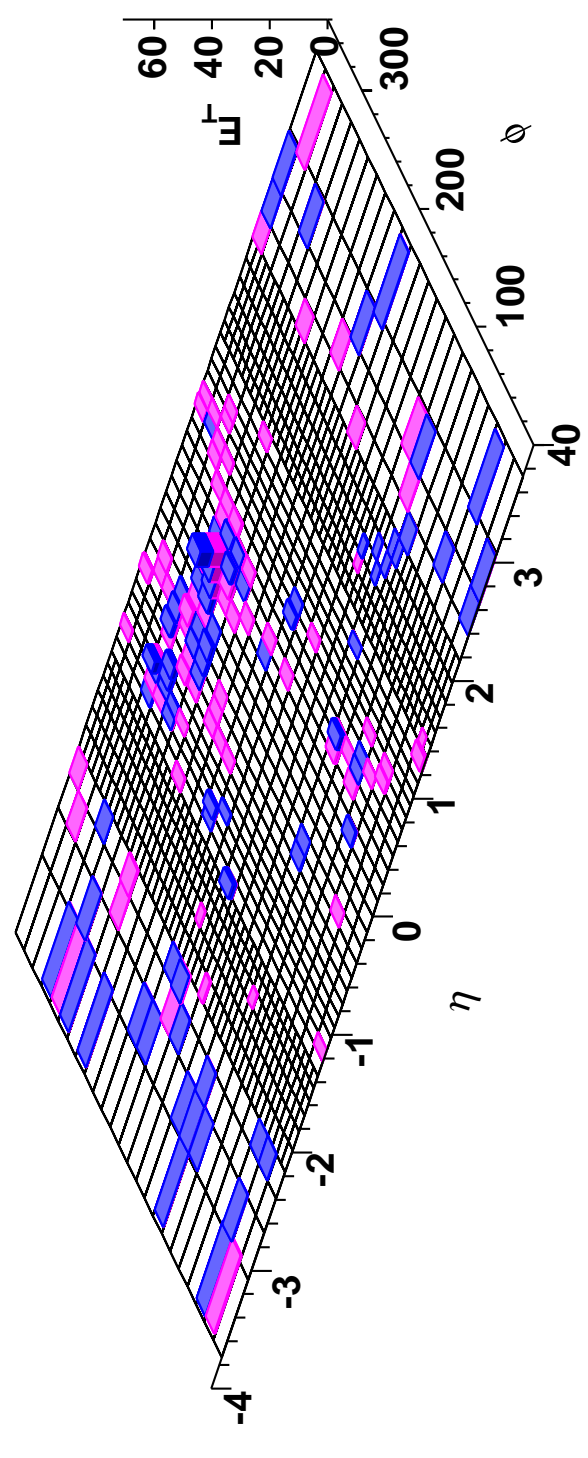

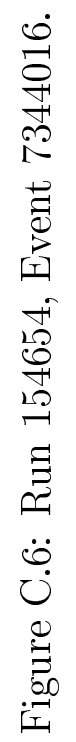




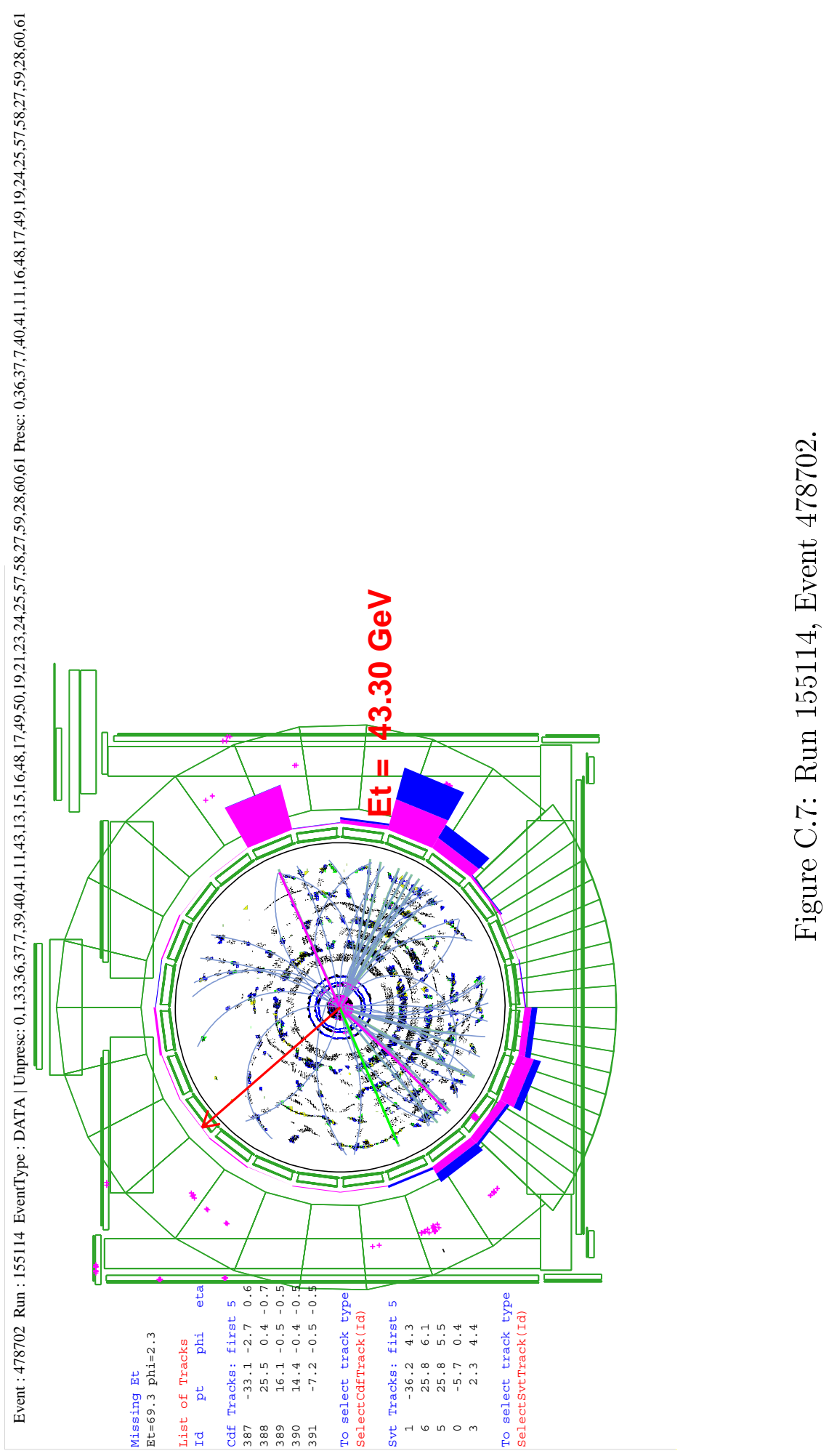




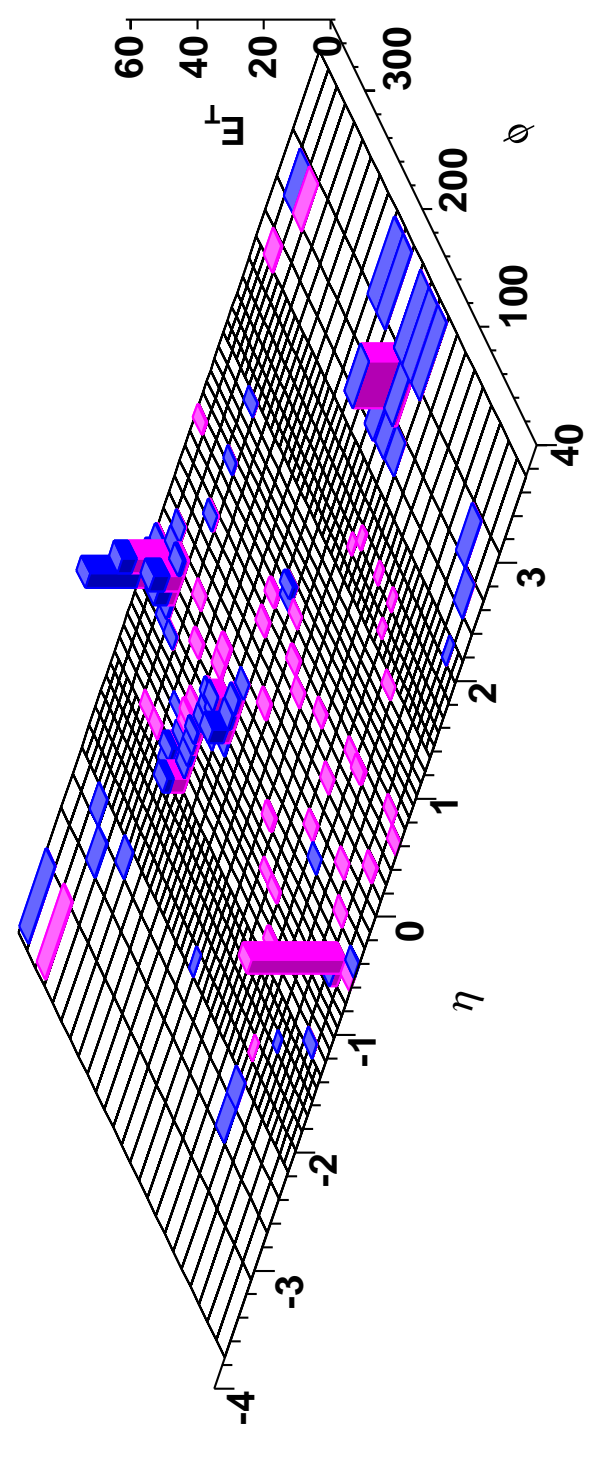

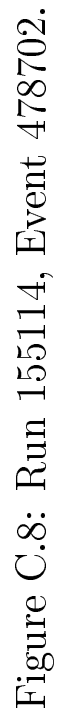




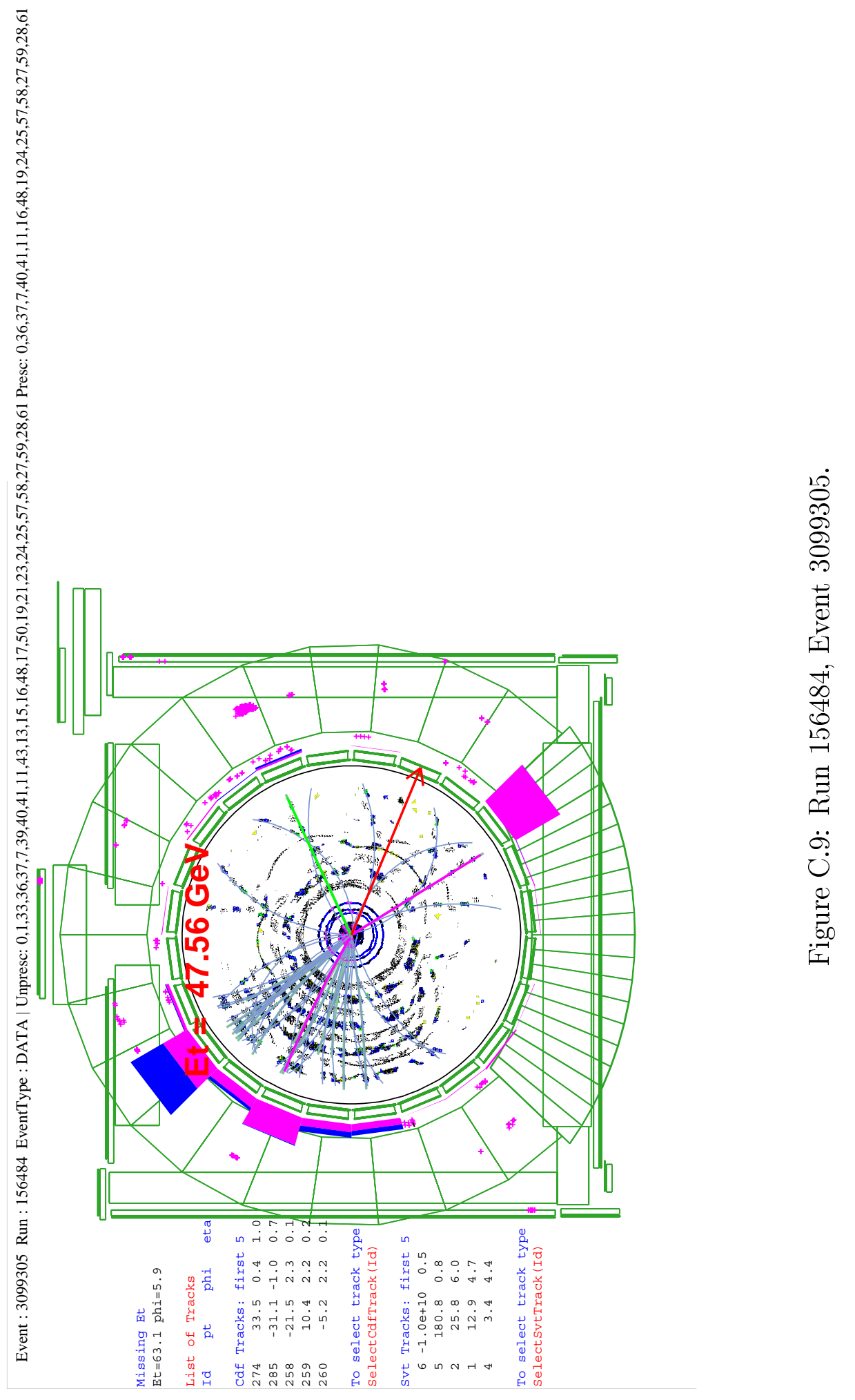




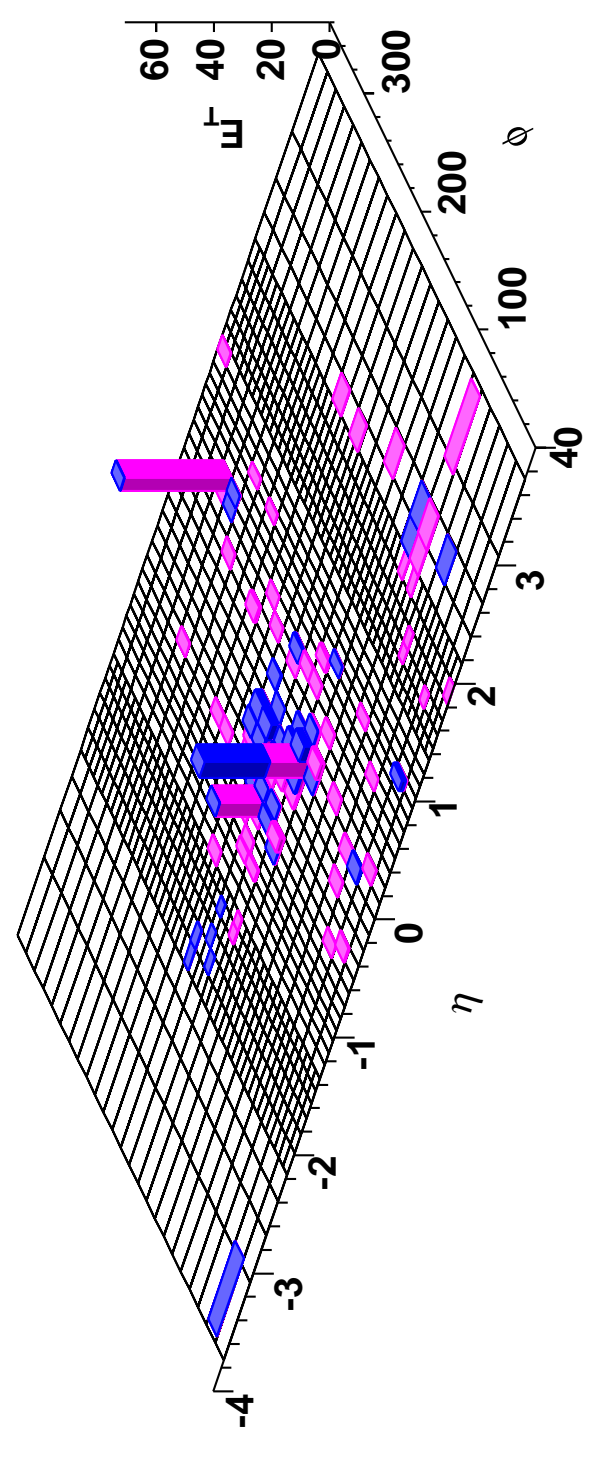

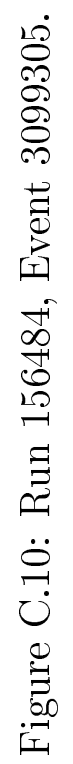




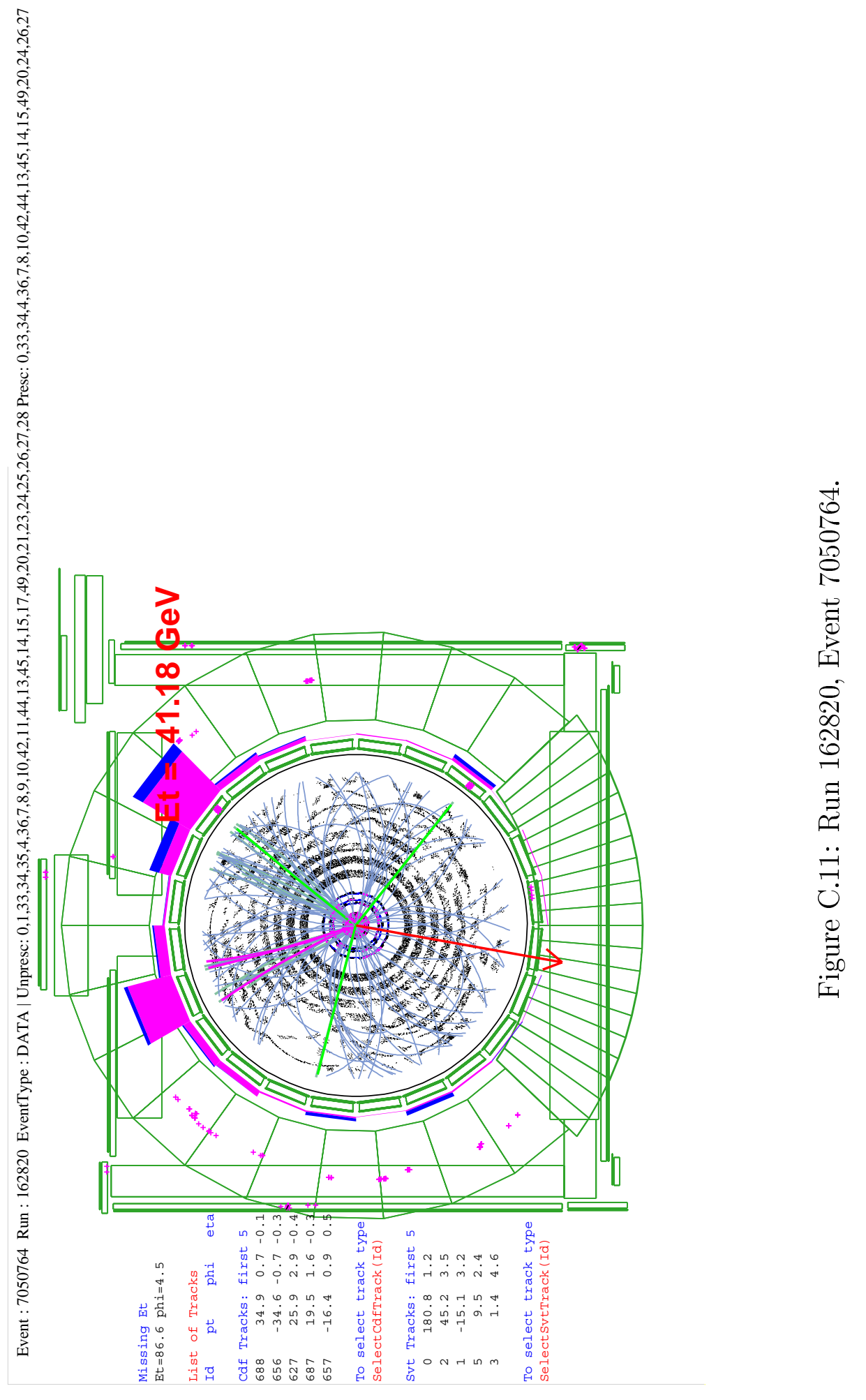




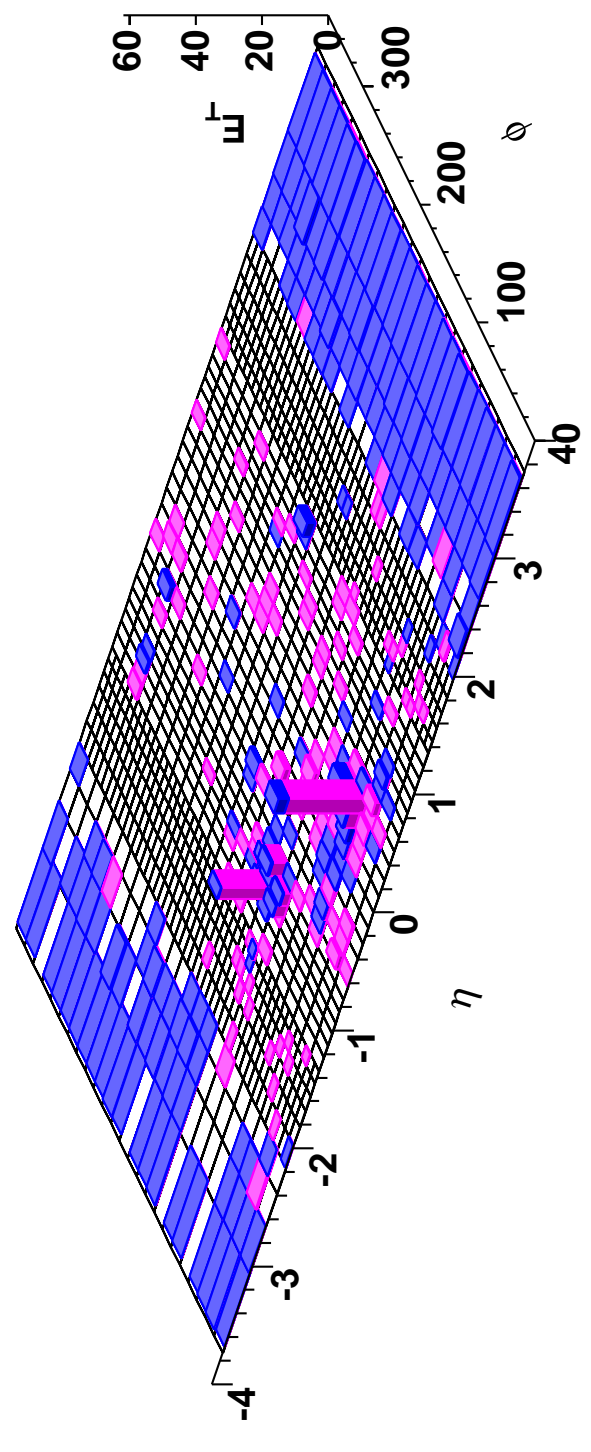

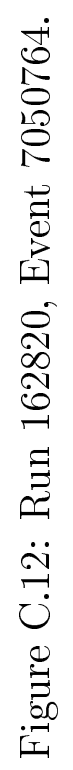




\section{BIBLIOGRAPHY}

[1] S. Glashow, Nucl. Phys. 22, 579 (1961); S. Weinberg, Phys. Rev. Lett. 19, 1264 (1967); A. Salam, in Elementary Particle Theory: Relativistic Groups and Analyticity (Nobel Symposium No. 8), edited by N. Svartholm (Almqvist and Wiksell, Stockholm, 1968), p.367.

[2] S. Glashow, J. Iliopoulos, and L. Maina, Weak Interactions with LeptonHadron Symmetry, Phys. Rev. D 2, 1285, (1970).

[3] N. Cabibbo, Phys. Rev. Lett. 10, 531 (1963).

[4] M. Kobayashi and T. Maskawa, Prog. Theor. Phys. 49, 652 (1973).

[5] K. Hagiwara et al., Phys. Rev. D 66, 010001 (2002).

[6] W. Bartel et al., A measurement of the electroweak induced charge asymmetry in $e^{+} e^{-} \rightarrow b \bar{b}$. Phys. Lett. B,146, 437 (1984).

[7] C. Itzykson, J.-B. Zuber, Quantum Field Theory, (McGraw-Hill, New York, 1980).

[8] W. Greiner, B. Muller, Gauge theory of weak interactions, (Springer-Verlag, Berlin, Heidelberg, 1996).

[9] C. Albajar et al., Z. Phys. C 48, 1 (1990).

[10] T. Akesson et al., Z. Phys. C 46, 179 (1990).

[11] F. Abe et al., Phys. Rev. Lett. 68, 447 (1992); F. Abe et al., Phys. Rev. D 45, 3921 (1992).

[12] S. Abashi et al., Phys. Rev. Lett. 72, 2138 (1994).

[13] F. Abe et al., (CDF Collaboration), Observation of top quark production in $p \bar{p}$ collisions, Phys. Rev. Lett. 74, 2626 (1995). 
[14] S. Abachi et al., (DØ Collaboration), Observation of the top quark, Phys. Rev. Lett. 74, 2632 (1995).

[15] T. Stelzer, Z. Sullivan, and S. Willenbrock, Phys. Rev. D 56, 5919 (1997).

[16] M. Cacciari et al., The $t \bar{t}$ Cross-section at 1.8 and $1.96 \mathrm{TeV}$ : A Study of the Systematics due to Parton Densities and Scale Dependence, CERN-TH/2003054, hep-ph/0303085, (2003).

[17] M. Hohlmann, Ph.D. dissertation, University of Chicago, August 1997.

[18] F. Abe et al., (CDF Collaboration), Measurement of the $t \bar{t}$ production cross section in $p \bar{p}$ collisions at $\sqrt{s}=1.8 \mathrm{TeV}$, Phys. Rev. Lett. 80, 2773 (1998).

[19] F. Abe et al., (CDF Collaboration), Measurement of the top quark mass and $t \bar{t}$ production cross section from dilepton events at the Collider Detector at Fermilab, Phys. Rev. Lett. 80, 2779 (1998).

[20] F. Abe et al., (CDF Collaboration), First observation of the all hadronic decay of $t \bar{t}$ pairs, Phys. Rev. Lett. 79, 1992 (1997).

[21] S. Abachi et al., (DØ Collaboration), Measurement of the top quark pair production cross section in $p \bar{p}$ collisions, Phys. Rev. Lett. 79, 1203 (1997).

[22] I. Bigi et al., Production and decay properties of ultra-heavy quarks, Phys. Lett. B 181, 157 (1986).

[23] L. Demortier et al., FERMILAB-TM-2084, (1999).

[24] K. Kodama et al., (DONUT Collaboration), Observation of tau neutrino interactions, Phys. Lett. B 504, 218 (2001).

[25] F. Abe et al., (CDF Collaboration), Evidence for top quark production in $p \bar{p}$ collisions at $\sqrt{s}=1.8 \mathrm{TeV}$, Phys. Rev. Lett. 73, 225 (1994).

[26] Fermilab Beams Division Run II Handbook, http://www-bd.fnal.gov/lug/runII_handbook/RunII_index.html.

[27] CDF Operation Manager Homepage (internal), http://www-cdfonline.fnal.gov/opshelp/stores/.

[28] The CDF II Detector Technical Design Report, FERMILAB-Pub-96/390-E.

[29] E. J. Thomson et al., Online Track Processor For The CDF Upgrade, IEEE Trans. Nucl. Sci. 49, 1063 (2002). 
[30] T. Nelson, et al., Forward Electron Tracking with the PhoenixMods Package, CDF/DOC/TRACKING/PUBLIC/6278, (2003).

[31] D. Goldstein, A measurement of the $W$ boson cross-section using electrons in the upgrade endplug detectors, CDF/PHYS/CDF/CDFR/5975, (2002).

[32] Anyes Taffard, Run II Cosmic Ray Tagger, CDF/PUB/MUON/PUBLIC/6100, (2003).

[33] Angela Wyatt, Beate Heinemann, Correction for Leakage Energy and Multiple Interactions for Electrions and Photons in the Central and Plug Calorimeters, CDF/ANAL/ELECTRON/CDFR/6167, (2002).

[34] L. Galtieri et al., How well do we understand jets in Run I? Study of the Jet Energy Scale for Raw Jet $E_{T}>8 \mathrm{GeV}$, CDF/ANAL/TOP/CDFR/3983, (1997).

[35] J.-F. Arguin et al., Systematic Uncertainties Associated with Jet Corrections for Winter 2003 Conferences, CDF/PHYS/JET/CDFR/6419, (2003).

[36] M. Coca, et al., Optimization of the Drell-Yan Background Removal for the Top Dilepton Selection, CDF/DOC/TOP/CDFR/6579, (2003).

[37] D. Goldstein, et al., A measurement of the $t \bar{t}$ cross-section using dileptons in the central and endplug detectors, CDF/PHYS/TOP/CDFR/6588, (2003).

[38] Torbjörn Sjöstrand et al., PYTHIA 6.2 Physics and Manual, hep-ph/0108264, (2001).

[39] H. Lai et al., Global QCD Analysis of Parton Structure of the Nucleon: CTEQ5 Parton Distributions, HEP-ph/9903282, (1999).

[40] G. Corcella et al., HERWIG 6.4 Release Note, hep-ph/0201201, (2001).

[41] G. Corcella et al., HERWIG 6.5: and event generator for Hadron Emission Reactions With Interfering Gluons, hep-ph/0011363, (2002).

[42] The information about the Monte Carlo samples with various structure functions used to estimate the systematic uncertainty can be found in the following web pages. http://www-cdf.fnal.gov/internal/physics/top/run2mass/PDFs/, http://www-cdf.fnal.gov/internal/physics/top/mc/ttbar_systematic.shtml.

[43] The HERWIG 6.4 does not support the QED final state radiation. However, the updated version of the HERWIG 6.504 supports this feature. The current version of the CDF offline software uses the HERWIG 6.4. It will be implemented in the future CDF offline software. 
[44] The effect of the final state gluon radiation was not studied in this thesis. This effect can by studied with a subset of no-ISR events where all jets are matched to partons from the hard scatter. These events presumably do not have hard gluon emissions.

[45] M. Kruse, Ph.D. dissertation, Purdue University, May 1996.

[46] S. Drell adn T.-M. Yan, Massive Lepton-Pair Production in Hadron-Hadron Collisions at High Energies, Phys. Rev. Lett. 25, 316 (1970).

[47] M. Contreras and J. Wang, Estimate of the Drell-Yan background in the Top Dilepton Analysis, CDF/ANAL/TOP/CDFR/2261, (1993).

[48] J. Konigsberg, Drell-Yan Backgrounds for the Top Dilepton Analysis in 100 $p b^{-1}, \mathrm{CDF} / \mathrm{DOC} / \mathrm{TOP} / \mathrm{CDFR} / 3208,(1995)$.

[49] F. Berends et al., Nucl. Phys., B357, 32, (1991).

[50] M. Kruse et al., High $p_{T}$ Lepton ID Efficiencies for Run 1b, CDF/DOC/TOP/CDFR/2883, (1994).

[51] J. Campbell, R. Ellis, An update on vector boson pair production at hadron colliders, hep-ph/9905386, (1999).

[52] $W W$ Production Cross Section in the Dilepton Channel at CDF in Run II, S. Cabrera et al., CDF/ANAL/ELECTROWEAK/GROUP/6323, (2003).

[53] CDF Top Group Data Samples web page, http://www-cdf.fnal.gov/internal/physics/top/topdata/newtopdata.html.

[54] F. James, MINUIT, Reference Manual, Version 94.1, CERN Program Library Long Writeup D506, (1998). 